Aus dem Forschungs- und Studienzentrum für Veredelungswirtschaft

Weser-Ems der Georg-August-Universität Göttingen

Fakultät für Agrarwissenschaften

\title{
Verhalten von ausgewählten Tetrazyklinen und Sulfonamiden in Wirtschaftsdünger und in Böden
}

\author{
Dissertation \\ zur Erlangung des Doktorgrades \\ der Fakultät für Agrarwissenschaften \\ der Georg-August-Universität Göttingen
}

vorgelegt von

Heike Engels

geboren in Bremen

Göttingen, 2004 
1. Referent: Prof. Dr. Ir. H. Van den Weghe

2. Korreferent: Prof. Dr. C. Winckler

Woche der mündlichen Prüfungen: 05.07.-09.07.2004 


\section{Inhaltsverzeichnis}

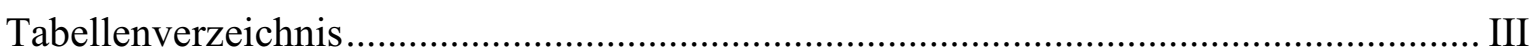

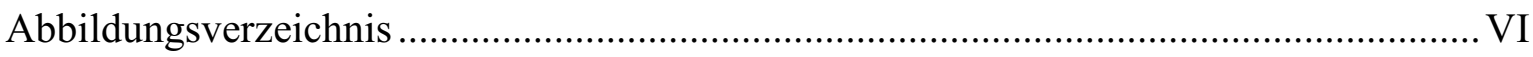

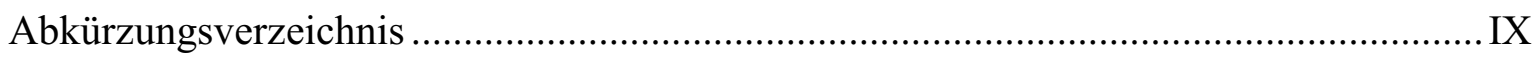

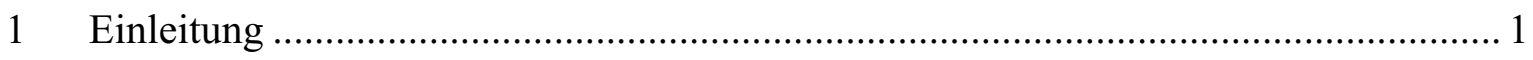

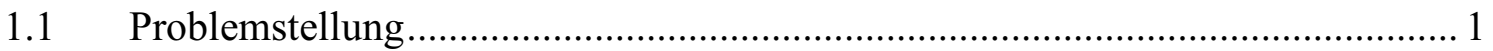

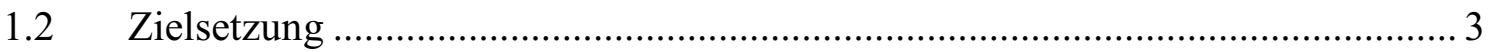

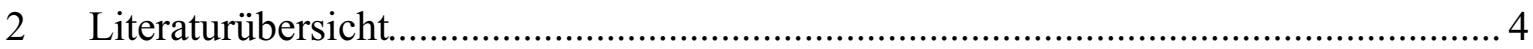

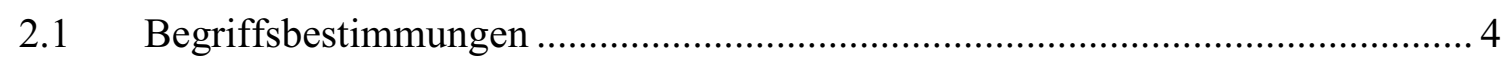

2.2 Physikochemische und pharmakokinetische Eigenschaften ausgewählter

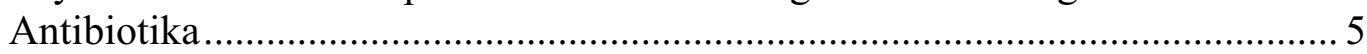

2.3 Persistenz von Tierarzneimitteln in verschiedenen Medien............................... 10

2.4 Vorkommen und Mobilität von Tierarzneimitteln in Böden............................... 15

2.5 Vorkommen von Antibiotika in Pflanzen......................................................... 19

3 Material und Methoden ...................................................................................... 21

3.1 Entwicklung von Nachweismethoden .......................................................... 21

3.1.1 Tetrazyklin- $\mathrm{HCl}$ in Masthähnchenfestmist................................................. 22

3.1.2 Ausgewählte Tetrazykline und Sulfonamide in Schweinegülle................... 24

3.1.3 Tetrazyklin und Sulfadiazin in Sickerwasser............................................ 30

3.2 Persistenz von Tetrazyklin in Masthähnchenfestmist ......................................... 35

3.2.1 Versuchsaufbau und Vorgehensweise von Experiment 1........................... 36

3.2.2 Versuchsaufbau und Vorgehensweise von Experiment 2........................ 38

3.3 Screening von Schweinegülleproben auf ausgewählte Antibiotika.................... 39

3.4 Mobilität von Tetrazyklin und Sulfadiazin in Böden........................................ 40

3.4.1 Versuchsaufbau und Vorgehensweise Kick-Brauckmann-Gefäße.............. 40

3.4.2 Versuchsaufbau und Vorgehensweise Lysimeter....................................... 43

3.5 Weitere Analysemethoden und statistische Auswertung ................................. 45

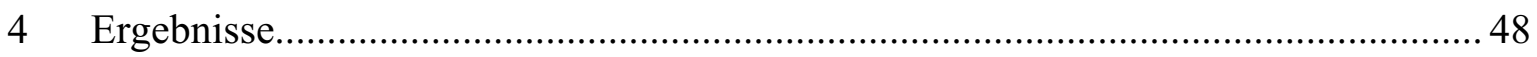

4.1 Modellversuche zur Persistenz von Tetrazyklin in Masthähnchenfestmist........ 48

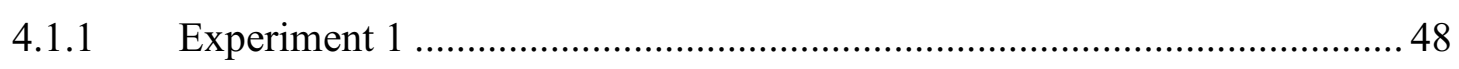

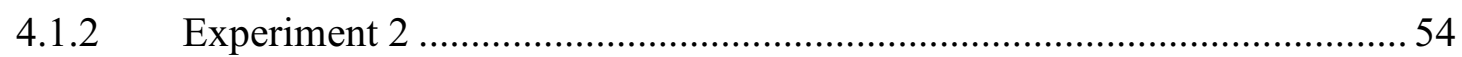

4.2 Nachweis ausgewählter Tetrazykline und Sulfonamide in Schweinegülle......... 59

4.2.1 Screening anonymer Schweinegülleproben 1999/2000 _........................... 59

4.2.2 Screening anonymer Schweinegülleproben 2001/2002 _........................... 62

4.3 Modellversuche zur Mobilität von Tetrazyklin und Sulfadiazin in Böden......... 66 


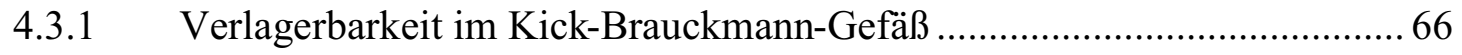

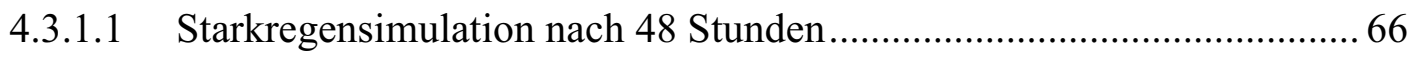

4.3.1.2 Starkregensimulation nach 10 Wochen ................................................... 70

4.3.2 Verlagerbarkeit im bewirtschafteten Großlysimeter................................. 72

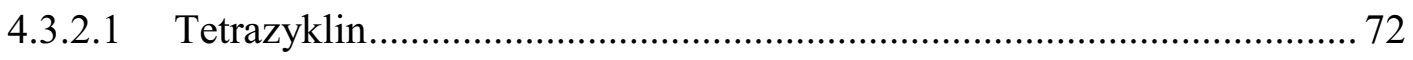

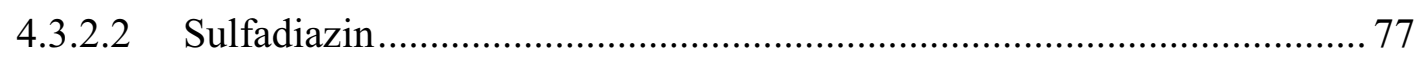

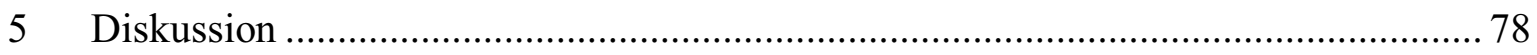

5.1 Modellversuche zur Persistenz von Tetrazyklin in Masthähnchenfestmist........ 78

5.2 Nachweis ausgewählter Tetrazykline und Sulfonamide in Schweinegülle.......... 84

5.3 Modellversuche zur Mobilität von Tetrazyklin und Sulfadiazin in Boden.......... 88

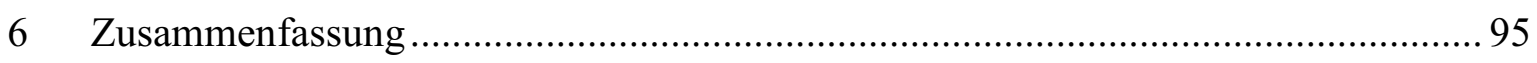

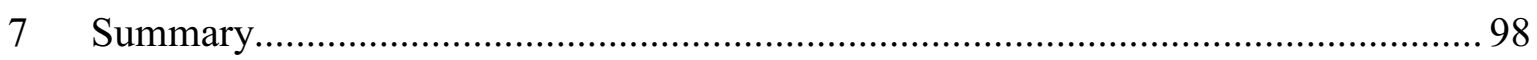

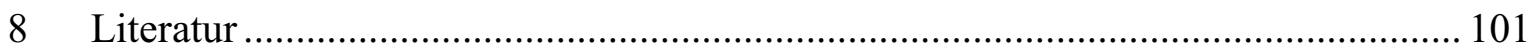

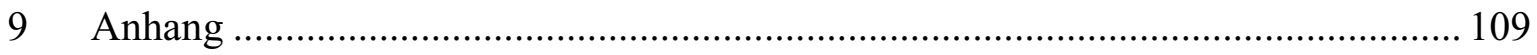




\section{Tabellenverzeichnis}

Tabelle 1: Pharmakokinetische Daten von Tetrazyklinen (Kroker et al. 1996)............ 7

Tabelle 2: $\quad$ Umweltrelevante physiko-chemische Eigenschaften wie Löslichkeit, Volatilität, Säurekonstante $\left(\mathrm{pK}_{\mathrm{s}}\right)$ und Oktanol-Wasser-

Verteilungskoeffizient $\left(\log \mathrm{K}_{\mathrm{ow}}\right)$ ausgewählter Veterinärantibiotika (Römpp 1995; Thiele-Bruhn 2003a).

Tabelle 3: $\quad$ Adsorptionskoeffizienten an die Bodenmatrix $\left(K_{\mathrm{f}}\right)$, an die organische Substanz im Boden $\left(K_{\mathrm{oc}}\right)$ sowie an die Tonfraktion im Boden $\left(K_{\text {Ton }}\right)$ ausgewählter pharmazeutischer Antibiotika in Böden und Wirtschaftsdüngern $\left(K_{\mathrm{oc}}\right.$-Werte sind normiert auf den organischen Kohlenstoffgehalt) (Tolls 2001; Thiele-Bruhn 2003b)

Tabelle 4: $\quad$ Gradientenprogramm für den Tetrazyklinnachweis in Masthähnchenfestmist.

Tabelle 5: Wiederfindungsrate (\%) von Tetrazyklin (TC) einschließlich 4Epi-

Tetrazyklin in Masthähnchenfestmist (Frischmist)

Tabelle 6: Gradientenprogramm für den Tetrazyklin- und Sulfonamidnachweis in Schweinegülle

Tabelle 7: $\quad$ Ergebnisse der Varianzanalyse mit den Signifikanzniveaus und Freiheitsgraden (df) innerhalb der Konzentrationsstufen (Konz I- IV) der untersuchten Antibiotika in Schweinegülle (Modell 1)....

Tabelle 8: $\quad$ Ergebnisse der Varianzanalyse mit den Signifikanzniveaus und Freiheitsgraden (df) innerhalb der untersuchten Antibiotika Tetrazyklin (TC), Oxytetrazyklin (OTC), Chlortetrazyklin (CTC), Sulfadiazin (SD) und Sulfamethazin (SM) in Schweinegülle ( $\mathrm{G}=$ Gülle, $\mathrm{d}=$ Tag, $\mathrm{K}=$ Konzentration) (Modell 2).

Tabelle 9: Wiederfindungsraten (\%) für ausgewählte Antibiotikasubstanzen in Schweinegülle.....

Tabelle 10: Gradientenprogramm für den Tetrazyklinnachweis in Sickerwasser bei einer Laufzeit von $15 \mathrm{~min}$

Tabelle 11: Gradientenprogramm für den Sulfadiazinnachweis in Sickerwasser bei einer Laufzeit von $17 \mathrm{~min}$

Tabelle 12: $\quad$ Wiederfindungsraten (\%) für Tetrazyklin (TC) und Chlortetrazyklin (CTC) in Sickerwasser bei Kalibration mit internem Standard

Tabelle 13: $\quad$ Wiederfindungsrate (\%) von Sulfadiazin in Sickerwasser bei

Kalibration mit internem Standard.

Tabelle 14: Übersicht zu Versuchsanordnung und-bedingungen Experiment 1

$(\mathrm{V}=$ Variante, $\mathrm{TC}=$ Tetrazyklin $)$

Tabelle 15: $\quad$ Übersicht zu Versuchsanordnung und-bedingungen Experiment 2 $(\mathrm{V}=$ Variante $)$ 39

Tabelle 16: Kenndaten der verwendeten Böden im Gefäßversuch.

Tabelle 17: Kenndaten der in den Lysimetern verwendeten Böden, aufgeteilt nach Oberboden (Krume) und Unterboden. 
Tabelle 18: Übersicht zur Versuchsanordnung bei der Untersuchung der Mobilität von Tetrazyklin und Sulfadiazin im Lysimeter $(\mathrm{TC}=$ Tetrazyklin; $\mathrm{SD}=$ Sulfadiazin; $\mathrm{N}=$ Stickstoff) $)$.

Tabelle 19: $\quad$ Stoffkenngrößen wie Stickstoff- (N), Kohlenstoff- (C), organischer Substanzgehalt (OS) des im Experiment 1 eingesetzten Masthähnchenfestmistes zu Beginn der Lagerung (in \% bezogen auf den TS-Gehalt).... 48

Tabelle 20: $\quad$ Stoffkenngrößen wie Stickstoff- $(N)$, Kohlenstoff- $(C)$, organischer Substanzgehalt (OS) des im Experiment 1 eingesetzten Masthähnchenfestmistes am Ende der Lagerung (in \% bezogen auf den TS-Gehalt)

Tabelle 21: $\quad$ Stoffkenngrößen wie Stickstoff- (N), Kohlenstoff- (C), organischer Substanzgehalt (OS) des im Experiment 2 eingesetzten Masthähnchenfestmistes zu Beginn der Lagerung (in \% bezogen auf den TS-Gehalt).... 55

Tabelle 22: $\quad$ Stoffkenngrößen wie Stickstoff- (N), Kohlenstoff- (C), organischer Substanzgehalt (OS) des im Experiment 2 eingesetzten Masthähnchenfestmistes am Ende der Lagerung (in \% bezogen auf den TS-Gehalt) ...... 55

Tabelle 23: $\quad$ Trockensubstanz- (TS) und Stickstoffgehalte (N) der Schweinegülleproben 1999/2000.

Tabelle 24: $\quad$ Ergebnisse des Screenings von Schweinegülleproben 1999/2000 60

Tabelle 25: Verteilung der Schweinegülleproben und der nachgewiesenen Antibiotika in vier Trockensubstanzklassen (TS), Screening 1999/2000 (Median der positiven Proben).

Tabelle 26: $\quad$ Trockensubstanz- (TS) und Stickstoffgehalte (N) der Schweinegülleproben 2001/2002.

Tabelle 27: Ergebnisse des Screenings von Schweinegülleproben 2001/2002

Tabelle 28: Verteilung der Schweinegülleproben und der nachgewiesenen Antibiotika in vier Trockensubstanzklassen (TS), Screening 2001/2002 (Median der positiven Proben).

Tabelle 29: $\quad$ Signifikante Effekte zwischen den einzelnen Trockensubstanz (TS)Klassen der Schweinegülleproben 2001/2002 bezüglich verschiedener Antibiotika (Mann-Whitney-U-Test)....

Tabelle 30: Gehalte an Tetrazyklin (TC) und Sulfadiazin (SD) $\left(\mu \mathrm{g} 1^{-1}\right)$ im Sickerwasser (je Fraktion $100 \mathrm{ml}$ ) bei Beregnung 48 Stunden nach Gülleaufbringung

Tabelle 31: Tetrazyklin (TC)-Gehalte $\left(\mu \mathrm{g}^{-1}\right)$ im Sickerwasser unter Mais in den ersten vier Wochen nach Gülleausbringung, Durchgang 2001 (1 = humoser Sand I; 2 = humoser Sand II).

Tabelle 32: Gegenüberstellung der Medianwerte unter Berücksichtigung der positiv getesteten Proben bzw. aller Proben der beiden SchweinegülleProbensätze

Tabelle 33: $\quad$ PEC (Predicted Environmental Concentration) für ausgewählte Wirkstoffe in Boden auf Basis der Untersuchungsergebnisse aus Screening 1 und 2 (Median der positiven Proben) bei verschiedenen Einarbeitungstiefen (ET). 
Tabelle 34: Tagesmittelwerte des Temperaturverlaufs im Masthähnchenfestmist der sechs Komposter und der Raumluft, Experiment 1.

Tabelle 35: Tagesmittelwerte des Temperaturverlaufs im Masthähnchenfestmist der acht Komposter und der Raumluft, Experiment 2.

Tabelle 36: Tetrazyklin-Gehalte im Masthähnchenfestmist der sechs Komposter über den dreimonatigen Lagerungszeitraum, Experiment 1.

Tabelle 37: Tetrazyklin-Gehalte im Masthähnchenfestmist der acht Komposter über den zweimonatigen Lagerungszeitraum, Experiment 2.

Tabelle 38: Ermittelte Wirkstoffkonzentrationen von Tetrazyklin (TC), Oxytetrazyklin (OTC), Chlortetrazyklin (CTC), Sulfadiazin (SD) und Sulfamethazin (SM) des Screenings der Schweinegülleproben aus dem Jahr 1999/2000, $n=168$ (TS = Trockensubstanz; N = Stickstoff).. 113

Tabelle 39: $\quad$ Ermittelte Wirkstoffkonzentrationen von Tetrazyklin (TC), Oxytetrazyklin (OTC), Chlortetrazyklin (CTC), Sulfadiazin (SD) und Sulfamethazin (SM) des Screenings der Schweinegülleproben aus dem Jahr 2001/2002, n = 176 (TS = Trockensubstanz; N = Stickstoff).. 117

Tabelle 40: Vergleich der mittels HPLC gemessenen Tetrazyklin-Konzentrationen im Sickerwasser unter Nullerde/Torf des Gefäßversuchs (Starkregenereignis 48 Stunden nach Gülleausbringung) mit den Ergebnissen der Referenzmessung (siehe Kapitel 3.4.1).

Tabelle 41: Tetrazyklin-Gehalte im Boden der Kick-Brauckmann-Gefäße, Starkregenereignis 48 Stunden bzw. zehn Wochen nach Gülleausbringung.....

Tabelle 42: $\quad$ Sulfadiazin-Gehalte im Boden der Kick-Brauckmann-Gefäße, Starkregenereignis 48 Stunden bzw. 10 Wochen nach Gülleausbringung....

Tabelle 43: Vergleich der mittels HPLC gemessenen Tetrazyklin-Konzentrationen im Sickerwasser des Lysimeterversuches mit den Werten der Referenzmessung (siehe Kapitel 3.4.2).

Tabelle 44: Verlauf der Tetrazyklin-Gehalte (TC) im Boden ausgewählter Lysimeter während der Versuchsdauer (TC-Gabe über Gülle jeweils im Mai 2001 (Roggen früher, Mais später) und Mai 2002; Dosierung je Lysimeter: Lys. 4 und $28=45,0 \mathrm{mg}$ bzw. 44,3 mg TC; Lys. 13, 15, 33, $35=67,2$ bzw. 66,5 mg TC).

Tabelle 45: Verlauf der Sulfadiazin-Gehalte (SD) im Boden ausgewählter Lysimeter während der Versuchsdauer (SD-Gabe über Gülle im Mai 2002; Dosierung je Lysimeter: Lys. 4 und $28=44,3 \mathrm{mg} \mathrm{SD}$; Lys. 13, 15, 33, $35=66,5 \mathrm{mg} \mathrm{SD})$.

Tabelle 46: Verlauf der Chlortetrazyklin-Gehalte im Boden ausgewählter Lysimeter während der Versuchsdauer (Nebenbefund). 


\section{Abbildungsverzeichnis}

Abbildung 1: Eintragspfade für Antibiotika in die Umwelt........................................... 1

Abbildung 2: $\quad$ Struktur der Tetrazykline $(\mathrm{R}=\mathrm{Rest})($ Kroker et al. 1996)........................... 6

Abbildung 3: Struktur ausgewählter Sulfonamide (Haller et al. 2002)............................ 8

Abbildung 4: Verteilung ausgewählter Antibiotika (Tetrazyklin (TC), Chlortetrazyklin (CTC), Sulfadiazin (SD), Sulfamethazin (SM)) in der Fest-, Misch- und Flüssigphase einer Schweinegülle (mit Standardabweichung T, Mittelwert aus vier Wiederholungen).

Abbildung 5: Wiederfindungsrate (\%) für Tetrazyklin und Chlortetrazyklin bei ausgewählten Extraktionsmitteln (Oxalsäure = methanolische Oxalsäure $0,01 \mathrm{M}$; Trichloressigs. $=0,1 \%$ Trichloressigsäure in Methanol) bei $\mathrm{pH}$ 4,8 (externe Kalibration, Mittelwert aus drei Messungen).

Abbildung 6: Wiederfindungsrate (\%) für Tetrazyklin und 4-Epi-Tetrazyklin (Epi-TC) + Tetrazyklin (TC) mit verschiedenen Konzentrationen des Extraktionsmittels Trichloressigsäure in Methanol (TCSM) bei pH 4,8 (externe Kalibration, Mittelwert aus drei Messungen).

Abbildung 7: Kompostbehälter und durchmischter Masthähnchenfestmist beim Umsetzen der locker geschichteten Varianten.

Abbildung 8: Schematische Darstellung der Versuchsanordnung beim

Lagerungsversuch $(\mathrm{RM}=$ Rottemist, $\mathrm{SM}=$ Stapelmist, $\mathrm{V}=$ Variante, $\mathrm{TC}=$ Tetrazyklin).

Abbildung 9: Homogenisierung, Befüllen der Komposter und Dotierung des Masthähnchenfestmistes mit Tetrazyklin.

Abbildung 10: Kick-Brauckmann-Gefäße und Beprobungsvorrichtung zum Auffangen der Sickerwasserfraktionen.

Abbildung 11: Mit Winterroggen bestellte Lysimeter und Lysimetergang mit Sickerwasserauffangbehältern .....

Abbildung 12: Mittlerer Temperaturverlauf $\left({ }^{\circ} \mathrm{C}\right)$ des Substrats für die Lagerungsvarianten Rottemist und Stapelmist während der dreimonatigen Lagerung, Experiment 1.

Abbildung 13: Mittlerer Temperaturverlauf $\left({ }^{\circ} \mathrm{C}\right)$ des Substrats in den Varianten mit unterschiedlicher Tetrazyklin (TC)-Konzentration während der dreimonatigen Lagerung, Experiment 1

Abbildung 14: Verlauf der Konzentration an Tetrazyklin (TC) $\left(\mathrm{mg} \mathrm{kg}^{-1}\right)$ im Masthähnchenfestmist (Rotte (RM)- bzw. Stapelmist (SM)) der Varianten $0,5 \mathrm{~g} \mathrm{TC} \mathrm{kg}^{-1}$ Futter und $1,0 \mathrm{~g} \mathrm{TC} \mathrm{kg}^{-1}$ Futter über die dreimonatige Lagerung, bezogen auf Trockensubstanz (\%)..

Abbildung 15: Verlauf der Konzentration an Tetrazyklin (TC) $\left(\mathrm{mg} \mathrm{kg}^{-1}\right)$ im getrockneten Masthähnchenfestmist der Varianten $0,5 \mathrm{~g} \mathrm{TC} \mathrm{kg}^{-1}$ Futter und 1,0 $\mathrm{g} \mathrm{TC} \mathrm{kg}^{-1}$ Futter (Rotte (RM)- bzw. Stapelmist (SM)) über die dreimonatige Lagerung, bezogen auf Kohlenstoff...

Abbildung 16: Verlauf der relativen Tetrazyklin (TC)-Konzentration (\%) bei Rotte (RM)- bzw. Stapelmist (SM) über die dreimonatige Lagerung, bezogen auf die Trockensubstanz (\%). 
Abbildung 17: Mittlerer Temperaturverlauf $\left({ }^{\circ} \mathrm{C}\right)$ des Substrats für die

Lagerungsvarianten Rottemist und Stapelmist während der

zweimonatigen Lagerung, Experiment 2

Abbildung 18: Mittlerer Temperaturverlauf $\left({ }^{\circ} \mathrm{C}\right)$ des Substrates der Rotte- und Stapelmistvarianten mit Tetrazyklin (TC)-Konzentration während der zweimonatigen Lagerung, Experiment 2 .....

Abbildung 19: Verlauf der relativen Konzentration an Tetrazyklin (TC) (\%) (mit Standardabweichung $T$, Mittelwert aus drei Messwerten) in den Varianten Rotte- und Stapelmist über die zweimonatige Lagerung, bezogen auf Trockensubstanz (\%).

Abbildung 20: Verlauf der relativen Konzentration (mit Standardabweichung , Mittelwert aus drei Messwerten) an Tetrazyklin (TC) (\%) in den Varianten Rotte- und Stapelmist (bezogen auf den Trockensubstanzgehalt) über die zweimonatige Lagerung, bezogen auf Kohlenstoff.

Abbildung 21: Ergebnisse des Screenings 1999/2000 für vier ausgewählte Antibiotika in Schweinegülleproben $(\mathrm{n}=168)$. Box-Whisker-Plot zeigt Median (Querstrich), $10 \%, 25 \%, 75 \%$ und $90 \%$ Perzentil mit Fehlerbalken. Die Punkte stellen die Einzelwerte außerhalb des $10 \%$ und 90 \% Perzentils dar. Aus Gründen der Übersichtlichkeit sind zwei Ausreißerwerte für Chlortetrazyklin $\left(330,7 \mathrm{mg} \mathrm{kg}^{-1}\right)$ bzw. Sulfadiazin $\left(235,1 \mathrm{mg} \mathrm{kg}^{-1}\right)$ nicht dargestellt.

Abbildung 22: Ergebnisse des Screenings 2001/2002 für die Antibiotika Tetrazyklin (TC), Oxytetrazyklin (OTC), Chlortetrazyklin (CTC), Sulfadiazin (SD) und Sulfamethazin (SM) in Schweinegülleproben $(\mathrm{n}=176)$. Box-Whisker-Plot zeigt Median (Querstrich), $10 \%, 25 \%, 75 \%$ und $90 \%$ Perzentil mit Fehlerbalken. Die Punkte stellen die Einzelwerte außerhalb des $10 \%$ und $90 \%$ Perzentils dar. Aus Gründen der Übersichtlichkeit sind zwei Ausreißerwerte für OTC $\left(136,2 \mathrm{mg} \mathrm{kg}^{-1}\right)$ bzw. SM $\left(167,0 \mathrm{mg} \mathrm{kg}^{-1}\right)$ nicht dargestellt..

Abbildung 23: Tetrazyklin (TC)-Konzentrationen in den Sickerwasserfraktionen HIV (je Fraktion $100 \mathrm{ml}$ ) der Variante Nullerde/Torf, Starkregenereignis 48 Stunden nach Gülleaufbringung (Gefäß 2 aufgrund Negativbefund nicht dargestellt).

Abbildung 24: Mittlere Tetrazyklin (TC)-Konzentration in den oberen 0-12 cm und den unteren 12-24 cm der Versuchsböden (hS = humoser Sand; $1 \mathrm{U}=$ lehmiger Schluff; $N=$ Nullerde) je Gefäß; Starkregenereignis 48 Stunden nach Gülleaufbringung (Gefäß $1 \mathrm{U} 2$ ist nicht dargestellt).

Abbildung 25: Mittlere Sulfadiazin (SD)-Konzentration in den oberen 0-12 cm der Versuchsböden (hS = humoser Sand; $1 \mathrm{U}=$ lehmiger Schluff; $\mathrm{N}=$ Nullerde) je Gefäß; Starkregenereignis 48 Stunden nach Gülleaufbringung.

Abbildung 26: Mittlere Tetrazyklin (TC)-Konzentration in den oberen 0-12 cm und den unteren 12-24 cm der Versuchsböden (hS = humoser Sand; $1 \mathrm{U}=$ lehmiger Schluff; N = Nullerde) je Gefäß; Starkregenereignis zehn Wochen nach Gülleaufbringung.... 
Abbildung 27: Mittlere Sulfadiazin (SD)-Konzentration in den oberen 0-12 cm der Versuchsböden (hS = humoser Sand; $1 \mathrm{U}=$ lehmiger Schluff; $\mathrm{N}=$ Nullerde) je Gefäß; Starkregenereignis zehn Wochen nach Gülleaufbringung.

Abbildung 28: Sickerwasseranfall unter Lysimeter 2 und 13 (humoser Sand 1), Mais, Durchgang 2001, Tetrazyklin (TC)-Befund: 19. Kalenderwoche (Lysimeter 2) und 22. Kalenderwoche (Lysimeter 13).

Abbildung 29: Sickerwasseranfall unter Lysimeter 58 und 65 (humoser Sand 2), Mais, Durchgang 2001, Tetrazyklin (TC)-Befund: 21. Kalenderwoche (Lysimeter 58 und Lysimeter 65).

Abbildung 30: Verlauf der Tetrazyklin (TC)-Konzentration im Boden bei ausgewählten Lysimetern, Fruchtart Roggen, Zeitraum Frühjahr 2001 bis Herbst 2002

Abbildung 31: Verlauf der Tetrazyklin (TC)-Konzentration im Boden bei ausgewählten Lysimetern, Fruchtart Mais, Zeitraum Herbst 2001 bis Herbst 2002, 1. TC-Applikation Mai 2001: Lys. 4 und 28: 45,0 mg; Lys. 13 und 35: 67,2 $\mathrm{mg}$ )

Abbildung 32: Verlauf der Sulfadiazin (SD)-Konzentration im Boden bei ausgewählten Lysimetern, Fruchtart Mais und Roggen, Zeitraum Frühjahr 2002 bis Herbst 2002 (Lys. 4 + 28: 44,3 mg SD; Lys. $13+35+15+33: 66,5 \mathrm{mg} \mathrm{SD})$

Abbildung 33: Halbwertszeiten (HWZ) von Tetrazyklin (TC) in Masthähnchenfestmist (Experiment 2) während der zweimonatigen Lagerung als Rotte (RM)bzw. Stapelmist (SM) in halblogarithmischer Darstellung (mittlere TC-Anfangskonzentration: Rottemist $=51,1 \mathrm{mg} \mathrm{kg}^{-1}$; Stapelmist $=43,6 \mathrm{mg} \mathrm{kg}^{-1}$ ) 


\section{Abkürzungsverzeichnis}

$\mu \mathrm{g}$

$\mu 1$

AAS

BGVV

${ }^{\circ} \mathrm{C}$

C

$\mathrm{CaCl}_{2}$

CTC

$\mathrm{CuO}$

CVMP

d

df

DüngeVO

EMEA

et al.

EU

EWG

FEDESA

$\mathrm{g}$

$\mathrm{h}$

ha

$\mathrm{HCl}$

HPLC

$\mathrm{K}$

$\mathrm{K}_{2} \mathrm{O}$

$K_{\mathrm{f}} / K_{\text {oc }} / K_{\text {Ton }}$

KAK

KOM

KT

KW

1

LC-ESI-MS

LC-MS-MS

$\log \mathrm{K}_{\mathrm{ow}}$

$\mathrm{M}$

$\mathrm{Mg}$

mval
Mikrogramm

Mikroliter

Atomabsorptionsspektrometer

Bundesinstitut für gesundheitlichen Verbraucherschutz und Veterinärmedizin

Grad Celsius

Kohlenstoff

Kalziumchlorid

Chlortetrazyklin

Kupferoxid

Commitee for Veterinary Medicinal Products

Tag

Freiheitsgrade

Dünge-Verordnung

European Agency for the Evaluation of Medicinal Products et alii (und andere)

Europäische Union

Europäische Wirtschaftsgemeinschaft

European Federation of Animal Health Industry

Erdbeschleunigung

Stunde

Hektar

Hydrochlorid

High Performance Liquid Chromatography

Kalium

Kaliumoxid

Bodenadsorptionskoeffizienten, bezogen auf Bodenmatrix, organische Substanz, Tongehalt

Kationenaustauschkapazität

Europäische Kommission

Kontrolle

Kalenderwoche

Liter

Liquid Chromatography Electrospray Mass Spectrometry

Liquid Chromatography Tandem Mass Spectrometry

n-Octanol-Wasser-Verteilungskoeffizient (logarithmiert)

Mol

Magnesium

Milliäquivalent, $10 \mathrm{mval} / \mathrm{kg}=1 \mathrm{cmol}_{\mathrm{c}} / \mathrm{kg}$ 


$\begin{array}{ll}\text { MW } & \text { Mittelwert } \\ \mathrm{n} & \text { Anzahl } \\ \mathrm{N} & \text { Stickstoff } \\ \mathrm{nm} / \mathrm{ng} & \text { Nanometer/ Nanogramm } \\ \varnothing & \text { Durchmesser, Durchschnitt } \\ \text { OS } & \text { Organische Substanz } \\ \text { OTC } & \text { Oxytetrazyklin } \\ \mathrm{Pa} & \text { Pascal } \\ \text { PEC } & \text { Predicted Environmental Concentration } \\ \mathrm{pK} & \text {-log (Säurekonstante) } \\ \mathrm{pH} & \text {-log (H' Konzentration) } \\ \mathrm{pt} & \text { Platin } \\ \mathrm{r}^{2} & \text { Bestimmtheitsmaß } \\ \text { SD } & \text { Sulfadiazin } \\ \text { SM } & \text { Sulfamethazin } \\ \text { SPSS } & \text { Statistical Products and Service Solutions } \\ \text { STD } & \text { Standardabweichung } \\ \text { SW } & \text { Sickerwasser } \\ \mathrm{t} & \text { Tonne } \\ \text { TC } & \text { Tetrazyklin } \\ \text { TS } & \text { Trockensubstanz } \\ \text { U } & \text { Umdrehung } \\ \text { UV } & \text { Ultraviolett } \\ \text { UVP } & \text { Umweltverträglichkeitsprüfung } \\ \text { V } & \text { Variante } \\ & \end{array}$




\section{$1 \quad$ Einleitung}

\subsection{Problemstellung}

Arzneimittelwirkstoffe werden in der Nutztierhaltung in nennenswertem Umfang eingesetzt; dabei handelt es sich in erster Linie um Antibiotika. 1999 wurden im gesamten EU-Gebiet einschließlich der Schweiz insgesamt $13.200 \mathrm{t}$ Antibiotika verbraucht (FEDESA 2001). Auf die Humanmedizin entfielen $8.500 \mathrm{t}$, als Tierarzneimittel wurden 3.900 t Antibiotika verabreicht. 800 t Antibiotika wurden als Leistungsförderer eingesetzt. Mit dem EU-weiten Verbot aller antibiotisch wirksamen Futterzusatzstoffe bis 2006 wird dieser Einsatz allerdings in Zukunft keine Rolle mehr spielen (KOM 2002). Eine im Vergleich zu Angaben der FEDESA (2001) detailliertere Auflistung des Gesamtverbrauches an Tierarzneimitteln z.B. hinsichtlich einzelner Wirkstoffe und Tierarten bzw. Nutzungsrichtungen ist weder EU- noch deutschlandweit verfügbar, da keine zentrale Erfassung der Daten erfolgt.

Die potenziellen Eintragsquellen von Antibiotika in die Umwelt sind vielfältig: nach Verabreichung beim Tier werden sie in zum Teil aktiver und unveränderter Form sowie als aktive Metabolite durch den tierischen Organismus ausgeschieden. Somit kann über Wirtschaftsdünger wie Gülle oder Festmist ein Eintrag dieser Stoffe in Böden stattfinden (Abbildung 1).

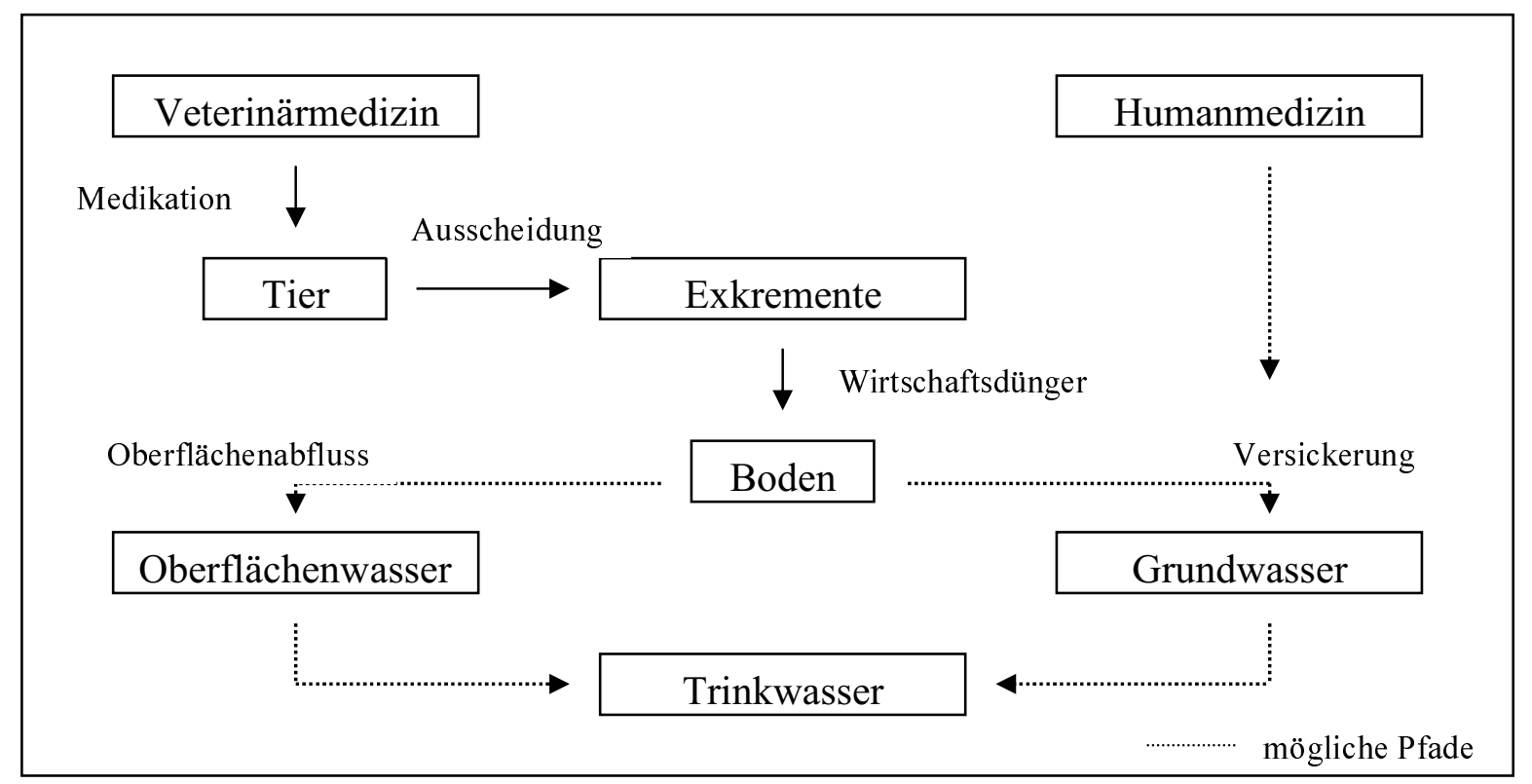

Abbildung 1: Eintragspfade für Antibiotika in die Umwelt

Eine weitere Eintragsquelle in die Umwelt ist in der Aquakultur zu sehen, bei der die Antibiotika im Bedarfsfall direkt in die Gewässer ausgebracht werden. Auch über den 
Pflanzenbau erfolgt ein Eintrag: seit den 1950er Jahren werden Antibiotika als Pflanzenschutzmittel vor allem bei der Produktion von Obst, Gemüse und Zierpflanzen eingesetzt. McManus et al. (2002) schätzten den Verbrauch in den USA zu diesem Zweck auf $0,5 \%$ des gesamten Antibiotika-Verbrauchs, der für die USA mit ca. $10.000 \mathrm{t}$ angegeben wird. Neueste Untersuchungen von Hamscher et al. (2003) wiesen einen Eintrag von Antibiotika sogar über Stäube aus der Schweine- und Geflügelhaltung nach. Über den weiteren Verbleib bzw. das Verhalten der über diese verschiedenen Eintragswege in die Umwelt eingebrachten Antibiotika gibt es derzeit nur wenig Kenntnis.

Verschiedene Erhebungen ergaben, dass es sich bei den veterinärmedizinisch angewandten Antibiotika hauptsächlich um Tetrazykline und Sulfonamide handelt (FEDESA 1999; Winckler und Grafe 2000; Grafe 2001; Abbas et al. 2001; Thiele-Bruhn et al. 2003). Es handelt sich bei beiden Wirkstoffgruppen um so genannte Altpräparate. Mit dem Begriff Altpräparate werden Tierarzneimittel umschrieben, die vor dem 01.01.1978 zur Anwendung kamen oder zwischen 1978 und 1998 zugelassen wurden (Winckler und Grafe 2000; Grafe 2001). Vor 1998 unterlagen diese Wirkstoffe keiner Umweltverträglichkeitsprüfung (UVP). Mit Wirkung zum 01.01.1998 ist eine vom Ausschuss für Tierarzneimittel (CVMP) der Europäischen Arzneimittelagentur (EMEA) erarbeitete Leitlinie (EMEA/CVMP-055/96) zur Durchführung einer UVP von neu zuzulassenden Tierarzneimitteln in Kraft getreten. Eine Überarbeitung erfolgte im Jahr 2000 (EMEA/CVMP/VICH/592/98; VICH GL 6 -Ecotoxicity Phase I) (EMEA 2000). Unter Berücksichtigung der eingesetzten Menge des Wirkstoffes, der biologischen Abbaubarkeit, der Adsorption im Boden und anderer Parameter wird im ersten Schritt ein Expositionsszenario erstellt und die $\mathrm{zu}$ erwartende Konzentration im Boden $\mathrm{PEC}_{\text {soil }}$ ermittelt (Phase I). Bei der Überschreitung bestimmter Schwellen- bzw. Triggerwerte $\left(\mathrm{PEC}_{\text {soil }}>100 \mu \mathrm{g} \mathrm{kg}^{-1} ; \mathrm{PEC}_{\text {water }}>1 \mu \mathrm{g} \mathrm{l}^{-1}\right)$ sind weitere Untersuchungen zur Ökotoxizität, zum Abbau im Boden bzw. Wasser sowie zur Adsorption erforderlich (Phase II).

Da sich die Leitlinie jedoch nur auf die Zulassung neuer Tierarzneimittel bezieht, werden Altpräparate diesbezüglich nicht untersucht. Somit besteht ein erheblicher Mangel an umweltrelevanten Informationen über das Verhalten der Altpräparate nach der Ausscheidung durch das Tier. Auf die Bewertungslücke hinsichtlich der Umweltrelevanz dieser Wirkstoffe wurde bereits an verschiedener Stelle hingewiesen (Montforts et al. 1999; Winckler und Grafe 2000; Tolls 2001; Hamscher et al. 2002; Höper et al. 2002; Thiele-Bruhn et al. 2003). 
Nur durch ein genaueres Wissen um die Wirkungen der Altpräparate in der Umwelt ist eine Risikobewertung möglich und können mögliche Präventivmaßnahmen eingeleitet werden. Wichtige Kriterien für eine Risikobewertung von Antbiotika hinsichtlich des Bodenschutzes sind verlässliche Angaben zur Expositionsabschätzung, zu potentiellen Austrägen ins Grundwasser oder $\mathrm{zu}$ den Auswirkungen auf die mikrobielle Bodenbiozönose. Einen weiteren wichtigen Aspekt stellt die mögliche Induktion und Verbreitung von Antibiotikaresistenzen im Boden undin anderen Ökosystemen dar.

\subsection{Zielsetzung}

Ziel dieser Arbeit ist daher, Erkenntnisse hinsichtlich Umweltverhalten, Exposition und potentiellen Austrägen ins Grundwasser am Beispiel von ausgewählten Alpräparaten aus den Wirkstoffgruppen der Tetrazykline und Sulfonamide zu gewinnen, um einen Beitrag zur Abschätzung der Umweltgefährdung zu leisten.

In einer früheren Studie konnten Winckler und Grafe (2000) eine ausgeprägte Persistenz von Tetrazyklin in Schweinegülle nachweisen. Auf diesen ersten Ergebnissen aufbauend lag ein Schwerpunkt der vorliegenden Arbeit auf der Persistenz von Tetrazyklin in Masthähnchenfestmist, da neben der Schweinemast auch in der Geflügelmast ein erheblicher Einsatz von Tetrazyklinen stattfindet (Grafe 2001). Weiterhin sollte als Ergänzung zu einer Untersuchung von Grafe (2001) eine Erhebung zu den Gehalten an ausgewählten Tetrazyklinen und Sulfonamiden in Gülleproben aus der Praxis durchgeführt werden. Ein Vergleich zwischen zwei Probennahmejahren sollte mögliche Veränderungen in der Verschreibungspraxis aufzeigen. Im Hinblick auf den Eintragspfad Boden-Wasser wurde die Mobilität von Tetrazyklin und Sulfadiazin in verschiedenen Böden nach Ausbringung über Gülle im Gefäß- und Lysimetermaßstab untersucht.

Folgende Fragestellungen wurden bearbeitet:

- Wie verhält sich der Wirkstoff Tetrazyklin bei der Lagerung von Masthähnchenfestmist, welche Einflussfaktoren auf den Abbau gibt es?

- Wie sieht die tatsächliche Belastung der Schweinegülle mit Tetrazyklin- und Sulfonamidrückständen aus? Gibt es Unterschiede in der Belastung der Schweinegülle mit ausgewählten Antibiotika in den Winterhalbjahren 1999/2000 und 2001/2002?

- Sind die Wirkstoffe Tetrazyklin und Sulfadiazin im Boden mobil und gbt es Unterschiede in der Verlagerungsneigung bei verschiedenen Böden? 


\section{Literaturübersicht}

\subsection{Begriffsbestimmungen}

Tierarzneimittel sind Medikamente, die in der Veterinärmedizin in therapeutisch wirksamer Dosierung verabreicht werden und verschreibungspflichtig durch den Tierarzt sind. Der Begriff Arzneimittel hat laut Richtlinie 65/65/EWG folgende Definition:

Arzneimittel sind alle Stoffzusammensetzungen, die als Mittel zur Heilung oder zur Verhütung menschlicher oder tierischer Krankheiten bezeichnet werden; alle Stoffe oder Stoffzusammensetzungen, die dazu bestimmt sind, im oder am menschlichen oder tierischen Körper zur Erstellung einer ärztlichen Diagnose oder zur Wiederherstellung, Besserung oder Beeinflussung der menschlichen oder tierischen Körperfunktionen angewandt zu werden. (Tier-)Arzneimittel unterliegen dem Arzneimittelgesetz.

Die in dieser Arbeit näher untersuchten Tetrazykline sind den Tierarzneimitteln zuzuordnen und gehören zur Gruppe der Antibiotika (Kroker et al. 1996). Als BreitbandAntibiotika werden sie sowohl in der Human- als auch in der Veterinärmedizin eingesetzt. Sie werden von Streptomycesarten gewonnen. Der Begriff Antibiotika leitet sich aus der Entstehung derselben in verschiedenen Mikroorganismen wie Bakterien oder Pilzen ab (Kroker et al. 1996). Die Bezeichnung Breitband-Antibiotika wird für solche Substanzen verwendet, die ein sehr weites Wirkungsspektrum haben, also sowohl gegen grampositive als auch gramnegative Bakterien eingesetzt werden können. Demzufolge ist ihr Einsatz in der therapeutischen Praxis sehr häufig. Unter dem Oberbegriff Chemotherapeutika werden sowohl die natürlichen als auch die synthetisch hergestellten Antibiotika mit vergleichbarer Wirkung zusammengefasst (Kroker et al. 1996).

In der vorliegenden Arbeit wurden neben den Tetrazyklinen auch Sulfonamide berücksichtigt, um ein weiteres häufig eingesetztes Tierarzneimittel in die Untersuchungen zur Persistenz und Mobilität mit einzubeziehen. Sulfonamide stellen Derivate des $p$ Amino-Benzoe-Sulfonamids (Sulfanilamid) dar. Sie bestehen aus einem Benzolkern mit einer Amino- und einer Sulfonamidgruppe (Kroker et al. 1996). Sulfonamide gehören wie die Tetrazykline zu den Breitband-Antibiotika.

Neben den Tetrazyklinen und Sulfonamiden gibt es noch weitere wichtige Antibiotikagruppen. Unter anderem sind die Aminoglykoside, $\beta$-Laktame, Polymyxine, 
Chinolone, Makrolide und Lincosamide zu nennen. Die Gruppen unterscheiden sich in der Wirkungsart und dem Wirkungsort in der Bakterienzelle.

Antimikrobiell wirksame Substanzen werden auch als Futterzusatzstoffe verwendet. Im Gegensatz zu Tierarzneimitteln unterliegen sie dem Futtermittelgesetz. Pharmakologisch wirksame Futterzusatzstoffe werden in subtherapeutischen Dosierungen verfüttert, um leistungssteigernde Effekte wie z.B. eine erhöhte Mastleistung beim Tier zu erzielen. Antimikrobiell wirksame Substanzen finden sich sowohl bei Tierarzneimitteln als auch unter den Futterzusatzstoffen. Allerdings werden als Futterzusatzstoffe keine Antibiotika verwendet, die auch in der Humanmedizin oder als Tierarzneimittel zugehssen sind.

Als pharmakologisch wirksame Futterzusatzstoffe sind derzeit noch in der EUzugelassen (Stand Frühjahr 2004): Avilamycin, Flavophospholipol, Monensin-Natrium, SalinomycinNatrium. Da der Einsatz dieser Substanzen in den vergangenen Jahren aufgund potentieller Kreuzresistenzen zu therapeutisch eingesetzten Antibiotika zunehmend kritisch betrachtet wurde, hat die EU im Frühjahr 2002 ein Verbot der vier noch zugelassenen antimikrobiell wirkenden Futtermittelzusatzstoffe ab 2006 vorgeschlagen (KOM 2002). Einzige Ausnahme ist Avilamycin, das für Puten weiterhin Anwendung finden darf (Anonymous 2003). Im Entwurf zur diesbezüglichen Verordnung wurde weiterhin festgelegt, dass Zusatzstoffe grundsätzlich nur noch für zehn Jahre zugelassen werden; nach Ablauf dieses Zeitraums muss jeweils eine Neuzulassung beantragt werden. Firmen, die bereits nach geltenden Vorschriften zugelassene Futtermittelzusatzstoffe in den Verkehr bringen, haben innerhalb der nächsten sieben Jahre eine Neubewertung und Neuzulassung ihrer Produkte zu beantragen. Dazu müssen die Unternehmen nachweisen, dass der Zusatzstoff positive Wirkungen auf das Tier hat und dass für die Gesundheit von Mensch und Tier sowie für die Umwelt keine Gefahr besteht(KOM 2002).

\subsection{Physikochemische und pharmakokinetische Eigenschaften ausgewählter Antibiotika}

Antibiotika werden hinsichtlich ihrer Wirkung auf das Bakterienwachstum als bakterizid bzw. bakteriostatisch bezeichnet. Die erfolgreiche Wirkung gegen Bakterien ist von der Konzentration der antibakteriell wirkenden Substanz am Infektionsort abhängig. Sie muss hoch genug sein, um eine bakterizide oder bakteriostatische Wirkung erzielen zu können. Die Anwendung bakteriostatisch wirkender Antibiotika setzt ein funktionstüchtiges Abwehrsystem im zu behandelnden Organismus voraus, da der Beginn der klinischen Wirksamkeit sich erst nach zwei bis drei Tagen Behandlungsdauer erkennen läßt. Bei 
bakterizid wirkenden Antibiotika tritt ein sofortiger Zelltod des zu bekämpfenden Keimes ein, eine Wirksamkeit ist in diesem Fall nach ein bis zwei Tagen zu beobachten (Kroker et al. 1996).

\section{Tetrazykline}

Tetrazykline bestehen aus einem Naphthacen-Kern (Abbildung 2) und sind kaum wasserlöslich, ihre dazugehörigen Hydrochloride weisen dagegen eine bessere Wasserlöslichkeit auf. Sie sind relativ stabil in Säure, aber nicht im basischen Milieu und formen Salze in beiden Medien (Halling-Sørensen et al. 2002). Die verschiedenen Derivate innerhalb der Tetrazyklingruppe wie z.B. Chlor- oder Oxytetrazyklin unterscheiden sich lediglich durch kleine Veränderungen an der Molekülstruktur(Abbildung 2). Tetrazykline zeigen eine hohe Affinität zu polyvalenten Kationen (z.B. Magnesium, Kalzium, Eisen) unter der Bildung von Chelatverbindungen. Aus diesem Grund können sich Tetrazykline in Zähnen, Knochen und Knorpel ablagern, vor allem, wenn sie in der Wachstumsphase verabreicht werden (Brüggemann et al. 1966). Die orale Bioverfügbarkeit ist stark abhängig vom Füllungszustand des Magens und der Zusammensetzung der verabreichten Nahrung. Sie beträgt durchschnittlich ca. 50 \% (Kroker 2002).

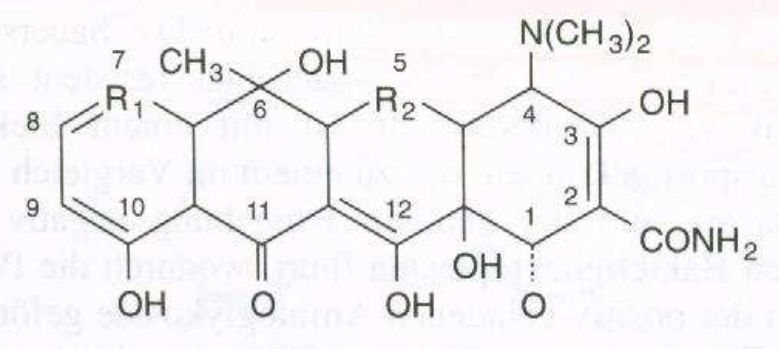

$$
\begin{aligned}
& \text { Tetracyclin } \\
& \begin{array}{ll}
\mathrm{R}_{1}=\mathrm{Cl} & \text { Chlortetracyclin } \\
\mathrm{R}_{2}=\mathrm{OH} & \text { Oxytetracyclin }
\end{array}
\end{aligned}
$$

Abbildung 2: $\quad$ Struktur der Tetrazykline $(\mathrm{R}=\mathrm{Rest})($ Kroker et al. 1996)

Tetrazykline werden je nach Tierart zu veränderlichen Anteilen in aktiver Form renal durch globuläre Filtration (Niere) oder über die Fäzes ausgeschieden. Die Anteile der ausgeschiedenen an den verabreichten Substanzmengen sind je nach Applikationsart und verabreichter Tetrazyklinsubstanz unterschiedlich, können aber bis zu $80 \%$ der eingesetzten Tetrazyklinmenge betragen (Kroker 1983; Winckler und Grafe 2000). Tetrazykline können oral über das Trinkwasser in gelöster Form (vor allem bei Geflügel) bzw. als Pulver über das Futter sowie parenteral verabreicht werden. Die enterale 
Resorption sowie die Proteinbindung und die Urinausscheidung ist je nach Tierart und verabreichtem Tetrazyklin unterschiedlich (Tabelle 1).

Tabelle 1: Pharmakokinetische Daten von Tetrazyklinen(Kroker et al. 1996)

\begin{tabular}{|c|c|c|c|c|}
\hline Substanz & $\begin{array}{c}\text { Enterale } \\
\text { Resorption } \\
(\%)\end{array}$ & $\begin{array}{c}\text { Halbwertszeit } \\
\text { (h) }\end{array}$ & $\begin{array}{c}\text { Proteinbindung } \\
\qquad(\%)\end{array}$ & $\begin{array}{l}\text { Urinaus- } \\
\text { scheidung } \\
(\%)\end{array}$ \\
\hline \multirow[t]{3}{*}{ Tetrazyklin } & \multirow{3}{*}{$\begin{array}{r}\text { bis } 50 \text { (Hund) } \\
\text { bis } 80 \text { (Mensch) }\end{array}$} & 5 (Hund) & 36 (Rind) & 56-85 (Hund) \\
\hline & & 10 (Kalb) & 70-80 (Hund) & 40 (Mensch) \\
\hline & & 10 (Mensch) & 25-55 (Mensch) & \\
\hline \multirow[t]{3}{*}{ Chlortetrazyklin } & 24 (Hund) & 5 (Hund) & 38 (Rind) & \multirow{3}{*}{$\begin{array}{r}\text { ca. } 20 \\
\text { (Mensch) }\end{array}$} \\
\hline & 47 (Kalb) & 9 (Kalb) & 61 (Hund) & \\
\hline & 25-30 (Mensch) & 5-6 (Mensch) & ca. 50 (Mensch) & \\
\hline \multirow[t]{3}{*}{ Oxytetrazyklin } & 60 (Mensch) & 3 (Huhn) & $<30$ & 30 (Mensch) \\
\hline & & bis 11 (Pferd) & & \\
\hline & & 8-10 (Mensch) & & \\
\hline
\end{tabular}

Es gelten Dosierungsmengen für Tetrazykline von etwa $20-50 \mathrm{mg} / \mathrm{kg}$ je Tag für Kälber, Schweine, Hunde und für Huhn/Taube $50-100 \mathrm{mg} / \mathrm{kg}$ je Tag. Die Wartezeiten betragen nach parenteraler Verabreichung 21 (Oxytetrazyklin) bzw. 10 (Tetrazyklin) Tage, nach oraler Gabe 14 Tage (Kroker et al. 1996).

Bei feuchter Lagerung der Tetrazykline, Lichteinwirkung und hohen Temperaturen bilden sich über Dehydratation Epi-, Epianhydro- oder Anhydroprodukte, die zwar nicht antibiotisch wirksam sind, sich aber unter bestimmten Umständen wieder zur Hauptsubstanz umwandeln können (Kroker et al. 1996; Kroker 1997; Kühne et al. 2002). Rückstände lassen sich in Niere, Leber, Muskulatur, Milch und Eiern finden (Kroker 2002). Bei zum Verzehr bestimmten Tieren muss vor der Schlachtung eine Wartezeit eingehalten werden. Tetrazykline wirken bakteriostatisch. Indikationsgebiete sind Infektionen der Atemwege, des Urogenital und des Magen-Darm-Traktes sowie der Haut (Kroker et al. 1996).

\section{Sulfonamide}

Sulfonamide sind nahezu unlöslich in Wasser, formen aber Salze im stark sauren oder basischen Milieu, welche gut wasserlöslich sind (Ingerslev und HallingSørensen 2000) (Tabelle 2). Im Stoffwechsel des Warmblüterorganismus kommen Sulfonamide in drei Hauptformen vor: als freies sowie als an Plasmaproteine gebundenes Sulfonamid und als 
acetylierte Verbindung. Letztere bildet das Hauptabbauprodukt (Kroker et al. 1996). Der an Proteine gebundene Teil kann nicht antibakteriell wirken. Wirksam ist nur das freie, nicht acetylierte Sulfonamid (Abbildung 3). Die Ausscheidung erfolgt hauptsächlich durch die Niere. Als andere, jedoch mengenmäßig unbedeutendere Ausscheidungswege kommen Galle, Pancreas- und Magensaft, Bronchialsekret, Speichel und Milch in Frage (Kroker et al. 1996).

Wie auch bei den Tetrazyklinen werden die Sulfonamide größtenteils in unveränderter, also chemotherapeutisch aktiver Form ausgeschieden. Ein Teil der Sulfonamide wird als unwirksame Metaboliten, vor allem Acetylderivate, Glucuronide und Konjugate mit Schwefelsäure, über den Harn abgegeben (Kroker et al. 1996). Studien von Berger et al. (1986) und Langhammer (1989) wiesen bei Schweinen Ausscheidungsmengen von 40 $90 \%$ der eingesetzten Substanz nach. Auch bei den Sulfonamiden variieren die Mengen je nach Tier- und Applikationsart.

Im Allgemeinen werden Sulfonamide, vor allem auch ihre wasserlöslichen Natrium Salze, nach oraler Applikation gut resorbiert. Sulfadiazin hat eine Halbwertszeit in Plasma und Blut von acht Stunden beim Schwein sowie zehn bis 24 Stunden beim Menschen. Dagegen weist Sulfamethazin eine kürzere Halbwertszeit beim Menschen auf (3 - 14 Stunden) und eine längere beim Schwein (9 - 16 Stunden) (Kroker et al. 1996).

In der praktischen Therapie werden Sulfonamide häufig in verschiedenen Kombinationen untereinander angewendet. Die Tagesdosis variiert je nach verwendetem Sulfonamid. Bei Sulfamethazin beträgt sie bspw. 50 bis $100 \mathrm{mg} / \mathrm{kg}$ je Tag und ist damit mit der der Tetrazykline vergleichbar. Die Wartezeiten betragen bei parenteraler Applkation je nach Tierart sechs (Schaf) bis zwölf (Huhn) Tage sowie nach oraler Gabe acht (Schaf) bis 14 (Huhn) Tage (Kroker et al. 1997).
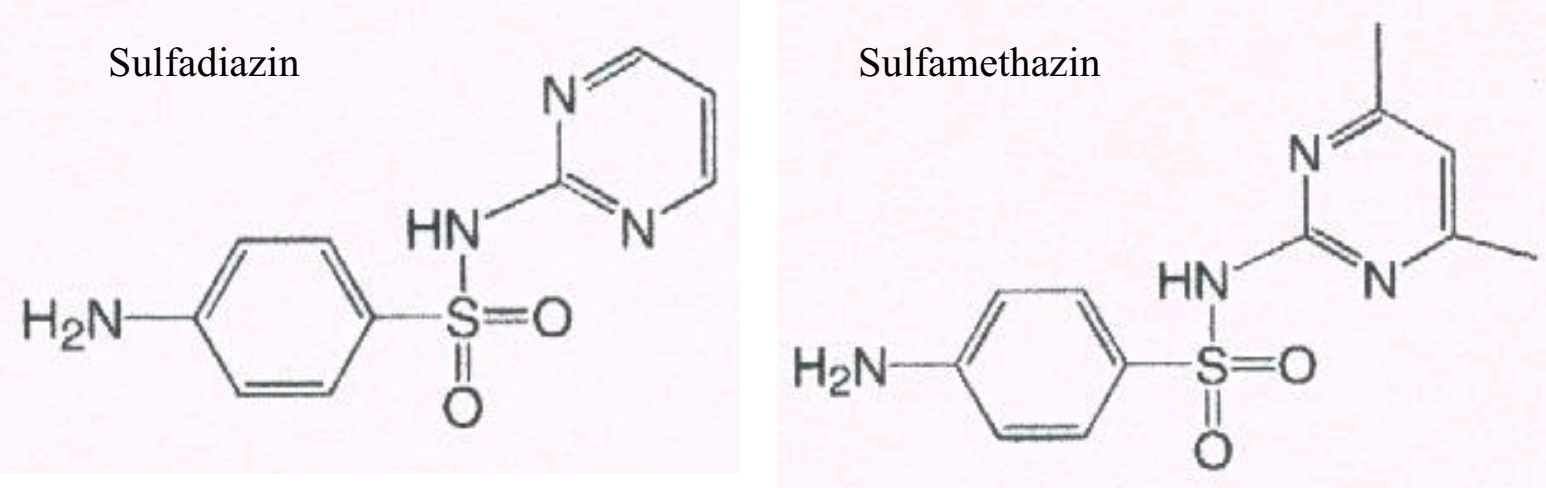

Abbildung 3: Struktur ausgewählter Sulfonamide (Haller et al. 2002) 
Indikationen für die Anwendung von Sulfonamiden sind u.a. Infektionen wie MagenDarm-Entzündung, Salmonellen, Toxoplasmose und Entzündungen des Urogenitaltraktes. Hier hat sich vor allem der synergistische Effekt von Trimethoprim bewährt, einem Antimetaboliten, welcher die Sulfonamide in der Wirksamkeit potenziert(Sequentialeffekt auf die bakterielle und protozoale Folsäuresynthese). Rückstände der Sulfonamide und von Trimethoprim finden sich bei Nichteinhaltung der vorgeschriebenen Wartezeiten in der Muskulatur, im Fett, in Leber und Niere (Kroker 2002).

In Tabelle 2 sind einige der umweltrelevanten Eigenschaften der in der vorliegenden Arbeit untersuchten Antibiotika aufgeführt. Die Henry-Konstante beschreibt das Verhältnis zwischen Partialdruck in der Luft und der Gleichgewichtskonzentration im Wasser Sie ist abhängig von Druck, Temperatur und den Inhaltsstoffen des Wassers. Die aufgeführten Antibiotika weisen eine geringe Flüchtigkeit von unter $0,003 \mathrm{~Pa}_{1 \mathrm{~mol}^{-1}}$ auf, so dass mit keiner nennenswerten Volatilität zu rechnen ist (Römpp 1995). Die Säurekonstante $\mathrm{pK}_{\mathrm{s}}$ beschreibt den pH-Wert, bei dem die Säuregruppen (H-Ionen) der Wirkstoffe zu $50 \%$ dissoziiert sind. Der n-Octanol-Wasser-Verteilungskoeffizient $\left(\log \mathrm{K}_{\text {ow }}\right)$ ist ein Maß für die Fettlöslichkeit, also die Verteilung einer Substanz zwischen polarer (Wasser) und unpolarer (n-Octanol) Phase. Die untersuchten Wirkstoffe weisen einen niedrigen $\log \mathrm{K}_{\mathrm{ow}}$ auf, so dass mit einer guten Wasserlöslichkeit und somit einem geringen Bioakkumulationspotential zu rechnen ist.

Tabelle 2: Umweltrelevante physiko-chemische Eigenschaften wie Löslichkeit, Volatilität, Säurekonstante $\left(\mathrm{pK}_{\mathrm{s}}\right)$ und Oktanol-Wasser-Verteilungskoeffizient $\left(\log \mathrm{K}_{\mathrm{ow}}\right)$ ausgewählter Veterinärantibiotika (Römpp 1995; Thiele-Bruhn 2003a)

\begin{tabular}{lrrrrrr}
\hline Substanz & Summenformel & Molmasse & $\begin{array}{c}\text { Löslich- } \\
\text { keit } \\
\left(\mathbf{~ g ~ m o l ~}^{-1}\right)\end{array}$ & $\begin{array}{c}\text { Henry- } \\
\text { Konstante }\end{array}$ & $\mathbf{p K}_{\mathbf{s}}$ & $\begin{array}{c}\text { log } \\
\left(\mathbf{P a ~ l ~ m o l}^{-1}\right)\end{array}$ \\
\hline Tetrazyklin & $\mathrm{C}_{22} \mathrm{H}_{24} \mathrm{~N}_{2} \mathrm{O}_{8}$ & 444 & 230 & $4,7 \times 10^{-22}$ & $3,3 / 7,7 / 9,5$ & $-1,3$ \\
Chlortetrazyklin & $\mathrm{C}_{22} \mathrm{H}_{23} \mathrm{CIN}_{2} \mathrm{O}_{8}$ & 479 & 630 & $3,5 \times 10^{-22}$ & $3,3 / 7,8 / 9,3$ & $-0,6$ \\
Oxytetrazyklin & $\mathrm{C}_{22} \mathrm{H}_{24} \mathrm{~N}_{2} \mathrm{O}_{9}$ & 460 & 310 & $1,7 \times 10^{-23}$ & $3,3 / 7,3 / 9,1$ & $-0,9$ \\
Sulfamethazin & $\mathrm{C}_{12} \mathrm{H}_{14} \mathrm{~N}_{4} \mathrm{O}_{2} \mathrm{~S}$ & 278 & 1500 & $3,1 \times 10^{-11}$ & $2,8 / 7,6$ & 0,9 \\
Sulfadiazin & $\mathrm{C}_{10} \mathrm{H}_{10} \mathrm{~N}_{4} \mathrm{O}_{2} \mathrm{~S}$ & 250 & 270 & - & - & $-0,09$ \\
\hline
\end{tabular}




\subsection{Persistenz von Tierarzneimitteln in verschiedenen Medien}

Die zum Zeitpunkt der Ausbringung vorliegenden Gehalte der Antibiotika in den Wirtschaftsdüngern sind von einer Reihe von Einflussfaktoren abhängig. Eine wichtige Rolle spielt vor allem bei Gülle der Verdünnungseffekt durch bereits vorhandene unbelastete Gülle sowie die weitere Vermischung mit antibiotikafreien Ausscheidungen im Anschluss an den Behandlungszeitraum. Dieser Effekt ist aber auch bei Festmist zu beachten, da hier neben der Zufuhr von unbelastetem Mist auch Stroh zugeführt wird. Als Einflussfaktoren auf das Lagerverhalten von Antibiotika in Wirtschaftsdüngern kommen in erster Linie Substrattemperatur, Sauerstoffversorgung bzw. Sauerstoffeintrag (Langhammer 1989) und physikalisch-chemische Eigenschaften der Matrix wie der pHWert (Kroker 1983) in Frage. Es existieren zu dieser Fragestellung bislang nur wenige Untersuchungen, die im Folgenden vorgestellt werden.

In der Regel werden Wirtschaftsdünger nach unterschiedlicher Lagerungszeit auf den Boden aufgebracht. Festmist aus der Masthähnchenproduktion wird häufig auf Mistlagerstätten oder am Feldrand zwischengelagert bzw. während der Vegetationsperiode sofort als Dünger ausgebracht. Gülle wird unterhalb der Spaltenböden gelagert bzw. in einem Fließmistsystem aus dem Stall über eine Vorgrube in einen Güllebehälter gefördert, aus dem dann in unregelmäßigen Abständen die Gülle zum Ausbringen abgepumpt wird. Je nach Jahreszeit, Witterungslage und Lagerungskapazität variieren die Lagerzeiten daher von einigen Wochen bis zu über sechs Monaten. Die Ausbringung von Wirtschaftsdüngern auf landwirtschaftlich genutzte Flächen ist in der Düngeverordnung geregelt (DüngeVO 1996). So dürfen Wirtschaftsdünger nicht auf gefrorenen Boden sowie zwischen Mitte November und Mitte Januar ausgebracht werden.

Auslöser für die immer noch anhaltende Diskussion um Rückstände von Tierarzneimitteln in der Umwelt war in den 80er Jahren des 20. Jahrhunderts u.a. der Nachweis der die Dungfauna schädigenden Wirkung von Ivermectin (Handelsname Ivomec ${ }^{\circledR}$ ), einem zur Substanzgruppe der Avermectine zählenden Breitband-Antiparasitikum. Wall und Strong (1987) konnten nach Verabreichung von Ivermectin als Bolus (kontinuierliche Abgabe von $40 \mu \mathrm{g} \mathrm{kg}^{-1}$ Lebendmasse je Tag) einen Einfluss auf die dungabbauenden Insekten Coleoptera, Diptera, Anneliden und Centipedes nachweisen. Es zeigten sich eine Verminderung der Dungkäferanzahl sowie ein unvollständiger Abbau der Fladen. Auch Schaper und Liebisch (1991) untersuchten Kuhfladen von mit Ivermectin behandelten Kühen, stellten aber bei den beobachteten Insekten (Diptera: Muscidae-Fliegen, Sepsidae- 
Schwingfliegen) bezüglich Besiedelung und Umsetzung der Kuhfladen keine Unterschiede fest. Allerdings nahm die Schlupfrate von Diptera um $60 \%$ ab.

Schließlich wiesen Strong et al. (1996) nach, dass Kuhfladen, die von mit Ivermectin behandelten Tieren stammten, eine verminderte Insektenanzahl von Coleoptera und Diptera im Gegensatz zu mit Fenbendazol ${ }^{1}$ durchsetzten Fladen zeigten. Auch war die Kuhfladenbeschaffenheit nach sechs Wochen bei Ivermectin noch fest und kompakt, bei Fenbendazol dagegen schon krümelig und körnig.

Jagnow (1977) führte schon in den 70er Jahren des 20. Jahrhunderts Untersuchungen zum Verhalten von antibiotisch wirkenden Substanzen in Wirtschaftsdünger durch. Mittels mikrobiologischer Testverfahren und einem in-vitro-Ansatz wurden die Antibiotika Tetrazyklin, Flavophospholipol, Zink-Bacitracin und Spiramycin auf ihre Stabilität in Hühnerkot getestet. Bis auf Zink-Bacitracin, das auf $25 \mathrm{mg} \mathrm{kg}^{-1}$ in Hühnerkot eingestellt wurde, wurden die anderen Wirkstoffe mit $10 \mathrm{mg} \mathrm{kg}^{-1}$ geprüft. Zink-Bacitracin war nach neun Tagen vollständig inaktiviert, Flavophospholipol erst nach 17 Wochen, Spiramyän dagegen wurde in den ersten zwei Wochen rasch $(50-60 \%)$ abgebaut, später jedoch deutlich langsamer, so dass nach vier Wochen noch ca. 30 \% vorhanden waren. Der Abbau von Tetrazyklin verlief ebenfalls relativ langsam; selbst nach zwölf Wochen Inkubationszeit war noch etwa ein Drittel der antibakteriellen Aktivität vorhanden. In Varianten, in denen ein Kot-Boden-Gemisch getestet wurde (im Verhältnis Kot zu Boden von 1:3), erfolgte der Abbau aller getesteten Antibiotika deutlich schneller.

Auch Warman und Thomas (1980) untersuchten die mikrobielle Aktivität von Chlortetrazyklin in Hühnerkot gemischt mit Boden. Sie stellten fest, dass Chlortetrazyklin in Konzentrationen von 0,1 bzw. $0,6 \mu \mathrm{g} \mathrm{g}^{-1}$ über 24 Tage keinen Effekt auf die Bodenatmung hatte. Die Autoren vermuteten aufgrund dieser Ergebnisse, dass Chlortetrazyklin bei in den Boden eingearbeitetem Hühnerkot nicht mehr nachweisbar sei.

Neuere Untersuchungen wiederum von Gavalchin und Katz (1994) ergaben eine Reduktion der mikrobiellen Aktivität von Bacillus cereus in mit Hühnerkot im Verhältnis 5:95 mit Boden vermischtem Medium um $44 \%$ nach 30 Tagen bei $30{ }^{\circ} \mathrm{C}$ Bodentemperatur. Bei 4 bzw. $20^{\circ} \mathrm{C}$ waren noch 88 bis $100 \%$ Restaktivität feststellbar, was als ein Hinweis auf die ausgeprägte Temperaturabhängigkeit beim Abbau von Chlortetrazyklin interpretiert wurde.

1 Anmerkung: Fenbendazol ist ebenfalls ein Antiparasitikum, jedoch aus der Wirkstoffgruppe der Benzimidazole. 
Neben Hühnerkot wurde auch in Rindermist die Stabilität von Antibiotika untersucht. Elmund et al. (1971) gaben an junge Rinder 70 mg/Tier/Tag Chlortetrazyklin. Im frischen Rindermist konnten sie über einen Bioassay $14 \mu \mathrm{g} \mathrm{g}^{-1}$ Chlortetrazyklin nachweisen, im gealterten Mist immer noch 0,34 $\mu \mathrm{g} \mathrm{g}^{-1}$. Sie ermittelten Halbwertszeiten von sieben Tagen bei $37^{\circ} \mathrm{C}$ Inkubationstemperatur, bei 4 bis $28^{\circ} \mathrm{C}$ Halbwertszeiten von über vier Wochen. In unbehandeltem Rindermist wurde deutlich mehr organische Masse abgebaut als in mit Chlortetrazyklin versetztem Mist.

Diese Ergebnisse wurden durch jüngste Studien von De Liguoro et al. (2003) bestätigt: in dieser Untersuchung wurde $60 \mathrm{mg} \mathrm{kg}^{-1}$ Oxytetrazyklin je Tag bzw. $20 \mathrm{mg} \mathrm{kg}^{-1}$ Tylosin je Tag an Kälber verabreicht. Während Tylosin rasch degradierte und schon nach 45 Tagen im Mist nicht mehr nachweisbar war, zeigte Oxytetrazyklin eine Halbwertszeit von 30 Tagen und war auch nach einer fünfmonatigen Reifung des Mistes noch in Konzentrationen von $820 \mu \mathrm{g} \mathrm{kg}^{-1}$ detektierbar. Patten et al. (1980) prüften den Zusammenhang von mit Antibiotika belastetem Rindermist $\left(5,3 \mu \mathrm{g} \mathrm{g}^{-1}\right.$ Tetrazyklin; $11,3 \mu \mathrm{g} \mathrm{g}^{-1}$ Oxytetrazyklin) und der Stickstoffmineralisation im Boden. Sie fanden keinen Unterschied in der Bakterienanzahl und keine Differenz in der Stickstoffmineralisation im Boden bei Einbringung von antibiotikabelastetem und -freiem Rindermist in einer Zeitspanne von 60 Tagen.

Erste Untersuchungen zur Persistenz von antibiotisch wirkenden Tierarzneimitteln in Schweinegülle fanden Mitte der 80er Jahre des 20. Jahrhunderts statt. Der analytische Nachweis der Antibiotika erfolgte ab diesem Zeitraum mit Hilfe der HPLC-Technik. Berger et al. (1986) wiesen bei Schweinen nach, dass einmalig parenteral verabreichtes Chloramphenicol bereits nach 24 Stunden vollständig ausgeschieden war. Ebenfalls verabreicht wurde Sulfadimidin, dessen Ausscheidung nach Medikationsende ähnlich schnell erfolgte. $46 \%$ des verabreichten Sulfadimidins ließen sich in der Gülle wiederfinden, davon anteilig 50\% als N4-Acetyl-Sulfadimidin. Bei der anschließenden Güllelagerung wurde dieser Metabolit durch mikrobiellenzymatische Prozesse zum biologisch aktiven Arzneistoff gespalten, so dass dadurch eine sehr viel höhere und zeitlich länger anhaltende Sulfadimidinbelastung in der Gülle gemessen wurde als zuvor angenommen. Innerhalb von 51 Tagen nahm die Sulfadimidinkonzentration von $253 \mathrm{mg} \mathrm{kg}^{-1}$ auf $82 \mathrm{mg} \mathrm{kg}^{-1} \mathrm{ab}$.

Dieses Ergebnis konnte wenig später durch eine andere Untersuchung bestätigt werden: die achttägige Medikation eines Schweinebestandes mit einem Kombinationspräparat aus den 
Sulfonamiden Sulfamethazin und Sulfadiazol sowie dem Sulfonamid-Synergisten Trimethoprim führte in einer Studie von Langhammer (1989) zu Gesamt-SulfonamidKonzentrationen inklusive konjugierter Metabolite von bis zu $39 \mathrm{mg} \mathrm{kg}^{-1}$ in der Gülle. Die Rückstände bauten sich in fünf Wochen um 40-60\% ab, Trimethoprim war nicht mehr nachweisbar.

Kühne et al. (2000) ermittelten in-vitro Halbwertszeiten für Tetrazyklin in Schweinegülle von neun Tagen bei unbelüfteter bzw. 4,5 Tagen bei belüfteter Gülle. Winckler und Grafe (2000) stellten dagegen unter praxisnäheren Bedingungen (300-1-Behälter) für Tetrazyklin eine hohe Lagerstabilität in Schweinegülle fest. Nach sieben Wochen hatte die Konzentration im günstigsten Fall um 50 \% abgenommen. Die Autoren leiten daraus eine gegenüber Kühne et al. (2000) deutlich längere Halbwertszeit für Tetrazyklin zwischen 46 und 106 Tagen ab. Der Einfluss von Ausgangskonzentration, Umgebungstemperatur und Lufteintrag durch zeitweiliges Aufrühren wurde hier als gering eingeschätzt. Beim Mastschwein wurden Konzentrationen von bis $\mathrm{zu} 800 \mathrm{mg} \mathrm{kg}^{-1}$ in frischen Exkrementen bei praxisüblicher Medikation ermittelt (Grafe 2001). Orientierende Angaben zu den tatsächlich in der Praxis anzutreffenden Tetrazyklin-Konzentrationen in Gülle finden sich ebenfalls bei Grafe (2001). Im Rahmen einer Screening-Untersuchung von 181 Schweinegülleproben auf Tetrazyklin-HCl/4-Epi-Tetrazyklin enthielten $24 \%$ der Proben Rückstände oberhalb der Bestimmungsgrenze von $0,6 \mathrm{mg} \mathrm{kg}^{-1}$. Der mittlere TetrazyklinGehalt betrug $11,4 \mathrm{mg} \mathrm{kg}^{-1}$ bei einer Schwankungsbreite zwischen 0,6 und $66,0 \mathrm{mg} \mathrm{kg}^{-1}$. Leider konnten aufgrund der anonymisierten Probennahme keine Angaben zum Zeitpunkt eines Medikamenteneinsatzes oder der Lagerungsbedingungen der Schweinegüllen erhoben werden.

Haller et al. (2002) wiesen mittels LC-MS-MS in sechs Schweinegülleproben aus Dunggruben in der Schweiz Sulfamethazin-Konzentrationen von maximal $11,3 \mathrm{mg} \mathrm{kg}^{-1}$ einschließlich des Metaboliten N4-Acetyl-Sulfamethazin nach. Die Konzentration des nicht antimikrobiell wirkenden Metaboliten war 2- bis 50-mal niedriger als die der Ausgangssubstanz, wie zuvor schon von Berger et al. (1986) beschrieben. Sulfathiazol war in vier Proben (maximal 12,4 $\mathrm{mg} \mathrm{kg}^{-1}$ ) nachweisbar, Trimethoprim lag nur in einer Probe in sehr niedriger Konzentration vor $\left(<0,1 \mathrm{mg} \mathrm{kg}^{-1}\right)$ (Haller et al. 2002).

Güllen, mit denen niedersächsische Bodendauerbeobachtungsflächen beaufschlagt wurden, enthielten ca. $4 \mathrm{mg} \mathrm{kg}^{-1}$ Tetrazyklin sowie $0,1 \mathrm{mg} \mathrm{kg}^{-1}$ Chlortetrazyklin (Hamscher et al. 2002). Campagnolo et al. (2002) konnten mittels LC/ESI-MS in Schweinegülleproben von 
neun Farmen in den USA Chlor-, Oxy- und Tetrazyklin (Bestimmungsgrenze 0,5 $\mu \mathrm{g}^{-1}$ ) nachweisen. Ebenso lagen Lincomycin (maximal $240 \mu \mathrm{g}^{-1}$ ), Sulfamethazin (maximal $400 \mu \mathrm{g} \mathrm{l}^{-1}$ ), Sulfadimethoxin, Trimethoprim und Erythromycin (alle maximal 2,5 $\mu \mathrm{g}^{-1}$ ) vor. Auch hier wurden wie bei Grafe (2001) keine Daten zur Verschreibungspraxis der Betriebe angegeben.

In einer weiteren Studie wurde Escherichia Coli als Biosensor genutzt zum Nachweis von Chlortetrazyklin (Bestimmungsgrenze $0,03 \mathrm{mg} \mathrm{kg}^{-1}$ ) in den Ausscheidungen von Schweinen (Hansen et al. 2002). Zwei Schweine erhielten $800 \mathrm{mg}$ Chlortetrazyklin je kg Futter über drei Tage. An Tag fünf nach Absetzen des Antibiotikums wurden Konzentrationen bis $\mathrm{zu} 112 \mathrm{mg} \mathrm{kg}^{-1}$ in den Faeces gemessen und noch nach 30 Tagen konnte Chlortetrazyklin im Kot nachgewiesen werden. Weiterhin stellten die Autoren an Tag fünf einen raschen Anstieg der Anzahl tetrazyklinresistenter koliformer Keime fest.

Loke et al. (2002) schließlich zeigten auf, dass bestimmte Tierarzneimittel stark an Partikel in der Schweinegülle adsorbieren. Für Oxytetrazyklin stellten die Autoren heraus, dass die Sorption sehr viel höher ist als der relativ niedrige $\log \mathrm{K}_{\mathrm{ow}}$ Wert der Substanz (siehe Tabelle 2) es vermuten lässt. Allerdings war die Bindung von Oxytetrazyklin an Bodenpartikel noch stärker als an Güllepartikel, was durch Mineral- und Metallkomplexe erklärt wurde, die sich im Boden, nicht aber in der Gülle befanden.

Neueste Untersuchungen von Hamscher et al. (2003) ergaben nun neben der Belastungvon Gülle bzw. Festmist auch eine Kontamination des Stallstaubes mit diversen Antibiotika. Sie untersuchten über mehrere Jahre die Stäube aus einem Schweinestall und fanden in 18 von 20 Proben bis zu fünf verschiedene Antibiotika, darunter Tylosin, Chlor-, Oxy- und Tetrazyklin, Sulfamethazin und Chloramphenicol in GesamtKonzentrationen von 0,2 bis $12,5 \mathrm{mg} \mathrm{kg}^{-1}$ Staub. Die Autoren befürchten dadurch gesundheitliche Risiken für die Landwirte.

Durch sensitivere Nachweisverfahren, die auch sehr geringe Konzentrationen (Mikro- bzw. Nanogrammbereich) detektieren, können verschiedene Tierarzneimittel mittlerweile auch im Medium Wasser nachgewiesen werden. Der Eintragspfad ist hier weitestgehend über die Gülleausbringung auf landwirtschaftliche Flächen zu sehen. Darüber hinaus können Industrieabwässer der Arzneimittelproduzenten $\mathrm{zu}$ einem Eintrag von Pharmaka in Fließgewässer führen, wobei es sich in diesem Fall nicht um Arzneimittel aus der veterinärmedizinischen Anwendung handelt. Wohl aber können Substanzen nachgewiesen 
werden, die sowohl in der Human- als auch in der Veterinärmedizin angewendet werden (Heberer und Stan 1998; Daughton und Ternes 1999).

Bei einer landesweiten Untersuchung von 139 Oberflächengewässern in den USA wurden neben zahlreichen anderen Pharmazeutika wie z.B. Antiasthmatika (Albuterol) und Antidepressiva (Fluoxetin) aus der Humanmedizin auch diverse Veterinär und Humanantibiotika in $27 \%$ der beprobten Flüsse in Konzentrationen von bis zu 0,69 $\mu \mathrm{g} \mathrm{l^{-1 }}$ für Chlortetrazyklin bzw. 0,11 $\mu \mathrm{g}^{-1}$ für Tetrazyklin nachgewiesen (Kolpin et al. 2002). Sulfonamide konnten bis zu 1,9 $\mu \mathrm{gl}^{-1}$ (Sulfamethoxazol) bzw. 0,22 $\mu \mathrm{gl}^{-1}$ (Sulfamethazin) gefunden werden.

Ebenfalls in Flusswasser, allerdings in England, wurden Makrolide, Sulfonamide und Tetrazykline in Konzentrationen bis zu $1 \mu \mathrm{g} \mathrm{l^{-1 }}$ detektiert (Watts et al. 1982). Die mögliche Grund- und Oberflächenwasserbelastung durch Antibiotika wurde von Thiele-Bruhn (2003) dagegen aufgrund der Bodenpassage der Substanzen eher als gering eingeschätzt. Der Eintrag der Substanzen erfolgt in der Regel nicht direkt sondern über Wirtschaftsdünger und damit über den Boden und ist daher eng gekoppelt an die mit der Bodenpassage verbundenen Sorptionsmechanismen. Aus diesem Grund wird im folgenden Kapitel nochmals ergänzend auf das Auftreten von Tierarzneimitteln im Medium Wasser eingegangen.

\subsection{Vorkommen und Mobilität von Tierarzneimitteln in Böden}

Im Boden mobile Tierarzneimittel können z.B. über das Sickerwasser ins Grundwasser gelangen. Es muß daher im Hinblick auf eine ökotoxikologische Bewertung geprüft werden, in welcher Menge diese Stoffe im Boden vorhanden sind sowie ob und wie mobil sie sind. Entscheidend bei der Mobilität der Tierarzneimittel im Boden sind die Sorptionsneigung eines Wirkstoffes bzw. die Mechanismen, die bei der Sorption von Antibiotika an Bodenbestandteile beteiligt sind. Stark sorbierende Substanzen können in der Umwelt, hier speziell im Boden, akkumulieren (Tolls 2001; Höper et al. 2002), wobei in diesem Fall die Verfügbarkeit der Substanzen für die im Boden lebenden Organismen von großer Bedeutung ist.

In Versuchen von Samuelsen et al. (1994), die Rückstände von Oxytetrazyklin über sechs Monate in künstlich angelegten Fischfarm-Sedimenten auf Stabilität (durch HPLCNachweis) und mittels Bacillus cereus auf antibakterielle Aktivität testeten, war die Anfangskonzentration von $50 \mu \mathrm{gg}^{-1}$ im Sediment auch nach sechs Monaten noch 
nachweisbar. Eine antibakterielle Wirkung von Oxytetrazyklin bestand dagegen schon nach einem Monat nicht mehr. Erklärt wird dies durch die Adsorption an Tonpartikel (Sithole und Guy 1987) und durch Anlagerung von Kalzium- und Magnesiumionen. Oxytetrazyklin war demnach gegenüber den Bakterien nicht mehr bioverfügbar, aber dennoch nicht abgebaut.

Neuere Übersichtsartikel stellen die verschiedenen bisher bekannten Adsorptionskoeffizienten ausgewählter Tierarzneimittel zusammen (Tabelle 3). Einige Substanzen sind sehr gut wasserlöslich oder ungeladen, andere Verbindungen sind dagegen hydrophob oder ionisieren bei für Böden typischen pH-Werten. Aus diesen Gründen variieren die Kennwerte der Adsorption $\left(K_{\mathrm{d}}\right)$ von Antibiotika in Böden erheblich. Neben einem hydrophoben Verteilungsverhalten und $\mathrm{pH}$-Abhängigkeit werden daher Ionenbindungen, Wasserstoffbrückenbindungen und Komplexierungen an Partikeloberflächen als Adsorptionsmechanismen vermutet (Tolls 2001).

Tabelle 3: Adsorptionskoeffizienten an die Bodenmatrix $\left(K_{\mathrm{f}}\right)$, an die organische Substanz im Boden $\left(K_{\mathrm{oc}}\right)$ sowie an die Tonfraktion im Boden $\left(K_{\text {Ton }}\right)$ ausgewählter pharmazeutischer Antibiotika in Böden und Wirtschaftsdüngern $\left(K_{\mathrm{oc}}-\right.$ Werte sind normiert auf den organischen Kohlenstoffgehalt) (Tolls 2001; Thiele-Bruhn 2003b)

\begin{tabular}{|c|c|c|c|c|}
\hline $\begin{array}{l}\text { Antibiotikum/ } \\
\text { Klasse }\end{array}$ & Probesubstanz & $\begin{array}{l}K_{f} \\
\left(1 \mathrm{~kg}^{-1}\right)\end{array}$ & $\begin{array}{l}K_{\mathrm{oc}} \\
\left(\mathrm{l} \mathrm{kg}^{-1}\right)\end{array}$ & $\begin{array}{l}K_{\text {Ton }}^{a} \\
\left(1 \mathrm{~kg}^{-1}\right)\end{array}$ \\
\hline \multirow[t]{2}{*}{ Tetrazykline } & Oberböden & $417-1.030$ & $27.800-93.300$ & $10.200-93.300$ \\
\hline & Klärschlamm/Gülle & $78-3.020$ & $190-8.160$ & \\
\hline \multirow[t]{2}{*}{ Sulfonamide } & Oberboden & $0,9-3,5$ & $50-320$ & $4-58$ \\
\hline & Gülle & $17-169$ & $40-400$ & \\
\hline \multirow[t]{3}{*}{ Makrolide } & Oberböden & $8,3-128$ & $550-8.000$ & $210-2.100$ \\
\hline & Tonminerale & & & $0,7-3,9$ \\
\hline & Gülle & $46-240$ & 110 & \\
\hline \multirow[t]{2}{*}{ Fluorchinolone } & Oberböden & $260-5.610$ & $16.500-768.700$ & $140-2.500$ \\
\hline & Klärschlamm & 420 & 1130 & \\
\hline
\end{tabular}

${ }^{\mathrm{a}}$ berechnet nach Angaben bzw. Schätzwerten des Tongehaltes der Böden nach $K_{\mathrm{Ton}}=K_{f} /$ Ton (\%) x 100

Antibiotika werden nach Sithole und Guy (1987) in Böden vor allem an organischen Bodenbestandteilen festgelegt. Rabølle und Spliid (2000) untersuchten das Sorptionsverhalten von Oxytetrazyklin und Tylosin in verschiedenen Bodentypen (Sand, sandiger Lehm, lehmiger Sand). Oxytetrazyklin zeigte eine starke Sorption an 
Bodenpartikel, wobei jedoch die jeweiligen Bodentypen keinen Unterschied machten. Tylosin sorbierte mit steigendem Tonanteil im Boden signifikant stärker an die Bodenfraktion. 60 bis $80 \%$ des Tylosins wurden in sandigem Lehm in $5 \mathrm{~cm}$ Tiefe verlagert, in Sandboden dagegen bis in $25 \mathrm{~cm}$ Tiefe.

In einer weiteren Studie wurde das Sorptionsverhalten von Sulfachlorpyridazin in einem Ton- und einem Sandboden untersucht (Boxall et al. 2002). Die Autoren zeigten, dass das Sulfonamid nach Aufbringung auf Tonboden eine geringe Sorptionsneigung aufwies, da es durch Drainagewasser schnell in Oberflächengewässer transportiert wurde(Konzentration: $590 \mu \mathrm{g}^{-1}$ ). Auf sandigem Boden konnten im Sickerwasser nur Konzentrationen im Bereich der Nachweisgrenze von $250 \mathrm{ng} \mathrm{l}^{-1}$ gefunden werden, was durch die Autoren als schneller Abbau von Sulfonamiden interpretiert wurde.

Neben den erwähnten Sorptionseigenschaften der Antibiotika ist für die Risikoabschätzung von großer Bedeutung, in welchen Mengen die Substanzen in Boden und Wasser (Sicker, Drainage-, Oberflächen-, Grundwasser) vorliegen. Expositionsabschätzungen erbrachten für Tetrazyklin Bodenkonzentrationen von 0,4 bis $0,9 \mathrm{mg} \mathrm{kg}^{-1}$ (Winckler und Grafe 2000), für Makrolid-Antibiotika von 13 bis $67 \mu \mathrm{g} \mathrm{kg}^{-1}$ und für Fluorchinolone von 6 bis $52 \mu \mathrm{g} \mathrm{kg}^{-1}$ (Schüller 1998). Studien, in denen tatsächlich gemessene Werte wiedergegeben werden, existieren zurzeit nur wenige.

Über einen Beobachtungszeitraum von zwei Jahren wurden in Oberböden $(0$ bis $30 \mathrm{~cm}$ Tiefe) in konventionell bewirtschafteten und mit Schweinegülle gedüngten Böden Konzentrationen von 5 bis $17 \mu \mathrm{g} \mathrm{kg}^{-1}$ Chlortetrazyklin sowie $<50$ bis $158 \mu \mathrm{g} \mathrm{kg}^{-1}$ Tetrazyklin festgestellt, in einer Einzelprobe (Güllekruste) sogar $1435 \mu \mathrm{g} \mathrm{kg}^{-1}$ Tetrazyklin (Hamscher et al. 2002) sowie $11 \mu \mathrm{g} \mathrm{kg}^{-1}$ Sulfamethazin (Höper et al. 2002). Die genannten Autoren konnten nachweisen, dass die jährliche Wirkstoffzufuhr an Tetrazyklinen deren Abbaurate übersteigt, was eine Akkumulation von Tetrazyklin im Boden zur Folge hat. Eine Verlagerung in den Unterboden konnte nicht nachgewiesen werden. In Böden milchviehhaltender Betriebe, die Klärschlamm erhalten hatten, konnten dagegen keine Tierarzneimittel festgestellt werden (Höper et al. 2002).

In der gleichen Studie wurden Wasserproben analysiert: auch in mittels Saugsonden aus 80 und $120 \mathrm{~cm}$ Tiefe gewonnenem Bodenwasser sowie in oberflächennahem Grundwasser $(200 \mathrm{~cm})$ war keiner der untersuchten Wirkstoffe nachzuweisen. In Kläranlagenabläufen, Drainagegräben und Oberflächengewässern dagegen waren es Hirsch et al. (1998) möglich, von 18 untersuchten antibiotischen Wirkstoffen (human- und 
veterinärmedizinisch eingesetzte Substanzen) fünf in Konzentrationen von weniger als $1 \mu \mathrm{g} \mathrm{kg}^{-1}$ nachzuweisen, darunter das Sulfonamid Sulfamethoxazol sowie Trimethoprim. $\mathrm{Zu}$ ähnlichen Ergebnissen gelangten Hartig et al. (1999) bei der Untersuchung mittels LCMS-MS von fünf Oberflächengewässerproben auf 13 Sulfonamide (u.a. Sulfamethizol, Sulfadiazin, Sulfamethoxazol). Sie wiesen in vier Proben Sulfamethoxazol in Konzentrationen von 30 bis $85 \mathrm{ng} \mathrm{l}^{-1}$ nach. In drei Proben von Berliner Kläranlagenabflüssen ergab die Analyse Gehalte zwischen 5 und $240 \mathrm{ng}^{-1}$ für die oben genannten Substanzen.

Nicht nur in Oberflächengewässern und Kläranlagen, sondern auch im Grundwasser sind Sulfonamide nachweisbar, was im Gegensatz zu den Ergebnissen von Höper et al. (2002) steht: bei Lindsey et al. (2001) enthielt eine von sechs Grundwasserproben das Sulfonamid Sulfamethoxazol. Ebenso lagen sogar Tetrazykline in neun von 138 Oberflächengewässerproben vor; die Konzentrationen betrugen 0,07 bis $1,34 \mu \mathrm{g} 1^{-1}$ Oxytetrazyklin, $0,11 \mu \mathrm{g} \mathrm{l^{-1 }}$ Tetrazyklin bzw. 0,15 $\mu \mathrm{gl}^{-1}$ Chlortetrazyklin. Die Autoren vermuteten aber, dass trotz des positiven Grundwasserbefundes für Tetrazyklin ein run-off der Substanzen in die Oberflächengewässer wahrscheinlicher ist als ein Eintrag ins Grundwasser durch Auswaschung der Böden. Zhu et al. (2001) bestätigten durch ihre Ergebnisse diese Vermutung: in Grundwasserproben von elf Orten in Nebraska im Abflussbereich von Gülle-Lagunen konnten keine Tetrazykline oberhalb der Nachweisgrenze von 0,21 bis $0,28 \mu \mathrm{g} \mathrm{l}^{-1}$ nachgewiesen werden.

Im Widerspruch zu der run-off-These von Zhu et al. (2001) und Lindsey et al. (2001) steht die Arbeit von Campagnolo et al. (2002), in der besonders hohe Antibiotikamengen in Boden und auch in Grundwasser in der Nähe von so genannten Güllelagunen festgestellt werden konnten. Hier enthielten $31 \%$ der Wasserproben, die in der Nähe von Schweineställen gezogen wurden, und $67 \%$ der aus der näheren Umgebung von Hühnerställen stammenden Wasserproben antibiotische Wirkstoffe. Diese Ergebnisse deuten entgegen der Vermutung von Lindsey et al. (2001) doch auf einen Eintrag der Antibiotika durch Auswaschung hin, allerdings muß bei der Bewertung der Ergebnisse die unmittelbare Nähe zu den Eintragsquellen beachtet werden

Keine der bisher vorliegenden Untersuchungen kann allerdings mit Sicherheit ausschließen, dass Tierarzneimittel und hier im Besonderen die Antibiotika nicht im Boden verlagert werden und daher nicht ins Grundwasser gelangen. 
Den Einfluss von über Wirtschaftsdünger in den Boden eingebrachten Antibiotika auf die Bodenfauna untersuchten Baguer et al. (2000). Sie überprüften den Effekt von Tylosin und Oxytetrazyklin auf Springschwänze, Regenwürmer und Enchytraeen. Erst ab einer Konzentration von $3000 \mathrm{mg} \mathrm{kg}^{-1}$ trat überhaupt ein messbarer Effekt auf. In vielen Fällen war bis zur höchsten Testkonzentration von $5000 \mathrm{mg} \mathrm{kg}^{-1}$ kein Effekt auf die Bodenorganismen $\mathrm{zu}$ sehen. Ein Effekt durch Antibiotika auf Bodenorganismen in praxisüblichen Konzentrationen ist daher sehr unwahrscheinlich.

Im Zusammenhang mit den oben genannten Untersuchungen zum Vorkommen von Antibiotika im Boden beschäftigen sich neuere Studien verstärkt mit der mögichen Resistenzausbildung bei Bodenbakterien (wie z.B. Pseudomonas spp., Bacillus cereus) gegenüber den Antibiotika. Eine ausführliche Darstellung würde den Rahmen der vorliegenden Arbeit sprengen. Beispielhaft soll die folgende erwähnt werden: Sengeløv et al. (2003) untersuchten Böden, die mit tetrazyklinbelasteter Schweinegülle (TetrazyklinKonzentrationen der Gülle variierten von 42 bis $698 \mu \mathrm{g} \mathrm{l}^{-1}$ ) versetzt worden waren, auf das Vorkommen tetrazyklinresistenter Bakterien. Die mit der Gülle beaufschlagten Böden enthielten schließlich Tetrazyklin in geschätzten Konzentrationen von 0,6, 1,3 und 14,5 $\mu \mathrm{g} \mathrm{kg}^{-1}$. Der Boden mit der höchsten TC-Konzentration erbrachte jeweils ein verstärktes Auftreten der resistenten Bakterien. Nach ca. fünf Monaten nahm die Tetrazyklinresistenz in allen Böden deutlich ab und entsprach dann mengenmäßig den Kontrollböden.

\subsection{Vorkommen von Antibiotika in Pflanzen}

Schließlich ist ein weiterer wichtiger Aspekt zu beachten: wenn Tierarzneimittel im Boden vorkommen, dort durch unterschiedliche Mechanismen festgelegt oder aber auch ins Wasser verlagert werden können, wie wahrscheinlich ist die Aufnahme derselben durch Pflanzen.

McCoy (1976) führte schon früh Untersuchungen durch, um diese Frage zu klären. Er testete die Aufnahme von Oxytetrazyklin durch Kokospalmen und konnte nur bei direkter Injektion von in Wasser gelöstem Oxytetrazyklin in den Stamm Spuren des Antibiotikums in Wurzeln, Stamm und Früchten feststellen. Eine Gabe des Antibiotikums in Form von Vernebelung oder Bewässerung ergab keine Aufnahme in die Pflanze. Ebenso konnte McCoy auch keinerlei Aufnahme bei Pintobohnen feststellen, was durch Batchelder (1981) bestätigt wurde. In weiteren Versuchen wies Batchelder (1982) eine Ertragszunahme bei Radieschen, Weizen und Mais durch Zugabe von $160 \mathrm{mg} \mathrm{kg}^{-1}$ Tetrazyklin nach. 
Demgegenüber war das Wachstum von Pintobohnen signifikant reduziert, allerdings nur in einem sandigen, nicht aber in einem tonigen Lehm. Der Autor erklärte diesen Effekt mit einer Hemmung der Rhizobien bzw. der Wurzelnodulation und vermutete, dass auch andere Leguminosen möglicherweise sensitiv reagieren

Migliore et al. (1995) testeten den Effekt von $300 \mathrm{mg}^{1^{-1}}$ Sulfadimethoxin (verabreicht über eine Nährlösung) u.a. auf Mais und ermittelten eine Verminderung im Wurzel-, Blatt- und Stengelwachstum gegenüber den Kontrollpflanzen ohne Antibiotikum.Die Autoren führen dies auf die Bioakkumulation des Wirkstoffes in der Pflanze zurück.

Jjemba (2002) kritisiert die teilweise überhöhten und damit nicht praxisrelevanten Konzentrationen, mit denen in den wenigen $\mathrm{zu}$ dem Thema vorliegenden Versuchen getestet wurde. Insgesamt ist bis heute nicht abschließend geklärt, ob eine Gefahrvon über Pflanzen aufgenommener Antibiotika ausgeht. 


\section{Material und Methoden}

\subsection{Entwicklung von Nachweismethoden}

Grundlage für die experimentellen Untersuchungen bildete die Methodenentwicklung zum Nachweis von Tetrazyklinen und Sulfonamiden in den $\mathrm{zu}$ untersuchenden Matrizes Masthähnchenfestmist, Sickerwasser und Schweinegülle. Da die Ausscheidungen normalerweise als Gülle (ein Kot-Harn-Gemisch) oder vermischt mit Einstreu als Festmist vorliegen, war eine differenzierte Betrachtung der Ausscheidungen über Kot oder über Harn im vorliegenden Fall nicht notwendig. Als geeignetes Nachweisverfahren hat sich hier die Hochleistungsflüssigchromatographie (HPLC), gekoppelt mit einer UV-Detektion, erwiesen. Die Methode hat ihren Ursprung in der Lebensmittelanalytik (BGVV 1995; Staatliches Lebensmitteluntersuchungsamt Oldenburg 1999). Mittlerweile ist die Analytik um die Kopplung der HPLC mit dem Massenspektrometer (MS) erweitert worden, wie verschiedene Studien zeigen (Hartig et al. 1999; Hamscher et al. 2000; Lindsey et al. 2001; Haller et al. 2002).

Erste Arbeiten von Grafe (2001) zum Nachweis von Tetrazyklin in Schweinegülledienten als Grundlage der im Folgenden beschriebenen Nachweismethoden. Änderungen betrafen vornehmlich die Probenaufbereitung, die von Matrix zu Matrix unterschiedlich ist. Die Zusammensetzung und Konfiguration der HPLC-Apparatur konnte, soweit nicht in den folgenden Kapiteln extra erwähnt, für alle Nachweismethoden angewendet werden und ist daher exemplarisch an dieser Stelle aufgelistet. Alle eingesetzten Fließmittel hatten HPLCQualität (gradient grade $=$ gr. gr.).

\section{HPLC-Konfiguration:}

Merck-Hitachi HPLC-System: Pumpe L-7100

Autosampler L-7200

Solvent-Degasser L-7612

Interface D-7000

Säulenofen L-7360 $\left(25^{\circ} \mathrm{C}\right)$

Säule: Lichrospher 60 Select B 250-4 RP-18 (5 $\mu \mathrm{m})$

Vorsäule: Lichrospher 100 RP-18 $(5 \mu \mathrm{m})$

Flow: $0,8 \mathrm{ml} / \mathrm{min}$

Injektionsmenge: $20 \mu 1$

UV-VIS-Detektor L-4250 
PC-Software D-7000 HPLC System Manager

Mobile Phase:

Solvent A: $20 \%$ Oxalsäure 0,01 M : $80 \%$ Acetonitril

Solvent B: Acetonitril gr. gr.

Die Messwellenlänge des Detektors betrug bei Tetrazyklinen $350 \mathrm{~nm}$ und bei Sulfonamiden $285 \mathrm{~nm}$. Beide Einstellungen stellen einen Kompromiss dar, da jede Substanz eigene Optima in der Wellenlänge zeigt. Um aber in jeweils einer Messung alle Tetrazykline bzw. alle Sulfonamide analysieren zu können, fiel die Entscheidung zugunsten dieser beiden Wellenlängen.

\subsubsection{Tetrazyklin-HCl in Masthähnchenfestmist}

Frischer, unbearbeiteter Masthähnchenfestmist besteht zu veränderlichen Gewichtsanteilen aus Kot und Stroh und weist damit neben einer stark inhomogenen Struktur eine hohe Variabilität in Nährstoff- und Trockensubstanzgehalt auf. Eine Vielzahl von organischen Substanzen und unterschiedliche Gehalte an zwei und dreiwertigen Kationen (Kalzium, Magnesium, Eisen etc.) erschweren den Nachweis der Tetrazykline, da diese wie schon in Kapitel 2.2 erwähnt zum Teil irreversible Chelatkomplexe mit diesen Stoffen eingehen. Darüber hinaus erfolgt durch die Zugabe von Stroh als Einstral eine Verdünnung der Substanz, obwohl Tetrazyklin aufgrund der gebildeten Chelate mit den in den Faeces enthaltenen Kationen weiterhin eine stärkere Bindung zu den Kotbestandteilen als zur Kohlenstofffraktion aufweist.

Da Tetrazykline bei hohen Temperaturen instabil sind (Kühne et al. 2001), war eine gewöhnliche Trocknung des Probenmaterials bei $105^{\circ} \mathrm{C}$ nicht möglich. Es wurde daher eine Gefriertrocknung durchgeführt und der Mist im Anschluss fein vermahlen (Brabender, 1,5 mm). Durch diese Vorgehensweise konnte ein homogenes Substrat unter größtmöglicher Schonung des Wirkstoffes Tetrazyklin erzielt werden. Die Nachweisgrenze beträgt $1 \mathrm{mg} \mathrm{kg}^{-1}$ im Ausgangsmaterial.

\section{Verwendete Chemikalien:}

Zitronensäure-Zitrat-Puffer (pH 4,8), 1 M

Tetrazyklin-HCl (Sigma, Art. Nr. T-3383)

4-Epi-Tetrazyklin-HCl (Acros Organics, Art. Nr. 23312) 


\section{Probenaufbereitung:}

Die frischen Masthähnchenfestmistproben wurden über einen Zeitraum von 24 Stunden bei einer maximalen Stellflächentemperatur von $20^{\circ} \mathrm{C}$ im Gefriertrockner (Martin Christ LMC-1, Gamma 1-20) getrocknet und anschließend fein gemahlen. $2 \mathrm{~g}$ des auf diese Weise homogenisierten Materials wurden mit Zitratpuffer $(\mathrm{pH} \mathrm{4,8)}$ versetzt $(1: 20 \mathrm{~m} / \mathrm{m})$. Nach Extraktion im Horizontalschüttler (Gerhardt; Programm 100) über 30min wurde das Material in der Zentrifuge (Minifuge T; Heraeus Sepatech) bei $6198 \mathrm{~g}$ (10 min) in Festund Flüssigphase getrennt. Die Flüssigphase wurde filtriert (Faltenfilter; Schleicher \& Schüll, Ø $150 \mathrm{~mm}, 595 \frac{1}{2}$ ). Das Filtrat wurde unter Nutzung von MicrospinZentrifugenfiltern (Roth, Volumen $<2 \mathrm{ml}$ ) bei $2754 \mathrm{~g}$ für $20 \mathrm{~min}$ zentrifugiert. Die so erhaltene klare Lösung konnte nach der Überführung in Autosamplerfläschchen direkt der HPLC-Messung zugeführt werden.

\section{Analyse:}

Die Gradienteneinstellung, die bei einer Laufzeit von 15 min vorgenommen wurde, ist in der nachfolgenden Tabelle 4 wiedergegeben.

Tabelle 4: Gradientenprogramm für den Tetrazyklinnachweis in Masthähnchenfestmist

\begin{tabular}{lcc}
\hline Laufzeit & $\begin{array}{r}\text { Solvent A: 20 \% Oxalsäure 0,01 M } \\
+\mathbf{8 0} \% \text { Acetonitril }\end{array}$ & $\begin{array}{c}\text { Solvent B: } \\
\text { Acetonitril } \\
(\mathbf{m i n})\end{array}$ \\
\hline 0,0 & $\mathbf{( \% )}$ & $\mathbf{( \% )}$ \\
2,5 & 100,0 & 0,0 \\
10,0 & 95,0 & 5,0 \\
$13,5-15,0$ & 65,0 & 35,0 \\
\hline
\end{tabular}

Zur Kalibrierung wurden je Messtermin externe Standards für Tetrazyklin und 4EpiTetrazyklin in Konzentrationen von 1 bis $100 \mathrm{mg} \mathrm{kg}^{-1}$ in wässriger Lösung hergestellt. Je Messtermin wurde mindestens eine Leerprobe (Masthähnchenfestmist ohne Tetrazyklin, in diesem Versuch dienten die Kontrollen diesem Zweck) mitgemessen, um mögliche Verunreinigungen der Probe und Störungen im System erkennen zu können.

\section{Wiederfindung:}

Um die Wiederfindungsrate für die Nachweismethode zu ermitteln, wurde tetrazyklinfreier Festmist in frischem Zustand mit Tetrazyklin in Konzentrationen von 5 bis $10 \mathrm{mg} \mathrm{kg}^{-1}$ 
dotiert. Im Anschluss wurden die dotierten Proben analog oben beschriebener Methode analysiert. Die mittlere Wiederfindungsrate betrug 94,9\% (Tabelle 5). Um diesen Wert wurden die späteren Messergebnisse korrigiert.

Tabelle 5: Wiederfindungsrate (\%) von Tetrazyklin (TC) einschließlich 4-EpiTetrazyklin in Masthähnchenfestmist (Frischmist)

\begin{tabular}{lcc}
\hline Hähnchenfestmistprobe & \multicolumn{1}{c}{ Wiederfindungsrate (\%) bei zwei } & TC-Konzentration \\
\cline { 2 - 3 } & $\mathbf{5} \mathbf{~ m g ~ k g}^{-\mathbf{1}}$ & $\mathbf{1 0} \mathbf{~ m g ~ k g ~}^{-\mathbf{1}}$ \\
\hline 1 & 87,1 & 72,5 \\
2 & 83,9 & 87,9 \\
3 & 110,1 & 105,6 \\
4 & 108,9 & 103,1 \\
\hline Mittelwert & 97,5 & 92,3 \\
Standardabweichung & 12,1 & 13,3 \\
\hline
\end{tabular}

\subsubsection{Ausgewählte Tetrazykline und Sulfonamide in Schweinegülle}

Die Methode von Grafe (2001) wurde um den Nachweis von Oxytetrazyklin, Chlortetrazyklin, Sulfadiazin und Sulfamethazin erweitert. Mit Ausnahme von Sulfadiazin können alle Hauptmetaboliten (Epimer-Formen der Tetrazykline, N4-AcetylSulfamethazin) mitbestimmt werden. Der Hauptmetabolit des Sulfadiazins (N4-AcetylSulfadiazin) ist nicht im Handel erhältlich, so dass nur ein qualitativer Nachweis erfolgen konnte.

Wie Masthähnchenfestmist weist auch Gülle eine hohe Variabilität in Trockensubstanzund Nährstoffgehalten auf und bildet aufgrund einer Vielzahl organischer Bestandteile im Gegensatz $\mathrm{zu}$ rein wässrigen Lösungen eine komplizierte Matrix. Eine sorgfältige Homogenisierung schon bei der Probennahme, aber auch in allen weiteren Aufbereitungsschritten, ist von großer Bedeutung. Dies wird aus den Ergebnissen einer Voruntersuchung deutlich, bei der sowohl die Mischphase (Ø TS-Gehalt 5,5\%) als auch Flüssig- und Festphase (Ø TS-Gehalt 1,9 bzw. 17,8 \%) nach Zentrifugation bei $6198 \mathrm{~g}$ analysiert wurden (Abbildung 4). Die Mischphase enthielt die Antibiotika in folgenden, zuvor mit unten aufgeführter Methode gemessenen Konzentrationen: Tetrazyklin $8,6 \mathrm{mg} \mathrm{kg}^{-1}$; Chlortetrazyklin $63,0 \mathrm{mg} \mathrm{kg}^{-1}$; Sulfadiazin $58,0 \mathrm{mg} \mathrm{kg}^{-1}$; Sulfamethazin $14,4 \mathrm{mg} \mathrm{kg}^{-1}$. 


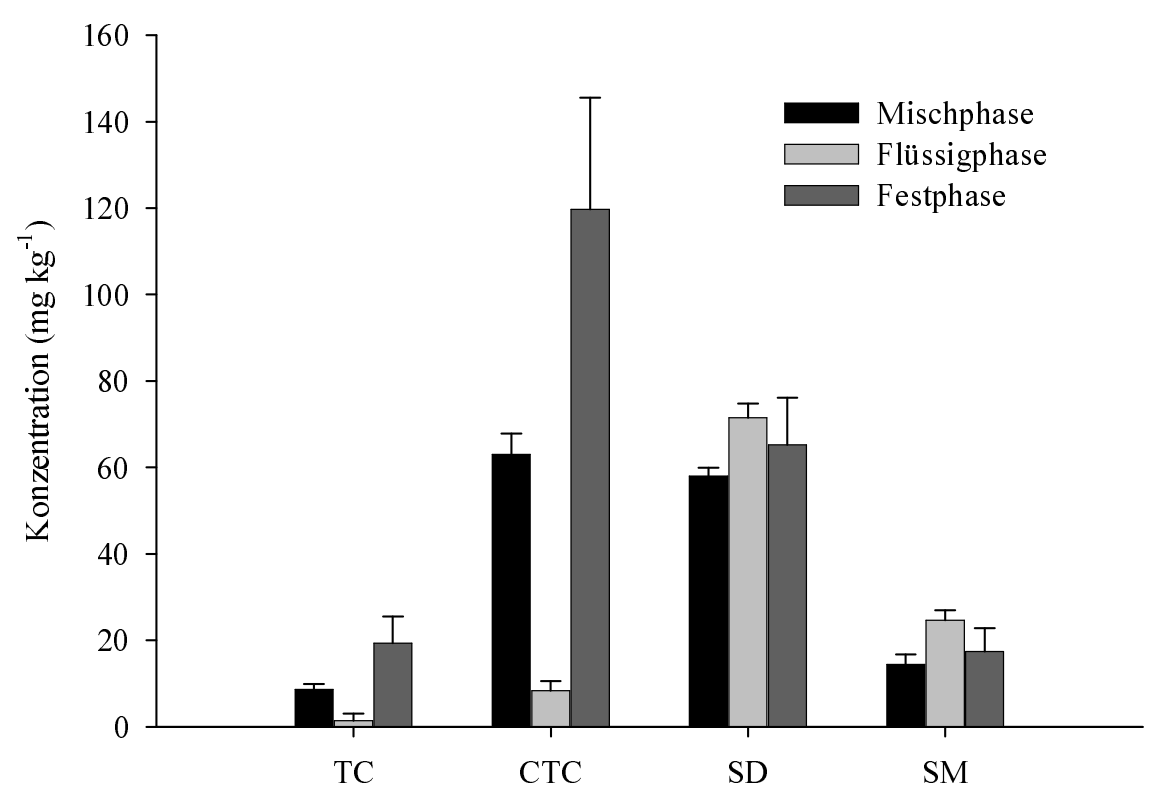

Abbildung 4: Verteilung ausgewählter Antibiotika(Tetrazyklin (TC), Chlortetrazyklin (CTC), Sulfadiazin (SD), Sulfamethazin (SM)) in der Fest-, Misch- und Flüssigphase einer Schweinegülle (mit Standardabweichung T, Mittelwert aus vier Wiederholungen)

Bei Tetrazyklin und Chlortetrazyklin befindet sich nach dem Zentrifugieren deutlich mehr Wirkstoff in der festen Phase, bei den Sulfonamiden ist dieser Effekt nicht ausgeprägt. Nicht nur den Homogenisierungsschritten in der Probenaufbereitung, sondern auch dem Aufrühren der Gülle im Güllebehälter bei der Probenentnahme ist aufgrund dieser Besonderheit größte Beachtung $\mathrm{zu}$ geben. Die Bestimmungsgrenze der unten beschriebenen Methode beträgt für Tetrazyklin, Chlortetrazyklin, Sulfadiazin und Sulfamethazin $0,8 \mathrm{mg} \mathrm{kg}^{-1}$, für Oxytetrazyklin $1,0 \mathrm{mg} \mathrm{kg}^{-1}$.

\section{Verwendete Chemikalien:}

Zitronensäure-Zitrat-Puffer (pH 4,8), $1 \mathrm{M}$

Tetrazyklin-HCl (Sigma, Art. Nr. T-3383)

4-Epi-Tetrazyklin-HCl (Acros Organics, Art. Nr. 23312)

Chlortetrazyklin-HCl (Sigma, Art. C-4881)

Epi-Chlortetrazyklin-HCl (Acros Organics Lot AO 14963301)

Oxytetrazyklin-HCl (ICN Biomedicals Inc Lot-Nr. 5883C)

Epi-Oxytetrazyklin (Acros Organics Lot AO 14381601)

Sulfadiazin Natrium-Salz (Sigma, Art. Nr. S-3549) 
Sulfamethazin Natrium-Salz (Sigma, Art. Nr. S-5637)

N4-Acetyl-Sulfamethazin (ICN Biomedicals Inc Lot-Nr. 105032)

\section{Probenaufbereitung:}

Die bei $-18^{\circ} \mathrm{C}$ gelagerte Gülle wurde im Kühlschrank $\left(4^{\circ} \mathrm{C}\right)$ aufgetaut. Nach Homogenisierung der Gülle mit dem Ultra-Turrax (T 25 mit Dispergierstab S 25 N-25 F, IKA Labortechnik, $20.000 \mathrm{U} \mathrm{min}^{-1}$ für $1 \mathrm{~min}$ ) wurden $2 \mathrm{~g}$ der Gülle mit $38 \mathrm{ml}$ Zitratpuffer (pH 4,8) versetzt (1:20). Das Gemisch wurde zentrifugiert (Minifuge T; Heraeus Sepatech, $10 \mathrm{~min}$ bei $6198 \mathrm{~g}$ ) und der Überstand filtriert (Schleicher \& Schüll, Ø $150 \mathrm{~mm}, 595$ 1⁄2). Nach einer weiteren Zentrifugation (2754 g, $20 \mathrm{~min}$ ) mittels Microspin-Zentrifugenfiltern (Roth, Volumen $<2 \mathrm{ml}$ ) konnte die so gewonnene klare Lösung direkt der HPLC zugeführt werden.

\section{Analyse:}

Die in Tabelle 6 aufgeführte Gradienteneinstellung ist bei einer Laufzeit von $15 \mathrm{~min} \mathrm{zu}$ beachten. Zur Kalibrierung wurden je Messtermin externe Standards in Konzentrationen von 0,1 bis $10 \mathrm{mg} \mathrm{kg}^{-1}$ für alle Komponenten in wässriger Lösung hergestellt.

Tabelle 6: Gradientenprogramm für den Tetrazyklin- und Sulfonamidnachweis in Schweinegülle

\begin{tabular}{lcc}
\hline Laufzeit & $\begin{array}{c}\text { Solvent A: 20 \% Oxalsäure 0,01 M } \\
+\mathbf{8 0} \% \text { Acetonitril }\end{array}$ & $\begin{array}{c}\text { Solvent B: } \\
\text { Acetonitril } \\
(\mathbf{m i n})\end{array}$ \\
\hline 0,0 & $\mathbf{( \% )}$ & $\mathbf{( \% )}$ \\
7,0 & 100,0 & 0,0 \\
12,0 & 63,0 & 37,0 \\
$13,0-15,0$ & 63,0 & 37,0 \\
\hline
\end{tabular}

\section{Wiederfindung:}

Die Wiederfindungsraten für die einzelnen Antibiotika wurden mit dotierter Schweinegülle in verschiedenen Konzentrationsstufen ermittelt. $\mathrm{Zu}$ diesem Zweck wurden fünf unterschiedliche Schweinegüllen mit den fünf ausgewählten Antibiotika Chlortetrazyklin, Oxytetrazyklin und Tetrazyklin, Sulfadiazin und Sulfamethazin in vier verschiedenen Konzentrationen $\left(0,8 / 1,0 / 2,0\right.$ und $\left.8,0 \mathrm{mg} \mathrm{kg}^{-1}\right)$ dotiert (Wirkstoffe gelöst in destilliertem Wasser, Vermischung über Horizontalschüttler Gerhardt, Programm 100), anschließend homogenisiert und aufbereitet. Die aufbereitete Injektionslösung wurde in fünf Aliquote 
geteilt. Die Analyse der Proben erfolgte an fünf nicht unmittelbar aufeinander folgenden Tagen (Tag 0, 2, 23, 29, 30). Zwischen den Messungen wurden die Proben dunkel und kühl $\left(4{ }^{\circ} \mathrm{C}\right)$ gelagert, um einen möglichen $\mathrm{Ab}$ - bzw. Umbau der Wirkstoffe zu vermeiden.

Der statistischen Auswertung mittels Varianzanalyse (GLM) lagen folgende Modelle zugrunde:

\section{1) innerhalb der einzelnen Konzentrationsstufen (Modell 1):}

$$
\mathrm{y}=\mu+\mathrm{g}_{\mathrm{i}}+\mathrm{t}_{\mathrm{j}}+\mathrm{a}_{\mathrm{k}}+\mathrm{ga}_{\mathrm{ij}}+\mathrm{ta}_{\mathrm{jk}}+\mathrm{e}
$$

wobei

$\mathrm{y} \quad=$ der beobachtete Prüfgliedmittelwert

$\mu \quad=$ der allgemeine Mittelwert

$\mathrm{g}_{\mathrm{i}} \quad=$ der Effekt der i-ten Gülle (Gülle 1-5)

$\mathrm{t}_{\mathrm{j}} \quad=$ der Effekt des j-ten Tages (Tag 0, 2, 23, 29, 30)

$\mathrm{a}_{\mathrm{k}} \quad=$ der Effekt des k-ten Antibiotikums (TC, OTC, CTC, SD, SM)

ga $_{\text {ik }}=$ der Effekt der Wechselwirkung der i-ten Gülle mit dem k-ten Antibiotikum

$\mathrm{ta}_{\mathrm{jk}}=$ der Effekt der Wechselwirkung des j-ten Tages mit dem k-ten Antibiotikum

e $\quad=$ Restfehler

ist.

\section{2) innerhalb der Wirkstoffe (Modell 2):}

$$
\mathrm{y}=\mu+\mathrm{g}_{\mathrm{i}}+\mathrm{t}_{\mathrm{j}}+\mathrm{k}_{\mathrm{k}}+\mathrm{tk}_{\mathrm{jk}}+\mathrm{gk}_{\mathrm{ik}}+\mathrm{e}
$$

wobei

$\mathrm{y}=$ der beobachtete Prüfgliedmittelwert

$\mu \quad=$ der allgemeine Mittelwert

$\mathrm{g}_{\mathrm{i}} \quad=$ der Effekt der i-ten Gülle (Gülle 1-5)

$\mathrm{t}_{\mathrm{j}} \quad=$ der Effekt des j-ten Tages (Tag 0, 2, 23, 29, 30)

$\mathrm{k}_{\mathrm{k}} \quad=$ der Effekt der k-ten Konzentrationsstufe $\left(0,8 / 1,0 / 2,0 / 8,0 \mathrm{mg} \mathrm{kg}^{-1}\right)$

$\mathrm{tk}_{\mathrm{jk}}=$ der Effekt der Wechselwirkung des j-ten Tages mit der k-ten Konzentrationsstufe

$\mathrm{gk}_{\mathrm{ik}}=$ der Effekt der Wechselwirkung der i-ten Gülle mit der k-ten Konzentrationsstufe

e $\quad=$ Restfehler

ist.

Auf die jeweiligen Konzentrationsstufen bezogen ergab das Modell 1, dass lediglich bei einer Konzentration von $0,8 \mathrm{mg} \mathrm{kg}^{-1}$ ein signifikanter Einfluss der verwendeten Güllen bestand $(\mathrm{p} \leq 0,05)$. Der Einfluss des Messtags war mit Ausnahme der Konzentrationsstufe $1,0 \mathrm{mg} \mathrm{kg}^{-1}$ signifikant. 
Ein Abbau der Antibiotika als Erklärung für den möglichen Einfluss des Messtages über die fünf Analysetage ist anhand der Ergebnisse nicht erkennbar. Tabelle 7 und Tabelle 8 zeigen die p-Werte des jeweiligen Signifikanzniveaus und $\mathrm{r}^{2}$ für Modell 1 und 2. Entsprechend der Signifikanzniveaus von $\mathrm{p} \leq 0,05, \mathrm{p} \leq 0,01$ und $\mathrm{p} \leq 0,001$ wurden ein bis drei Sternchen vergeben.

Tabelle 7: Ergebnisse der Varianzanalyse mit den Signifikanzniveaus und Freiheitsgraden (df) innerhalb der Konzentrationsstufen (Konz I - IV) der untersuchten Antibiotika in Schweinegülle (Modell 1)

\begin{tabular}{|c|c|c|c|c|c|}
\hline \multirow[t]{3}{*}{ Parameter } & \multicolumn{2}{|l|}{ Freiheitsgrade } & \multicolumn{3}{|c|}{ Wirkstoffkonzentration $\left(\mathrm{mg} \mathrm{kg}^{-1}\right)$} \\
\hline & \multirow[t]{2}{*}{ df } & Konz I & \multirow{2}{*}{$\begin{array}{r}\text { Konz II } \\
1,0\end{array}$} & Konz III & \multirow{2}{*}{$\begin{array}{r}\text { Konz IV } \\
8,0\end{array}$} \\
\hline & & 0,8 & & 2,0 & \\
\hline Gülle (G) & 4 & $*$ & 0,860 & 0,159 & 0,955 \\
\hline Tag (d) & 4 & $* *$ & 0,190 & * & ** \\
\hline $\begin{array}{l}\text { Antibiotika } \\
\text { (A) }\end{array}$ & $3^{1 / 4}$ & $* * *$ & $* * *$ & $* * *$ & $* * *$ \\
\hline $\mathrm{G} \times \mathrm{A}$ & $12^{1} / 16$ & 0,406 & 0,075 & $* *$ & 0,593 \\
\hline $\mathrm{d} \times \mathrm{A}$ & $12^{1} / 16$ & $* *$ & * & $* *$ & * \\
\hline Fehler & $61^{1 / / 79^{2} / 80}$ & & & & \\
\hline Gesamt & $97^{1} / 124^{2} / 125$ & & & & \\
\hline \multicolumn{6}{|c|}{$\mathrm{p} \leq 0,05=*, \mathrm{p} \leq 0,01=* *, \mathrm{p} \leq 0,001=* * *$} \\
\hline Konz I & \multicolumn{5}{|c|}{$\mathrm{r}^{2}=0,763$ (korrigiertes $\left.\mathrm{r}^{2}=0,627\right)$} \\
\hline Konz II & \multicolumn{5}{|c|}{$\mathrm{r}^{2}=0,847$ (korrigiertes $\mathrm{r}^{2}=0,762$ ) } \\
\hline Konz III & \multicolumn{5}{|c|}{$r^{2}=0,964\left(\right.$ korrigiertes $\left.r^{2}=0,944\right)$} \\
\hline Konz IV & \multicolumn{5}{|c|}{$r^{2}=0,971\left(\right.$ korrigiertes $\left.r^{2}=0,955\right)$} \\
\hline $1=$ nur bei Kon & & & & & \\
\hline${ }^{2}=$ nur bei Kon & & & & & \\
\hline
\end{tabular}

Bei getrennter Betrachtung der einzelnen Wirkstoffe (Modell 2) ergab sich ein signifikanter Einfluss des Messtags für Tetrazyklin $(p \leq 0,001)$, Sulfadiazin $(p \leq 0,05)$ und Sulfamethazin $(\mathrm{p} \leq 0,01)$. Die verwendete Gülle beeinflusste das Messergebnis lediglich im Fall von Sulfadiazin $(\mathrm{p} \leq 0,001)$.

Insgesamt wird aus dieser Analyse deutlich, dass die Messwerte keinen gerichteten Effekt in Bezug auf Messtageseinfluss oder Gülle aufweisen, die einzelnen Antibiotika jedoch unterschiedlich in der Analytik reagieren. 
Tabelle 8: Ergebnisse der Varianzanalyse mit den Signifikanzniveaus und Freiheitsgraden (df) innerhalb der untersuchten Antibiotika Tetrazyklin (TC), Oxytetrazyklin (OTC), Chlortetrazyklin (CTC), Sulfadiazin (SD) und Sulfamethazin $(\mathrm{SM})$ in Schweinegülle $(\mathrm{G}=$ Gülle, $\mathrm{d}=\mathrm{Tag}, \mathrm{K}=$ Konzentration) (Modell 2)

\begin{tabular}{|c|c|c|c|c|c|c|}
\hline \multirow[t]{2}{*}{ Parameter } & \multicolumn{3}{|l|}{ Freiheitsgrade } & \multicolumn{3}{|c|}{ Antibiotika } \\
\hline & df & TC & OTC & CTC & SD & SM \\
\hline $\mathrm{G}$ & 4 & 0,192 & 0,068 & 0,282 & $* * *$ & 0,271 \\
\hline d & 4 & $* * *$ & 0,163 & 0,058 & $*$ & $* *$ \\
\hline K & $3 / 2^{1}$ & $* * *$ & $* * *$ & $* * *$ & $* * *$ & $* * *$ \\
\hline $\mathrm{d} \times \mathrm{K}$ & $12 / 8^{1}$ & $*$ & 0,101 & 0,306 & 0,291 & 0,273 \\
\hline $\mathrm{G} \times \mathrm{K}$ & $12 / 8^{1}$ & 0,254 & 0,051 & 0,211 & 0,195 & 0,272 \\
\hline Fehler & \multicolumn{6}{|l|}{$64 / 48^{1} / 61^{2} / 63^{3}$} \\
\hline Gesamt & \multicolumn{6}{|l|}{$100 / 75^{1} / 97^{2} / 99^{3}$} \\
\hline $\begin{array}{l}\mathrm{p} \leq 0,05=*, \mathrm{p} \leq \\
\text { TC } \\
\text { OTC } \\
\text { CTC } \\
\text { SD } \\
\text { SM } \\
1^{1}=\text { nur für OTC } \\
{ }^{2}=\text { nur für SD } \\
3^{3}=\text { nur für SM }\end{array}$ & $\begin{array}{l}0,01=* *, p \leq 0,001= \\
r^{2}=0,669 \text { (korrigierte } \\
r^{2}=0,780 \text { (korrigierte } \\
\mathrm{r}^{2}=0,760 \text { (korrigierte } \\
\mathrm{r}^{2}=0,761 \text { (korrigierte } \\
\mathrm{r}^{2}=0,584 \text { (korrigiertes }\end{array}$ & $\begin{array}{l}0,489) \\
0,661) \\
0,629) \\
0,624) \\
0,352)\end{array}$ & & & & \\
\hline
\end{tabular}

Durch den Vergleich der Mittelwerte der signifikant unterschiedlichen Konzentrationsstufen (Tukey-HSD; $\mathrm{p}<0,005)$ ergaben sich gestaffelte Wiederfindungsraten in Abhängigkeit von der Konzentration (Tabelle 9). Die Messergebnisse in Kapitel 4.2 wurden um diese Wiederfindungsraten korrigiert.

Tabelle 9: Wiederfindungsraten (\%) für ausgewählte Antibiotikasubstanzen in Schweinegülle

\begin{tabular}{|c|c|c|c|c|}
\hline \multirow[t]{2}{*}{ Substanz } & \multicolumn{4}{|c|}{ Wiederfindungsrate (\%) bei vier Konzentrationen } \\
\hline & $0.8 \mathrm{mg} \mathrm{kg}^{-1}$ & $1.0 \mathrm{mg} \mathrm{kg}^{-1}$ & $2.0 \mathrm{mg} \mathrm{kg}^{-1}$ & $8.0 \mathrm{mg} \mathrm{kg}^{-1}$ \\
\hline Tetrazyklin & \multicolumn{2}{|c|}{110,7} & \multicolumn{2}{|c|}{100,7} \\
\hline Oxytetrazyklin & - & 71,2 & \multicolumn{2}{|c|}{61,4} \\
\hline Chlortetrazyklin & \multicolumn{2}{|c|}{128,2} & \multicolumn{2}{|c|}{102,0} \\
\hline Sulfadiazin & \multicolumn{2}{|c|}{114,6} & \multicolumn{2}{|c|}{103,6} \\
\hline Sulfamethazin & \multicolumn{2}{|r|}{101,0} & & 93,6 \\
\hline
\end{tabular}




\subsubsection{Tetrazyklin und Sulfadiazin in Sickerwasser}

Tetrazykline, aber auch Sulfonamide binden an verschiedene Komponenten wie Kalzium, Magnesium, Huminstoffe, die in Sickerwasser aus Böden enthalten sind. Daher ist der Nachweis dieser Antibiotika in Sickerwasser aus Böden erschwert. Es muss mit einer starken Bindung vor allem der Tetrazykline, aber auch der Sulfonamide an diese unterschiedlichen Komponenten gerechnet werden (siehe Kapitel 2.2) (Sithole \& Guy 1987; Holten Lützhøft et al. 2000; Tolls 2001; Hamscher et al. 2002).

Aufgrund der zu erwartenden geringen Konzentration der Antibiotika im Sickerwasser war eine sensitive Nachweismethode erforderlich. Um die entsprechende Nachweisgrenze beim Einsatz von HPLC zu erreichen, sind viele Aufbereitungsschritte notwendig. Jeder zusätzliche Arbeitsschritt kann $\mathrm{zu}$ Wirkstoffverlusten führen, so dass als Kontrollmöglichkeit ein geeigneter interner Standard sowohl für die Tetrazykline als auch für die Sulfonamide eingeführt wurde.

Als interner Standard kann eine Substanz verwendet werden, die aus der gleichen Wirkstoffgruppe wie die zu analysierenden Substanzen stammt. Gleichzeitig müssen die Adsorptions- und Retentionseigenschaften des internen Standards mit denen der Leitsubstanzen weitestgehend übereinstimmen Trotzdem aber sind eine gute Trennung von den Analysesubstanzen und kein zu großer Abstand al den Peaks der Leitsubstanzen nötig (Guhde 1998). Mindestens ebenso wichtig ist aber auch, dass der interne Standard nicht in der Probe vorhanden sein darf; es müssen also Substanzen verwendet werden die wenigstens im veterinärmedizinischen Bereich in Deutschland nicht zugelassen sind besser noch auch nicht im humanmedizinischen Bereich und somit nicht in der Probe zu erwarten sind. Für die Substanzklasse der Tetrazykline fiel die Wahl auf Doxyzyklin, für Sulfonamide auf Sulfaphenazol.

Um auch geringe Konzentrationen im Sickerwasser nachweisen zu können, ist die Festphasenextraktion mittels spezieller Kartuschen ein geeignetes Verfahren zur Aufkonzentrierung des Wirkstoffes (Hamscher et al. 2002). Um zu der im Folgenden beschriebenen Methode zu gelangen, waren jedoch viele Einzelparameter zu testen, da in diesem Fall auf keine schon bestehende Standardmethode zurückgegriffen werden konnte. Optimiert wurden zu diesem Zweck:

- $\quad$ der pH-Wert für den zu verwendenden Puffer (Testbereich: pH 4 bis pH 6)

- das Konditionierungsmittel für die Kartuschen und das Extraktionsmittel. 
Als optimal erwies sich ein pH-Wert um 4,8, was in der Größenordnung auch mit anderen Methoden korrespondiert (Hamscher et al. 2000). Die Extraktion ist für Tetrazykline und Sulfonamide aufgrund ihrer unterschiedlichen chemischen Eigenschaften verschieden, so dass für jede Wirkstoffgruppe eigene Extraktionslösungen gefunden werden mussten. Alle Versuche zur Methodenfindung wurden mit unbelastetem, den späteren Proben möglichst ähnlichem Sickerwasser (aus der Lysimeteranlage) durchgeführt.

\section{Konditionierung und Extraktion bei der Festphasenextraktion}

Während Methanol als Mittel zur Konditionierung der Kartuschen zur Festphasenextraktion (Macherey \& Nagel) aufgrund einer Beispielanwendung feststand, wurden verschiedene Extraktionsmittel getestet: Methanol, Acetonitril, 2\%ige Essigsäure in Methanol, methanolische Oxalsäure $(0,01 \mathrm{M})$, Aceton, Chloroform, Ethanol und Trichloressigsäure in Methanol in verschiedenen Konzentrationen $(0,05 \%, 0,1 \%, 0,5 \%$, $1,0 \%$ ) (Abbildung 5).

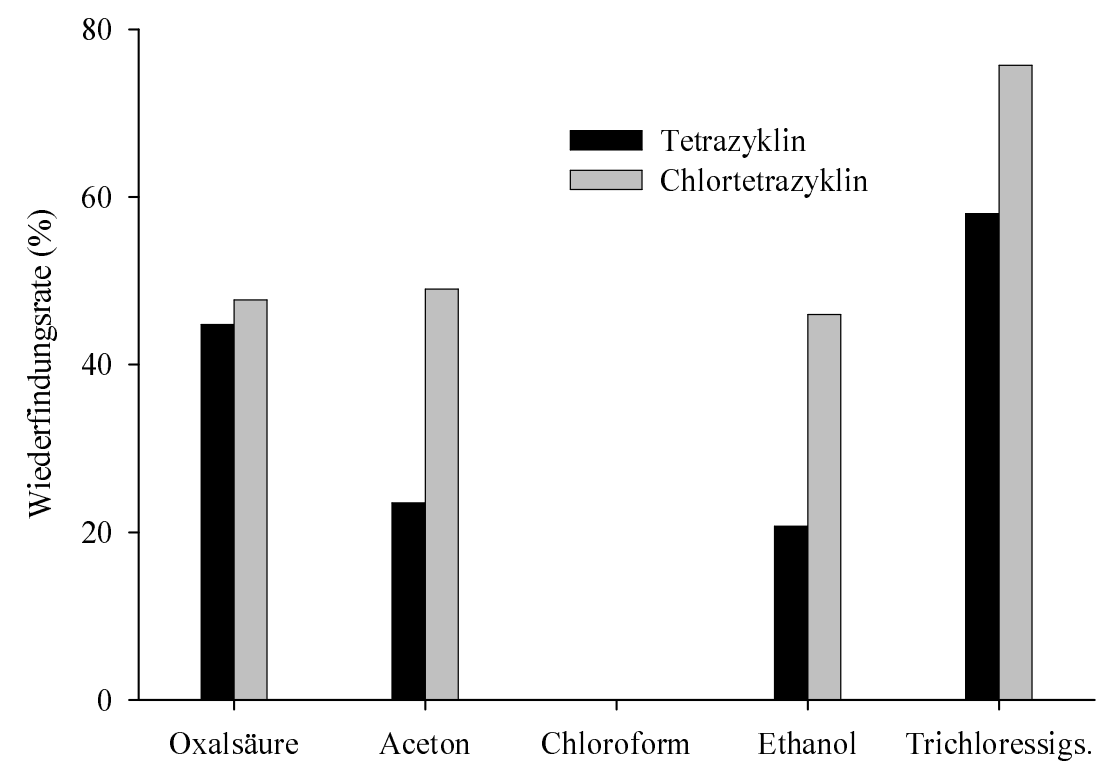

Abbildung 5: Wiederfindungsrate (\%) für Tetrazyklin und Chlortetrazyklin bei ausgewählten Extraktionsmitteln (Oxalsäure = methanolische Oxalsäure $0,01 \mathrm{M}$; Trichloressigs. $=0,1 \%$ Trichloressigsäure in Methanol) bei $\mathrm{pH}$ 4,8 (externe Kalibration, Mittelwert aus drei Messungen)

Die Variante $0,1 \%$ Trichloressigsäure in Methanol erwies sich gegenüber den anderen Extraktionsmitteln als leistungsfähigstes Extraktionsmittel. Eine Modifizierung der Anteile von Trichloressigsäure in Methanol von $0,05 \%$ bis zu 1,0 \% führte schließlich zum besten Ergebnis: 0,5 \% Trichloressigsäure in Methanol (Abbildung 6). 


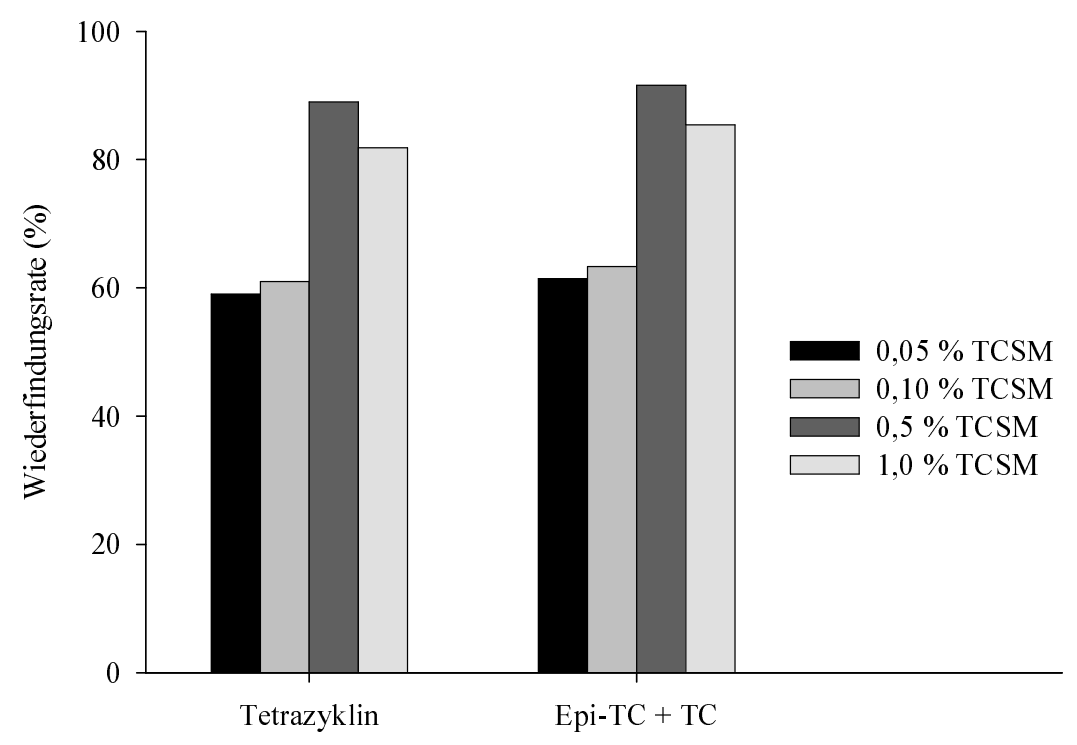

Abbildung 6: Wiederfindungsrate (\%) für Tetrazyklin und 4-Epi-Tetrazyklin (Epi-TC) + Tetrazyklin (TC) mit verschiedenen Konzentrationen des Extraktionsmittels Trichloressigsäure in Methanol(TCSM) bei pH 4,8 (externe Kalibration, Mittelwert aus drei Messungen)

Die überprüften Extraktionsmittel unterschieden sich vor allem in der Polarität. Tetrazyklin als polare Substanz kann nur mit einem ähnlich polaren Lösungsmittel wieder von der Kartuschenoberfläche extrahiert werden.

Auch das Extraktionsvolumen beeinflusste den Wiederfindungserfolg. Da mit einem Volumen von $2 \mathrm{ml}$ bereits etwa $90 \%$ des Wirkstoffes extrahiert werden konnte, wurde auf eine weitere Volumenerhöhung verzichtet. Dies hätte lediglich zu einer Verdünnung geführt und sich nachteilig auf die Bestimmungsgrenze ausgewirkt. Durch die oben beschriebene Optimierung wurde eine Bestimmungsgrenze für Chlortetrazyklin und Tetrazyklin von $1 \mu \mathrm{g}^{-1}$ erreicht.

Um eine hohe Wiederfindungsrate und eine niedrige Bestimmungsgrenze $\mathrm{zu}$ erreichen, wurde auch beim Nachweis der Sulfonamide der pH-Wert des Puffers optimiert (Testbereich: $\mathrm{pH}$ 2,5 bis $\mathrm{pH}$ 5,6). Dies geschah in Anlehnung an bereits vorliegende Methoden (Thomas et al. 1997; Ternes 2001; Pfeifer et al. 2002). Neben Methanol wurde die Kartusche probehalber mit unpolarem Hexan konditioniert, was bei Hexan zu einem sehr schlechten Adsorptionsergebnis des Sulfonamids an die Kartuschenoberfläche führte. Als Extraktionsmittel wurden methanolische Oxalsäure (0,01 M), Acetonitril, Methanol und $0,5 \%$ Trichloressigsäure in Methanol getestet. Die besten Ergebnisse wurden mit 
reinem Methanol erzielt. Die Bestimmungsgrenze liegt mit unten beschriebener Methode bei $3 \mu \mathrm{g}^{-1}$.

Für die Bestimmung der Bestimmungsgrenze in Sickerwasser sind allerdings immer die Probenmenge und die Extraktionsvolumina entscheidend, da es sich bei der Festphasenextraktion um eine Aufkonzentrierung handelt. Niedrige Wirkstoffkonzentrationen können daher bei großen Probenvolumina besser angereichert werden, da sich der Anreicherungsfaktor bei gleich bleibendem Extraktionsvolumen vergrößert.

\section{Verwendete Chemikalien:}

Zitronensäure-Zitrat-Puffer ( $\mathrm{pH} 4,8$ ), $1 \mathrm{M}$

Tetrazyklin-HCl (Sigma, Art. Nr. T-3383)

4-Epi-Tetrazyklin-HCl (Acros Organics, Art. Nr. 23312)

Chlortetrazyklin-HCl (Sigma, Art. C-4881)

Epi-Chlortetrazyklin-HCl (Acros Organics Lot AO 14963301)

Doxyzyklin-HCl (Sigma, Art. Nr. D-9891)

Sulfadiazin Natrium-Salz (Sigma, Art. Nr. S-3549)

Sulfaphenazol (Sigma, Art. Nr. S-0758)

Methanol gr. gr.

Trichloressigsäure

\section{Probenaufbereitung:}

Bis zur Festphasenextraktion wurde eine einheitliche Probenaufbereitung für den Nachweis von Tetrazyklinen und Sulfonamiden durchgeführt. Die Sickerwasserproben (Lagerung: $-18^{\circ} \mathrm{C}$ ) wurden nach dem Auftauen kräftig geschüttelt. $100 \mathrm{ml}$ Sickerwasser wurden mit Zitronensäure-Zitrat-Puffer $(\mathrm{pH} \mathrm{4,8)} \mathrm{im} \mathrm{Verhältnis} \mathrm{1:1} \mathrm{versetzt.} \mathrm{Als} \mathrm{interner}$ Standard wurde Doxyzyklin für den Tetrazyklinnachweis sowie Sulfaphenazol für den Sulfonamidnachweis verwendet. Dieser wurde in festgelegter Konzentration von $0,01 \mathrm{mg}^{-1}$ den Proben zugesetzt.

Nach Filtration (Faltenfilter, Schleicher \& Schüll, Ø $150 \mathrm{~mm}, 595 \frac{1}{2}$ ) erfolgte eine Anreicherung mittels Festphasenextraktion an Polystyrol-Divinylbenzol Adsorberharz. Zu diesem Zweck wurden die Festphasenkartuschen (Macherey \& Nagel, LV, Chromabond HR-P, Art. Nr. 732108) mit $2 \mathrm{ml}$ Methanol und $2 \mathrm{ml}$ destilliertem Wasser konditioniert. Dann erfolgte die Probenaufgabe mit einer maximalen Durchflussgeschwindigkeit von 
$4 \mathrm{ml} \mathrm{min}^{-1}$ (Regelung über Wasserstrahlpumpe). Nach einem Waschschritt $(2 \mathrm{x} 2 \mathrm{ml}$ destilliertes Wasser) trocknete die Kartusche 10 min mittels Durchsaugen von Luft, um Lösungsmittelunterschiede zu vermeiden. Die Extraktion erfolgte bei Tetrazyklinen mit $2 \mathrm{x}$

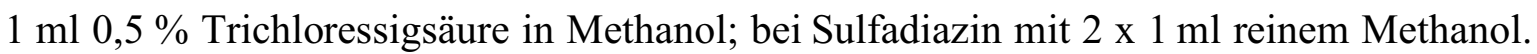

Die so gewonnene Lösung konnte direkt der HPLC-Messung zugeführt werden.

\section{Analyse:}

Die Gradienteneinstellung und die Laufzeit der Analyse sind für Tetrazykline und Sulfonamide verschieden (Tabelle 10 und Tabelle 11).

Tabelle 10: Gradientenprogramm für den Tetrazyklinnachweis in Sickerwasser bei einer Laufzeit von 15 min

\begin{tabular}{lcc}
\hline Laufzeit & $\begin{array}{c}\text { Solvent A: 20 \% Oxalsäure 0,01 M } \\
+\mathbf{8 0} \% \text { Acetonitril }\end{array}$ & $\begin{array}{c}\text { Solvent B: } \\
\text { Acetonitril }\end{array}$ \\
$(\mathbf{m i n})$ & $\mathbf{( \% )}$ & $\mathbf{( \% )}$ \\
\hline 0 & 100,0 & 0,0 \\
7,0 & 75,0 & 25,0 \\
8,0 & 70,0 & 30,0 \\
11,5 & 63,0 & 37,0 \\
$13,5-15,0$ & 100,0 & 0,0 \\
\hline
\end{tabular}

Tabelle 11: Gradientenprogramm für den Sulfadiazinnachweis in Sickerwasser bei einer Laufzeit von $17 \mathrm{~min}$

\begin{tabular}{lcc}
\hline Laufzeit & $\begin{array}{c}\text { Solvent A: 20 \% Oxalsäure 0,01 M } \\
+\mathbf{8 0} \% \text { Acetonitril } \\
(\mathbf{m i n})\end{array}$ & $\begin{array}{c}\text { Solvent B: } \\
\text { Acetonitril }\end{array}$ \\
\hline 0 & 100,0 & $\mathbf{( \% )}$ \\
8 & 63,0 & 0,0 \\
13 & 63,0 & 37,0 \\
$16-17$ & 100,0 & 37,0 \\
\hline
\end{tabular}

Zur Kalibrierung wurden je Messtermin externe Standards in Konzentrationen von 0,001 bis $0,04 \mathrm{mg}^{-1}$ für alle Komponenten in wässriger Lösung hergestellt und auch über die Festphasenextraktion angereichert. Zusätzlich wurde der interne Standard benutzt. 


\section{Wiederfindung:}

Die Wiederfindungsrate wurde für die verschiedenen Antibiotika und für unterschiedliche Konzentrationsstufen ermittelt. Antibiotikafreies Sickerwasser wurde mit Tetrazyklin und Chlortetrazyklin oder mit Sulfadiazin dotiert und der oben beschriebenen Nachweismethode unterzogen (Tabelle 12 und Tabelle 13).

Tabelle 12: Wiederfindungsraten (\%) für Tetrazyklin (TC) und Chlortetrazyklin (CTC) in Sickerwasser bei Kalibration mit internem Standard

\begin{tabular}{lcccc}
\hline $\begin{array}{l}\text { Konzentration in der Probe } \\
\left(\boldsymbol{\mu g ~ \mathbf { ~ } ^ { - 1 } )}\right.\end{array}$ & $\begin{array}{c}\text { TC } \\
\mathbf{( \% )}\end{array}$ & STD & $\begin{array}{c}\text { CTC } \\
(\mathbf{\%})\end{array}$ & STD \\
\hline 1 & 116 & 14 & 115 & 16 \\
10 & 120 & 3 & 123 & 6 \\
40 & 129 & 17 & 128 & 18 \\
\hline Mittelwert $^{1}$ & 121 & 5 & 122 & 5 \\
\hline
\end{tabular}

${ }^{1}$ Mittelwert aus drei Messwiederholungen je Konzentration STD: Standardabweichung

Die mittlere Wiederfindungsrate betrug $121,3 \%$ für Tetrazyklin und $122,1 \%$ für Chlortetrazyklin.

Tabelle 13: Wiederfindungsrate (\%) von Sulfadiazin in Sickerwasser bei Kalibration mit internem Standard

\begin{tabular}{|c|c|c|c|c|c|}
\hline $\begin{array}{l}\text { Konzentration in der Probe } \\
\left(\mu \mathrm{g} \mathrm{l}^{-1}\right)\end{array}$ & $\begin{array}{l}\text { Probe } 1 \\
(\%)\end{array}$ & $\begin{array}{c}\text { Probe } 2 \\
(\%)\end{array}$ & $\begin{array}{c}\text { Probe } 3 \\
(\%)\end{array}$ & $\begin{array}{l}\text { MW } \\
(\%)\end{array}$ & STD \\
\hline 1 & 106 & 106 & 90 & 100 & 7 \\
\hline 2 & 71 & 109 & 95 & 91 & 16 \\
\hline
\end{tabular}

STD: Standardabweichung

MW: Mittelwert

So ergab sich für Sulfadiazin eine mittlere Wiederfindung von $95,9 \%$, die als Korrekturfaktor diente. Die in Kapitel 4.3 dargestellten Ergebnisse enthalten diese Korrekturfaktoren.

\subsection{Persistenz von Tetrazyklin in Masthähnchenfestmist}

Die Untersuchungen zur Persistenz von Tetrazyklin in Masthähnchenfestmist wurden mit handelsüblichen Kunststoffbehältern zur Schnellkompostierung durchgeführt (Abbildung 7). Diese Komposter gewährleisten eine Wärmedämmung durch die rundum doppelwandig 
ausgeführte Konstruktion. Die Behälter wurden anstelle einer Mietenlagerung gewählt, da so trotz der geringen Festmistmenge eine Kompostierung des Materials und eine Begrenzung der aufgrund der geringen Kompostmenge relativ gioßen Oberfläche möglich waren. Der als Kunststoffnetz (Maschenweite $10 \mathrm{~mm}$ ) ausgeführte Boden der Komposter gewährleistete eine passive Belüftung von unten. Die Lagerung fand in einem vor Wind, Regen und starken Temperaturschwankungen geschützten Raum statt

Die Persistenz von Tetrazyklin wurde in zwei Durchgängen untersucht, welche sich im experimentellen Aufbau unterschieden. Daher wird im Folgenden eine Unterteilung in Experiment 1 und 2 vorgenommen.
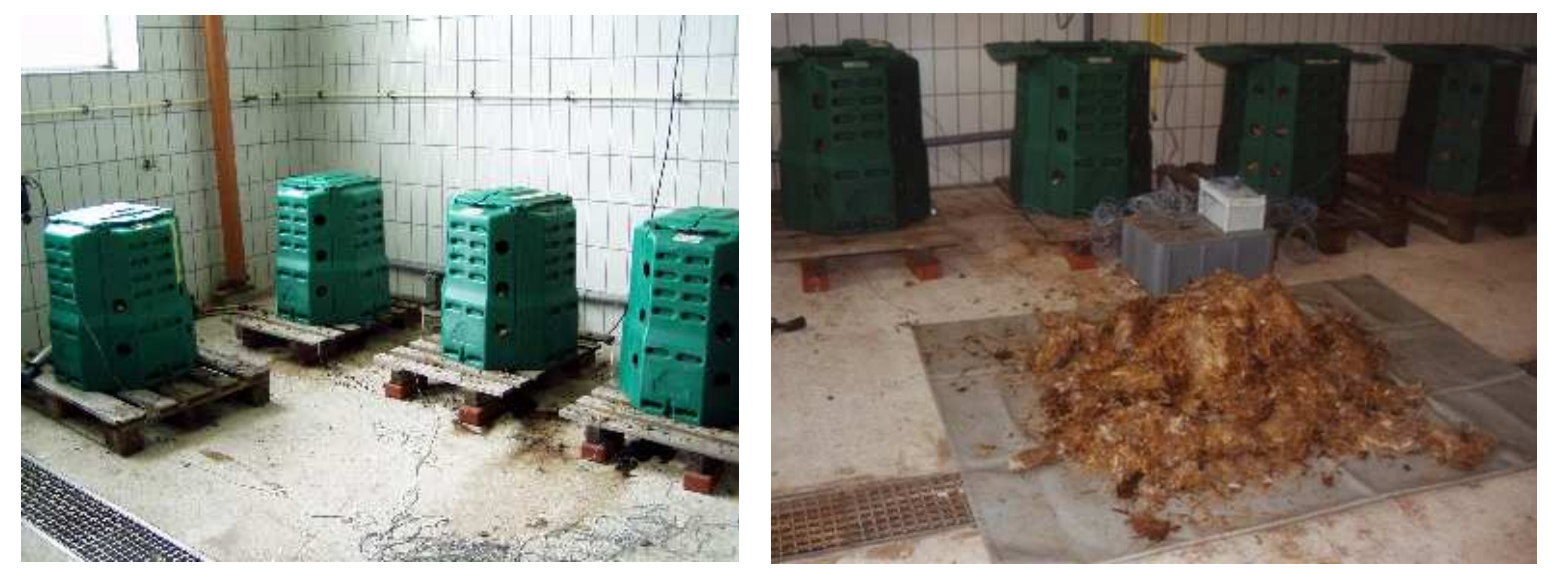

Abbildung 7: Kompostbehälter und durchmischter Masthähnchenfestmist beim Umsetzen der locker geschichteten Varianten

\subsubsection{Versuchsaufbau und Vorgehensweise von Experiment 1}

Der für den ersten Durchgang verwendete Festmist stamme aus einem zuvor durchgeführten Mastdurchgang (30 Masttage) mit Hähnchen der Herkunft Ross. Insgesamt 450 Masthähnchen wurden in sechs Gruppen á 75 Tiere aufgeteilt und in sechs Abteilen gehalten (Besatzdichte: 19 Tiere/ $\mathrm{m}^{2}$ ). Je zwei der sechs Gruppen erhielten über das Futter Tetrazyklin in einer Dosierung von 0,5 bzw. 1,0 g je kg Starterfutter über die ersten zehn Lebenstage, zwei Kontrollgruppen erhielten keine Antibiotika.

Der angefallene Festmist wurde nach der Ausstallung der Tiere über einen Zeitraum von drei Monaten als gestapelter Festmist bzw. als locker geschichteter Festmist gelagert. Die Lagerung erfolgte von Mitte März 2002 bis Anfang Juni 2002. Je Gruppe wurde der Festmist in einen Kompostbehälter (Fassungsvermögen 165 Liter) gefüllt. Je 
Fütterungsvariante wurde der Mist entweder als Stapelmist oder als locker geschichtete Variante (im Folgenden Rottemist genannt) gelagert, ebenso wurden auch die wirkstofffreien Kontrollen behandelt. Die Rottemist-Varianten wurden in der Mitte der Lagerungszeit, also nach sechs Wochen, einmal gründlich durchmischt und neu eingefüllt (Abbildung 7). So sollte eine gute Sauerstoffversorgung des Substrats erreicht werden. Die Stapelmist-Varianten dagegen wurden beim Befüllen der Behälter stark komprimiert und nicht umgesetzt.

Insgesamt erfolgte aufgrund der starken Verdunstung eine zusätzliche Wassergabe von zwölf Liter je Komposter über die Lagerungsdauer verteilt, um den Rotteprozess zu gewährleisten. Um den Abbau des Tetrazyklins bestimmen zu können, wurden im Abstand von zwei Wochen Proben aus den Kompostern entnommen. Da eine vollständige Homogenisierung des Masthähnchenfestmistes zu diesem Zweck durch die feste Lagerungsstruktur nicht möglich war, erfolgte die Probennahme mittels einer eigens angefertigten elektrisch betriebenen Fräse aus zwei unterschiedlichen Höhen der Komposter. Diese wurden als Mischproben weiter behandelt. Die Behälter waren zu diesem Zweck vor der Befüllung mit Bohrlöchern versehen worden. Direkt aus der Mitte des Lagergutes wurde eine ca. $3 \mathrm{~cm}$ dicke Mistprobe entnommen. An jedem Probennahmetermin wurde über einen anderen Zugang in den Mist gefräst, um stets ungestörte Proben zu erhalten. Das nicht benötigte Probenmaterial wurde in das jeweilige Fräsloch zurückgegeben. In Tabelle 14 sind die Versuchsanordnung und -bedingungen wiedergegeben.

Tabelle 14: Übersicht zu Versuchsanordnung und-bedingungen Experiment $1(\mathrm{~V}=$ Variante, $\mathrm{TC}=$ Tetrazyklin)

\begin{tabular}{lll}
\hline Parameter & Rottemist (RM) & Stapelmist (SM) \\
\hline Aufteilung des Festmistes & V 0,5 g TC/kg Futter & V 0,5 g TC/kg Futter \\
& V 1,0 g TC/kg Futter & V 1,0 g TC/kg Futter \\
& V Kontrolle & V Kontrolle \\
Temperatur & Raumlufttemperatur & Raumlufttemperatur \\
Lagerdauer & 12 Wochen & 12 Wochen \\
Umsetztermin & nach 6 Wochen & nicht umgesetzt \\
Probennahmeintervall & 14 Tage & 14 Tage \\
Probenart & Mischprobe aus 2 Höhen & Mischprobe aus 2 Höhen \\
\hline
\end{tabular}


Zur Erfassung der Umgebungs- und der Lagerungstemperatur in den Kompostern wurden insgesamt 13 Temperaturfühler an einen Datenlogger angeschlossen. Ein Fühler registrierte die Umgebungstemperatur und je zwei Fühler pro Komposter wurden in unterschiedlichen Höhen in die Kernzone des Festmistes eingebracht. Abbildung 8 zeigt die Anordnung der Versuchsglieder.

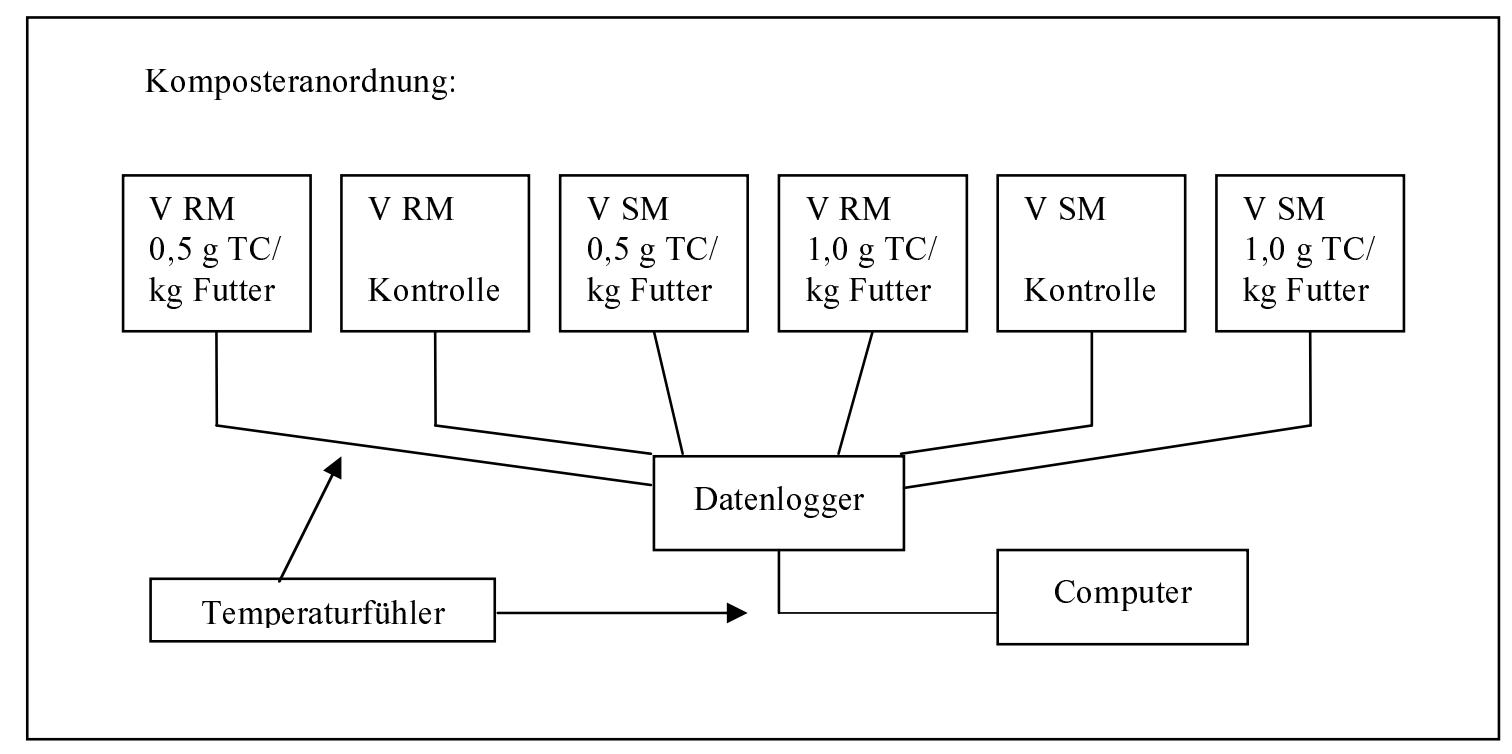

Abbildung 8: Schematische Darstellung der Versuchsanordnung beim Lagerungsversuch $(\mathrm{RM}=$ Rottemist, $\mathrm{SM}=$ Stapelmist, $\mathrm{V}=$ Variante, $\mathrm{TC}=$ Tetrazyklin $)$

\subsubsection{Versuchsaufbau und Vorgehensweise von Experiment 2}

Der für den zweiten Durchgang verwendete Masthähnchenfestmis stammte aus einem Mastbetrieb aus der Region Weser-Ems. Direkt nach dem Ausstallen der Masthähnchen wurden ca. $1300 \mathrm{~kg}$ Festmist entnommen. Zwischen dem Ausstallen und dem Befüllen der Komposter lag wie in Experiment 1 nur ein Tag. Der gesamte Festmist wurde durch einen Miststreuer zerkleinert sowie gemischt und auf diese Weise homogenisiert (Abbildung 9). Insgesamt standen im zweiten Experiment acht Komposter zur Verfügung. Zwei Komposter wurden mit dem Ausgangsmist befüllt und dienten als Kontrollen. Der restliche Mist wurde zu je $100 \mathrm{~kg}$ portioniert und jeweils mit in 51 Wasser gelöstem Tetrazyklin auf $45 \mathrm{mg} \mathrm{kg}^{-1}$ eingestellt. Nach einer gründlichen Homogenisierung mittels Rührgerät (Firma Beba Mischtechnik, Handmischer B7) wurden jeweils drei Komposter als RottemistVarianten und drei Komposter als Stapelmist-Varianten mit dem Festmist befüllt (siehe Kapitel 3.2.1). Die Rottemist-Varianten wurden nach der Hälfte der Lagerungszeit einmal umgesetzt und dabei gründlich durchmischt. Die Lagerungsdauer betrug zwei Monate, von Mitte Mai 2003 bis Mitte Juli 2003. 

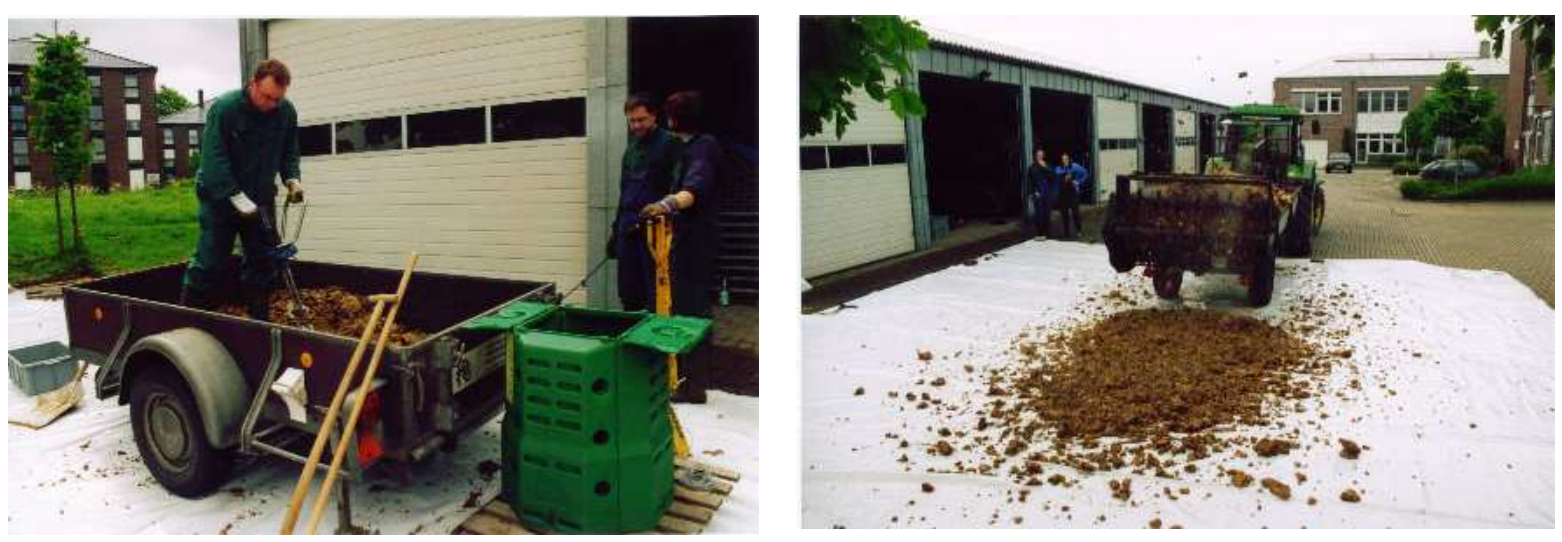

Abbildung 9: Homogenisierung, Befüllen der Komposter und Dotierung des Masthähnchenfestmistes mit Tetrazyklin

Die Probennahme und die Temperaturerfassung erfolgten wie in Experiment 1 beschrieben. Etwa 28 Liter Wasser wurden im Verlauf der Lagerung je Komposter zugesetzt, um den Kompostiervorgang nicht durch Substrattrockenheit zu gefährden. Tabelle 15 stellt die Versuchsanordnung und -bedingungen in einer Zusammenfassung dar. Die Anordnung der Versuchsglieder war vergleichbar mit Experiment 1 (siehe Kapitel 3.2.1).

Tabelle 15: Übersicht zu Versuchsanordnung und -bedingungen Experiment $2(\mathrm{~V}=$ Variante)

\begin{tabular}{lll}
\hline Parameter & Rottemist (RM) & Stapelmist (SM) \\
\hline Aufteilung des Festmistes & RM KT: Kontrolle & SM KT: Kontrolle \\
& RM 1-3 : ca. 45 mg TC kg ${ }^{-1}$ & SM 1-3: ca. 45 mg TC kg ${ }^{-1}$ \\
Temperatur & Raumlufttemperatur & Raumlufttemperatur \\
Lagerdauer & 8 Wochen & 8 Wochen \\
Umsetztermin & nach 4 Wochen & nicht umgesetzt \\
Probennahmeintervall & 14 Tage & 14 Tage \\
Probenart & Mischprobe aus 2 Höhen & Mischprobe aus 2 Höhen \\
\hline
\end{tabular}

\subsection{Screening von Schweinegülleproben auf ausgewählte Antibiotika}

Für die Untersuchung von Schweinegülle aus landwirtschaftlichen Praxisbetrieben standen zwei Probensätze zur Verfügung: 168 Einzelproben stammten aus dem Winterhalbjahr 1999/2000; ein weiterer Probensatz mit 176 Einzelproben konnte im Winterhalbjahr 2001/2002 gewonnen werden. Herkunftsgebiet war die Weser-Ems-Region. 
Die Proben wurden anonym zur Verfügung gestellt, so dass keine Angaben über den Betrieb, die Art und den Ort der Probennahme (z.B. Aufrühren der Gülle, Probe aus Stall oder Güllebehälter) oder über einen möglichen Medikamenteneinsatz vorlagen. Es ist weiterhin davon auszugehen, dass in der überwiegenden Zahl der Fälle die Probennahme durch den Landwirt selbst erfolgte. Bis auf wenige Ausnahmen standen die Trockensubstanz- und Stickstoffgehalte zur Verfügung. Die Proben wurden bis zur Analyse auf Tetrazyklin, Oxy- und Chlortetrazyklin, Sulfadiazin und Sulfamethazin bei $-18{ }^{\circ} \mathrm{C}$ gelagert.

Proben aus dem Probensatz des Jahres 2001/2002, die nach Analyse durch unter Kapitel 3.1.2 beschriebene Methode antibiotikafrei waren, wurden in der Zentrumsabteilung für Lebensmitteltechnologie und -toxikologie der Tierärztlichen Hochschule Hannover einem noch sensitiveren Nachweisverfahren nach Hamscher et al. (2000) unterzogen. In dieser Methode wird mit einer Flüssig-Flüssigextraktion gearbeitet, wonach eine flüssigkeitschromatographische Trennung (HPLC) erfolgt. Beim Verlassen der Trennsäule werden die Substanzen mittels Elektrospray-Tandemmassenspektrometrie (LC-ESI-MSMS) bestimmt. Die Bestimmungsgrenzen liegen mit dieser Methode für Tetrazykline und Sulfonamide bei $0,05 \mathrm{mg} \mathrm{kg}^{-1}$ Frischgülle. In die spätere Auswertung gingen lediglich die Proben des Jahres 2001/2002 ein, die Antibiotikakonzentrationen bis zur Bestimmungsgrenze wie unter Kapitel 3.1.2 beschrieben aufwiesen.

\subsection{Mobilität von Tetrazyklin und Sulfadiazin in Böden}

Die Mobilität von Tetrazyklin und Sulfadiazin in verschiedenen Böden wurde in Zusammenarbeit mit der Landwirtschaftskammer Weser-Ems in Oldenburg unter Nutzung der dort vorhandenen Kick-Brauckmann-Gefäße und Lysimeter untersucht.

\subsubsection{Versuchsaufbau und Vorgehensweise Kick-Brauckmann-Gefäße}

Je 16 Kick-Brauckmann-Gefäße (Ø $22 \mathrm{~cm}$, Krumenhöhe $24 \mathrm{~cm})$ wurden mit zwei für die Weser-Ems-Region typischen Böden (humoser Sand, 7,5 kg/Gefäß; lehmiger Schluff, $8 \mathrm{~kg} / \mathrm{Gefäß)}$ sowie einem industriell hergestellten Torfgemisch (sogenannte Null oder Einheitserde, $3 \mathrm{~kg} / \mathrm{Gefäß)}$ befüllt. Nullerde besteht zu $70 \%$ aus Weißtorf, zeichnet sich also durch einen hohen Strukturanteil aus (weitere Bestandteile sind Ton und Kalk) und wurde mangels einer geeigneten Moorbodenfläche herangezogen. Die Böden waren wie folgt gekennzeichnet (Tabelle 16): 
Tabelle 16: Kenndaten der verwendeten Böden im Gefäßversuch

\begin{tabular}{lccc}
\hline Kenndaten & Humoser Sand & Lehmiger Schluff & Nullerde \\
\hline Bodennutzung & Acker & Acker & Gärtnertorf \\
pH-Wert & 5,0 & 5,5 & 5,9 \\
Kalzium (mg/100 g) & 100 & 100 & 1050 \\
Humusanteil (\%) & 6,0 & 3,1 & 30,3 \\
Tonanteil (\%) & 1,3 & 6,6 & k.A. \\
Schluffanteil (\%) & 13,2 & 63,2 & k.A. \\
KAK ${ }^{1}$ (mval/100 g) & 21,3 & 12,6 & 84,8 \\
\hline${ }^{1} \mathrm{KAK}^{-}$Kationaustauschapaziöt & &
\end{tabular}

Die Gefäße wurden ohne Bewuchs bei etwa 50\% Feldkapazität gehalten (Abbildung 10). Diese Bedingung konnte durch die Ausnutzung der Saugspannung mittels Verdunstung gewährleistet werden. Dazu wurden Glasfaserstränge in das Bodenmaterial eingearbeitet, die in Wasserbehältern endeten. Aufgrund der Saugspannung wurde Wasser über die Glasfaserstränge in den Boden gesogen, der auf diese Weise feucht blieb. Es handelte sich bei der Glasfaser um ein inertes Material, so dass keine Wechselwirkungen mit den beiden Antibiotika zu erwarten waren. Labortests mit Tetrazyklin und Sulfadiazin bestätigten, dass auch bei längerer Einwirkzeit keine Anlagerung der Wirkstoffe an die Glasfasern auftrat.

Für den Versuch wurde nachweislich wirkstofffreie Gülle mit Tetrazyklin und Sulfadiazin in einer Konzentration von jeweils $40 \mathrm{mg} \mathrm{kg}^{-1}$ versetzt. Insgesamt 18 der Gefäße (sechs Gefäße je Boden) wurden mit jeweils $200 \mathrm{ml}$ der tetrazyklinhaltigen Gülle (40 mg TC kg-1) dotiert, was einer Menge von $8 \mathrm{mg}$ TC je Gefäß entsprach. Weitere 18 Gefäße erhielten jeweils $200 \mathrm{ml}$ sulfadiazinhaltige Gülle (40 mg SD kg${ }^{-1}$ ), was ebenfalls eine applizierte Sulfadiazinmenge von $8 \mathrm{mg}$ je Gefäß ergab. Die Güllemenge von $200 \mathrm{ml}$ je Gefäß entsprach einer Menge von $43 \mathrm{~m}^{3}$ je Hektar bzw. $179 \mathrm{~kg} \mathrm{~N}^{-1}$. Die restlichen Gefäße dienten als Kontrollen.

Bei der Aufbringung der Gülle wurde ein Einarbeiten des Wirtschaftsdüngers nachgeahmt, indem mit einem Pikierstab je Gefäß drei ca. 1,5 cm breite Furchen gezogen wurden. Die Gülle wurde in die Furchen gegeben und diese mit dem aufgeworfenen Bodenmaterial bedeckt. Auf diese Weise wurde auch sichergestellt, dass keine Gülle an den Gefäßrand gelangen und dort möglicherweise am Kunststoff adsorbieren konnte. 
Das Verhalten der Wirkstoffe wurde zu zwei unterschiedlichen Zeitpunkten untersucht um möglichen Abbau- oder Bindungsvorgängen im Boden Rechnung zu tragen.
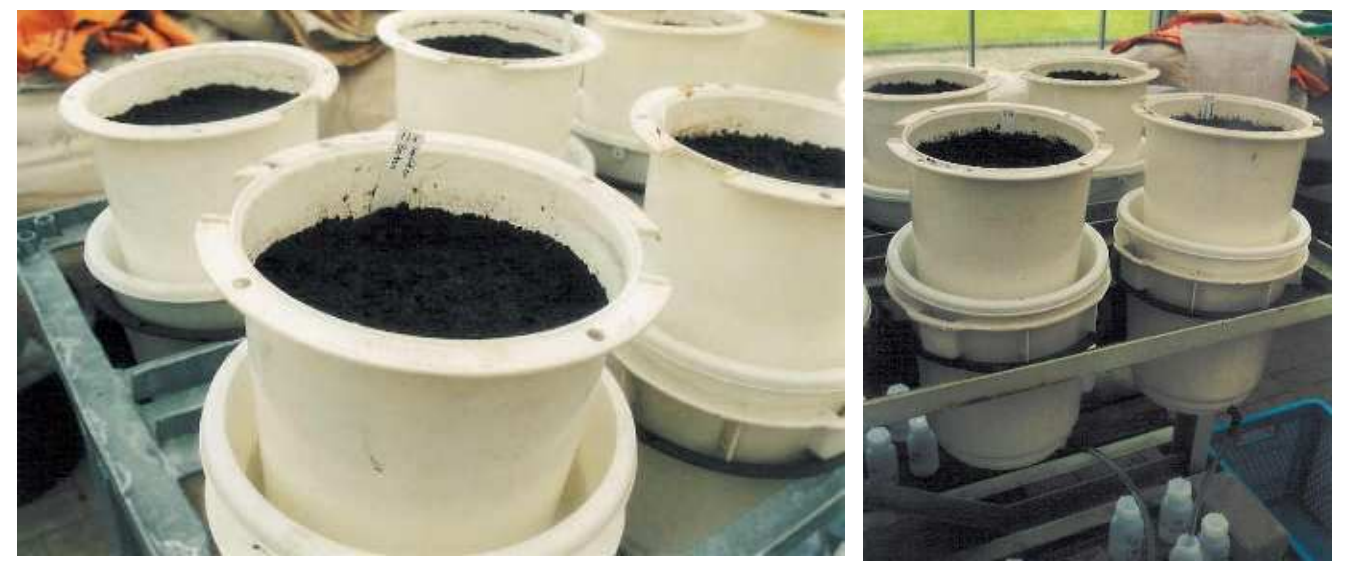

Abbildung 10: Kick-Brauckmann-Gefäße und Beprobungsvorrichtung zum Auffangen der Sickerwasserfraktionen

\section{Starkregensimulation:}

48 Stunden nach Versuchsansatz wurden die Hälfte der mit Tetrazyklin und Sulfadiazin dotierten Gefäße (drei je Bodenart und Behandlung) sowie sechs Kontrollgefäße mit 1,52 Litern Wasser je Gefäß über eine Zeitspanne von 1,5 Stunden beregnet. Damit wurde ein Starkregenereignis von $401 / \mathrm{m}^{2}$ simuliert.

Zehn Wochen nach Versuchsansatz wurden die restlichen 24 Gefäße in der gleichen Art beregnet.

\section{Probennahme:}

Das nach der Beregnung erhaltene Sickerwasser je Gefäß wurde in vier Fraktionen zu je $100 \mathrm{ml}$ aufgefangen (Probe I-IV) und bis zur Analyse bei $-18^{\circ} \mathrm{C}$ gelagert. Darüber hinaus angefallenes Sickerwasser wurde nicht in die Analyse miteinbezogen. Zusätzlich erfolgte jeweils nach der Beregnung die Entnahme von Proben des Ober und Unterbodens (Oberboden 0-12 cm, Unterboden 12-24 cm; je Gefäß drei Einstiche, daraus Mischprobe). Bei den mit Sulfadiazin dotierten Gefäßen wurden nur die Proben aus dem Oberboden analysiert, da die Verlagerbarkeit des Sulfadiazins in den Unterboden als sehr gering eingestuft wurde.

Die Analyse der Bodenproben sowie die Referenzmessung von Sickerwasserproben erfolgten nach Hamscher et al. (2002) in der Zentrumsabteilung für Lebensmitteltechnologie und -toxikologie der Tierärztlichen Hochschule Hannover. Die 
Bestimmungsgrenze dieses Verfahrens lag bei $0,1 \mu \mathrm{g} \mathrm{l}^{-1}$ für Tetrazyklin und Sulfadiazin in Sickerwasser und bei $5 \mu \mathrm{g} \mathrm{kg}^{-1}$ in Boden.

\subsubsection{Versuchsaufbau und Vorgehensweise Lysimeter}

Die hier bearbeitete Fragestellung zum Verhalten von Tetrazyklin in Böden konnte in eine seit mehreren Jahren durchgeführte Versuchsreihe zur Frage der Stickstoffauswaschung bei unterschiedlichen Gülle-N-Gaben (0, 80, 120, 160 bzw. $240 \mathrm{~kg} \mathrm{~N})$ integriert werden. Dafür standen im Zeitraum zwischen Frühjahr 2001 und Oktober 2002 insgesamt 20 Lysimeter zur Verfügung. Die Lysimeter (Tiefe: 1,50 m; Ø $1 \mathrm{~m}$ ) wurden bereits $1988 \mathrm{mit}$ einem für die Region typischen humosen Sand befüllt. Zehn der Lysimeter waren mit der Fruchtart Winterroggen bestellt, die anderen zehn mit der Fruchtart Mais (Abbildung 11). Zusätzlich konnten in 2001 vier weitere Lysimeter, seit 1996 mit einem humosen Sand anderer Herkunft befüllt, genutzt werden (Tabelle 17). Diese Lysimeter wiesen als Bewuchs Mais auf.

Tabelle 17: Kenndaten der in den Lysimetern verwendeten Böden, aufgeteilt nach Oberboden (Krume) und Unterboden

\begin{tabular}{lcc}
\hline Kenndaten & Humoser Sandboden I & Humoser Sandboden II \\
\hline Startzeit & 1988 & 1996 \\
Bodennutzung & Acker & Acker \\
$\mathrm{pH}-W e r t$ & $5,2^{1}-5,0^{2}$ & $5,1^{1}-5,0^{2}$ \\
$\mathrm{Humusanteil}(\%)$ & $2,4^{1}-0,5^{2}$ & $3,6^{1}-4,6^{2}$ \\
$\mathrm{~K} 2 \mathrm{O}(\mathrm{mg} / 100 \mathrm{~g})$ & $15^{1}-6^{2}$ & $32^{1}-24^{2}$ \\
$\mathrm{Mg}(\mathrm{mg} / 100 \mathrm{~g})$ & $3^{1}-1^{2}$ & $11^{1}-7^{2}$ \\
\hline${ }^{1} \mathrm{Gehalt}$ im Oberboden (Krume) & & \\
${ }^{2} \mathrm{Gehalt}$ im Unterboden & & \\
$\mathrm{Mg} \quad$ Magnesium & & \\
$\mathrm{K}_{2} \mathrm{O} \quad=$ Kaliumoxid & &
\end{tabular}

Nachweislich wirkstofffreie Schweinegülle (N-Gehalt $=0,42 \%)$ wurde im ersten Versuchsjahr (2001) mit $15 \mathrm{mg} \mathrm{kg}^{-1}$ Tetrazyklin dotiert. Analog zu den N-Düngungsstufen 80,160 und $240 \mathrm{~kg} \mathrm{~N} \mathrm{ha}^{-1}$ wurden über die erforderlichen unterschiedlichen Güllegaben unterschiedliche Tetrazyklinmengen von 22,5 bis $67,2 \mathrm{mg}$ je Lysimeter aufgebracht (Tabelle 18). Bei Mais wurde die Gülle vor der Aussaat ca. 3 - $4 \mathrm{~cm}$ tief in den Oberboden eingearbeitet, bei Winterroggen erfolgte im Frühjahr eine oberftächliche Ausbringung in den Bestand. Vier zusätzliche Lysimeter der N-Stufe $200 \mathrm{~kg} \mathrm{~N} \mathrm{ha}^{-1}$ mit humosem 
Sandboden II erhielten mit 18 bzw. $36 \mathrm{mg} \mathrm{kg}^{-1}$ dotierte Schweinegülle (entsprechend 67,3 bzw. 134,6 mg Tetrazyklin).
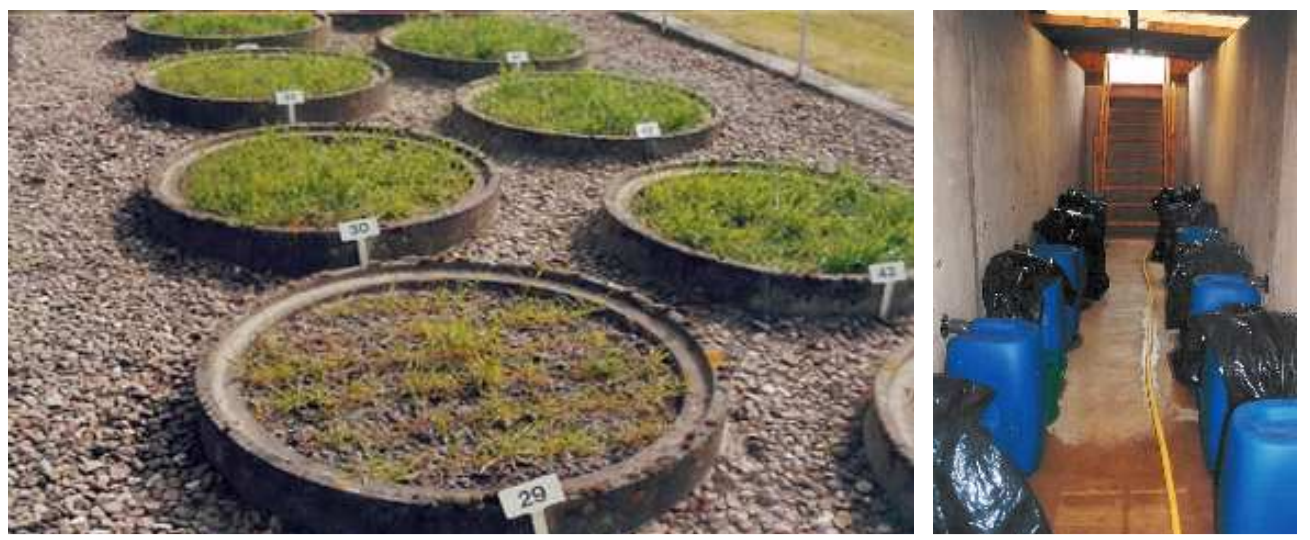

Abbildung 11: Mit Winterroggen bestellte Lysimeter und Lysimetergang mit Sickerwasserauffangbehältern

Tabelle 18: Übersicht zur Versuchsanordnung bei der Untersuchung der Mobilität von Tetrazyklin und Sulfadiazin im Lysimeter $(\mathrm{TC}=$ Tetrazyklin; $\mathrm{SD}=$ Sulfadiazin; N = Stickstoff))

\begin{tabular}{|c|c|c|c|c|c|c|c|}
\hline \multirow[t]{2}{*}{ Kultur } & \multirow[t]{2}{*}{$\begin{array}{l}\text { Lys.- } \\
\text { Nr. }\end{array}$} & \multirow[t]{2}{*}{$\begin{array}{l}\text { N-Stufe } \\
\left(\mathrm{kg} \mathrm{ha}^{-1}\right)\end{array}$} & \multicolumn{2}{|c|}{$\begin{array}{c}\text { Güllemenge } \\
\text { (I) }\end{array}$} & \multicolumn{2}{|c|}{$\begin{array}{l}\text { TC-Menge } \\
\text { (mg/Lys.) }\end{array}$} & \multirow{2}{*}{$\begin{array}{r}\text { SD-Menge } \\
\text { (mg/Lys.) }\end{array}$} \\
\hline & & & 2001 & 2002 & 2001 & 2002 & \\
\hline \multirow{7}{*}{$\begin{array}{c}\text { M } \\
\text { A } \\
\text { I } \\
\text { S }\end{array}$} & $1+25$ & 0 & 0 & 0 & 0 & 0 & 0 \\
\hline & $2+26$ & 80 & 1,50 & 1,85 & 22,5 & 22,2 & 22,2 \\
\hline & $3+27$ & 120 & 2,24 & 2,77 & 0 & 0 & 0 \\
\hline & $4+28$ & 160 & 3,00 & 3,69 & 45,0 & 44,3 & 44,3 \\
\hline & $13+35$ & 240 & 4,48 & 5,54 & 67,2 & 66,5 & 66,5 \\
\hline & $49+61 *$ & 200 & 3,74 & - & 67,3 & - & - \\
\hline & \multirow[t]{2}{*}{$58+65^{*}$} & 200 & 3,74 & - & 134,6 & - & - \\
\hline & & & 2001 & 2002 & 2001 & 2002 & 2002 \\
\hline $\mathbf{R}$ & $5+29$ & 0 & 0 & 0 & 0 & 0 & 0 \\
\hline $\begin{array}{l}\mathbf{O} \\
\mathbf{n}^{2}\end{array}$ & $6+30$ & 80 & 1,50 & 1,85 & 22,5 & 22,2 & 22,2 \\
\hline G & $7+31$ & 120 & 2,24 & 2,77 & 0 & 0 & 0 \\
\hline $\mathbf{E}$ & $8+32$ & 160 & 3,0 & 3,69 & 45,0 & 44,3 & 44,3 \\
\hline & $15+33$ & 240 & 4,48 & 5,54 & 67,2 & 66,5 & 66,5 \\
\hline
\end{tabular}

* Bodenart: humoser Sand II (vier zusätzliche Lysimeter)

${ }^{1}$ in 2001 kein Sulfadiazin eingesetzt 
Je Wirkstoffmenge und Fruchtart wurden zwei Lysimeter eingesetzt. Weiterhin standen als Kontrolle jeweils zwei mit wirkstofffreier Gülle gedüngte Lysimeter (120 kg N-Stufe) sowie zwei Lysimeter, die keine Gülle erhielten, zur Verfügung.

Im zweiten Versuchsjahr (2002) erfolgte mit Ausnahme der vier zusätzlichen Lysimeter mit humosem Sandboden II eine Wiederholung. Zusätzlich wurde Sulfadiazin als weiteres Tierarzneimittel zugesetzt. Aufgrund eines geringeren N-Gehalts der auszubringenden Gülle wurde 2002 jeweils eine Konzentration von $12 \mathrm{mg} \mathrm{kg}^{-1} \mathrm{TC}$ bzw. SD eingestellt.

\section{Probennahme:}

Das Sickerwasser aus den Lysimetern wurde in lichtgeschützten Behältern aufgefangen, da Tetrazyklin bei Lichteinwirkung instabil ist (Kühne et al. 2001). Wöchentlich wurde die angefallene Gesamt-Sickerwassermenge erfasst und eine Probe des Sickerwassers (ca. $500 \mathrm{ml}$ ) entnommen. Die Wasserproben wurden umgehend eingefroren und bis zur weiteren Verwendung bei $-18^{\circ} \mathrm{C}$ gelagert.

Im ersten Versuchsjahr (2001) wurde bei Roggen ab der 13. Kalenderwoche (KW) mit der Beprobung begonnen, bei Mais ab der 19. KW (erste Sickerwasserbildung nach Gülleaufbringung). In der 50. KW 2001 endete die Sickerwasserbildung aufgrund des starken Frostes. Im 2. Versuchsjahr 2002 wurde bei Roggen ab der 16. KW beprobt, bei Mais erfolgte die Probennahme ab der 21. KW; die letzte Probennahme fand in der 40. KW statt.

Zusätzlich zu den Wasserproben wurden im Frühjahr und im Herbst von ausgewählten Lysimetern Bodenproben entnommen und von der Zentrumsabteilung für Lebensmitteltechnologie und -toxikologie der Tierärztlichen Hochschule Hannover nach Hamscher et al. (2002) auf Tetrazyklin bzw. Sulfadiazin untersucht (siehe Kapitel 3.4.1). Die Probennahmetiefe betrug $20 \mathrm{~cm}$. Es wurden acht Einstiche je Lysimeter vorgenommen und der so erhaltene Boden als Mischprobe weiter behandelt.

\subsection{Weitere Analysemethoden und statistische Auswertung}

\section{Masthähnchenfestmist}

Im frischen Festmist wurde in Anlehnung an die Methoden der Bundesgütegemeinschaft Kompost e.V. der pH-Wert gemessen: $20 \mathrm{~g}$ Probematerial wurde mit $200 \mathrm{ml}$ 0,01 M $\mathrm{CaCl}_{2}$-Lösung versetzt und der $\mathrm{pH}$-Wert nach einer Stunde gemessen (IKA Labortechnik; Mettler Toledo Inlab 417). 
Beim getrockneten und gemahlenen Festmist wurden die Gehalte von Trockenmasse, Asche, Gesamtkohlenstoff und Gesamtstickstoff bestimmt. Die Aschegehalte wurden bei $550{ }^{\circ} \mathrm{C}$ im Muffelofen ermittelt; die Trockenmassegehalte durch den Masseverlust bei der Gefriertrocknung.

Mit dem LECO SC-444 (Firma LECO, Kirchheim) erfolgte die Gesamtkohlenstoffmessung. $100 \mathrm{mg}$ des getrockneten Probenmaterials wurde bei $950{ }^{\circ} \mathrm{C}$ im Sauerstoffstrom in zwei Heiz- und Verbrennungszonen verbrannt. In der ersten Zone erfolgten die thermische Aktivierung der Probe und die Aufoxidierung zu CO2. Die zweite Zone diente der Oxidierung der nicht vollständig umgesetzten Kohlenstoffkomponenten wie $\mathrm{Ru}$, Kohlenwasserstoffe oder $\mathrm{CO}$ zu $\mathrm{CO}_{2}$.

Der Gesamtstickstoff wurde mit dem Macro-N der Firma Elementar-Analysensysteme, Hanau, bestimmt. Der Probenaufschluss erfolgte nach der modifizierten Methode von DUMAS: etwa 0,75 bis 1,0 g der Probe wurden eingewogen und im Analysegerät durch oxidative Verbrennung in mit $\mathrm{O}_{2}$ angereicherter $\mathrm{CO}_{2}$-Atmosphäre aufgeschlossen. Die entstandenen gasförmigen Zersetzungsprodukte wurden anschließend im Nachverbrennungsrohr an einem $\mathrm{CuO} / \mathrm{Pt}-$ Katalysator quantitativ umgesetzt. Das Gasgemisch aus $\mathrm{CO}_{2}, \mathrm{H}_{2} \mathrm{O}$ und elementarem Stickstoff unterlag einer Trocknung und gelangte dann zur Detektionseinheit (Wärmeleitfähigkeitsdetektor).

\section{Sickerwasser}

Ausgewählte Sickerwasserproben wurden mit dem Atomabsorptionsspektrometer (AAS) (Varian, SpectrAA-300) auf ihre Kalziumgehalte untersucht. In Lösung befindliches Kalzium wurde mittels Flammen-AAS gemessen. Hierbei wird die Lösung in eine Flamme gestäubt, in der die Kalziumionen zu einem gewissen Anteil atomisiertwerden und somit Licht einer für Kalzium spezifischen Wellenlänge absorbieren. Bei steigender Kalziumkonzentration ist die Absorption umso größer; auf Basis dieser Absorption erfolgt die Quantifizierung.

\section{Statistik}

Die statistische Datenauswertung erfolgte mit Hilfe des Softwarepakets SPSS (Version 11.5). Hauptsächlich wurden beschreibende Methoden der Statistik verwendet wie die Berechnung der Mittelwerte mit der jeweiligen Standardabweichung, des Medians und der Minimal- und Maximalwerte. In Kapitel 3.1.2 und 4.1 wurde die Varianzanalyse als Methode der schließenden Statistik sowie die ehrlich signifikante Differenz nach Tukey als 
Test für Mehrfachvergleiche eingesetzt. Mit dem nicht-parametrischen Kruskall-WallisTest und dem Wilcoxon-Mann-Whitney-U-Test wurden in Kapitel 4.2 Gruppen verschiedener Trockensubstanzklassen der Schweinegülleproben auf Unterschiede untersucht. Eine Regressionsanalyse wurde in Kapitel 5.2 durchgeführt, um Halbwertszeiten für Tetrazyklin in gelagertem Masthähnchenfestmist zu ermitteln. 


\section{Ergebnisse}

\subsection{Modellversuche zur Persistenz von Tetrazyklin in Masthähnchenfestmist}

\subsubsection{Experiment 1}

Der Masthähnchenfestmistversuch Experiment 1 enthielt vier unterschiedlichen TCAusgangskonzentrationen und zwei verschiedene Lagerungsarten: Rotte (RM)- und Stapelmist (SM). Tabelle 19 zeigt die Ausgangskenngrößen des Festmistes zu Beginn der Lagerung, in Tabelle 20 sind zum Vergleich die Kenngrößen nach Beendigung der Lagerung aufgeführt.

Tabelle 19: Stoffkenngrößen wie Stickstoff- (N), Kohlenstoff- (C), organischer Substanzgehalt (OS) des im Experiment 1 eingesetzten Masthähnchenfestmistes zu Beginn der Lagerung (in \% bezogen auf den TS-Gehalt)

\begin{tabular}{|c|c|c|c|c|c|c|}
\hline \multirow{2}{*}{$\begin{array}{l}\text { Stoffkenn- } \\
\text { größen }\end{array}$} & \multicolumn{6}{|c|}{ Lagerungsvarianten } \\
\hline & $\begin{array}{r}\mathbf{K T} \\
\mathbf{R M}\end{array}$ & $\begin{array}{l}\text { KT } \\
\text { SM }\end{array}$ & $\begin{array}{r}0,5 \mathrm{~g} \mathrm{TC} \\
\mathrm{kg}^{-1} \text { Futter } \\
\mathrm{RM}\end{array}$ & $\begin{array}{r}0,5 \text { g TC } \\
\mathbf{k g}^{-1} \text { Futter } \\
\mathrm{SM}\end{array}$ & $\begin{array}{r}1,0 \mathrm{~g} \mathrm{TC} \\
\mathrm{kg}^{-1} \text { Futter } \\
\text { RM }\end{array}$ & $\begin{array}{r}1,0 \mathrm{~g} \mathrm{TC} \\
\mathrm{kg}^{-1} \text { Futter } \\
\mathrm{SM}\end{array}$ \\
\hline TS (\%) & 50,4 & 50,5 & 48,6 & 48,6 & 52,9 & 53,9 \\
\hline $\mathrm{N}(\%)$ & 5,6 & 5,5 & 5,7 & 5,8 & 5,4 & 5,5 \\
\hline $\mathrm{C}(\%)$ & 41,3 & 41,1 & 41,5 & 40,7 & 41,0 & 40,7 \\
\hline OS $(\%)$ & 86,7 & 86,9 & 86,6 & 86,1 & 87,1 & 86,6 \\
\hline $\mathrm{C} / \mathrm{N}$ & $7,4: 1$ & $7,4: 1$ & $7,3: 1$ & $7,0: 1$ & $7,6: 1$ & $7,5: 1$ \\
\hline $\mathrm{TC}\left(\mathrm{mg} \mathrm{kg}^{-1}\right)$ & 0,0 & 0,0 & 26,0 & 44,1 & 55,7 & 100,1 \\
\hline Mist $(\mathrm{kg})^{1}$ & 76,6 & 88,6 & 78,3 & 88,7 & 72,4 & 93,2 \\
\hline $\begin{array}{l}=\text { Frisc } \\
=\text { Kont } \\
=\text { Stap } \\
=\text { Rotte } \\
=\text { Tetra }\end{array}$ & & & & & & \\
\hline
\end{tabular}

Durch das Verdichten wurde im Vergleich zu den Rottemistvarianten zwischen 10 und $15 \mathrm{~kg}$ mehr Mist in die Stapelmist-Varianten eingefüllt. Im Verlauf der Lagerung erhöhte sich der Trockensubstanzgehalt (TS-Gehalt) des Mistes um ca. $13 \%$. Um die für die stofflichen Umsetzungsprozesse notwendige Materialfeuchte zu gewährleisten, wurde das Lagergut von Zeit zu Zeit kontrolliert bewässert. Zur Berücksichtigung dieser externen Wasserzufuhr werden die Ergebnisse im Folgenden auf den Trockensubstanzgehalt bezogen. 
Tabelle 20: Stoffkenngrößen wie Stickstoff- (N), Kohlenstoff- (C), organischer Substanzgehalt (OS) des im Experiment 1 eingesetzten Masthähnchenfestmistes am Ende der Lagerung (in \% bezogen auf den TS-Gehalt)

\begin{tabular}{|c|c|c|c|c|c|c|}
\hline \multirow{2}{*}{$\begin{array}{l}\text { Stoffkenn- } \\
\text { größen }\end{array}$} & \multicolumn{6}{|c|}{ Lagerungsvarianten } \\
\hline & $\begin{array}{r}\text { KT } \\
\text { RM }\end{array}$ & $\begin{array}{l}\text { KT } \\
\text { SM }\end{array}$ & $\begin{array}{r}0,5 \mathrm{~g} \mathrm{TC} \\
\mathrm{kg}^{-1} \text { Futter } \\
\text { RM }\end{array}$ & $\begin{array}{r}0,5 \text { g TC } \\
k^{-1} \text { Futter } \\
\mathrm{SM}\end{array}$ & $\begin{array}{r}1,0 \mathrm{~g} \mathrm{TC} \\
\mathrm{kg}^{-1} \text { Futter } \\
\text { RM }\end{array}$ & $\begin{array}{r}1,0 \mathrm{~g} \mathrm{TC} \\
\mathrm{kg}^{-1} \text { Futter } \\
\mathrm{SM}\end{array}$ \\
\hline TS $(\%)$ & 68,1 & 51,1 & 62,3 & 60,7 & 65,7 & 65,3 \\
\hline $\mathrm{N}(\%)$ & 4,6 & 4,8 & 4,5 & 5,7 & 4,7 & 3,6 \\
\hline $\mathrm{C}(\%)$ & 36,5 & 38,0 & 35,9 & 37,0 & 36,5 & 34,6 \\
\hline OS $(\%)$ & 76,4 & 78,6 & 74,9 & 79,9 & 76,5 & 76,6 \\
\hline $\mathrm{C} / \mathrm{N}$ & $7,9: 1$ & $8,0: 1$ & $8,0: 1$ & $6,6: 1$ & $7,8: 1$ & $9,5: 1$ \\
\hline $\mathrm{TC}\left(\mathrm{mg} \mathrm{kg}^{-1}\right)$ & 0,0 & 0,0 & 3,5 & 0,0 & 9,8 & 22,0 \\
\hline $\begin{array}{l}=\text { Kont } \\
=\text { Stape } \\
=\text { Rotte } \\
=\text { Tetra }\end{array}$ & & & & & & \\
\hline
\end{tabular}

Die Fermentationsprozesse des Rotte- und Stapelmistes verliefen bei Kohlenstoff (C), Stickstoff $(\mathrm{N})$ und $\mathrm{C}: \mathrm{N}-$ Verhältnis in der Tendenz parallel, nur die organische Substanz (OS) wurde im Rottemist ca. 2 \% stärker abgebaut als im Stapelmist. Der pH-Wert lag zu Beginn der Lagerung im Festmist bei ca. 5,4. Im Verlauf der Lagerung stieg er bis auf etwa 8,7 an.

\section{Temperaturverlauf}

Die Lagerungsart hatte keinen signifikanten Einfluss auf die Temperaturentwicklung (Abbildung 12). Während des ersten Monats lag die Temperatur im Rottemist geringfügig über der des Stapelmistes, ab dem Umsetztermin trat dieser Effekt nur noch zeitweise auf (siehe Anhang, Tabelle 34). Die Schwankungen in den jeweiligen Temperaturverläufen an Tag 36, 43, 46, 58 und 72 sind auf die kontrollierte Zugabe von Wasser und die dadurch angeregten Zersetzungsprozesse zurückzuführen. 


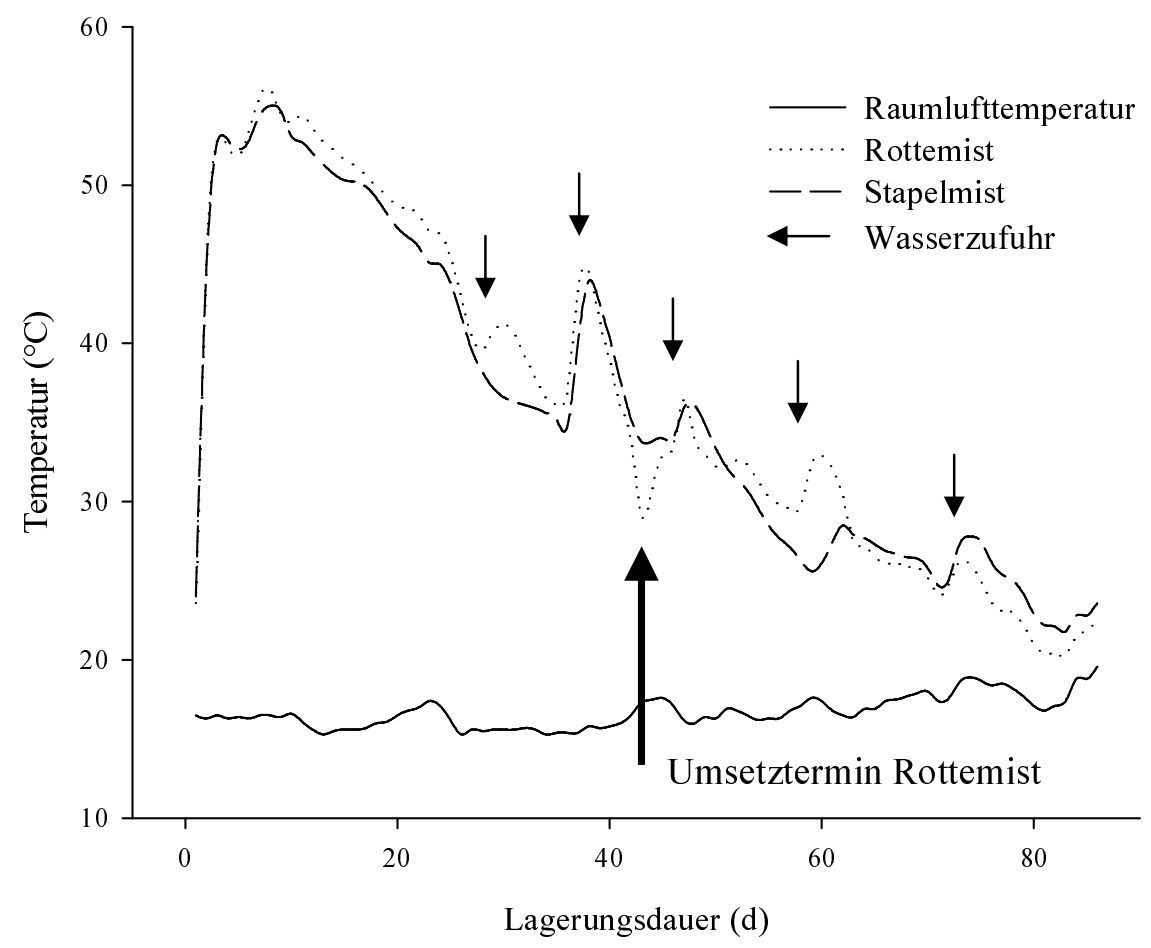

Abbildung 12: Mittlerer Temperaturverlauf $\left({ }^{\circ} \mathrm{C}\right)$ des Substrats für die Lagerungsvarianten Rottemist und Stapelmist während der dreimonatigen Lagerung, Experiment 1

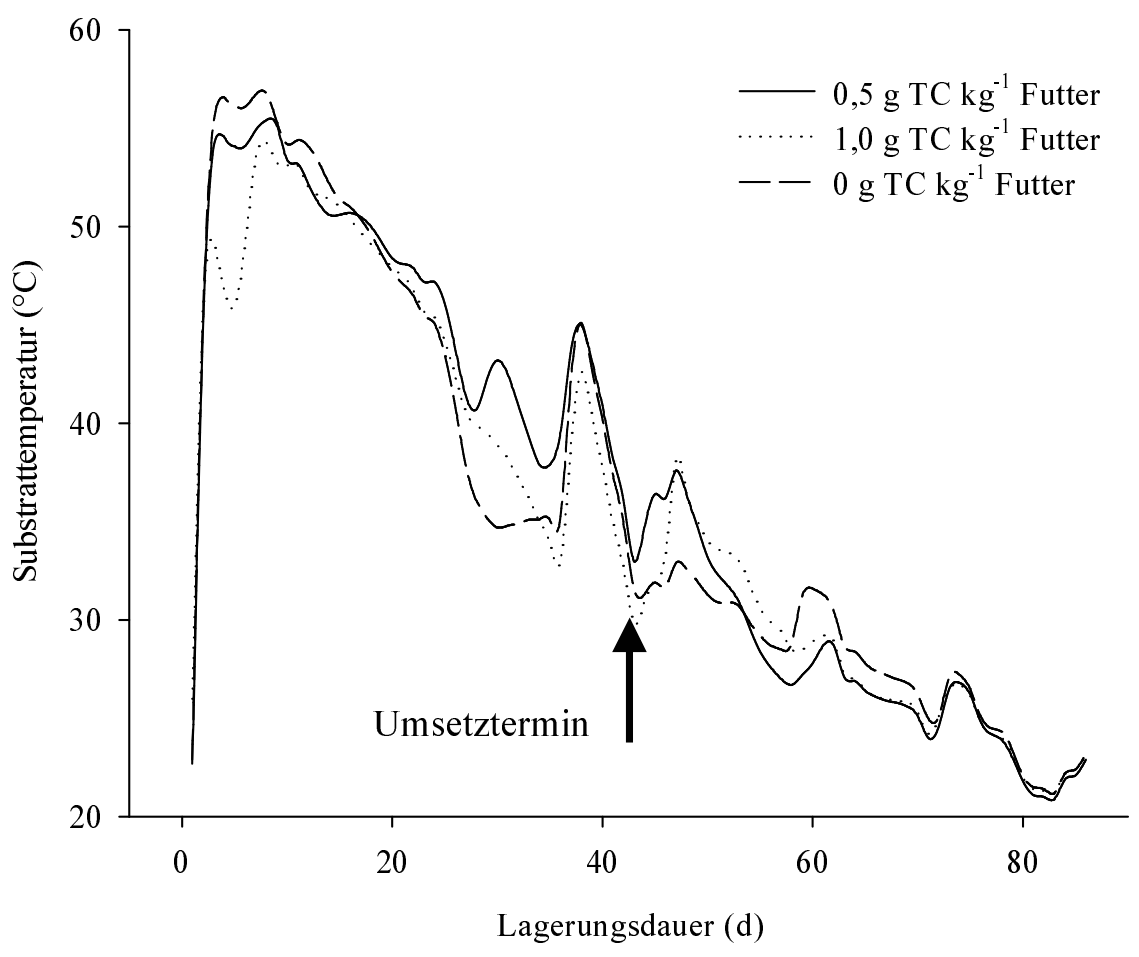

Abbildung 13: Mittlerer Temperaturverlauf $\left({ }^{\circ} \mathrm{C}\right)$ des Substrats in den Varianten mit unterschiedlicher Tetrazyklin (TC)-Konzentration während der dreimonatigen Lagerung, Experiment 1 


\section{Tetrazyklinkonzentration}

Abbildung 13 zeigt den Temperaturverlauf in Abhängigkeit von der jeweiligen TCKonzentration des Festmistes. Obwohl über die gesamte Lagerungsperiode gesehen keine signifikanten Unterschiede zwischen den einzelnen Varianten auftraten, wies die am höchsten mit TC belastete Variante vor allem zu Beginn der Lagerung die niedrigste Substrattemperatur auf, wogegen bei der unbelasteten Kontrolle die höchste Temperatur vorlag (Tukey-HSD-Test, $\mathrm{p}>0,05$ ).

Abbildung 14 veranschaulicht den Verlauf der TC-Konzentration beider Varianten über den Lagerungszeitraum im Vergleich zur mittleren Substrattemperatur während der Lagerung. Der stärkste TC-Abbau erfolgte innerhalb der ersten elf Tage. Innerhalb der ersten sechs Tage erfolgte ein Temperaturanstieg im Rottegut von $22^{\circ} \mathrm{C}$ auf $55^{\circ} \mathrm{C}$. In dieser Zeit ging die nachweisbare mittlere TC-Konzentration bei der Variante 0,5 $\mathrm{g} \mathrm{TC} \mathrm{kg}^{-1}$ Futter von 26,0 $\mathrm{mg} \mathrm{kg}^{-1}$ (RM) bzw. 44,1 $\mathrm{mg} \mathrm{kg}^{-1}$ (SM) auf 7,7 $\mathrm{mg} \mathrm{kg}^{-1}$ (RM) bzw. 11,65 mg kg-1 (SM) (ca. 70 bzw. 73 \% Abbau bei Rotte- bzw. Stapelmist) zurück.

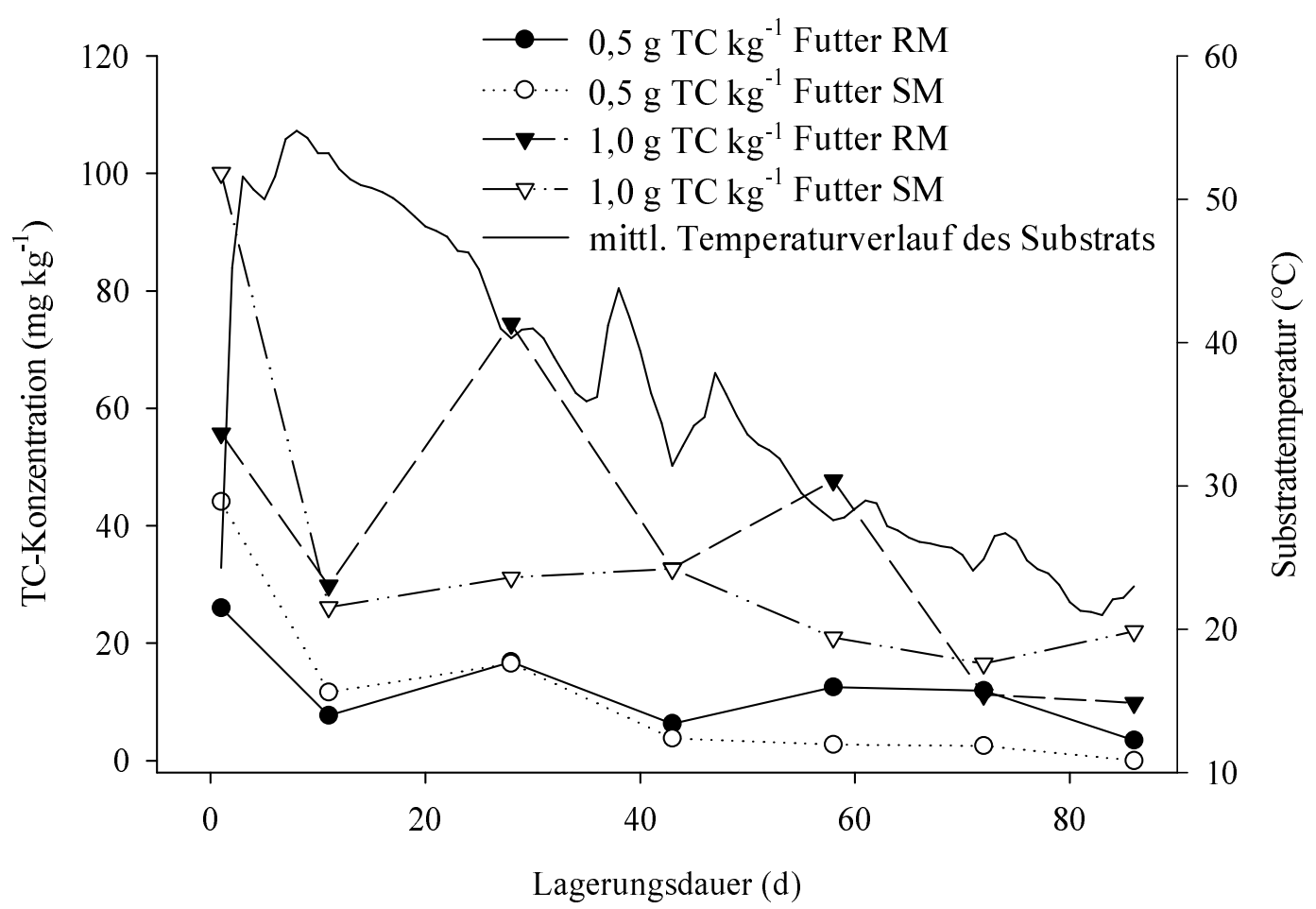

Abbildung 14: Verlauf der Konzentration an Tetrazyklin (TC) $\left(\mathrm{mg} \mathrm{kg}^{-1}\right)$ im Masthähnchenfestmist (Rotte (RM)- bzw. Stapelmist (SM)) der Varianten $0,5 \mathrm{~g} \mathrm{TC} \mathrm{kg}^{-1}$ Futter und $1,0 \mathrm{~g} \mathrm{TC} \mathrm{kg}^{-1}$ Futter über die dreimonatige Lagerung, bezogen auf Trockensubstanz (\%) 
Bei der Variante $1,0 \mathrm{~g} \mathrm{TC} \mathrm{kg}^{-1}$ Futter fiel die mittlere Konzentration während des Temperaturanstiegs von 55,7 $\mathrm{mg} \mathrm{kg}^{-1}$ (RM) bzw. 100,1 $\mathrm{mg} \mathrm{kg}^{-1}$ (SM) auf 29,8 $\mathrm{mg} \mathrm{kg}^{-1}$ (RM) bzw. 26,1 $\mathrm{mg} \mathrm{kg}^{-1}$ (SM) ab (ca. 66 bzw. $73 \%$ Abbau bei Rotte- bzw. Stapelmist). Die TC-Konzentrationen im Mist der höher dosierten Variante $\left(1,0 \mathrm{~g} \mathrm{TC} \mathrm{kg}^{-1}\right.$ Futter) überschritten erwartungsgemäß während des gesamten Untersuchungszeitraumes deutlich die Werte der niedriger dosierten Variante $\left(0,5 \mathrm{~g} \mathrm{TC} \mathrm{kg}^{-1}\right.$ Futter$)$.

Insgesamt sind am Ende der dreimonatigen Lagerungszeit für Variante $0,5 \mathrm{~g} \mathrm{TC} \mathrm{kg}^{-1}$ Futter noch $3,5 \mathrm{mg} \mathrm{kg}^{-1}$ bei Rottemist (13,3\%) und für Variante $1,0 \mathrm{~g} \mathrm{TC} \mathrm{kg}^{-1}$ Futter bei Rottemist $9,8 \mathrm{mg} \mathrm{kg}^{-1}$ (17,6\%) und bei Stapelmist 22,0 $\mathrm{mg} \mathrm{kg}^{-1}$ (22,0\%) Tetrazyklin nachweisbar (siehe Anhang, Tabelle 36). Die Stapelmistvariante 0,5 $\mathrm{g} \mathrm{TC} \mathrm{kg}^{-1}$ Futter wies nach der dreimonatigen Lagerungszeit kein Tetrazyklin oberhalb der Bestimmungsgrenze von $1 \mathrm{mg} \mathrm{kg}^{-1}$ mehr auf. Konzentrationsschwankungen wie an Tag 29 sind vermutlich auf die Probennahme zurückzuführen. Durch die Inhomogenität des Mistes konnte nicht verhindert werden, dass mit der Probe Kot und Stroh in jeweils veränderlichen Anteilen entnommen wurden. Tetrazyklin aber findet sich deutlich stärker im Kot wieder als im Stroh- bzw. Kohlenstoffanteil (siehe Kapitel 3.1.1).

Dieser Umstand wird bei der auf Kohlenstoff bezogenen Darstellungsweise deutlich (Abbildung 15). Stellt man die TC-Konzentration der Varianten bezogen auf den Kohlenstoffgehalt der Proben dar, um die unterschiedliche Probenzusammensetzung zu berücksichtigen, zeigt sich ein gleichmäßigerer Verlauf. Die Schwankungen im Konzentrationsbereich der Varianten sind geringer, nur bei der Rottemistvariante $1,0 \mathrm{mg} \mathrm{TC} \mathrm{kg}{ }^{-1}$ Futter trifft dies nicht zu. Diese zeigt weiterhin starke Schwankungen im Konzentrationsverlauf. 


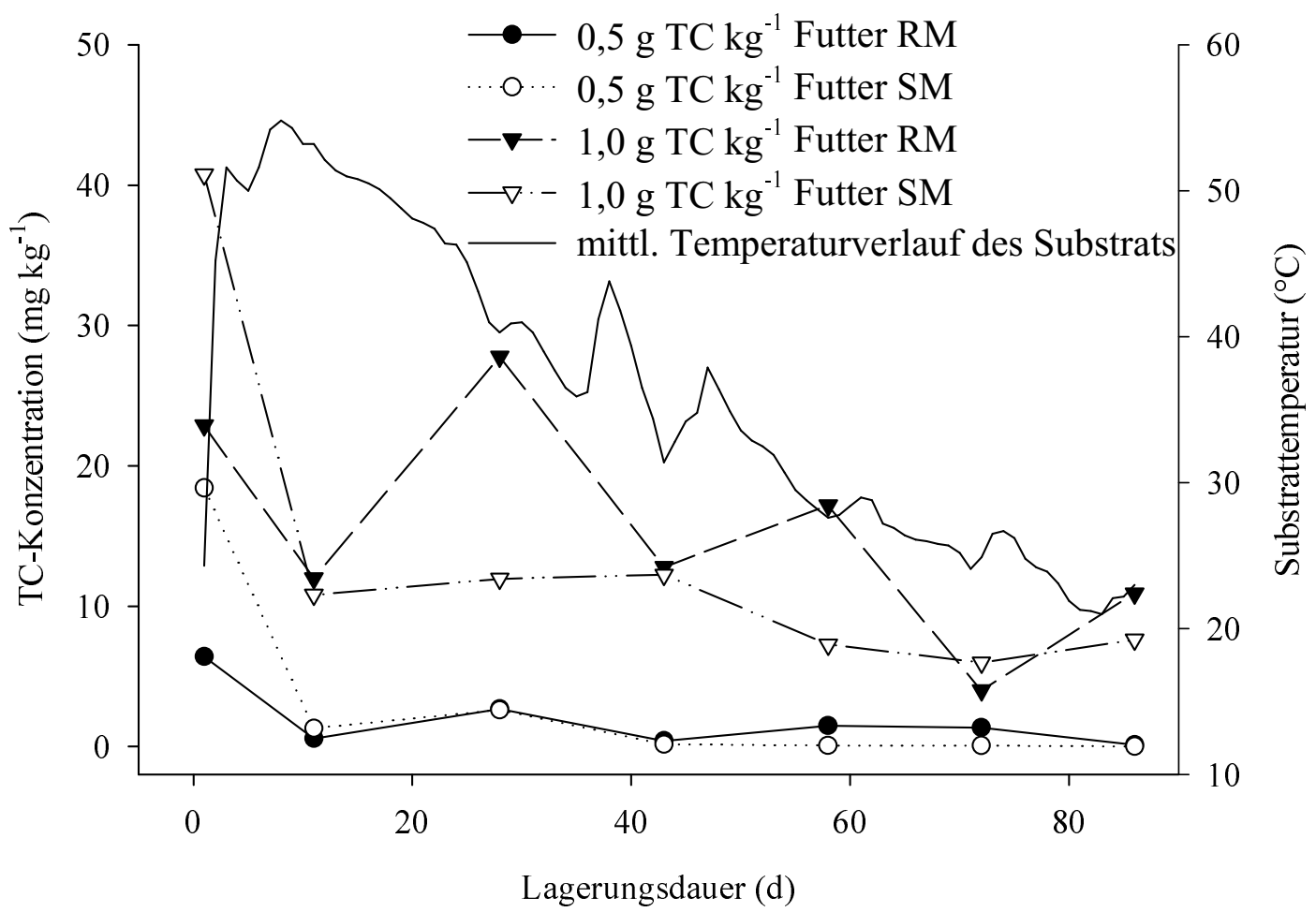

Abbildung 15: Verlauf der Konzentration an Tetrazyklin (TC) $\left(\mathrm{mg} \mathrm{kg}^{-1}\right)$ im getrockneten Masthähnchenfestmist der Varianten $0,5 \mathrm{~g} \mathrm{TC} \mathrm{kg}^{-1}$ Futter und $1,0 \mathrm{~g} \mathrm{TC} \mathrm{kg}^{-1}$ Futter (Rotte (RM)- bzw. Stapelmist (SM)) über die dreimonatige Lagerung, bezogen auf Kohlenstoff

Abbildung 16 zeigt den relativen TC-Konzentrationsverlauf der unterschiedlichen Konzentrationsstufen 0,5 g bzw. 1,0 g TC kg-1 Futter und der Lagerungsvarianten Rottebzw. Stapelmist, bezogen auf die Ausgangskonzentration. Deutlich zu erkennen ist der sehr viel gleichmäßigere Verlauf der TC-Konzentration beim Stapelmist beider Konzentrationsstufen. Nach dem starken TC-Abbau in den ersten elf Tagen nach Aufsetzen des Mistes zeigt die Kurve nur noch geringe Schwankungen. Die TCKonzentration im Rottemist dagegen weist starke Schwankungen über die gesamte Lagerungsdauer auf. In der Tendenz ist zwar ein TC-Abbau beim Rottemist zu erkennen, jedoch nicht so gleichmäßig wie beim Stapelmist.

Die Ergebnisse lassen darauf schließen, dass eine repräsentative Probennahme beim locker geschichteten Rottemist offensichtlich nicht in der Form möglich war wie beim komprimierten Stapelmist. 


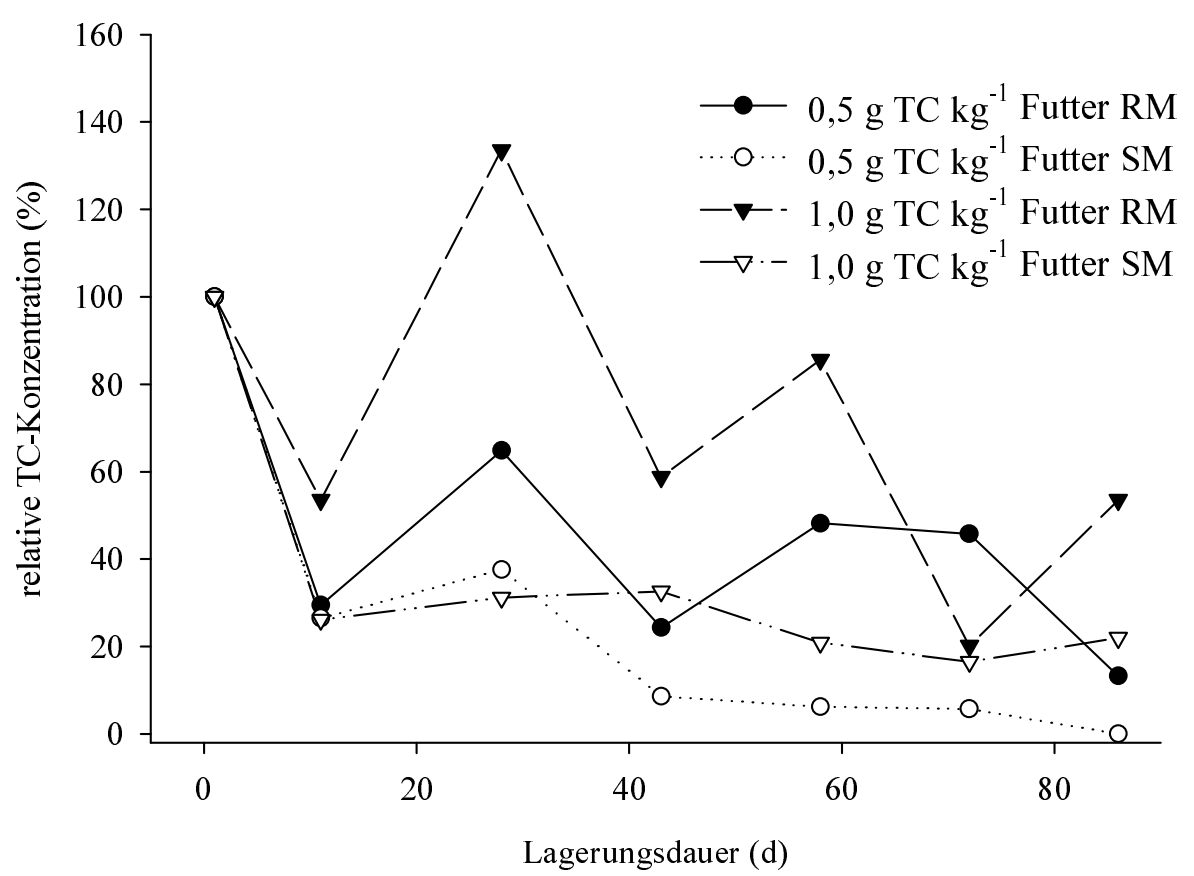

Abbildung 16: Verlauf der relativen Tetrazyklin (TC)-Konzentration (\%) bei Rotte (RM)bzw. Stapelmist (SM) über die dreimonatige Lagerung, bezogen auf die Trockensubstanz (\%)

\subsubsection{Experiment 2}

Im zweiten Experiment zur Persistenz von Tetrazyklin in Masthähnchenfestmist wurden eine einheitliche TC-Konzentration sowie die Lagerungsvarianten Rotte- bzw. Stapelmist (siehe Kapitel 3.2.2) gewählt. Die Lagerdauer war um 29 Tage kürzer als in Experiment 1. Wie in Experiment 1 wurde der Rottemist locker in die Komposter eingeschichtet, während der Stapelmist komprimiert eingelagert wurde. Tabelle 21 zeigt die Ausgangsbedingungen im Festmist zu Beginn der Lagerung die Kenngrößen nach Lagerungsende sind in

Tabelle 22 aufgeführt. Durch das Verdichten wurden etwa $10 \mathrm{~kg}$ mehr Mist in die Stapelmist-Varianten eingefüllt. Experiment 2 zeigte eine Besonderheit, da der Festmist schon eine Grundbelastung von $5,7 \mathrm{mg} \mathrm{kg}^{-1}$ Tetrazyklin aufwies. Beim ersten Probennahmetermin nach 14 Tagen Lagerdauer konnte in den Kontrollen jedoch kein TC mehr nachgewiesen werden. Da diese Konzentration deutlich unter der künstlich eingestellten Konzentration lag, waren keine hemmenden Effekte auf Mikroorganismen durch das Tetrazyklin im Festmist zu erwarten. Somit wird weiterhin von unbelasteten Kontrollen gesprochen im Gegensatz zu den behandelten Varianten. 
Tabelle 21: Stoffkenngrößen wie Stickstoff- (N), Kohlenstoff- (C), organischer Substanzgehalt (OS) des im Experiment 2 eingesetzten Masthähnchenfestmistes zu Beginn der Lagerung (in \% bezogen auf den TS-Gehalt)

\begin{tabular}{|c|c|c|c|c|c|c|c|c|}
\hline \multirow{3}{*}{$\begin{array}{l}\text { Stoffkenn- } \\
\text { größen }\end{array}$} & \multicolumn{8}{|c|}{ Lagerungsvarianten } \\
\hline & \multicolumn{2}{|c|}{ Kontrolle } & \multicolumn{3}{|c|}{ Rottemist } & \multicolumn{3}{|c|}{ Stapelmist } \\
\hline & $\mathbf{R M}$ & SM & $\mathbf{1}$ & 2 & 3 & 1 & 2 & 3 \\
\hline TS (\%) & 54,1 & 53,5 & 52,4 & 54,7 & 51,8 & 53,1 & 54,2 & 58,1 \\
\hline N (\%) & 6,3 & 6,3 & 6,3 & 6,2 & 6,2 & 6,2 & 6,2 & 6,2 \\
\hline $\mathrm{C}(\%)$ & 43,2 & 43,2 & 43,0 & 43,4 & 43,5 & 42,8 & 43,6 & 43,3 \\
\hline OS $(\%)$ & 87,5 & 87,5 & 87,7 & 88,3 & 88,1 & 88,1 & 87,7 & 87,7 \\
\hline $\mathrm{C} / \mathrm{N}$ & $6,9: 1$ & $6,9: 1$ & $6,8: 1$ & $7,0: 1$ & $7,0: 1$ & $6,9: 1$ & $7,0: 1$ & $7,0: 1$ \\
\hline $\mathrm{TC}\left(\mathrm{mg} \mathrm{kg}^{-1}\right)$ & 5,7 & 5,7 & 54,7 & 47,3 & 51,2 & 56,3 & 39,3 & 35,2 \\
\hline Mist $(\mathrm{kg})^{1}$ & 91,2 & 105,1 & 100,0 & 89,7 & 89,9 & 100,1 & 102,1 & 102,5 \\
\hline $\begin{array}{ll}1 & =\text { Frisch } \\
\mathrm{SM} & =\text { Stape } \\
\mathrm{RM} & =\text { Rotte } \\
\mathrm{TC} & =\text { Tetra }\end{array}$ & & & & & & & & \\
\hline
\end{tabular}

Tabelle 22: Stoffkenngrößen wie Stickstoff- (N), Kohlenstoff- (C), organischer Substanzgehalt (OS) des im Experiment 2 eingesetzten Masthähnchenfestmistes am Ende der Lagerung (in \% bezogen auf den TS-Gehalt)

\begin{tabular}{|c|c|c|c|c|c|c|c|c|}
\hline \multirow{3}{*}{$\begin{array}{l}\text { Stoffkenn- } \\
\text { größen }\end{array}$} & \multicolumn{8}{|c|}{ Lagerungsvarianten } \\
\hline & \multicolumn{2}{|c|}{ Kontrolle } & \multicolumn{3}{|c|}{ Rottemist } & \multicolumn{3}{|c|}{ Stapelmist } \\
\hline & $\mathbf{R M}$ & SM & 1 & 2 & 3 & 1 & 2 & 3 \\
\hline TS (\%) & 46,4 & 61,3 & 58,6 & 52,2 & 61,5 & 69,3 & 67,9 & 54,8 \\
\hline N (\%) & 6,1 & 6,7 & 5,0 & 5,7 & 5,5 & 6,6 & 7,1 & 6,1 \\
\hline $\mathrm{C}(\%)$ & 38,4 & 39,7 & 37,8 & 37,6 & 38,0 & 39,4 & 38,2 & 39,2 \\
\hline OS $(\%)$ & 81,5 & 82,6 & 76,9 & 79,1 & 79,9 & 82,0 & 82,5 & 83,4 \\
\hline $\mathrm{C} / \mathrm{N}$ & $6,3: 1$ & $5,9: 1$ & $7,5: 1$ & $6,6: 1$ & $6,9: 1$ & $6,0: 1$ & $5,4: 1$ & $6,4: 1$ \\
\hline $\mathrm{TC}\left(\mathrm{mg} \mathrm{kg}^{-1}\right)$ & 0,0 & 0,0 & 7,4 & 1,7 & 5,6 & 4,1 & 2,2 & 1,8 \\
\hline $\begin{array}{ll}\text { SM } & =\text { Stape } \\
\text { RM } & =\text { Rotte } \\
\text { TC } & =\text { Tetra }\end{array}$ & & & & & & & & \\
\hline
\end{tabular}

Der Trockensubstanzgehalt erhöhte sich bei einigen Kompostern im Verlauf der Lagerung; allerdings ist $\mathrm{zu}$ beachten, dass der Festmist kontrolliert bewässert wurde, was die für die Kompostierung notwendige Materialfeuchte gewährleisten sollte. Die Ergebnisse werden im Folgenden auf die Trockensubstanz bezogen dargestellt, um dieser externen 
Wasserzufuhr Rechnung zu tragen. Im Mittel wurden im Rottemist etwa $3 \%$ mehr organische Substanz abgebaut als im Stapelmist, und auch der Kohlenstoffanteil ging im Rottemist etwas stärker zurück. Das C:N-Verhältnis verlief bei allen Varianten parallel. Der $\mathrm{pH}-$ Wert verlief für eine Kompostierung sehr typisch: anfangs lag derpH-Wert bei ca. 5,3 im Festmist, um dann im Verlauf der Lagerung bis auf etwa 8,5 anzusteigen.

\section{Temperaturverlauf}

Abbildung 17 zeigt den mittleren Temperaturverlauf bei Rotte- und Stapelmist. Die Temperatur im Rottemist lag bis zum Umsetztermin nach vier Wochen Lagerdauer geringfügig höher als die des Stapelmistes, nach dem Umsetzen jedoch fiel die Temperatur im Rottemist steiler ab als im Stapelmist. Insgesamt lag die Temperatur im Stapelmist nach dem Umsetzen gleichmäßig auf einem höheren Niveau im Vergleich zum Rottemist und zeigte weniger Schwankungen (siehe Anhang, Tabelle 35). Ein Vergleich der Wochenmittelwerte ergab jedoch keine signifikanten Unterschiede in Bezug auf den Temperaturverlauf der jeweiligen Lagerungsart.

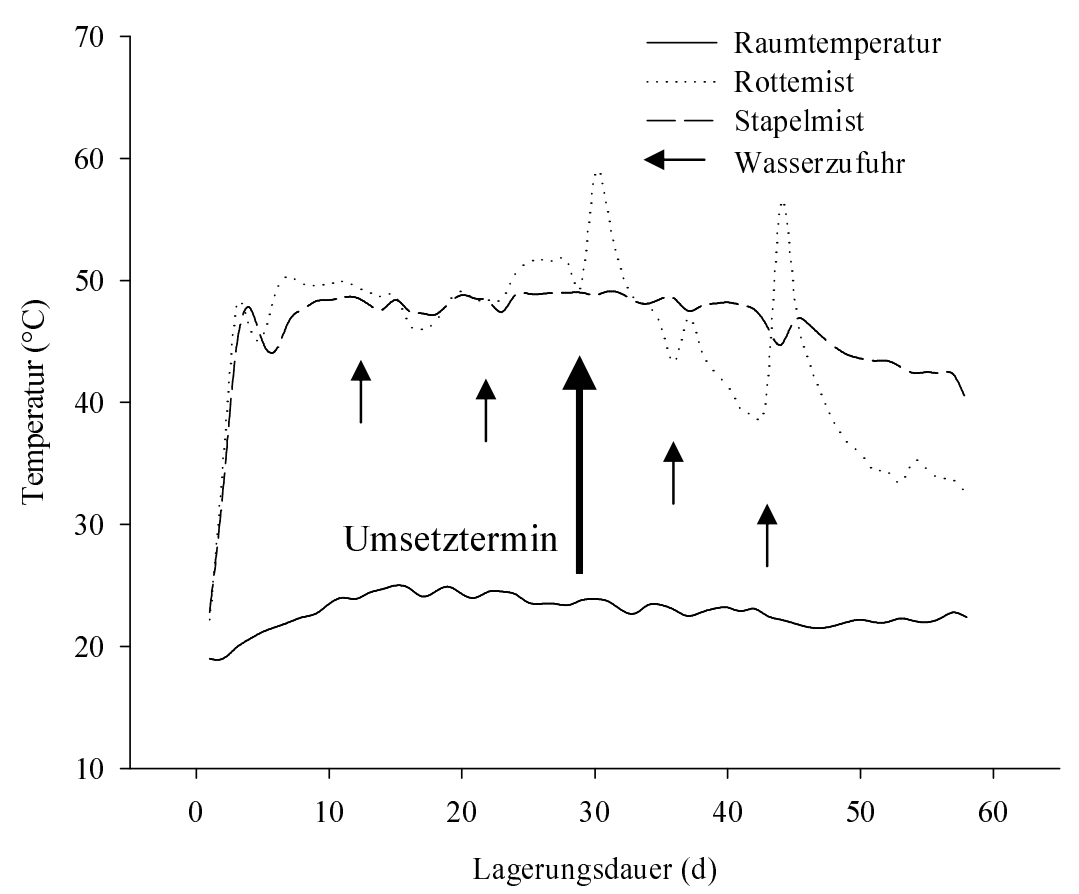

Abbildung 17: Mittlerer Temperaturverlauf $\left({ }^{\circ} \mathrm{C}\right)$ des Substrats für die Lagerungsvarianten Rottemist und Stapelmist während der zweimonatigen Lagerung, Experiment 2 


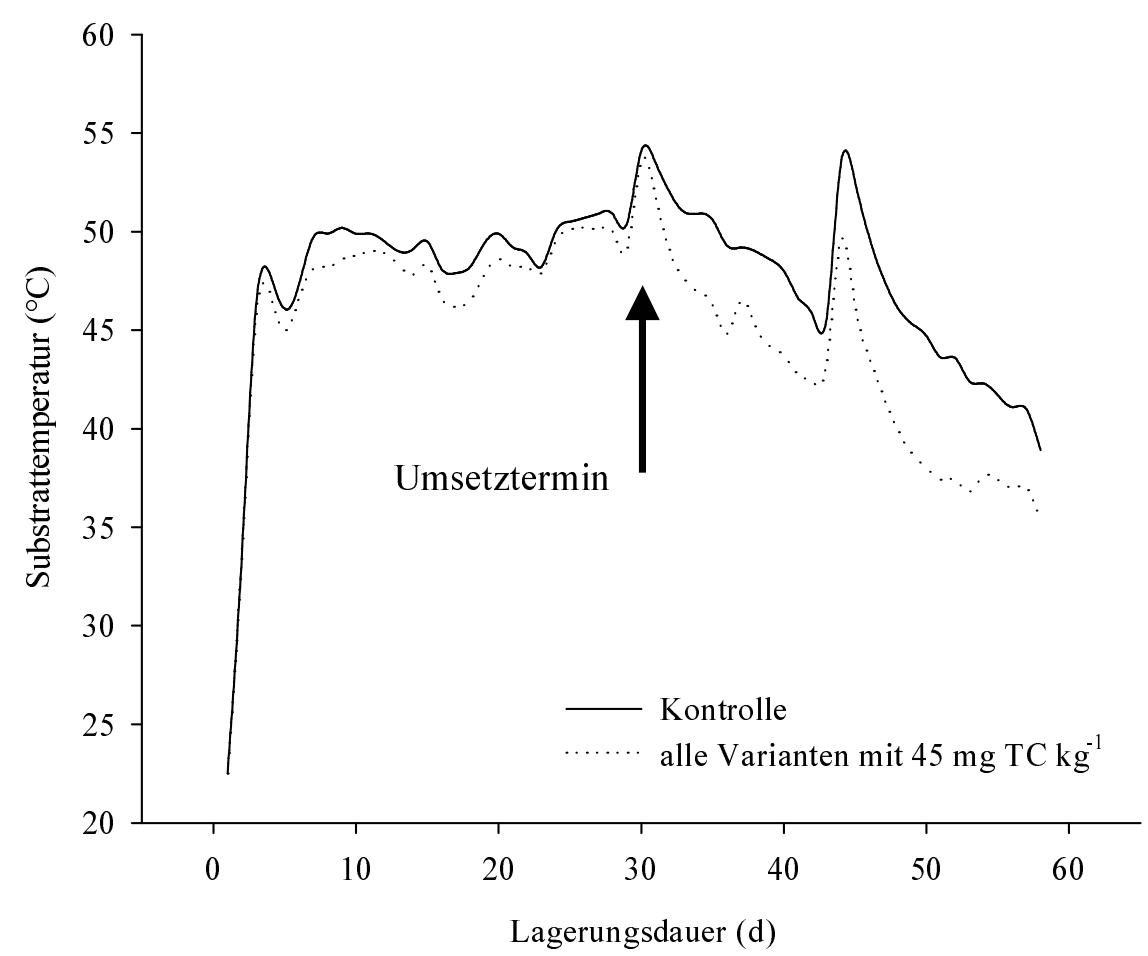

Abbildung 18: Mittlerer Temperaturverlauf $\left({ }^{\circ} \mathrm{C}\right)$ des Substrates der Rotte- und Stapelmistvarianten mit Tetrazyklin (TC)-Konzentration während der zweimonatigen Lagerung, Experiment 2

Über die gesamte Lagerungsperiode wiesen die Kontrollen ohne TC-Belastung eine höhere Temperatur auf als die Varianten mit TC (Abbildung 18). Dieser vor allem gegen Ende der Lagerung auftretende Effekt war jedoch statistisch nicht abzusichern.

\section{Tetrazyklinkonzentration}

Abbildung 19 stellt den Verlauf der relativen TC-Konzentration beider Lagerungsvarianten über den Lagerungszeitraum in Beziehung zur mittleren Lagerungstemperatur dar. Der stärkste TC-Abbau erfolgte innerhalb der ersten 14 Tage. In diesem Zeitraum erfolgte ein Temperaturanstieg von $22^{\circ} \mathrm{C}$ auf etwa $48^{\circ} \mathrm{C}$ im Festmist. 


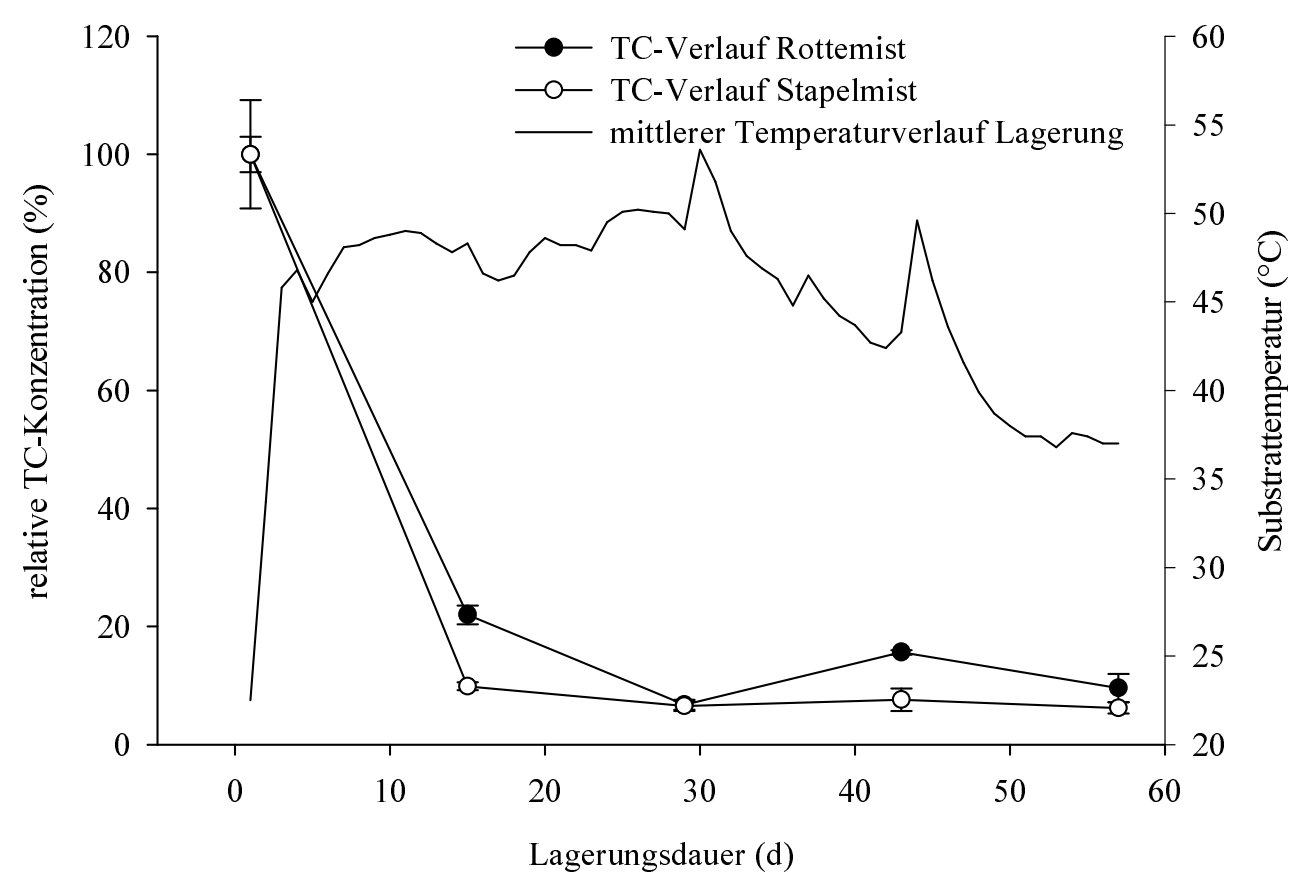

Abbildung 19: Verlauf der relativen Konzentration an Tetrazyklin (TC) (\%) (mit Standardabweichung $T$, Mittelwert aus drei Messwerten) in den Varianten Rotte- und Stapelmist über die zweimonatige Lagerung, bezogen auf Trockensubstanz (\%)

Die nachweisbare TC-Konzentration ging im Rottemist von $51,1 \mathrm{mg} \mathrm{kg}^{-1}$ auf $11,2 \mathrm{mg} \mathrm{kg}^{-1}$ zurück (ca. 78 \% Abbau); im Stapelmist betrug der Rückgang ca. $90 \%$ (von 43,6 mg kg-1 auf 4,3 mg kg-1) (siehe Anhang, Tabelle 37). Rottemist zeigte gegenüber Stapelmist keinen Unterschied im Verlauf des TC-Abbaus mit Ausnahme der schon in Experiment 1 beobachteten Schwankungen im Konzentrationsverlauf. Diese Variabilität wird besonders an Tag 43 deutlich $\left(8,0 \mathrm{mg} \mathrm{kg}^{-1}\right.$ gegenüber $3,4 \mathrm{mg} \mathrm{kg}^{-1}$ an Tag 29). Insgesamt waren am Ende der zweimonatigen Lagerungszeit im Rottemist noch durchschnittlich 4,9 mg kg ${ }^{-1} \mathrm{TC}$ (9,6\% der Ausgangskonzentration) und im Stapelmist noch im Mittel $2,7 \mathrm{mg} \mathrm{kg}^{-1} \mathrm{TC}$ (6,2 \% der Ausgangskonzentration) nachweisbar. Eine Umrechnung des TC-Gehalts auf den Kohlenstoffgehalt der Probe führte vor allem in der Rottemistvariante zu einer Glättung des Konzentrationsverlaufs (Abbildung 20). 


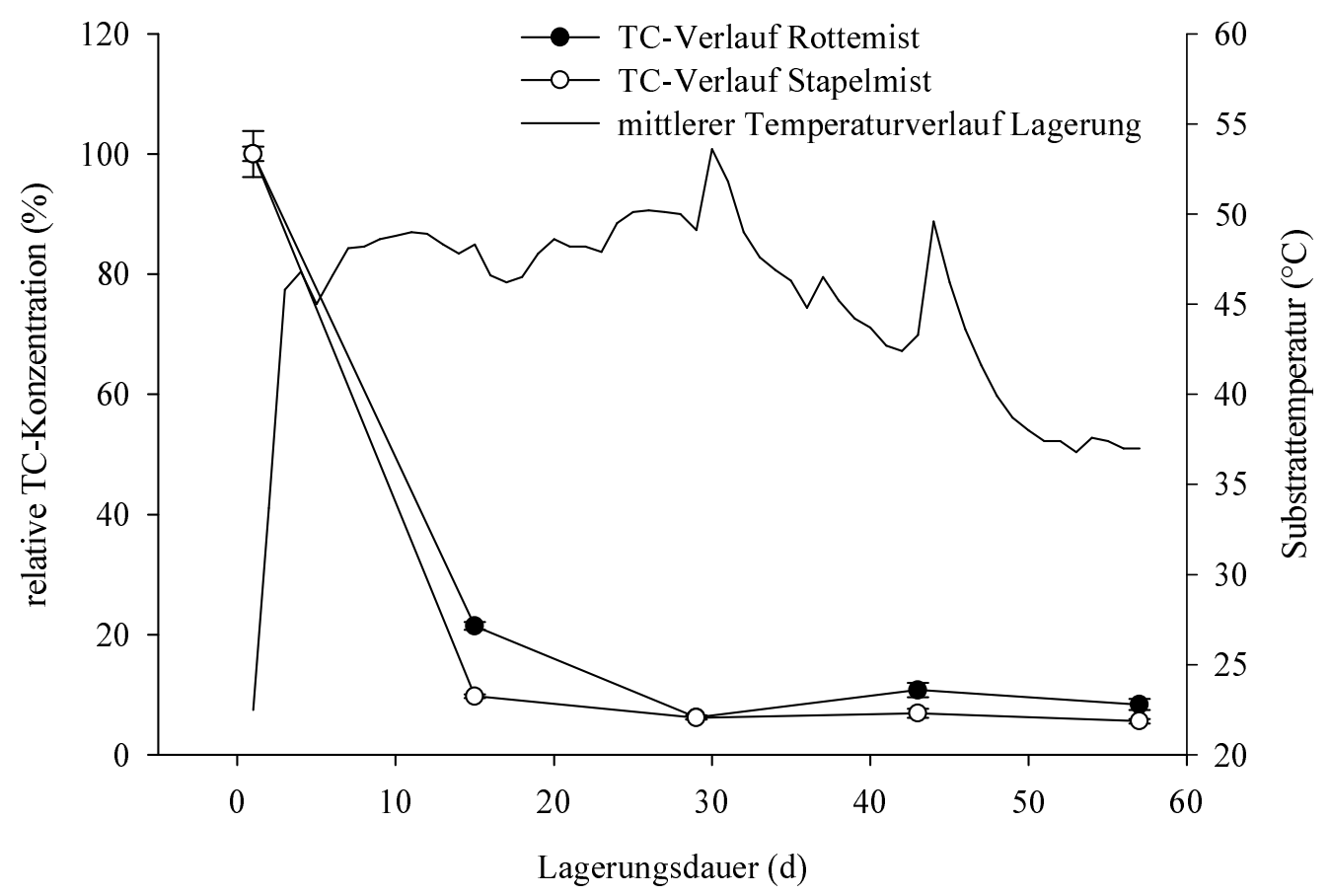

Abbildung 20: Verlauf der relativen Konzentration (mit Standardabweichung $\mathrm{T}$, Mittelwert aus drei Messwerten) an Tetrazyklin (TC) (\%) in den Varianten Rotte- und Stapelmist (bezogen auf den Trockensubstanzgehalt) über die zweimonatige Lagerung, bezogen auf Kohlenstoff

\subsection{Nachweis ausgewählter Tetrazykline und Sulfonamide in Schweinegülle}

\subsubsection{Screening anonymer Schweinegülleproben 1999/2000}

Insgesamt 168 Proben von unterschiedlichen landwirtschaftlichen Schweineproduktions betrieben wurden auf den Gehalt an drei Tetrazyklinen (Tetrazyklin, Oxy- und Chlortetrazyklin) und zwei Sulfonamiden (Sulfadiazin, Sulfamethazin) untersucht. Die Proben wurden anonym gehandhabt, es war keinerlei Zuordnung zu einzelnen Betrieben möglich. Lediglich die Stickstoff- und Trockensubstanzgehalte der Gülleproben waren bekannt (Tabelle 23).

Der mittlere TS-Gehalt der Proben betrug 4,9\% (Min: 0,5\%; Max: 14,7\%) und der mittlere N-Gehalt 0,5\% (Min: 0,1\%; Max: 1,0\%). 
Tabelle 23: Trockensubstanz- (TS) und Stickstoffgehalte (N) der Schweinegülleproben $1999 / 2000$

\begin{tabular}{lcc}
\hline Proben 1999/2000 & TS-Gehalt (\%) & N-Gehalt (\%) \\
\hline Minimum & 0,5 & 0,1 \\
Maximum & 14,7 & 1,0 \\
Mittelwert & 4,9 & 0,5 \\
Standardabweichung & 3,1 & 0,2 \\
Median & 3,8 & 0,4 \\
\hline
\end{tabular}

In etwa einem Drittel der Proben $(35,1 \%)$ waren keine Rückstände der ausgewählten Wirkstoffe oberhalb der jeweiligen Bestimmungsgrenzen nachweisbar. Eine Probe enthielt alle fünf untersuchten Antibiotika; dies entspricht $0,6 \%$ aller Proben. 1,8 \% enthielten vier der Antibiotika, in 11,9\% konnten drei Substanzen nachgewiesen werden. 23,9\% der Proben enthielten zwei Antibiotika und 26,9\% mindestens einen Wirkstoff. Die Analyseergebnisse sind in Tabelle 24 aufgelistet (siehe Anhang, Tabelle 38):

Tabelle 24: Ergebnisse des Screenings von Schweinegülleproben 1999/2000

\begin{tabular}{lc|c|cc}
\hline Wirkstoff & $\begin{array}{c}\text { Anteil in den Proben } \\
(\%)\end{array}$ & & $\begin{array}{l}\text { Zahl der Antibiotika } \\
\text { je Probe }\end{array}$ & Anteil (\%) \\
Tetrazyklin & 38,7 & 0 & 35,1 \\
Oxytetrazyklin & 0,6 & 1 & 26,8 \\
Chlortetrazyklin & 15,5 & & 23,8 \\
Sulfadiazin & 8,3 & & 3 & 11,9 \\
Sulfamethazin & 57,1 & & $4+5$ & 2,4 \\
\hline
\end{tabular}

Die am stärksten vertretenen Wirkstoffe waren Tetrazyklin und Sulfamethazin mit einem Anteil an den Gesamtproben von 38,7 bzw. 57,1\%. Sulfamethazin wies einen Median der positiven Proben von 4,3 $\mathrm{mg} \mathrm{kg}^{-1}$ und einen Maximalbefund von $62,1 \mathrm{mg} \mathrm{kg}^{-1}$ auf. Der Anteil von Sulfamethazin an den Gesamtproben lag bei 57,1\%. Sulfadiazin wies einen Median von 2,9 $\mathrm{mg} \mathrm{kg}^{-1}$ auf und hatte einen Anteil von 8,3\% der Proben. Die Minimalwerte aller untersuchten Wirkstoffe wurden durch die jeweilige Nachweisgrenze bestimmt. Tetrazyklin wies den höchsten Medianwert der positiven Proben mit 5,5 mg kg-1 auf. Oxytetrazyklin konnte lediglich in einer einzigen Probe nachgewiesen werdenin einer Konzentration von $4,8 \mathrm{mg} \mathrm{kg}^{-1}$. Für Chlortetrazyklin konnte mit einem Maximum von 
$330,7 \mathrm{mg} \mathrm{kg}^{-1}$ der höchste Einzelwert gemessen werden. Diese hohen Konzentrationen wurden jedoch nur in wenigen Proben nachgewiesen, für den größten Teil der Proben liegen die gemessenen Gehalte weit unter $80 \mathrm{mg} \mathrm{kg}^{-1}$ (Abbildung 21).

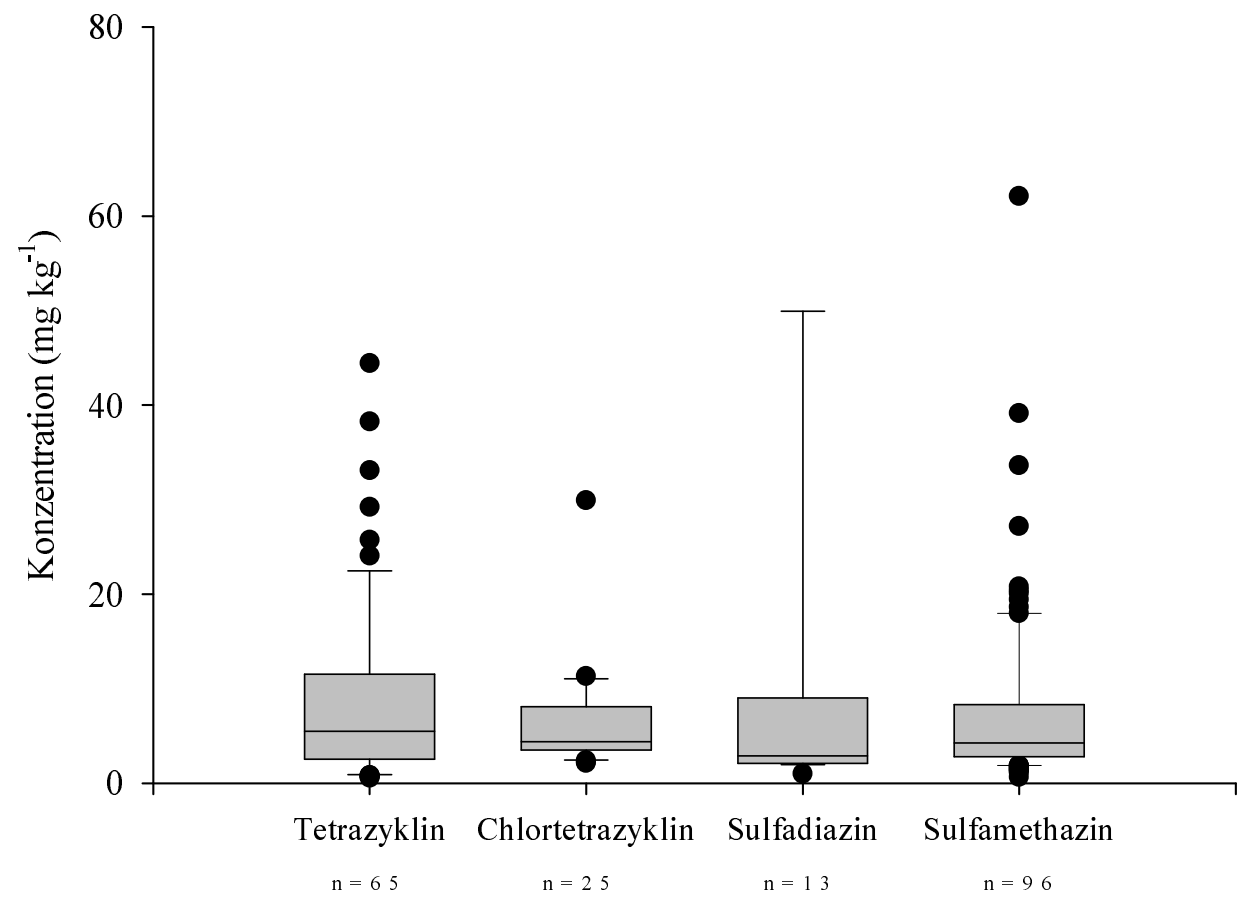

Abbildung 21: Ergebnisse des Screenings 1999/2000 für vier ausgewählte Antibiotika in Schweinegülleproben $(\mathrm{n}=168)$. Box-Whisker-Plot zeigt Median (Querstrich), $10 \%, 25 \%, 75 \%$ und $90 \%$ Perzentil mit Fehlerbalken. Die Punkte stellen die Einzelwerte außerhalb des $10 \%$ und $90 \%$ Perzentils dar. Aus Gründen der Übersichtlichkeit sind zwei Ausreißerwerte für Chlortetrazyklin (330,7 $\left.\mathrm{mg} \mathrm{kg}^{-1}\right)$ bzw. Sulfadiazin $\left(235,1 \mathrm{mg} \mathrm{kg}^{-1}\right)$ nicht dargestellt.

In den meisten Proben lagen die TS-Gehalte zwischen weniger als $3 \%$ und $6 \%$ vor. Erwartungsgemäß korrelierte der N-Gehalt stark positiv mit dem TS-Gehalt $(r=0,83)$. Mit höherem TS-Gehalt stieg der Anteil wirkstoffhaltiger Proben an. Zum Beispiel enthielten $67 \%$ der Proben mit einem TS-Gehalt zwischen 3 und $6 \%$ mindestens ein Antibiotikum, während dieser Anteil in der Klasse der Proben mit einem TS-Gehalt größer $9 \%$ schon $81 \%$ betrug (Tabelle 25). Bei Tetrazyklin als auch bei Chlortetrazyklin fiel ein Anstieg der mittleren Konzentration bis zu einem TS-Gehalt von $9 \%$ auf (von 4,3 auf $8,1 \mathrm{mg} \mathrm{kg}^{-1}$ bei TC und von 4,3 auf 6,1 $\mathrm{mg} \mathrm{kg}^{-1}$ bei CTC). Dieser Trend setzte sich ab einem TS-Gehalt der Proben von $9 \%$ jedoch nicht fort; bspw. für Tetrazyklin konnte eine mittlere Konzentration in den positiven Proben der Klasse $\geq 9 \%$ von nur noch $3,9 \mathrm{mg} \mathrm{kg}^{-1}$ 
gemessen werden. Bei Sulfadiazin zeigte sich ein deutlicherer Trend: hier stieg die mittlere Konzentration von der untersten bis zur obersten TS-Klasse von 2,1 bis auf 9,1 $\mathrm{mg} \mathrm{kg}^{-1}$ an. Für Sulfamethazin dagegen war dieser Trend nicht nachweisbar. Trotz dieser Hinweise auf eine mögliche Abhängigkeit zwischen erhöhtem TS-Gehalt und steigender Antibiotikakonzentration war für keine der untersuchten Substanzen ein signifikanter Unterschied zwischen den TS-Klassen feststellbar (Kruskall-Wallis-Test; $p>0,05$ ).

Tabelle 25: Verteilung der Schweinegülleproben und der nachgewiesenen Antibiotikain vier Trockensubstanzklassen (TS), Screening 1999/2000 (Median der positiven Proben)

\begin{tabular}{|c|c|c|c|c|}
\hline \multirow[t]{2}{*}{ Kategorie } & \multicolumn{4}{|c|}{ TS-Klassen } \\
\hline & $<3 \%$ & $\geq 3-6 \%$ & $\geq 6-9 \%$ & $\geq 9 \%$ \\
\hline Anzahl der Proben & 53 & 60 & 31 & 21 \\
\hline Anzahl der positiven Proben & 26 & 40 & 25 & 17 \\
\hline Anteil der positiven Proben (\%) & 49,1 & 66,7 & 80,6 & 81,0 \\
\hline Median Tetrazyklin $\left(\mathrm{mg} \mathrm{kg}^{-1}\right)$ & 4,3 & 6,1 & 8,1 & 3,9 \\
\hline Median Oxytetrazyklin $\left(\mathrm{mg} \mathrm{kg}^{-1}\right)$ & 4,8 & - & - & - \\
\hline Median Chlortetrazyklin (mg kg $\left.{ }^{-1}\right)$ & 4,3 & 4,0 & 6,1 & 6,0 \\
\hline Median Sulfadiazin $\left(\mathrm{mg} \mathrm{kg}^{-1}\right)$ & 2,1 & 3,5 & 4,0 & 9,1 \\
\hline Median Sulfamethazin $\left(\mathrm{mg} \mathrm{kg}^{-1}\right)$ & 5,4 & 4,0 & 3,6 & 6,7 \\
\hline$\varnothing \mathrm{TS}(\%)$ & 1,9 & 4,2 & 7,2 & 11,1 \\
\hline$\varnothing \mathrm{N}(\%)$ & 0,3 & 0,5 & 0,6 & 0,8 \\
\hline
\end{tabular}

\subsubsection{Screening anonymer Schweinegülleproben 2001/2002}

Für das Screening im Winterhalbjahr 2001/2002 standen 176 anonyme Schweinegülleproben zur Verfügung. Der durchschnittliche TS-Gehalt der Proben betrug 5,0 \% (Min: 0,7 \%; Max: 16,8 \%) und der durchschnittliche N-Gehalt 0,5 \% (Min: 0,3\%; Max: 1,1\%) (Tabelle 26). 
Tabelle 26: Trockensubstanz- (TS) und Stickstoffgehalte (N) der Schweinegülleproben $2001 / 2002$

\begin{tabular}{lcc}
\hline Proben 2001/2002 & TS-Gehalt (\%) & N-Gehalt (\%) \\
\hline Minimum & 0,7 & 0,2 \\
Maximum & 16,8 & 1,1 \\
Mittelwert & 5,0 & 0,5 \\
Standardabweichung & 2,9 & 0,2 \\
Median & 4,3 & 0,5 \\
\hline
\end{tabular}

In $12,5 \%$ der Proben waren keine Rückstände der ausgewählten Wirkstoffe oberhalb der jeweiligen Bestimmungsgrenzen nachweisbar (Tabelle 27). Zwei Proben enthielten alle fünf Antibiotika (1,1\% der Proben), 2,8\% der Proben wiesen vier Antibiotika auf. In 18,2 \% der Proben konnten mindestens drei Antibiotika nachgewiesen werden, ein Viertel der Proben allerdings enthielten zwei Wirkstoffe (25,0 \%) bzw. ein Antibiotikum (40,3 \%).

Tabelle 27: Ergebnisse des Screenings von Schweinegülleproben 2001/2002

\begin{tabular}{lc|c|lc}
\hline Wirkstoff & $\begin{array}{c}\text { Anteil in den Proben } \\
(\mathbf{\%})\end{array}$ & & $\begin{array}{l}\text { Zahl der Antibiotika } \\
\text { je Probe }\end{array}$ & Anteil (\%) \\
\cline { 1 - 2 } Tetrazyklin & 49,4 & 0 & 12,5 \\
Oxytetrazyklin & 5,1 & 1 & 40,3 \\
Chlortetrazyklin & 10,2 & 2 & 25,0 \\
Sulfadiazin & 48,9 & & 18,2 \\
Sulfamethazin & 49,3 & & $4+5$ & 3,9 \\
\hline
\end{tabular}

Neben dem häufigen Nachweis von Tetrazyklin (49,4\%) und Sulfamethazin (49,3\%) lag in diesem Probensatz auch Sulfadiazin in vergleichbarer Größenordnung vor (48,9\%) (Tabelle 27). Tetrazyklin wies den höchsten Medianwert der positiven Proben mit $7,3 \mathrm{mg} \mathrm{kg}^{-1}$ auf. Oxytetrazyklin konnte in 5,1\% der Proben mit einem Maximalwert von $136,2 \mathrm{mg} \mathrm{kg}^{-1}$ nachgewiesen werden. 10,2 \% der Proben enthielten Chlortetrazyklin mit einem Median der positiven Proben von 5,6 $\mathrm{m} \mathrm{kg}^{-1}$. Im Gegensatz dazu stehen die Sulfonamide, für die mit $3,4 \mathrm{mg} \mathrm{kg}^{-1}$ (Sulfadiazin) bzw. $3,8 \mathrm{mg} \mathrm{kg}^{-1}$ (Sulfamethazin) eine deutlich geringere mittlere Konzentration (Median der positiven Proben) ermittelt wurde (siehe Anhang, Tabelle 39). Abbildung 22 stellt die Ergebnisse grafisch dar. 


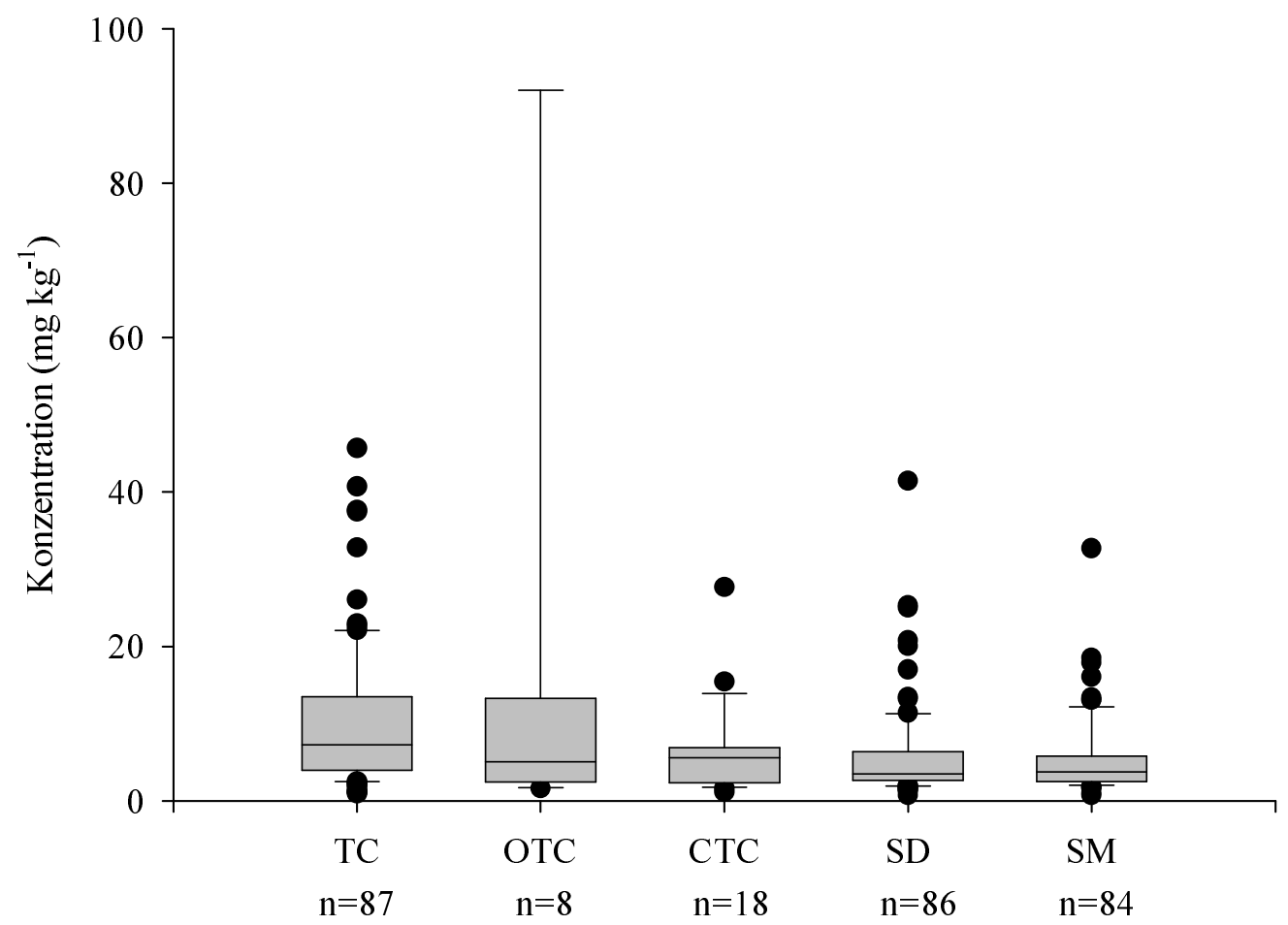

Abbildung 22: Ergebnisse des Screenings 2001/2002 für die Antibiotika Tetrazyklin (TC), Oxytetrazyklin (OTC), Chlortetrazyklin (CTC), Sulfadiazin (SD) und Sulfamethazin (SM) in Schweinegülleproben $(n=176)$. Box-Whisker-Plot zeigt Median (Querstrich), $10 \%, 25 \%, 75 \%$ und $90 \%$ Perzentil mit Fehlerbalken. Die Punkte stellen die Einzelwerte außerhalb des $10 \%$ und 90 \% Perzentils dar. Aus Gründen der Übersichtlichkeit sind zwei Ausreißerwerte für OTC (136,2 $\left.\mathrm{mg} \mathrm{kg}^{-1}\right)$ bzw. SM (167,0 $\left.\mathrm{mg} \mathrm{kg}^{-1}\right)$ nicht dargestellt.

Auch im Probennahmejahr 2001/2002 lag der TS-Gehalt in der überwiegenden Zahl der Proben zwischen weniger als 3 bis $6 \%$. In diesen beiden TS-Klassen befanden sich 38 bzw. 61 der mit mindestens einem Antibiotikum bzw. zwei Wirkstoffen belasteten Proben (76,0 bzw. 89,7\%). Im Bereich ab $9 \%$ TS-Gehalt enthielten immerhin noch $85 \%$ der Proben dieser Klasse mindestens ein Antibiotikum (Tabelle 28). Erwartungsgemäß korrelierte der N-Gehalt wieder stark positiv mit dem TS-Gehalt $(r=0,77)$.

Bei Tetrazyklin fiel ein Anstieg der mittleren Konzentration bis zu einem TS-Gehalt von $9 \%$ auf (von 3,8 auf 9,2 $\mathrm{mg} \mathrm{kg}^{-1}$ ). Dieser Trend setzte sich ab einem TS-Gehalt der Proben von $9 \%$ jedoch nicht fort; für Tetrazyklin konnte nur noch eine mittlere Konzentration in den positiven Proben der Klasse größer $9 \%$ TS von $8,6 \mathrm{mg} \mathrm{kg}^{-1}$ gemessen werden. Die mittlere Konzentration (Median) von Chlortetrazyklin dagegen stieg mit jeder TS-Klasse an; von 1,6 bei unter $3 \%$ TS bis zu 10,4 $\mathrm{mg} \mathrm{kg}^{-1}$ bei einem TS-Gehalt von über $9 \%$ in den Proben. Bei Sulfadiazin erhöhte sich die mittlere Konzentration mit steigendem TS-Gehalt 
der Proben von 2,8 auf 5,9 $\mathrm{mg} \mathrm{kg}^{-1}$. Für Sulfamethazin und Oxytetrazyklin war dieser Trend nicht nachweisbar, die mittleren Konzentrationen unterschieden sich zwischen den TS-Klassen kaum.

Tabelle 28: Verteilung der Schweinegülleproben und der nachgewiesenen Antibiotikain vier Trockensubstanzklassen (TS), Screening 2001/2002 (Median der positiven Proben)

\begin{tabular}{|c|c|c|c|c|}
\hline \multirow[t]{2}{*}{ Kategorie } & \multicolumn{4}{|c|}{ TS-Klassen } \\
\hline & $<3 \%$ & $\geq 3-6 \%$ & $\geq 6-9 \%$ & $\geq 9 \%$ \\
\hline Anzahl der Proben & 50 & 68 & 36 & 20 \\
\hline Anzahl d. positiven Proben & 38 & 61 & 36 & 17 \\
\hline Anteil d. positiven Proben $(\%)$ & 76,0 & 89,7 & 100,0 & 85,0 \\
\hline Median Tetrazyklin $\left(\mathrm{mg} \mathrm{kg}^{-1}\right)$ & 3,8 & 6,9 & 9,2 & 8,6 \\
\hline Median Oxytetrazyklin $\left(\mathrm{mg} \mathrm{kg}^{-1}\right)$ & - & 14,8 & 2,6 & 6,6 \\
\hline Median Chlortetrazyklin ( $\left.\mathrm{mg} \mathrm{kg}^{-1}\right)$ & 1,6 & 5,4 & 6,2 & 10,4 \\
\hline Median Sulfadiazin ( $\left.\mathrm{mg} \mathrm{kg}^{-1}\right)$ & 2,8 & 3,5 & 3,5 & 5,9 \\
\hline Median Sulfamethazin $\left(\mathrm{mg} \mathrm{kg}^{-1}\right)$ & 3,0 & 3,7 & 3,9 & 3,7 \\
\hline$\varnothing \mathrm{TS}(\%)$ & 2,0 & 4,4 & 7,1 & 10,9 \\
\hline$\varnothing \mathrm{N}(\%)$ & 0,3 & 0,5 & 0,6 & 0,8 \\
\hline
\end{tabular}

Diese Ergebnisse ließen sich statistisch absichern: für Tetrazyklin, Chlortetrazyklin und Sulfadiazin war ein signifikanter Unterschied zwischen den TS-Klassen feststellbar (Kruskall-Wallis-Test; $\mathrm{p}<0,05$ ). Die mittlere Konzentration von Tetrazyklin (Median) der TS-Klasse unter $3 \%$ TS unterschied sich signifikant von den restlichen drei TS-Klassen ( $\mathrm{p} \leq 0,01$ bei den TS-Klassen unter $3 \%$ und bis $6 \% ; \mathrm{p} \leq 0,001$ bei den TS-Klassen unter $3 \%$ und ab $6 \%$ bis über $9 \%$ TS-Gehalt), bei Chlortetrazyklin bestand ein statistisch abgesicherter Unterschied zwischen der TS-Klasse von unter $3 \%$ bis unter $9 \%(\mathrm{p} \leq 0,05)$ (Tabelle 29).

Für die mittlere Konzentration von Sulfadiazin in den Proben bestand ein signifikanter Unterschied lediglich zwischen der TS-Klasse unter $3 \%$ TS und über $9 \%$ TS ( $\leq \leq 0,01)$. Entsprechend der Signifikanzniveaus von $\mathrm{p} \leq 0,05, \mathrm{p} \leq 0,01$ und $\mathrm{p} \leq 0,001$ wurden ein bis drei Sternchen vergeben. 
Tabelle 29: Signifikante Effekte zwischen den einzelnen Trockensubstanz (TS)-Klassen der Schweinegülleproben 2001/2002 bezüglich verschiedener Antibiotika (Mann-Whitney-U-Test)

\begin{tabular}{|c|c|c|c|c|c|c|}
\hline \multirow[t]{2}{*}{ Antibiotika } & \multicolumn{6}{|c|}{ Gruppenkombinationen der TS-Klassen } \\
\hline & $1+2$ & $1+3$ & $1+4$ & $2+3$ & $2+4$ & $3+4$ \\
\hline $\mathrm{TC}$ & $* *$ & $* * *$ & $* * *$ & 0,406 & 0,095 & 0,539 \\
\hline CTC & $*$ & $*$ & 0,077 & 0,228 & 0,439 & 0,795 \\
\hline SD & 0,062 & 0,169 & $* *$ & 0,916 & 0,104 & 0,244 \\
\hline
\end{tabular}

\subsection{Modellversuche zur Mobilität von Tetrazyklin und Sulfadiazin in Böden}

\subsubsection{Verlagerbarkeit im Kick-Brauckmann-Gefäß}

Bei der Analyse dieser Sickerwasserproben stand aufgrund der Versuchsanstellung je Fraktion nur ein Probevolumen von $50 \mathrm{ml}$ zur Verfügung. Daraus ergab sich ein Anreicherungsfaktor von 1:25 und eine Anhebung der Bestimmungsgrenze auf $2 \mu \mathrm{g} 1^{-1}$ bei Tetrazyklin und $6 \mu \mathrm{g} \mathrm{l}^{-1}$ bei Sulfadiazin (siehe Kapitel 3.1.3).

\subsubsection{Starkregensimulation nach 48 Stunden}

\section{Sickerwasser}

In den unter humosem Sandboden sowie lehmigem Schluff gewonnenen Wasserproben konnten weder Tetrazyklin noch Sulfadiazin oberhalb der Bestimmungsgrenze von 2 bzw. $6 \mu \mathrm{g}^{-1}$ nachgewiesen werden. Dagegen wies das Sickerwasser unter Nullerde bei zwei Gefäßen Tetrazyklin in Konzentrationen zwischen 2 und $10 \mu \mathrm{g} 1^{-1}$ auf (Abbildung 23). Sulfadiazin war ebenfalls nicht nachweisbar. 


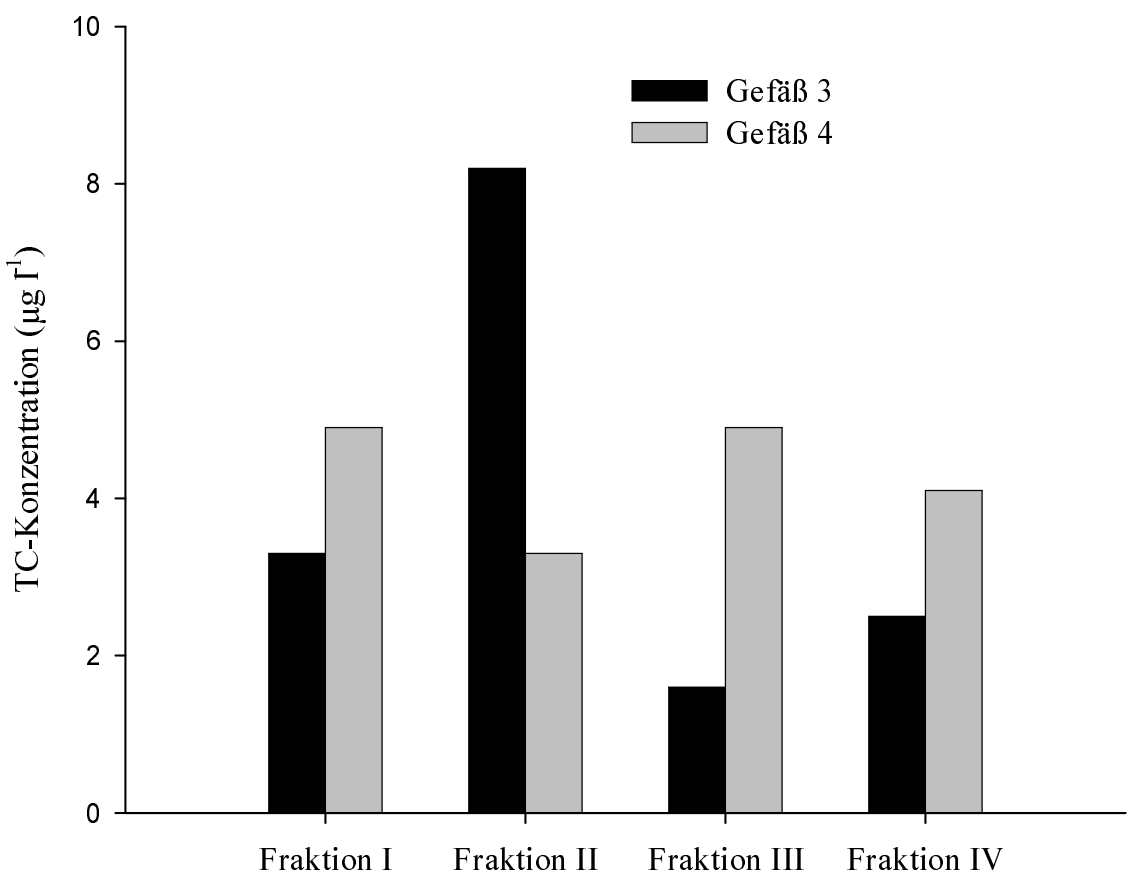

Abbildung 23: Tetrazyklin (TC)-Konzentrationen in den Sickerwasserfraktionen HIV (je Fraktion $100 \mathrm{ml}$ ) der Variante Nullerde/Torf, Starkregenereignis 48 Stunden nach Gülleaufbringung (Gefäß 2 aufgrund Negativbefund nicht dargestellt)

Die in Tabelle 30 aufgelisteten Ergebnisse (um die Wiederfindung korrigiert) wurden durch Untersuchungen des Referenzlabors (Zentrumsabteilung für Lebensmitteltechnologie und -toxikologie der Tierärztlichen Hochschule Hannover) in der Größenordnung bestätigt (siehe Anhang, Tabelle 40). 
Tabelle 30: Gehalte an Tetrazyklin (TC) und Sulfadiazin (SD) $\left(\mu \mathrm{g}^{-1}\right)$ im Sickerwasser (je Fraktion $100 \mathrm{ml}$ ) bei Beregnung 48 Stunden nach Gülleaufbringung

\begin{tabular}{lllllllll}
\hline Varianten & \multicolumn{2}{c}{ 1. Fraktion } & \multicolumn{2}{c}{ 2. Faktion } & \multicolumn{2}{c}{ 3. Fraktion } & \multicolumn{2}{c}{ 4. Fraktion } \\
& TC & SD & TC & SD & TC & SD & TC & SD \\
\hline Gefäß 1 (KT) & n.n. & n.n. & n.n. & n.n. & n.n. & n.n. & n.n. & n.n. \\
Gefäß 2 & n.n. & n.n. & n.n. & n.n. & n.n. & n.n. & n.n. & n.n. \\
Gefäß 3 & n.n. & n.n. & n.n. & n.n. & n.n. & n.n. & n.n. & n.n. \\
Gefäß 4 & n.n. & n.n. & n.n. & n.n. & n.n. & n.n. & n.n. & n.n. \\
\hline & & & Lehmiger Schluff & & & \\
\hline Gefäß 1 (KT) & n.n. & n.n. & n.n. & n.n. & n.n. & n.n. & n.n. & n.n. \\
Gefäß 2 & n.n. & n.n. & n.n. & n.n. & n.n. & n.n. & n.n. & n.n. \\
Gefäß 3 & n.n. & n.n. & n.n. & n.n. & n.n. & n.n. & n.n. & n.n. \\
Gefäß 4 & n.n. & n.n. & n.n. & n.n. & n.n. & n.n. & n.n. & n.n. \\
\hline & & & \multicolumn{2}{c}{ Nullerde/ Torf } & & & & \\
\hline Gefäß 1 (KT) & n.n. & n.n. & n.n. & n.n. & n.n. & n.n. & n.n. & n.n. \\
Gefäß 2 & n.n. & n.n. & n.n. & n.n. & n.n. & n.n. & n.n. & n.n. \\
Gefäß 3 & 3,3 & n.n. & 8,2 & n.n. & 1,6 & n.n. & 2,5 & n.n. \\
Gefäß 4 & 4,9 & n.n. & 3,3 & n.n. & 4,9 & n.n. & 4,1 & n.n. \\
\hline
\end{tabular}

kursiv: Wert unterhalb Bestimmungsgrenze von $2 \mu g t^{l}$, durch Referenzlabor bestätigt n.n.: nicht nachweisbar

$\mathrm{KT}=$ Kontrolle

$\mathrm{TC}=$ Tetrazyklin; $\mathrm{SD}=$ Sulfadiazin

\section{Boden}

Die Analyse der Bodenproben der Kontrollvarianten, also der weder mit Gülle noch mit Wirkstoff behandelten Gefäße, ergab keine Tetrazyklin- und Sulfadiazinkonzentration oberhalb der Bestimmungsgrenze von $1 \mu \mathrm{g} \mathrm{kg}^{-1}$ Boden (Nachweis durch Referenzlabor). Bei gleicher aufgebrachter Wirkstoffmenge lagen nach Starkregensimulation 48 Stunden nach Gülleaufbringung deutlich unterschiedliche TC-Gehalte in den verschiedenen Böden vor (Abbildung 24). Aus technischen Gründen konnte das Ergebnis der Bodenprobe aus Gefäß 2, lehmiger Schluff, nicht in die Auswertung eingehen.

Während Nullerde die geringsten Werte mit durchschnittlich $287 \mu \mathrm{g} \mathrm{kg}^{-1}$ im Ober- und $160 \mu \mathrm{g} \mathrm{kg}^{-1}$ im Unterboden aufwies, wurden die höchsten Konzentrationen beim lehmigen Schluff mit durchschnittlich $8773 \mu \mathrm{g} \mathrm{kg}^{-1}$ im Ober- und $1505 \mu \mathrm{g} \mathrm{kg}^{-1}$ im Unterboden gefunden; der humose Sandboden nahm eine Mittelstellung ein (Ø Oberboden: 
$2898 \mu \mathrm{g} \mathrm{kg}^{-1}$; Ø Unterboden: $621 \mu \mathrm{g} \mathrm{kg}^{-1}$ ) (siehe Anhang, Tabelle 41). Aber auch innerhalb der Bodenarten sind hohe Konzentrationsschwankungen $\mathrm{zu}$ verzeichnen. Am stärksten ist dies beim humosen Sandboden der Fall; hier reicht im Oberboden die Spanne von $1757 \mu \mathrm{g} \mathrm{kg}^{-1}$ bis $2190 \mu \mathrm{g} \mathrm{kg}^{-1}$ und im Unterboden von $238 \mu \mathrm{g} \mathrm{kg}^{-1}$ bis $1338 \mu \mathrm{g} \mathrm{kg}^{-1}$.

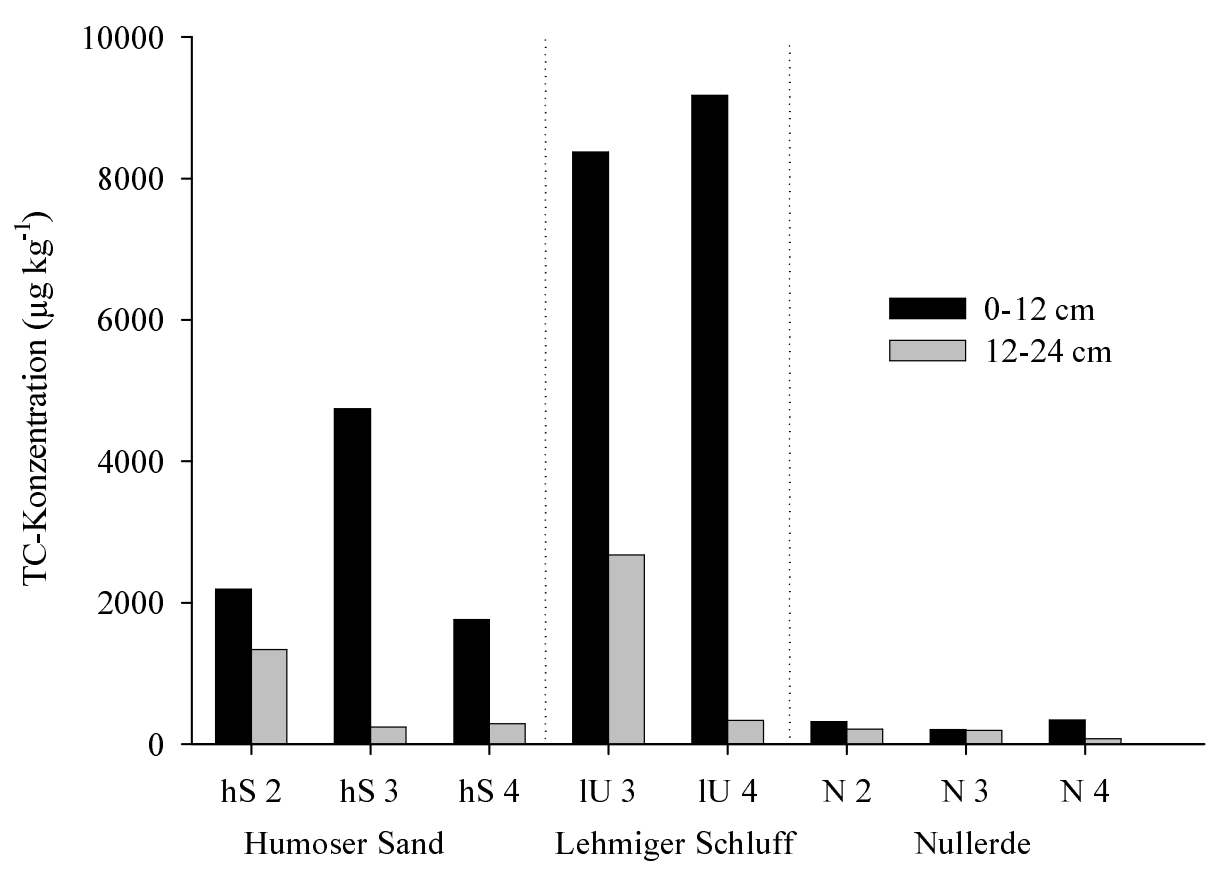

Abbildung 24: Mittlere Tetrazyklin (TC)-Konzentration in den oberen 0-12 cm und den unteren 12-24 cm der Versuchsböden (hS = humoser Sand; IU = lehmiger Schluff; N = Nullerde) je Gefäß; Starkregenereignis 48 Stunden nach Gülleaufbringung (Gefäß IU 2 ist nicht dargestellt)

Sulfadiazin wurde im Oberboden in Konzentrationen von $18 \mu \mathrm{g} \mathrm{kg}^{-1}$ (Nullerde/ Torf) bis über $330 \mu \mathrm{g} \mathrm{kg}^{-1}$ (humoser Sand) nachgewiesen (Abbildung 25). Anders als bei Tetrazyklin ließ sich bei Sulfadiazin im humosen Sandboden deutlich mehr Wirkstoff nachweisen (Ø $208 \mu \mathrm{g} \mathrm{kg}^{-1}$ ) als im lehmigen Schluff $\left(\varnothing 66 \mu \mathrm{g} \mathrm{kg}^{-1}\right)$ oder im Torfboden, wo die geringste Sulfadiazinkonzentration gemessen werden konnte (Ø $28 \mu \mathrm{g} \mathrm{kg}^{-1}$ ) (siehe Anhang, Tabelle 42). Insgesamt liegen die Sulfadiazingehalte jedoch in allen Böden trotz vergleichbarer Dosierung deutlich unter den Tetrazyklingehalten. Für Sulfadiazin wurde nur eine geringe Verlagerungswahrscheinlichkeit angenommen, daher entfiel die Probennahme in Bezug auf den Unterboden. 


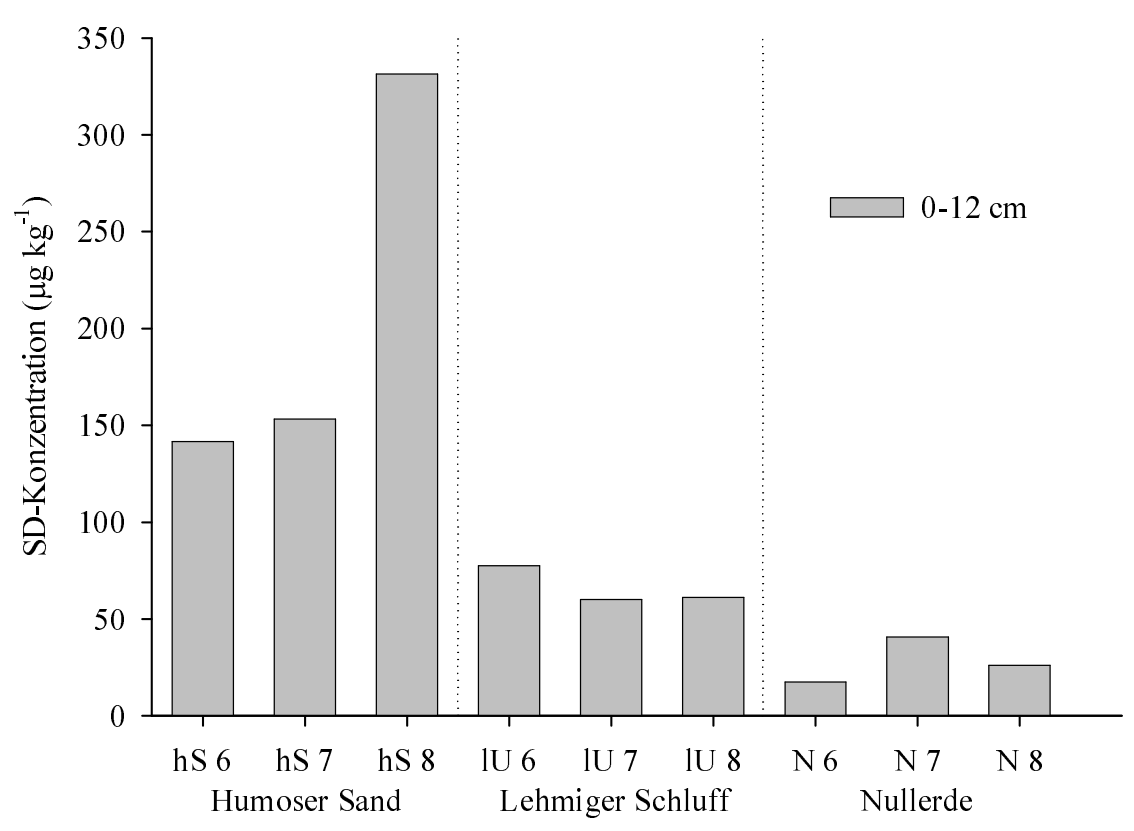

Abbildung 25: Mittlere Sulfadiazin (SD)-Konzentration in den oberen 0-12 cm der Versuchsböden ( $\mathrm{hS}=$ humoser Sand; $1 \mathrm{U}=$ lehmiger Schluff; $\mathrm{N}=$ Nullerde) je Gefäß; Starkregenereignis 48 Stunden nach Gülleaufbringung

\subsubsection{Starkregensimulation nach 10 Wochen}

\section{Sickerwasser}

Zehn Wochen nach Versuchsansatz wurde wie oben beschrieben bei den verbleibenden 24 Gefäßen eine Beregnung durchgeführt und das Sickerwasser fraktioniert aufgefangen. In allen untersuchten Sickerwasserfraktionen konnten weder Tetrazyklin noch Sulfadiazinkonzentrationen oberhalb der Bestimmungsgrenze von 2 bzw. $6 \mu \mathrm{g} \mathrm{l}^{-1}$ nachgewiesen werden.

\section{Boden}

Im Vergleich zur Beregnung 48 Stunden nach Versuchsansatz war nach zehn Wochen ein deutlicher Konzentrationsrückgang in den Antibiotikagehalten der Böden zu verzeichnen. So wies der humose Sand im Oberboden mit durchschnittlich $553 \mu \mathrm{g} \mathrm{kg}^{-1}$ Tetrazyklin ca. $20 \%$ der früher ermittelten Konzentration auf (Abbildung 26). Noch stärker fiel der Konzentrationsrückgang beim Oberboden des lehmigen Schluffs aus: hier waren nach zehn Wochen nur noch durchschnittlich 1,5\% TC (128 $\left.\mathrm{g} \mathrm{kg}^{-1}\right)$ nachweisbar. Dieser Effekt war für Tetrazyklin ausgeprägter als für Sulfadiazin (Abbildung 27). Letzteres konnte aber 
bereits nach 48 Stunden nur in sehr viel geringerer Konzentration im Vergleich zu TC nachgewiesen werden.

Tetrazyklin zeigte weiterhin starke Konzentrationsschwankungen innerhalb der Bälen, besonders im Oberboden (z.B. lehmiger Schluff: von $17 \mu \mathrm{g} \mathrm{kg}^{-1}$ bis zu $340 \mu \mathrm{g} \mathrm{kg}^{-1}$ ). Diese Schwankungen traten aber auch im Unterboden auf, bspw. beim Torfboden. Hier wies das Gefäß 12 nach zehn Wochen kein Tetrazyklin auf, das Gefäß 11 dagegen $81 \mu \mathrm{g} \mathrm{kg}^{-1}$. Es waren jedoch weiterhin höhere TC-Gehalte im Ober- als im Unterboden zu verzeichnen; das Verhältnis der Konzentrationen in Ober- und Unterboden blieb nahezu gleich (siehe Anhang, Tabelle 41).

Die mittleren Sulfadiazingehalte lagen nach zehn Wochen deutlich unter denen, die 48 Stunden nach Versuchsansatz ermittelt wurden. Im humosen Sand konnten etwa $15 \%$ und im lehmigen Schluff 19\% gemessen werden. Am geringsten war der Rückgang der Sulfadiazingehalte im Torfboden; hier waren nach zehn Wochen noch $77 \%$ im Boden nachweisbar (siehe Anhang, Tabelle 42).

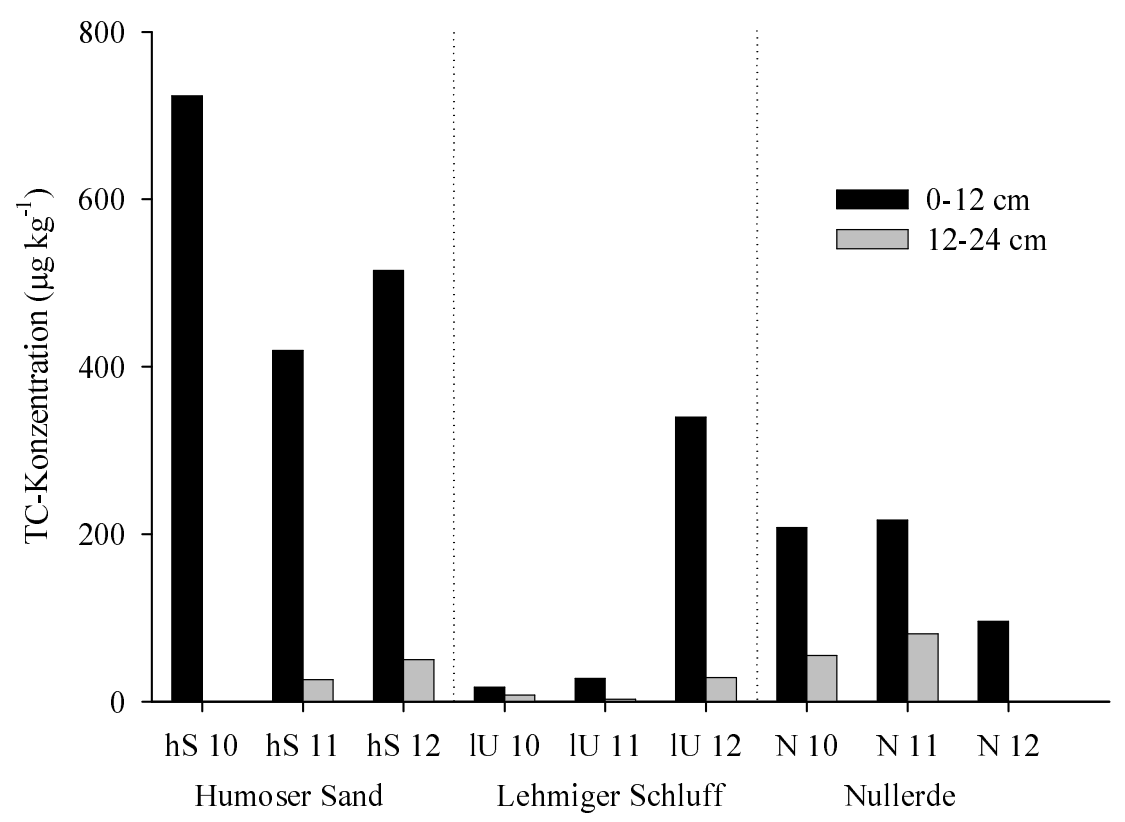

Abbildung 26: Mittlere Tetrazyklin (TC)-Konzentration in den oberen 0-12 cm und den unteren 12-24 cm der Versuchsböden (hS = humoser Sand; $1 \mathrm{U}=$ lehmiger Schluff; N = Nullerde) je Gefäß; Starkregenereignis zehn Wochen nach Gülleaufbringung 


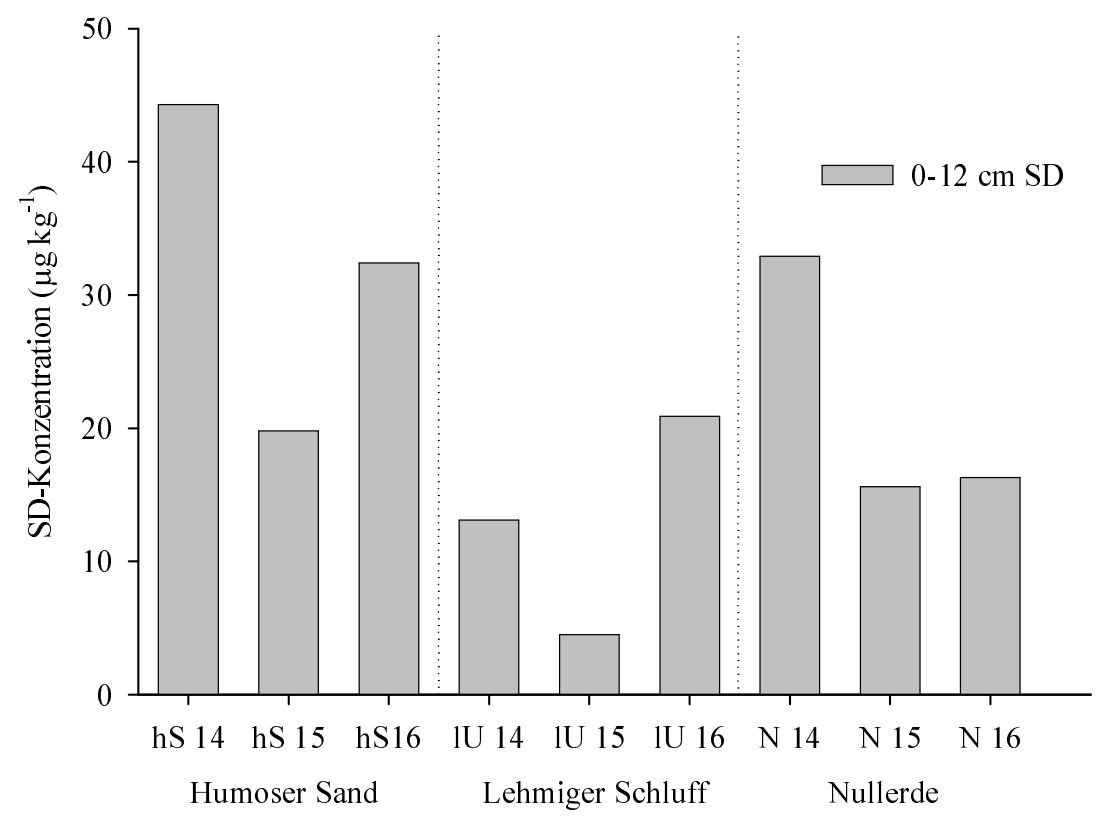

Abbildung 27: Mittlere Sulfadiazin (SD)-Konzentration in den oberen 0-12 cm der Versuchsböden (hS = humoser Sand; $1 \mathrm{U}=$ lehmiger Schluff; $\mathrm{N}=$ Nullerde) je Gefäß; Starkregenereignis zehn Wochen nach Gülleaufbringung

\subsubsection{Verlagerbarkeit im bewirtschafteten Großlysimeter}

\subsubsection{Tetrazyklin}

\section{Sickerwasser - Versuchsjahr 1}

Ingesamt fielen im Probennahmezeitraum 2001 unter Mais 299 und unter Roggen 216 Proben an. Die Analyse der Wasserproben erfolgte zunächst chronologisch. Bei allen Lysimetern erfolgte die Sickerwasseruntersuchung bis zur 37. KW. Die Wasserproben ausgewählter Lysimeter (Kriterien: positiver TC-Nachweis; TC-Gaben $>40 \mathrm{mg}$ je Lysimeter) wurden zusätzlich bis einschließlich der 50. KW analysiert. Dieses Verfahren hatte zur Folge, dass $82 \%$ der Wasserproben unter Mais und $67 \%$ der Wasserproben unter Roggen analysiert wurden.

In den analysierten Sickerwasserproben unter Roggen $(n=145)$ konnte kein Tetrazyklin oberhalb der Bestimmungsgrenze von $1 \mu \mathrm{g} \mathrm{l}^{-1}$ nachgewiesen werden. Dagegen wurde in 4 von 245 analysierten Sickerwässern unter Mais eine TC-Konzentration oberhalb $1 \mu \mathrm{g} \mathrm{1^{-1 }}$ in Größenordnungen von 1 bis $3 \mu \mathrm{g} \mathrm{l}^{-1}$ ermittelt. Diese vier Befunde traten ausschließlich in den ersten vier Wochen nach der Gülleapplikation auf (Tabelle 31). 
Tabelle 31: Tetrazyklin (TC)-Gehalte $\left(\mu \mathrm{g}^{-1}\right)$ im Sickerwasser unter Mais in den ersten vier Wochen nach Gülleausbringung, Durchgang 2001 ( 1 = humoser Sand I; $2=$ humoser Sand II)

\begin{tabular}{lcccccc}
\hline $\begin{array}{l}\text { Lysimeter- } \\
\text { Nr./ }\end{array}$ & Bodenart & $\begin{array}{c}\text { TC-Menge } \\
\left(\mathbf{m g ~ k g}^{-1}\right)\end{array}$ & $\begin{array}{c}\text { 1. Woche } \\
(\mathbf{1 9 .} \mathbf{K W})\end{array}$ & $\begin{array}{c}\text { 2. Woche } \\
(\mathbf{2 0 .} \mathbf{K W})\end{array}$ & $\begin{array}{c}\text { 3. Woche } \\
(\mathbf{2 1 .} \text { KW })\end{array}$ & $\begin{array}{c}\text { 4. Woche } \\
(\mathbf{2 2} \text {. KW) }\end{array}$ \\
\hline 1 & 1 & 0,0 & - & - & - & - \\
25 & 1 & 0,0 & - & - & - & - \\
\hline 2 & 1 & 22,5 & 1,3 & - & - & - \\
26 & 1 & 22,5 & - & - & - & - \\
\hline 3 & 1 & 0,0 & - & - & - & - \\
27 & 1 & 0,0 & - & - & - & - \\
\hline 4 & 1 & 45,0 & - & - & - & - \\
28 & 1 & 45,0 & - & - & - & - \\
\hline 13 & 1 & 67,2 & - & - & - & 3,1 \\
35 & 1 & 67,2 & - & - & - & - \\
\hline 49 & 1 & 67,3 & - & - & - & - \\
61 & 1 & 67,3 & - & - & - & - \\
\hline 58 & 2 & 134,6 & - & - & 0,9 & - \\
65 & 2 & 134,6 & - & - & 1,2 & - \\
\hline
\end{tabular}

kursiv: Wert unterhalb Bestimmungsgrenze von $1 \mu \mathrm{g} t^{1}$, aber durch Referenzlabor bestätigt - = kein Befund

Die Referenzmessung durch die Zentrumsabteilung für Lebensmitteltechnologie und toxikologie der Tierärztlichen Hochschule Hannover bestätigte die gemessenen Konzentrationen in vergleichbarer Größenordnung (siehe Anhang, Tabelle 43).

Wie aus den Abbildung 28 und Abbildung 29 hervorgeht, traten die positiven TC-Befunde jeweils in Wochen mit nur geringem Sickerwasseranfall auf. Lysimeter 2 und 13 waren mit humosem Sand (1) befüllt, Lysimeter 58 und 65 dagegen mit einem humosem Sand (2) anderer Herkunft.

\section{Sickerwasser - Versuchsjahr 2}

Im zweiten Versuchsjahr erfolgte die Probennahme aufgrund der Erfahrungen aus dem Vorjahr bis zur 40. KW (Maisernte). Auf diese Weise fielen unter Mais 65 und unter Roggen 91 Wasserproben an, die sämtlich auf Tetrazyklin untersucht wurden. In keinem Fall konnte Tetrazyklin im Sickerwasser in einer Konzentration oberhalb $1 \mu \mathrm{g} \mathrm{l^{-1 }}$ nachgewiesen werden. 


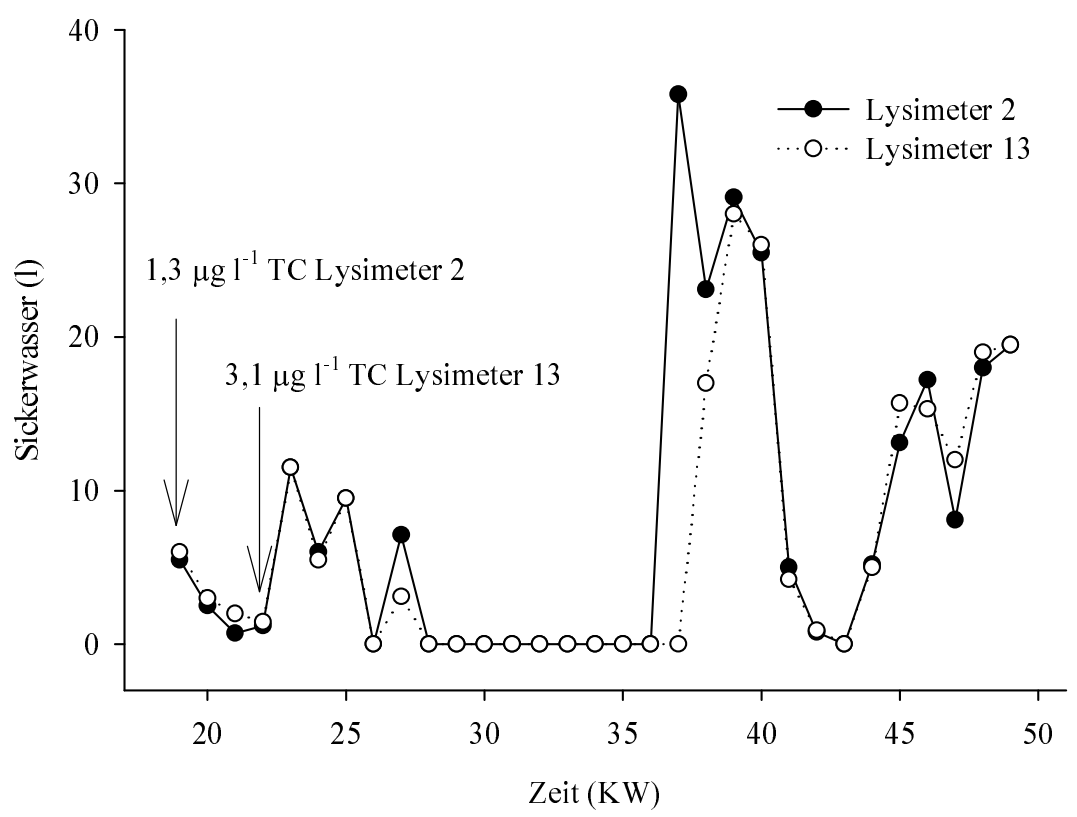

Abbildung 28: Sickerwasseranfall unter Lysimeter 2 und 13 (humoser Sand 1), Mais, Durchgang 2001, Tetrazyklin (TC)-Befund: 19. Kalenderwoche (Lysimeter 2) und 22. Kalenderwoche (Lysimeter 13)

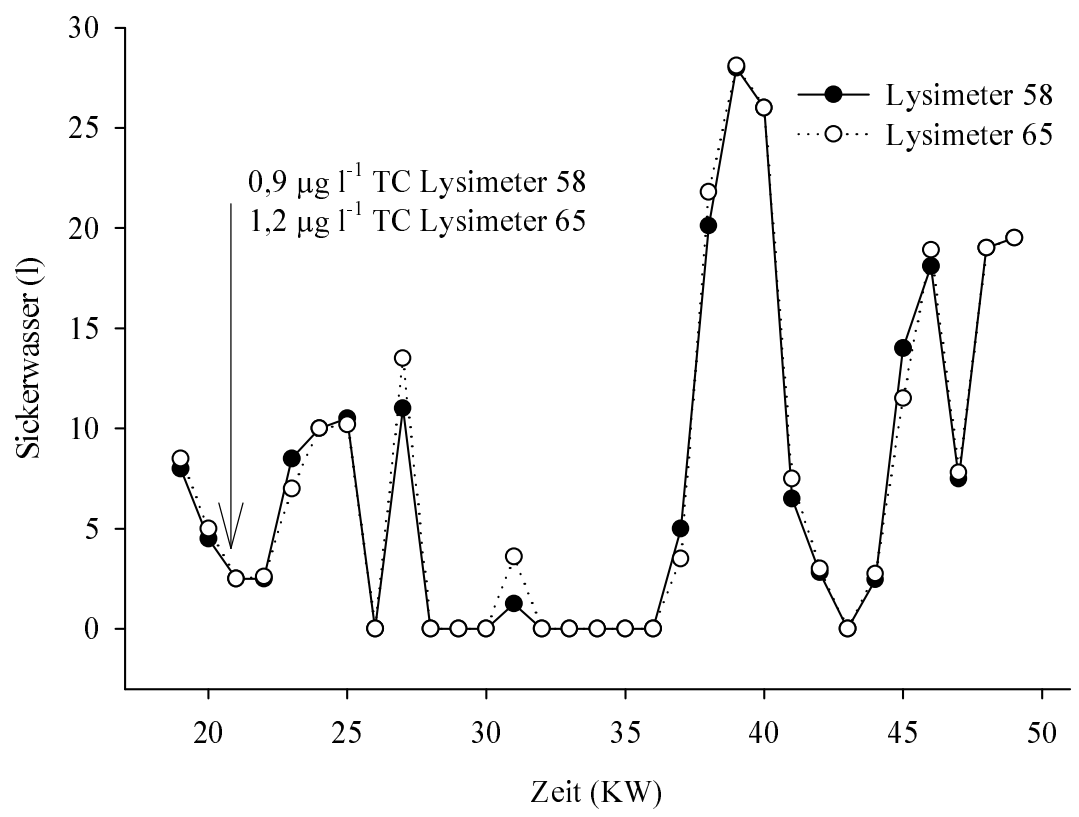

Abbildung 29: Sickerwasseranfall unter Lysimeter 58 und 65 (humoser Sand 2), Mais, Durchgang 2001, Tetrazyklin (TC)-Befund: 21. Kalenderwoche (Lysimeter 58 und Lysimeter 65) 


\section{Boden}

Bodenproben der Nullvarianten bestätigten, dass die Lysimeter vor der TC-Applikation kein Tetrazyklin enthielten. Dagegen konnte als Nebenbefund Chlortetrazyklin in unterschiedlichen Konzentrationen nachgewiesen werden, was auf die Verwendung CTChaltiger Gülle in den Vorjahren schließen lässt (siehe Anhang, Tabelle 46). Trotz der CTCKonzentration in Höhe von 2 bis maximal 25,9 $\mathrm{g} \mathrm{kg}^{-1}$ wurde jedoch in keinem Fall CTC im Sickerwasser gefunden. Im Folgenden sind die Bodenergebnisse ausgewählter Lysimeter in Abhängigkeit von der Fruchtart dargestellt (Abbildung 30).

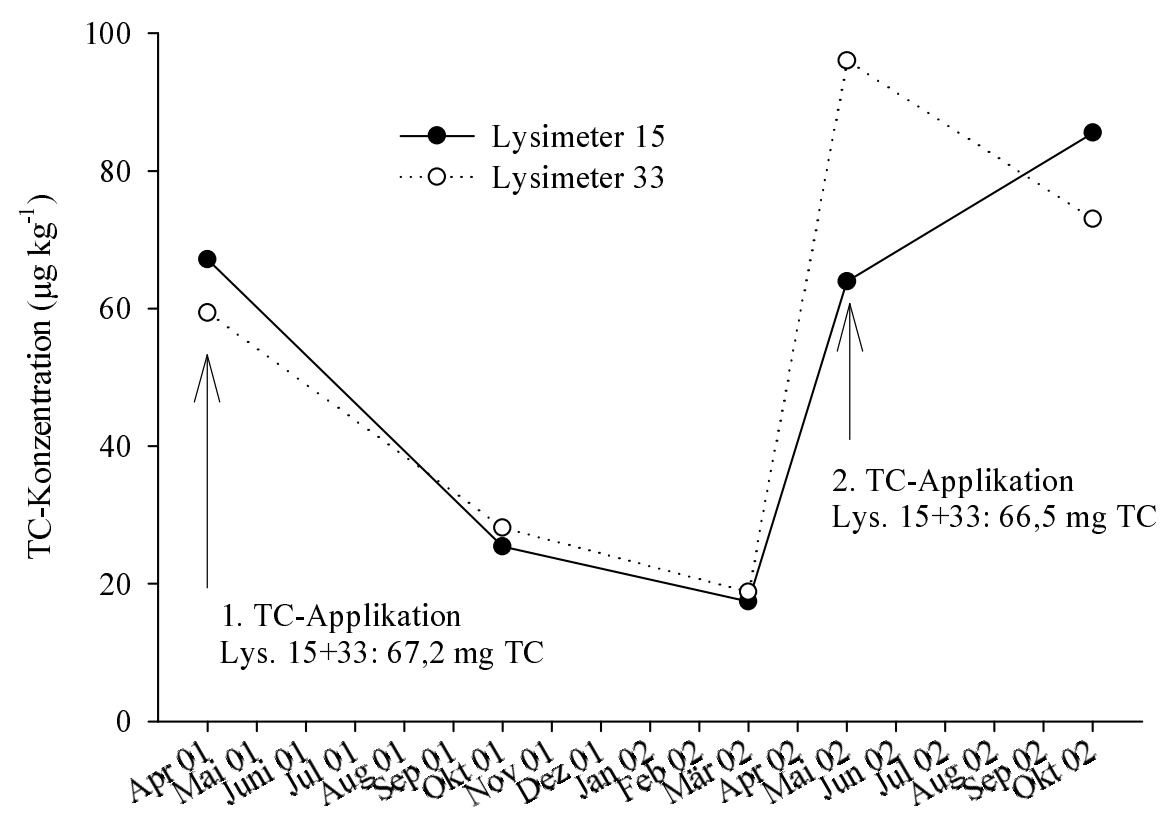

Abbildung 30: Verlauf der Tetrazyklin (TC)-Konzentration im Boden bei ausgewählten Lysimetern, Fruchtart Roggen, Zeitraum Frühjahr 2001 bis Herbst 2002

Die anfangs nachweislich unbelasteten Böden zeigten bei wiederholter Gülle und somit Wirkstoffgabe eine ansteigende Tendenz hinsichtlich der TC-Gehalte. Der Boden von Lysimeter 33 wies z.B. im Mai 2001 nach der ersten TC-Applikation $59 \mu \mathrm{g} \mathrm{kg}^{-1}$ auf; nach erneuter TC-Applikation in gleicher Höhe im darauf folgenden Mai 2002 konnten $96 \mu \mathrm{g} \mathrm{kg}^{-1}$ festgestellt werden. Im Zeitraum Frühjahr 2001 bis März 2002 wurde offenbar nicht die gesamte TC-Menge im Boden abgebaut, so dass mit erneuter Wirkstoffgabe im April 2002 die TC-Konzentration den Vorjahreswert überstieg. Bei den Lysimetern mit der Fruchtart Roggen änderte sich die TC-Konzentration im Boden ab der zweiten TC-Gabe bis zum Oktober 2002 nur unwesentlich. Dieser Effekt kann durch die Probennahme 
bedingt sein, allerdings zeigte er sich auch bei zwei Lysimetern mit Mais (Lysimeter 4 und 35) (Abbildung 31).

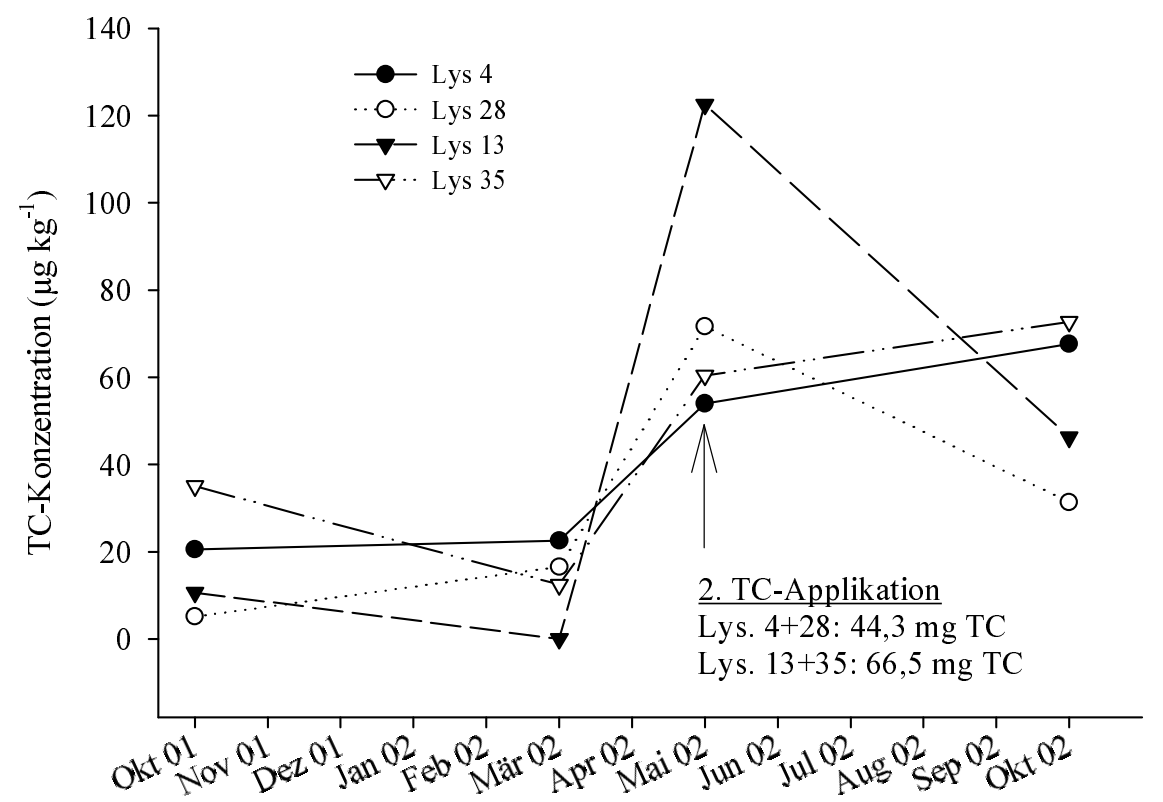

Abbildung 31: Verlauf der Tetrazyklin (TC)-Konzentration im Boden bei ausgewählten Lysimetern, Fruchtart Mais, Zeitraum Herbst 2001 bis Herbst 2002, 1. TCApplikation Mai 2001: Lys. 4 und 28: 45,0 mg; Lys. 13 und 35: 67,2 mg)

Die Boden-TC-Gehalte ausgewählter Lysimeter mit der Fruchtart Mais lagen im März, also kurz vor der zweiten Wirkstoffgabe, in vergleichbarer Höhe wie im Herbst 2001; deutlich abgebaut wurde das Tetrazyklin im Verlauf des Winters nicht. Am Beispiel des Lysimeters 13 soll der Jahresverlauf verdeutlicht werden: im Herbst 2001 wurde ein TCGehalt von $11 \mu \mathrm{g} \mathrm{kg}^{-1}$ nachgewiesen; mit erneuter Applikation von TC-dotierter Gülle stieg im April 2002 die TC-Konzentration auf $123 \mu \mathrm{g} \mathrm{kg}^{-1}$ an, um dann bis zum Herbst 2002 wieder auf ein allerdings gegenüber Herbst 2001 höheres Niveau von $46 \mu \mathrm{g} \mathrm{kg}^{-1}$ abzufallen.

Aus den vorliegenden Ergebnissen ist kein eindeutiger Einfluss der Dosierungsstufe abzuleiten. Lysimeter 13 enthielt zwar mit einem Messwert von $120 \mu \mathrm{g} \mathrm{kg}^{-1}$ TC im Frühjahr 2002 als höchstdosierte Variante die Maximalkonzentration unter den Lysimetern, demgegenüber wies jedoch Lysimeter 35 als dazugehörige Wiederholung mit gleicher Dosierung deutlich geringere TC-Konzentrationen auf. 


\subsubsection{Sulfadiazin}

\section{Sickerwasser}

Im Versuchsjahr 2002 wurde die Gülle neben Tetrazyklin auch mit Sulfadiazin dotiert. 65 Wasserproben unter Mais und 91 Wasserproben unter Roggen wurden im zweiten Versuchsjahr (2002) zusätzlich auf Sulfadiazin untersucht. Sulfadiazin konnte dabe oberhalb der Bestimmungsgrenze von $6 \mu \mathrm{g}{ }^{-1}$ nicht nachgewiesen werden.

\section{Boden}

Die Bodenproben aus den Nullvarianten wiesen ebenfalls kein Sulfadiazin auf, die Böden waren also nachweislich nicht mit diesem Wirkstoff belastet. Die Ergebnisse der Bodenuntersuchungen sind im Folgenden dargestellt (Abbildung 32).

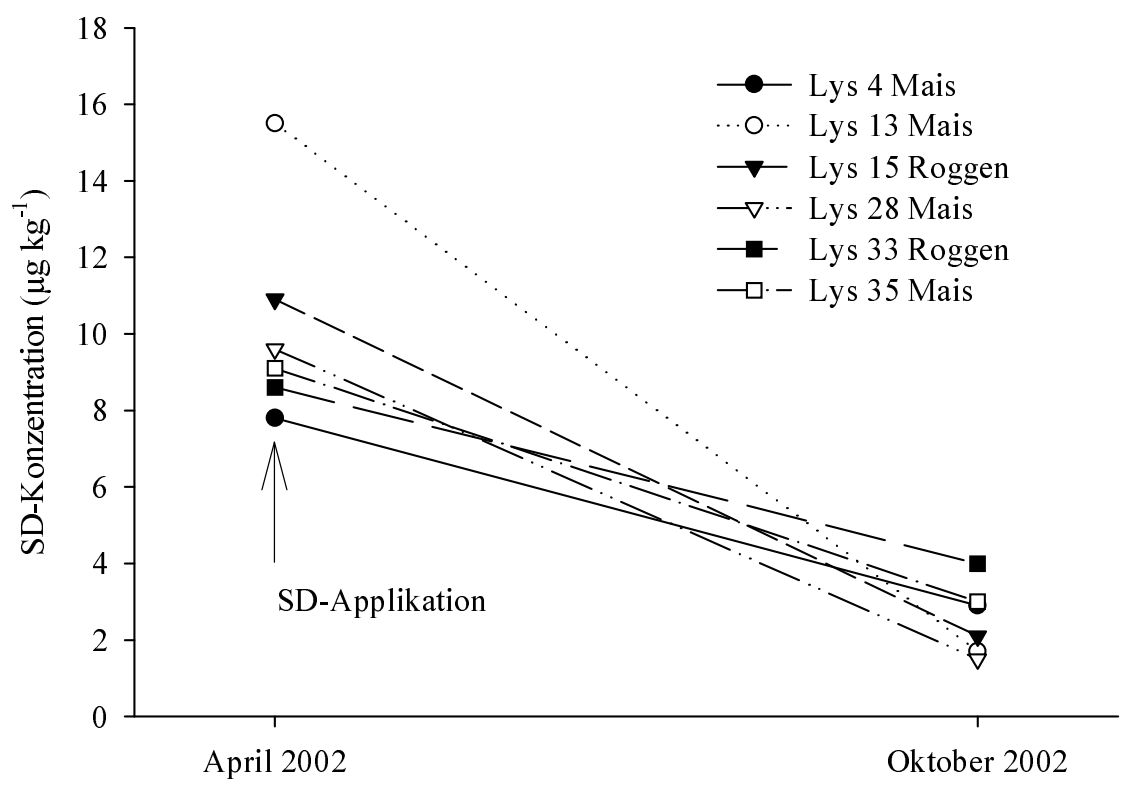

Abbildung 32: Verlauf der Sulfadiazin (SD)-Konzentration im Boden bei ausgewählten Lysimetern, Fruchtart Mais und Roggen, Zeitraum Frühjahr 2002 bis Herbst 2002 (Lys. 4 + 28: 44,3 mg SD; Lys. $13+35+15+33: 66,5$ mg $\mathrm{SD})$

Trotz gleicher Dosierung des Wirkstoffes erreichte Sulfadiazin im Gegensatz zu Tetrazyklin im Boden nur eine Maximalkonzentration von $16 \mu \mathrm{g} \mathrm{kg}^{-1}$ und lag damit deutlich unter den ermittelten TC-Gehalten. Weiterhin erfolgte eine deutliche Abnahme über den Probennahmezeitraum, so dass ein halbes Jahr später im Herbst 2002 nur noch Konzentrationen von maximal $4 \mu \mathrm{g} \mathrm{kg}^{-1}$ nachgewiesen werden konnten. 


\section{Diskussion}

Die in der vorliegenden Arbeit bearbeiteten Wirkstoffleitgruppen Tetrazykline und Sulfonamide werden in großem Umfang in der Nutztierhaltung als Breitband-Antibiotika eingesetzt. Über die in den tierischen Ausscheidungen anfallenden Mengen und deren Umweltverhalten nach Eintrag über Wirtschaftsdünger wie Gülle oder Festmist ist derzeit noch wenig bekannt. Diese Arbeit hatte daher zum Ziel, die Persistenz von Tetrazyklin in Masthähnchenfestmist sowie die Mobilität von Tetrazyklin und Sulfadiazin in unterschiedlichen Böden zu untersuchen, um einen Beitrag zur Abschätzung der Umweltgefährdung hinsichtlich Umweltverhalten und potenziellen Austrägen ins Grundwasser leisten zu können. Zusätzlich wurden Screening-Untersuchungen von anonymen Schweinegülleproben aus zwei Winterhalbjahren durchgeführt, um Anhaltspunkte über die tatsächliche Belastung der Wirtschaftsdünger in der Praxis zu erhalten.

\subsection{Modellversuche zur Persistenz von Tetrazyklin in Masthähnchenfestmist}

Im durchgeführten Versuchsansatz wurde angestrebt, die Persistenz von Tetrazyklin in praxisnahen Lagerungsvarianten $\mathrm{zu}$ testen, um eine Bewertungsgrundlage $\mathrm{zu}$ erhalten. Festmist aus der Geflügelmast wird häufig entweder direkt auf das Feld aufgebracht oder am Feldrand bzw. in befestigten Lagerstätten zwischengelagert. Dieser Mist wird in der Regel nicht umgesetzt, sondern über mehrere Wochen den natürlichen Witterungsbedingungen ausgesetzt und entspricht damit annähernd den Bedingungen für Stapelmist in den in dieser Arbeit durchgeführten Versuchen

Die natürlichen Witterungsbedingungen konnten im Versuchsmaßstab nicht betrachtet werden, da für eine Vergleichbarkeit der Ergebnisse konstante Umgebungsbedingungen zum Beispiel für Temperatur und Feuchte notwendig waren. Unter Praxisbedingungen würde aus arbeitswirtschaftlichen Gründen ein Umsetzen des Festmistes höchstens einmalig erfolgen. Daher wurde entschieden, bei Experiment 1 in einer Lagerungsvariante den Festmist einmal nach sechs Wochen zu durchmischen und neu aufzuschichten und bei Experiment 2 nach vier Wochen, jeweils also nach der Hälfte der Lagerungszeit. Das Ziel war es, je Experiment eine Variante als Rotte- und eine Variante als Stapelmist zu lagern. Es muss allerdings kritisch festgestellt werden, dass die gewählte Versuchsanstellung nur bedingt geeignet war, deutliche Unterschiede in den Lagerungsbedingungen (z. B. 
Belüftung, Rottetemperatur) zu generieren. Ein Vergleich der Lagerungssysteme Rottebzw. Stapelmist war daher nur ansatzweise möglich.

\section{Experiment 1}

Der Temperaturanstieg während der Kompostierung in der initialen Periode (Tag 0: $22^{\circ} \mathrm{C}$; Tag 6: $55^{\circ} \mathrm{C}$ ) ging mit der stärksten Konzentrationsabnahme einher (ca. 70 \% (Rottemist) bzw. $73 \%$ (Stapelmist) Rückgang der TC-Konzentration bei Dosierung 0,5 g TC kg-1 Futter, ca. 46 \% (Rottemist) bzw. 74 \% (Stapelmist) Rückgang bei Dosierung 1,0 g TC kg-1 Futter). Dieser Temperaturanstieg war bei Variante $1,0 \mathrm{~g} \mathrm{TC} \mathrm{kg}^{-1}$ Futter zu Beginn der Lagerung leicht verzögert; die höchste Rottetemperatur wurde in den Kontrollbehältern erreicht. Möglicherweise wirkte sich also der TC-Gehalt negativ auf die an der Kompostierung beteiligten Mikroorganismen aus, sodass eine Hemmung der Mikroflora den Rotteprozess verzögert anlaufen ließ.

Zur Wirkung von Antibiotika in Festmist sind bisher praktisch keine Literaturquellen verfügbar. Es wurden jedoch in begrenztem Umfang Studien zur Wirkung von Antibiotika in Biogasreaktoren durchgeführt. Sankvist et al. (1984) testeten die Wirkung einer Zugabe von $100 \mathrm{mg} \mathrm{l}^{-1}$ Oxytetrazyklin zu Schweinegülle an sechs aufeinander folgenden Tagen auf die Methanproduktion eines thermophilen Reaktors. Die Methanproduktion war nach Zugabe von Oxytetrazyklin signifikant um 50\% reduziert. Poels et al. (1984) dagegen untersuchten ebenfalls in Biogasreaktoren die potenziell hemmende Wirkung von Chlortetrazyklin, Tylosin, Erythromycin, Chloramphenicol, Bacitracin und Virginiamycin auf die Methanbakterien. Die Antibiotika wurden in praxisüblichen Dosierungen direkt in die Schweinegülle dosiert. Die Autoren konnten bei diesen Konzentrationen keine hemmenden Effekte auf die Methangasproduktion messen, in höher gewählten Konzentrationen allerdings wirkten sich Bacitracin und Virginiamycin negativ auf die Methanproduktion aus.

Rotte- und Stapelmist zeigten keinen signifikant unterschiedlichen Temperaturverlauf. Der Rottemist wies dagegen relativ zur jeweiligen Ausgangskonzentration geringere EndTCKonzentrationen auf als Stapelmist. Insgesamt waren aber während der Lagerungsdauer hohe Schwankungen in der TC-Konzentration zu verzeichnen, der Rückgang verlief sehr ungleichmäßig. Unklar ist, ob ein Konzentrationsrückgang tatsächlich auch einen Abbau des Tetrazyklins bedeutete, da auch eine vorübergehende Festlegung des Wirkstoffes an organische Bestandteile des Festmistes oder aber auch eine Komplexbildung mit zwei- und dreiwertigen Kationen in Frage kommt (siehe Kapitel 2.2.; Loke et al. (2002)). 
Am Ende der dreimonatigen Lagerdauer konnten im Mist der niedriger dotierten Variante $\left(0,5 \mathrm{~g} \mathrm{TC} \mathrm{kg}^{-1}\right.$ Futter) noch $3,5 \mathrm{mg} \mathrm{kg}^{-1} \mathrm{TC}$ (Rottemist) nachgewiesen werden $(13 \% \mathrm{der}$ Ausgangskonzentration), im Stapelmist dieser Variante war dagegen kein Tetrazyklin oberhalb der Bestimmungsgrenze von $1 \mathrm{mg} \mathrm{kg}^{-1}$ mehr detektierbar. Die höher dotierte Variante (1,0 $\mathrm{g} \mathrm{TC} \mathrm{kg}^{-1}$ Futter) enthielt als Rottemist noch 9,8 $\mathrm{mg} \mathrm{kg}^{-1}$ und als Stapelmist 22,0 $\mathrm{mg} \mathrm{kg}^{-1}$ Tetrazyklin (17,6 bzw. 22,0 \% der Ausgangskonzentration). Möglicherweise lag durch die höhere TC-Ausgangskonzentration der Variante $1,0 \mathrm{~g} \mathrm{TC} \mathrm{kg}^{-1}$ Futter $(55,7$ bzw. 100,1 mg TC kg ${ }^{-1}$ ) eine Hemmung der Mikroorganismen und somit des TC-Abbaus vor. Hinweise auf potenzielle Hemmwirkungen von Antibiotika finden sich in den Arbeiten von Sankvist et al. (1984) und Poels et al. (1984).

Die Ursachen für stärkere Konzentrationsschwankungen bei Rotte- als bei Stapelmist könnten in der Probennahme liegen. Durch die Inhomogenität des Festmistes wurden bei der Probennahme Kot und Stroh in unterschiedlichen Gewichtsanteilen entnommen, was $\mathrm{zu}$ einer Verzerrung der tatsächlich vorherrschenden TC-Konzentration führte. Die Korrektur der Ergebnisse auf den Kohlenstoffgehalt (C) der Proben bestätigte diese Annahme, da die Konzentrationsverläufe auf C korrigiert gleichmäßiger waren. Wie in Kapitel 2.2 beschrieben gehen Tetrazykline eine Vielzahl von chemischen Bindungen mit Matrixbestandteilen ein (Kroker et al. 1996; Kroker 2002); im Fall des Festmistes liegt eine verstärkte Bindungsneigung zu den Kot- statt zu den Stroh- bzw. Kohlenstoffanteilen vor.

Möglich wäre aber auch, dass die stärkeren Konzentrationsschwankungen im Rottemist wenigstens teilweise durch partielle Mobilisierung von schon festgelegtem Tetrazyklin durch Aufbrechen bestimmter Bindungsformen ihren Ursprung haben (Thiele-Bruhn 2003a). Grafe (2001) wies diesen Effekt bei Tetrazyklin in gelagerter Schweinegülle nach: zu Beginn der Lagerung stieg die TC-Konzentration in den Schweinegülleproben zum Teil über die Ausgangskonzentration hinaus an, um dann wieder abzufallen. Einen ähnlichen Effekt fanden Berger et al. (1986) bei Sulfadimidin in Schweinegülle. Sie führten dies auf mikrobiell-enzymatische Prozesse zurück.

Insgesamt lassen die Ergebnisse daher darauf schließen, dass eine repräsentative Probennahme wenigstens beim locker geschichteten Rottemist nicht in der Form möglich war wie beim komprimierten Stapelmist. Weiterhin sind die stark unterschiedlichen Ausgangskonzentrationen von Tetrazyklin im Masthähnchenfestmist zu diskutieren: eventuell ist trotz gleicher Verabreichungsmenge an Tetrazyklin je Masthähnchengruppe 
(also entweder 0,5 oder 1,0 $\mathrm{g} \mathrm{TC} \mathrm{kg}^{-1}$ Futter) während der Hähnchenmast unterschiedlich viel Tetrazyklin durch die Tiere aufgenommen worden (Entmischung von Futter und Wirkstoff während des Aufenthalts im Futterautomaten) und durch die Ausscheidung der Masthähnchen in den Mist gelangt. Möglich wäre aber auch, dass eine vollständig homogene Probe des jeweiligen Ausgangsmaterials nicht gewonnen werden konnte. Aus diesem Grund war es letztendlich nicht möglich, Durchschnittswerte für denTC-Abbau je Fütterungsvariante zu erhalten, vielmehr mussten die Komposter als Einzelwerte behandelt werden. Eine vorangehende Mast mit TC-Applikation an Masthähnchen ist im Gegensatz zu ähnlichen Versuchen in der Schweine- und Rinderhaltung (Elmund et al. 1971; Langhammer 1989; Grafe 2001) offensichtlich aufgrund der zwangsläufigen Inhomogenität des Festmistes als Kot-Stroh-Gemisch eine eher ungeeignete Methode, um eine homogene TC-Konzentration im Festmist für daran anschließende Kompostierungsversuche zu erhalten.

\section{Experiment 2}

Im Gegensatz zu Experiment 1 wurde der in Experiment 2 verwendete Festmist mit Tetrazyklin in einer Höhe von $45 \mathrm{mg}$ TC je kg Mist dotiert; es wurde also nur eine Konzentrationsstufe eingestellt. Während der zweimonatigen Lagerung erfolgte der stärkste TC-Abbau wieder in den ersten 14 Tagen nach Aufsetzen des Festmistes (Rottemist: ca. $78 \%$ Abbau; Stapelmist: ca. 90\%). In dieser Zeit stieg die Temperatur anfangs schnell von $22^{\circ} \mathrm{C}$ auf etwa $48^{\circ} \mathrm{C}$ an, um dann einige Tage konstant hoch zu bleiben. Im weiteren Verlauf der Lagerung wurden die TC-Konzentrationen beider Lagerungsvarianten weiter verringert; nach zwei Monaten enthielt der Rotemist noch durchschnittlich 4,9 $\mathrm{mg} \mathrm{kg}^{-1}$ (9,6\% der Ausgangskonzentration), der Stapelmist noch durchschnittlich 2,7 $\mathrm{mg} \mathrm{kg}^{-1}$ (6,2\% der Ausgangskonzentration).

In Experiment 2 war es aufgrund der einheitlicheren Ausgangskonzentrationen möglich, mittlere TC-Verläufe je Lagerungsvariante zu bestimmen. In Experiment 2 wies Stapelmist unerwarteter Weise am Ende der Lagerung geringere TC-Konzentrationen auf als Rottemist. Nach dem Umsetztermin des Rottemistes stieg die TC-Konzentration im Festmist dieser Variante wieder etwas an, wogegen der Verlauf im Stapelmist keinen Anstieg verzeichnete. Möglicherweise werden durch die vermehrte Sauerstoffzufuhr bedingt durch das Umsetzen mikrobielle Prozesse angeregt (Bilitewski et al. 1990; Bayerisches Landesamt für Umweltschutz 1999), die zu einer Remobilisierung von schon festgelegtem Tetrazyklin führen. Denkbar ist jedoch auch, dass durch das Durchmischen 
des Festmistes Material aus dem Randbereich des Komposters, in welchem wie bei Roth (1994) beschrieben geringere Temperaturen herrschen und so vermutlich ein verringerter TC-Abbau stattfand, in die Probennahmezone gelangt ist und $\mathrm{zu}$ höheren TCKonzentrationen führte.

Es traten ähnliche Schwankungen im Konzentrationsverlauf beider Lagerungsvarianten auf wie schon bei Experiment 1 beobachtet. Als Erklärungsansatz kommen wieder die Inhomogenität des Kot-Stroh-Gemisches sowie mögliche Remobilisierungseffekte des Tetrazyklins und seiner Abbauprodukte in Betracht.

Die Temperaturen in den unbelasteten Kontrollen lagen ähnlich wie bei Experiment 1 mit bis $\mathrm{zu} 3{ }^{\circ} \mathrm{C}$ deutlich über denen der TC-belasteten Varianten. Dies unterstreicht die Vermutung, dass die TC-Konzentrationen von 25 bis $100 \mathrm{mg} \mathrm{kg}^{-1}$ (Testbereich von Experiment 1 und 2) hoch genug sind, um bestimmte Mikroorganismen zu hemmen(siehe Poels et al. 1984; Sankvist et al. 1984). Am Rotteprozess sind vor allem aerobe und fakultativ aerobe Bakterien, Actinomyceten und Schimmelpilze beteiligt, deren Anteile sich je nach Rottephase temperaturabhängig verschieben (Bilitewski et al. 1990). Für zukünftige Arbeiten auf diesem Gebiet wäre es daher interessant zu ermitteln, aus welchen Einzelarten sich die Mikroorganismenpopulation im lagernden Festmist zusammensetzt und welche Mikroorganismen durch die Anwesenheit von Tetrazyklin gehemmt werden.

Hinweise auf derartige Effekte ergaben Studien, die sich wie Poels et al. (1984) und Sankvist et al. (1984) mit den Auswirkungen von Antibiotika in Biogasanlagen beschäftigten. So zeigten z.B. Massé et al. (2000), dass die Anwesenheit von Tetrazyklin und Penicillin in Schweinegülle die Methanproduktion im Biogasreaktor signifikant um 25 bis $35 \%$ verringerte. Sie vermuteten wie auch schon Fedler und Day (1985), dass durch die Anwesenheit dieser Antibiotika oder deren Metabolite die Methanbakterien in ihrer Aktivität gehemmt werden.

Hilpert et al. (1984) konnten in Methanfermentationsversuchen mit Klärschlamm bei einer Monensin-Natrium ${ }^{2}$-Konzentration von $1 \mathrm{mg} \mathrm{kg}^{-1}$ eine deutlich verminderte Biogasproduktion nachweisen. Ab Wirkstoffkonzentrationen von 10 bis $50 \mathrm{mg} \mathrm{kg}^{-1} \mathrm{kam}$ die Biogasproduktion zum Erliegen, was auf einen starken Rückgang der Anzahl methanogener Bakterien zurückgeführt wurde. In diesen Studien wurden jedoch nicht die Mechanismen untersucht bzw. diskutiert, die zu der möglichen Hemmung der

\footnotetext{
2 Anmerkung: Monensin-Natrium ist ein noch zugelassener pharmakologisch wirksamer Futterzusatzstoff.
} 
Methanbakterien führten, so dass daher in weiteren Versuchen die Persistenz von Tetrazyklin während einer vollständigen Kompostierung oder anaerober Vergärung unter gleichzeitiger Bestimmung der beteiligten Mikroorganismen und Beachtung der Qualitätsparameter für Kompost bzw. Biogas gezielt getestet werden sollte.

Auch die gezielte Beeinflussung der Umsetzungsvorgänge durch Zusätze wie Gesteinsmehl oder Biokompost könnte untersucht werden, da festgestellt werden musste, dass reiner Masthähnchenfestmist mit einem C/N-Verhältnis von maximal 10:1 für eine optimale Kompostierung, bei der das C/N-Verhältnis zwischen 20 bis 35:1 liegen sollte, nicht ideal zusammengesetzt ist (Bayerisches Landesamt für Umweltschutz 1999; Zaied 1999).

Während für Tetrazyklin in Schweinegülle Halbwertszeiten zwischen 45 und 105 Tagen ermittelt wurden (Winckler und Grafe 2000), zeigte Tetrazyklin im gelagerten Masthähnchenfestmist einen deutlich schnelleren Konzentrationsrückgang (bei Experiment 1 in drei Monaten ca. 78 bis $100 \%$ Abbau; bei Experiment 2 in zwei Monaten ca. 90 bis $94 \%$ Abbau).

Für die mittlere Anfangskonzentration an Tetrazyklin im Masthähnchenfestmist aus Experiment 2 von $51,1 \mathrm{mg} \mathrm{kg}^{-1}$ im Rottemist und 43,6 $\mathrm{mg} \mathrm{kg}^{-1}$ im Stapelmist wurden Halbwertszeiten von 2,9 Tagen für Rottemist und 2,3 Tagen für Stapelmist (beide als potenzielle Funktion) berechnet (Abbildung 33). Mit einer exponentiellen Funktion konnte aufgrund der hohen Schwankungen im Konzentrationsverlauf nur ein unbefriedigendes Bestimmtheitsmaß $r^{2}=0,58$ für Rotte- und $r^{2}=0,61$ für Stapelmist erreicht werden; insofern sind die ermittelten Halbwertszeiten lediglich als Orientierungswerte zu verstehen. 


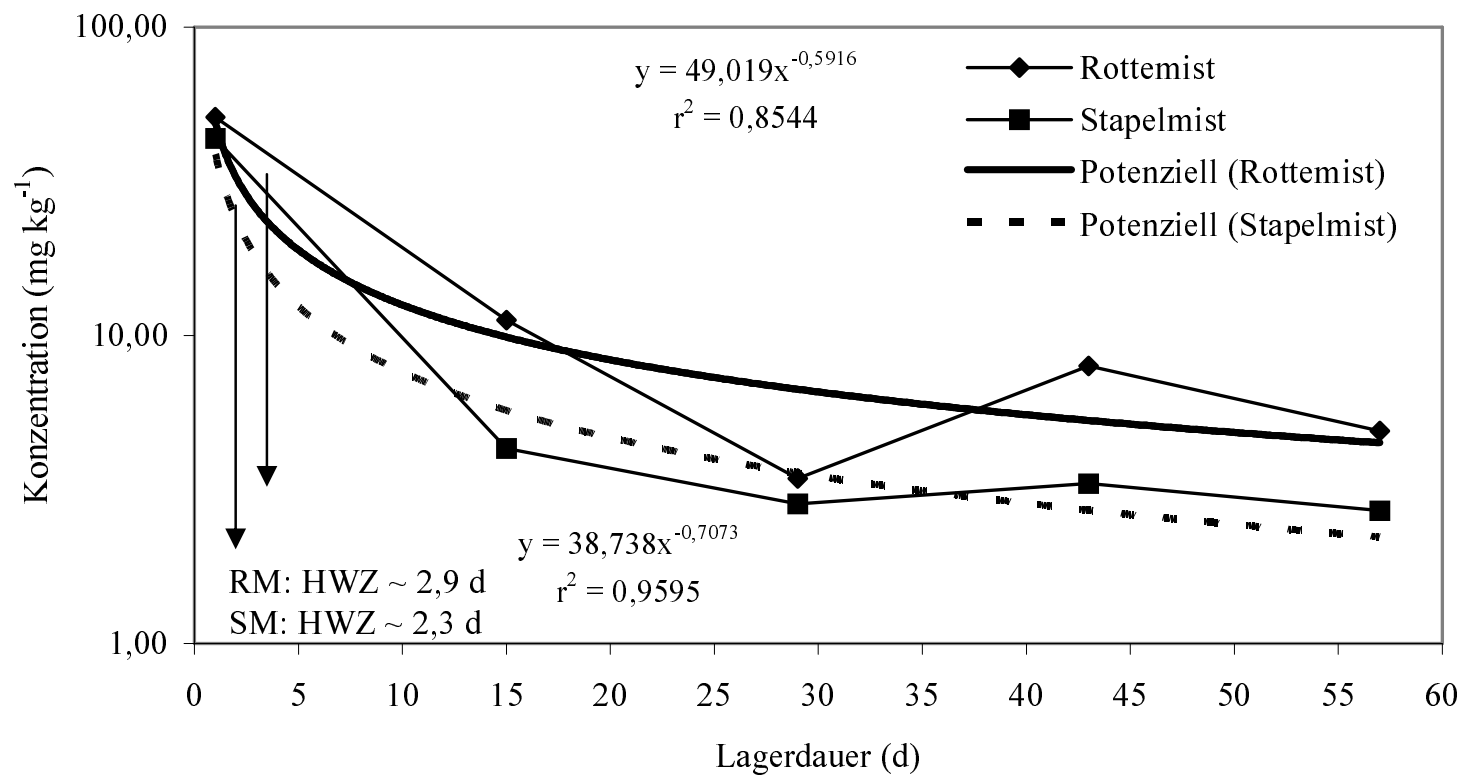

Abbildung 33: Halbwertszeiten (HWZ) von Tetrazyklin (TC) in Masthähnchenfestmist (Experiment 2) während der zweimonatigen Lagerung als Rotte (RM)bzw. Stapelmist (SM) in halblogarithmischer Darstellung (mittlere TC-Anfangskonzentration: Rottemist $=51,1 \mathrm{mg} \mathrm{kg}^{-1}$; Stapelmist $=43,6 \mathrm{mg} \mathrm{kg}^{-1}$ )

Hinsichtlich der Tetrazyklinpersistenz in Masthähnchenfestmist konnte zumindest ansatzweise eine Temperaturabhängigkeit beim Abbau des Antibiotikums gezeigt werden, wie es auch Gavalchin und Katz (1994) bei Chlortetrazyklin in Hühnerkot feststellten

\subsection{Nachweis ausgewählter Tetrazykline und Sulfonamide in Schweinegülle}

Insgesamt 168 Schweinegülleproben aus dem Jahr 1999/2000 von unterschiedlichen landwirtschaftlichen Schweineproduktionsbetrieben wurden auf den Gehalt an drei Tetrazyklinen (Tetrazyklin, Oxy- und Chlortetrazyklin) und zwei Sulfonamiden (Sulfadiazin, Sulfamethazin) untersucht. Grundsätzlich lag eine hohe Variabilität in den Wirkstoffgehalten vor. Die 1999/2000 am stärksten vertretenen Wirkstoffe waren Tetrazyklin und Sulfamethazin mit einem Anteil an den Gesamtproben von 38,7 bzw. 57,1 \%. Nur 35,1 \% der Proben enthielten keine der untersuchten Antibiotika.

Von den 176 anonymen Schweinegülleproben aus dem Screening 2001/2002 standen waren $12,5 \%$ der Proben bezüglich der fünf ausgewählten Antibiotika ohne Befund, d.h. es waren keine Wirkstoffkonzentrationen oberhalb der Bestimmungsgrenzen der in Kapitd 
3.1.2 beschriebenen Methode nachweisbar. Wieder stellten Tetrazyklin (49,4\%) und Sulfamethazin (49,3\%) die am stärksten vertretenen Antibiotika dar.

Zusätzlich konnte Sulfadiazin mit einem Anteil von 48,9 \% der Gesamtproben sehr viel häufiger nachgewiesen werden als im ersten, zwei Jahre zurückliegenden Screening, in dem Sulfadiazin nur einen Anteil von 8,3\% hatte. Hier fand offensichtlich zwischen den Probennahmezeiträumen 1999/2000 und 2001/2002 eine Änderung in der tierärztichen Verschreibungspraxis statt. Diese Verschiebung könnte durch die verstärkte Verwendung eines sulfadiazinhaltigen Präparates erklärt werden, welches im ersten Probennahmezeitraum nicht bzw. noch nicht sehr häufig eingesetzt wurde. Während diverse Präparate mit dem Wirkstoff Sulfamethazin auf dem deutschen Markt zugelassen sind, liegt nur ein Präparat mit dem Wirkstoff Sulfadiazin vor (Sulfadiazin+TMP C25/5 KS für Ferkel und Kälber; Lila Liste 2003). Dieses Präparat ist erst seit 1997 zugelassen und erhielt 2000 eine Zulassungsverlängerung (CHEVITA 2004). Somit könnte die möglicherweise gestiegene Verschreibungsrate dieses Präparates im Verlauf des Jahres 2000 zu dem Anstieg der Sulfadiazin-Anteile in der Gülle geführt haben.

Im Probensatz des Winterhalbjahres 1999/2000 enthielten 0,6 \% von 168 Proben alle fünf untersuchten Wirkstoffe, 2001/2002 waren es 1,1\% von 176 Proben. Am häufigsten waren die positiv befundenen Proben mit einem Wirkstoff belastet: 1999/2000 waren dies 26,8\% und 2002/2002 40,3\% der Proben.

Die Häufigkeit positiver Befunde entspricht insgesamt dem praxisüblichen, verbreiteten Einsatz dieser Wirkstoffe, der bereits in früheren Studien belegt worden war (Rassow und Schaper 1996; FEDESA 1999; Grafe 2001; Abbas et al. 2001; Thiele-Bruhn et al. 2003). Die ermittelten Sulfonamidkonzentrationen (2,9 bzw. $3,5 \mathrm{mg} \mathrm{kg}^{-1}$ Sulfadiazin; 4,3 bzw. $3,8 \mathrm{mg} \mathrm{kg}^{-1}$ Sulfamethazin) unterschritten trotz vergleichbarer empfohlener Dosierung deutlich die Werte für Tetrazykline (5,5 bzw. 7,3 $\mathrm{mg} \mathrm{kg}^{-1}$ Tetrazyklin); dies deutet auf eine geringere Persistenz der Sulfonamide in Gülle hin, was auch in den Arbeiten von Langhammer (1989) und Haller et al. (2002) vermutet wird.

Das große Spektrum der TS- und N-Gehalte (TS: 0,5 - 14,7 \% (1999/2000) bzw. 0,7 16,8 \% (2001/2002); N: 0,10 - 1,01\% (1999/2000) bzw. 0,15 - 1,05\% (2001/2002)) wies auf eine unterschiedliche Art der Probennahme hin. Wird die Schweinegülle vor der Probenentnahme aufgerührt, so vermischen sich Flüssig und Festphase. Bei unaufgerührter Gülle erfolgt die Beprobung häufig aus der oberen, weitgehend feststofffreien Phase. Durch die starken Adsorptionseigenschaften (Sithole und Guy 1987; 
Tolls 2001; Thiele-Bruhn 2003a) einiger Antibiotika (speziell Tetrazykline, siehe Kapitel 3.1.2) befindet sich generell in der dickflüssigen unteren Schicht mehr Wirkstoff als in der wässrigen Phase.

Obwohl sich prozentual mehr positive Proben in den Klassen ab 6\% TS-Gehalt in den Schweinegülleproben befanden, war für das Screening 1999/2000 für keine der untersuchten Substanzen ein signifikanter Unterschied zwischen den TSKlassen feststellbar. Im Screening 2001/2002 dagegen konnte für Tetrazyklin, Chlortetrazyklin und Sulfadiazin ein signifikanter Unterschied zwischen den TS-Klassen statistisch abgesichert werden. Vermutlich besteht also ein Zusammenhang zwischen dem TSGehalt der Proben und der Anzahl positiver Proben, wobei dieser substanzabhängig ist und in weiteren Versuchen überprüft werden müsste.

Für die Auswertung der Ergebnisse, deren Interpretation und Diskussion und für die Modellierung der Expositionen wurde jeweils der Median der positiv getesteten Proben herangezogen. Tabelle 32 zeigt die unterschiedlichen Ergebnisse der Medianwerte unter Berücksichtigung nur der positiven bzw. aller Proben je Screening. Für eine verlässliche Risikoabschätzung ist eine Berücksichtigung aller Proben einer Stichprobe, also auch der unbelasteten Proben, nicht sinnvoll, da sich so fast ausnahmslos Mediane von Null ergeben.

Tabelle 32: Gegenüberstellung der Medianwerte unter Berücksichtigung der positiv getesteten Proben bzw. aller Proben der beiden Schweinegülle-Probensätze

\begin{tabular}{|c|c|c|c|c|}
\hline \multirow[t]{2}{*}{ Substanz } & \multicolumn{2}{|c|}{ Screening 1999/2000 } & \multicolumn{2}{|c|}{ Screening 2001/2002 } \\
\hline & $\begin{array}{c}\text { Median } \\
\text { positiv. Proben } \\
\left(\mathrm{mg} \mathrm{kg}^{-1}\right)\end{array}$ & $\begin{array}{c}\text { Median aller } \\
\text { Proben } \\
\left(\mathrm{mg} \mathrm{kg}^{-1}\right)\end{array}$ & $\begin{array}{c}\text { Median } \\
\text { positiv. Proben } \\
\left(\mathrm{mg} \mathrm{kg}^{-1}\right)\end{array}$ & $\begin{array}{c}\text { Median aller } \\
\text { Proben } \\
\left(\mathrm{mg} \mathrm{kg}^{-1}\right)\end{array}$ \\
\hline Tetrazyklin & 5,5 & 0,0 & 7,3 & 0,0 \\
\hline Oxytetrazyklin & 4,8 & 0,0 & 5,1 & 0,0 \\
\hline Chlortetrazyklin & 4,4 & 0,0 & 5,6 & 0,0 \\
\hline Sulfadiazin & 2,9 & 0,0 & 3,4 & 0,0 \\
\hline Sulfamethazin & 4,3 & 2,0 & 3,8 & 0,0 \\
\hline
\end{tabular}

Eine Betrachtung des arithmetischen Mittels aller Proben kommt ebenfalls nicht in Frage, da dieses durch einige wenige Maximalwerte $\mathrm{zu}$ stark beeinflusst wird und damit $\mathrm{zu}$ überhöhten Expositionsabschätzungen führen würde. Der Median als Zentralwert einer 
Stichprobe ist in dieser Hinsicht ein sehr robustes Lagemaß (STAHEL 2000). Daher ist für verlässliche Expositionsabschätzungen der landwirtschaftliche Einzelbetrieb mit seiner spezifischen Schweinegülle entscheidend, denn im worst-case-Szenario würden Einzelflächen mit stark wirkstoffhaltiger Gülle beaufschlagt werden und dadurch könnten lokal mögliche Umweltwirkungen durch die ausgebrachten Wirkstoffe auftreten.

Abschließend soll eine Expositionsabschätzung in Anlehnung an Grafe (2001) in Bezug auf den Eintrag von Tetrazyklin in Boden unter Berücksichtigung der Belastung der in der vorliegenden Arbeit untersuchten Güllen versucht werden. Im Unterschied zu den worstcase-Bedingungen von $170 \mathrm{~kg}$ Stickstoff bei Grafe (2001) erfolgte die Berechnung unter der Annahme, dass etwa $100 \mathrm{~kg}$ Stickstoff je Hektar über Gülle ausgebracht wurden. Gemittelt über die Tetrazyklin-Belastung der Güllen (Median der positiven Proben) aus dem Winterhalbjahr 1999/2000 würden mit $100 \mathrm{~kg}$ N etwa $108 \mathrm{~g}$ Tetrazyklin auf den Hektar gelangen (Mittelwert: 177 g). 2001/2002 wären es sogar 128 g Tetrazyklin (Median der positiven Proben) (Mittelwert: 195 g). In Tabelle 33 sind die aus den Medianwerten der Wirkstoffkonzentration (Median der positiven Proben) abgeleiteten PEC/Boden-Werte aufgelistet:

Tabelle 33: PEC (Predicted Environmental Concentration) für ausgewählte Wirkstoffe in Boden auf Basis der Untersuchungsergebnisse aus Screening 1 und 2(Median der positiven Proben) bei verschiedenen Einarbeitungstiefen (ET)

\begin{tabular}{|c|c|c|c|c|c|c|c|c|c|c|}
\hline & \multicolumn{2}{|c|}{ TC } & \multicolumn{2}{|c|}{ OTC } & \multicolumn{2}{|c|}{ CTC } & \multicolumn{2}{|c|}{ SD } & \multicolumn{2}{|c|}{ SM } \\
\hline & 99/00 & $01 / 02$ & 99/00 & $01 / 02$ & 99/00 & $01 / 02$ & $99 / 00$ & $01 / 02$ & $99 / 00$ & $01 / 02$ \\
\hline $\begin{array}{l}\text { Gesamtdosis } \\
\left(\mathrm{g} \mathrm{ha}^{-1}\right)\end{array}$ & 108,4 & 127,9 & 134,4 & 76,6 & 91,5 & 99,0 & 72,9 & 79,4 & 91,7 & 74,3 \\
\hline \multicolumn{11}{|l|}{ PEC/Boden* } \\
\hline $\begin{array}{l}\text { ET: } 5 \mathrm{~cm} \\
\left(\mu \mathrm{gg}^{-1}\right)\end{array}$ & 144 & 170 & 179 & 102 & 122 & 132 & 97 & 106 & 122 & 99 \\
\hline$\left(\mu \mathrm{g} \mathrm{kg}^{-1}\right)^{* *}$ & 115 & 136 & 143 & 82 & 98 & 106 & 78 & 85 & 98 & 79 \\
\hline \multicolumn{11}{|l|}{ PEC/Boden* } \\
\hline $\begin{array}{l}\text { ET: } 10 \mathrm{~cm} \\
\left(\mu \mathrm{kg}^{-1}\right)\end{array}$ & 72 & 85 & 90 & 51 & 61 & 66 & 49 & 53 & 61 & 50 \\
\hline$\left(\mu \mathrm{gg}^{-1}\right)^{* *}$ & 57 & 68 & 72 & 41 & 49 & 53 & 39 & 42 & 49 & 40 \\
\hline $\begin{array}{lr}\text { TC: } & \text { Tetrazyklin } \\
* & : \text { Boden } \\
* * & : \text { Wirks } \\
& \text { Anteils }\end{array}$ & $\begin{array}{l}\text { OTC: } \mathrm{O} \\
\text { dichte: } 1 \\
\text { offkonze } \\
\text { (pauscha }\end{array}$ & $\begin{array}{l}\text { ytetrazyl } \\
00 \mathrm{~kg} / \mathrm{m} \\
\text { tration ir } \\
20 \% \text { an }\end{array}$ & $\begin{array}{l}\text { din; CTC } \\
\text { Boden } 1 \\
\text { genomme }\end{array}$ & $\begin{array}{l}\text { Chlorte } \\
\text { ch Abzl }\end{array}$ & azyklin; & $\begin{array}{l}\text { SD: Sulf } \\
\text { mikrobie }\end{array}$ & $\begin{array}{l}\text { diazin; } S \\
1 \text { unwirk }\end{array}$ & $\begin{array}{l}\text { M: Sulfan } \\
\text { amen Me }\end{array}$ & $\begin{array}{l}\text { nethazin } \\
\text { tabolit/E }\end{array}$ & imer- \\
\hline
\end{tabular}


Für Tetrazyklin ergaben sich PEC/Boden-Werte von 144 bzw. $170 \mu \mathrm{g} \mathrm{kg}^{-1}$ bei einer Einarbeitungstiefe der Gülle in den Boden von $5 \mathrm{~cm}$. Bei einer Einarbeitungstiefe von $10 \mathrm{~cm}$ betragen die berechneten PEC/Boden-Werte noch 72 bzw. $85 \mu \mathrm{g} \mathrm{kg}^{-1}$. Vergleichbare Werte wiesen Höper et al. (2002) für Tetrazyklin in Oberböden bis $30 \mathrm{~cm}$ Tiefe nach $\left(<50\right.$ bis $\left.158 \mu \mathrm{g} \mathrm{kg}^{-1}\right)$. Unter der pauschalen Berücksichtigung des antimikrobiell unwirksamen Epimers bzw. Metaboliten verringern sich die PEC/BodenWerte um $20 \%$. Für die Zulassung von Tierarzneimitteln schreibt die EMEA/CVMP/055/96-Leitlinie seit 1998 eine weiterführende ökotoxikologische Prüfung (Phase II) vor, wenn die PEC/Boden-Werte $100 \mu \mathrm{g} \mathrm{kg}^{-1}$ überschreiten. Aufgrund der Untersuchungsergebnisse der beiden Güllejahrgänge 1999/2000 und 2001/2002 kann davon ausgegangen werden, dass dieser Triggerwert wenigstens für Tetrazyklin, aber auch für Oxytetrazyklin oder Chlortetrazyklin bei entsprechender Gülleaufbringung erreicht werden kann.

Legt man die jeweiligen Maximalbelastungen der Gülle mit Tetrazyklin zugrunde (1999/2000: 44,4 mg TC kg-1 Gülle; 2001/2002: 45,7 mg TC kg-1 Gülle), würden im worstcase-Szenario 0,73 bis 1,23 kg Tetrazyklin je Hektar bei jeder Gülledüngung ausgebracht werden (PEC/Boden-Wert: $>800 \mu \mathrm{g} \mathrm{kg}^{-1}$ ). Dies stellt eine erhebliche Eintragsmenge dar und ist besonders vor dem Hintergrund problematisch, dass Tetrazyklin, aber auch Oxytetrazyklin als sehr persistent eingeschätzt werden und zumindest Tetrazyklin vermutlich im Boden akkumuliert (siehe Kapitel 2.4, Samuelsen et al. 1994; Rabølle und Spliid 2000; Höper et al. 2002).

Insgesamt bleibt festzustellen, dass entgegen der Absichtserklärungen bzw. Forderungen u.a. seitens der Arbeitsgemeinschaft Deutscher Tierzüchter (ADT 2001), den Tierarzneimitteleinsatz zu verringern und rationaler $\mathrm{zu}$ gestalten, die Ergebnisse dieses Screenings keine Reduzierung des Antibiotikaeinsatzes erkennen lassen.

\subsection{Modellversuche zur Mobilität von Tetrazyklin und Sulfadiazin in Boden}

Die Mobilität von Tetrazyklin und Sulfadiazin wurde in zwei unterschiedlichen Versuchsansätzen überprüft. Im Kick-Brauckmann-Gefäßversuch wurde die Fragestellung mit drei verschiedenen Böden, die jeweils als typisch für die Region WeserEms zu betrachten sind, im kleinen Maßstab bearbeitet. Mit dem Lysimeterversuch konnte im größeren Maßstab und praxisnäher gearbeitet werden. 


\section{Verlagerbarkeit im Kick-Brauckmann-Gefäß}

Im Sickerwasser aus humosem Sandboden und lehmigem Schluff konnten bei Starkregensimulation 48 Stunden nach Aufbringung tierarzneimittelhaltiger Gülle weder Tetrazyklin noch Sulfadiazin oberhalb der Bestimmungsgrenze von $2 \mu \mathrm{g} \mathrm{l^{-1 }}$ bzw. $6 \mu \mathrm{g} \mathrm{l^{-1 }}$ nachgewiesen werden. Dagegen lag bei zwei von drei Gefäßen mit Nullerde (auf Torfbasis) in allen Sickerwasserfraktionen Tetrazyklin in Spuren vor $\left(1,6-8,2 \mu \mathrm{g}^{-1}\right)$. Warum TC nur im Sickerwasser von zwei Gefäßen gefunden werden konnte, kann derzeit nicht erklärt werden. Bei Beregnung nach zehn Wochen konnten dagegen unabhängig von der Bodenart weder Tetrazyklin noch Sulfadiazin im Sickerwasser nachgewiesen werden.

Ursache für das Auftreten von TC im Sickerwasser unter Torfgemisch ist vermutlich der hohe Torfanteil der Nullerde. Dieser bedingt aufgrund seiner groben Struktur einen erhöhten Grobporenanteil im Boden. Derartige Grobporen ermöglichten unter Umständen eine Verlagerung nicht nur von Wasser sondem auch von Güllebestandteilen, an denen Tetrazyklin anhängig war. Dieser Kolloidtransport ist aus der Pestizidverlagerung bekannt (Hesketh et al. 2001; Strebe und Talbert 2001; McGechan und Lewis 2002). Auch für den Wirkstoff Avermectin wurde die Verlagerung durch Grobporen bzw. Hohlräume beschrieben (Gruber et al. 1990). Während Avermectin in kompakten Bodensäulen nur geringfügig verlagert wurde, nahm die Verlagerung durch präferentiellen Transport in den Schrumpfrissen eines schluffigen Lehms wesentlich đl.

Sithole und Guy (1987) wiesen nach, dass die Bindung von Antibiotika zu gelöstem organischen Material stärker ist als $\mathrm{zu}$ festen Bodenbestandteilen. Begleitende Untersuchungen des Ammonium-N- oder Phosphor-Gehalts zur Abschätzung eines möglichen Grobporenflusses in den Kick-Brauckmann-Gefäßen konnten aufgrund der geringen Probenvolumina allerdings nicht durchgeführt werden.

Einen weiteren Erklärungsansatz liefert der erhöhte Humus und fehlende Tonanteil in der Nullerde. Tetrazyklin lagert sich unterschiedlich stark an Tonmineralien und Humusbestandteile an (Tolls 2001; Rabølle und Spliid 2000; Sithole und Guy 1987). Nullerde hat eine vergleichsweise hohe Kationenaustauschkapazität (KAK), die offensichtlich durch den hohen Humus- und Strukturanteil hervorgerufen wird. Möglicherweise sind die Bindungskräfte zwischen Tetrazyklin und den Humusbestandteilen weniger stabil als die Bindungen, die mit Tonmineralien eingegangen werden. Dies könnte erklären, warum sich im humosen Sand und lehmigem Schluff trotz 
deutlich geringerer KAK kein Tetrazyklin verlagert hat, im Torfgemisch trotz hoher KAK jedoch TC ausgewaschen wurde.

Weiterhin ist ein Zusammenhang zwischen dem Kalziumgehalt des Bodens und der TCVerlagerung denkbar. Das Torfgemisch hatte einen etwa zehnfach höheren Kalziumgehalt als die anderen verwendeten Böden. Tetrazyklin bildet Chelatkomplexe mit Kalzium (Kroker 1996) und könnte so bei der Auswaschung von Kalzium mit dem Sickerwasser (Scheffer und Schachtschabel 1992) durch den Boden transportiert werden. Ausgewählte Sickerwasserproben der Böden wurden daher auf ihre Kalzium-Gehalte überprüft. Eine verstärkte Auswaschung von Kalzium beim Torfgemisch konnte nicht nachgewiesen werden. Dennoch könnten auch andere Kationen wie Magnesium oder Eisen als Transporter gedient haben. Limitierend für weitere Untersuchungen dieser Art war die geringe Probenmenge.

Nach dem frühen Starkregenereignis $(48 \mathrm{~h})$ lagen generell im Oberboden höhere Wirkstoffmengen vor als im Unterboden. Die Gehalte schwankten jedoch sehr stak zwischen den Bodenarten und waren offensichtlich vom Tongehalt des Bodens abhängig (lehmiger Schluff $(6,6 \%)>$ humoser Sand $(1,3 \%)>$ Nullerde (k.A.)). Ein derartiger Zusammenhang wurde auch von Thiele et al. (2002) am Beispiel des Sulfonamids Sulfapyridin beschrieben: bei Auftrennung einer Schwarzerde in organo-mineralische Partikelgrößen war die Adsorption von Sulfapyridin an die stabilen mineralorganischen Komplexe der Tonfraktion fast doppelt so hoch wie an die Sandfraktion mit ihrer vor allem partikulären organischen Substanz.

Grundsätzlich lagen die Sulfadiazin-Konzentrationen im Gefäßversuch 48 Stunden nach Gülleaufbringung bei gleicher Applikationsmenge um ein Vielfaches unter denen des Tetrazyklins. Einen prinzipiell schnelleren Abbau von Sulfonamiden hatte Langhammer (1989) bereits anhand von Sulfamethazin in Gülle nachgewiesen

Zehn Wochen später waren für beide Wirkstoffe deutlich geringere Konzentrationen im Boden nachweisbar. Der Rückgang der Gehalte im Vergleich zum Starkregenereignis nach 48 Stunden war bei Tetrazyklin stärker als bei Sulfadiazin, z.B. konnten im humosen Sand mit $553 \mu \mathrm{g} \mathrm{kg}^{-1} \mathrm{TC}$ nur noch $20 \%$ der früher ermittelten Konzentration nachgewiesen werden. Nach wie vor war aber für Tetrazyklin im Oberboden eine deutlich höhere Wirkstoffmenge zu finden als im Unterboden. Dies deutet einerseits auf einen Abbau und andererseits auf eine geringe Verlagerungsneigung des Wirkstoffes hin. Die starken Konzentrationsschwankungen, besonders im Oberboden bei Tetrazyklin (von 17,0 bis zu 
$339,7 \mu \mathrm{g} \mathrm{kg}^{-1}$ im lehmigen Schluff), können möglicherweise mit Gülleresten in der Bodenprobe in Zusammenhang gebracht werden.

Insgesamt belegen die Ergebnisse, dass Sulfadiazin im Boden sehr viel weniger stabil ist als Tetrazyklin. Für die stark festgelegten Tetrazykline wird ein nahezu ausschließlicher Abbau durch abiotische Prozesse angenommen (Halling-Sørensen 2000), wobei aber z.B. Photodegradation keine signifikante Bedeutung hat (Thiele-Bruhn et al. 2003). Andere Antibiotika wie z.B. die Sulfonamide werden vermehrt durch mikrobielle Degradationsprozesse wie oxidative Decarboxylierung und Hydroxylierung transformiert (McGrath et al. 1998; Al-Ahmad et al. 1999). Neben den physikochemischen Eigenschaften der Antibiotika beeinflussen Temperatur und die Austauscheigenschaften der Böden das Ausmaß und die Geschwindigkeit des Abbaus wesentlich(Gavalchin und Katz 1994). Trotz potenzieller Mobilität aufgrund geringer Sorptionsneigung an feste Bodenbestandteile (Tolls 2001) unterliegt Sulfadiazin daher eventuell stärkeren Abbauvorgängen im Vergleich zu Tetrazyklin (Boxall et al. 2002). Langhammer (1989) hatte bereits den raschen Abbau eines Sulfonamids in Gülle nachgewiesen Christian et al. (2003) dagegen wiesen in ihren Untersuchungen auf die hohe Stabilität von Sulfamethazin als Vertreter der Sulfonamide in Boden und Gülle hin, was im Gegensatz zu den hier ermittelten Ergebnissen steht.

Tetrazyklin zeigte zumindest in sandigen und lehmigen Böden eine ausgeprägte Stabilität im Boden sowie eine geringe Verlagerungsneigung. Damit werden die Untersuchungen von Rabølle und Spliid (2000), Höper et al. (2002) sowie Hamscher et al. (2002) bestätigt. Eine höhere Verlagerungsneigung in strukturreichen Moorböden, eventuell gebunden an Güllebestandteile, kann dagegen nicht ausgeschlossen werden.

\section{Verlagerbarkeit im bewirtschafteten Großlysimeter}

Im ersten Untersuchungsjahr wurde in vier Sickerwasserproben unter Mais - aber nicht unter Roggen - Tetrazyklin in einer Konzentration von mehr als $1 \mu \mathrm{g}^{-1}$ nachgewiesen. Dieser Befund ließ sich im zweiten Durchgang 2002 jedoch nicht reproduzieren. Das im zweiten Versuchsjahr zusätzlich eingesetzte Sulfadiazin war weder unter Mais noch unter Roggen nachweisbar. Damit liegen zumindest für das Lysimetermodell erstmals Hinweise auf eine Verlagerbarkeit von Tetrazyklin vor. Bisher wurden Tetrazykline nur in Oberflächenwasser (Hartig et al. 1999; Lindsey et al. 2001, Zhu et al. 2001) nachgewiesen, nicht aber in Sicker- bzw. Grundwasser. Lediglich in unmittelbarer Nähe zu so genannten 
Güllelagunen konnten antibiotische Wirkstoffe auch im Grundwasser nachgewiesen werden (Campagnolo et al. 2002).

Die betroffenen Lysimeter waren jeweils mit humosem Sand unterschiedlicher Herkunft befüllt. Es handelte sich demnach um keine gewachsenen Bodensäulen sondem um ein anthropogen beeinflusstes System.

Die positiven Tetrazyklin-Befunde unter Mais traten in den ersten vier Wochen nach Gülleapplikation bei relativ geringem Sickerwasserfluss auf. Anhand von Analysen durch ein Referenzlabor (Bestimmungsgrenze $0,1 \mu \mathrm{gl}^{-1}$ ) konnte jedoch weitgehend ausgeschlossen werden, dass zu Probennahmezeitpunkten mit höherem Sickerwasseranfall eine mit der verwendeten Nachweismethode nicht detektierbare TC-Auswaschung (Verdünnungseffekt) stattfand. Während unter Roggen, bei dem die Gülle nur oberflächlich aufgebracht wurde, kein Tetrazyklin im Sickerwasser nachgewiesen werden konnte, war dies unter Mais, bei dem die Gülle etwa $4 \mathrm{~cm}$ tief in den Boden eingearbeitet wurde, der Fall.

Kern et al. (2003) wiesen z.B. in Abwasserverregnungsversuchen nach, dass Bodenbearbeitung mit Pflug und Egge den präferentiellen Stofftransport zwar nicht erhöhte, aber dazu führte, dass ein größerer Teil der Beregnungslösung (Tracergemisch aus Bromid und dem Farbstoff Brilliant Blue FCF) in die obersten Zentimeter der Bodenmatrix eindrang und verblieb. Es ist jedoch gleichzeitig denkbar, dass die betroffenen Lysimeter mehr Grobporen (Wurzelbahnen, Wurmgänge etc.) enthielten und dies die Verlagerung von Gülle- oder Bodenpartikeln, an denen Tetrazyklin haftet, begünstigte. Laut Scheffer und Schachtschabel (1992) ist der Anteil der Grobporen mit etwa 30\% bei Sand am größten und sinkt mit dem Tongehalt und einem zunehmenden Gehalt an organischer Substanz der Böden, wobei letzteres zu einer Verschiebung von Grobporen zu mehr Mittel- und Feinporen führt. Ein Makroporenfluss wurde auch für den Nachweis von $E$. coli in unteren Bodenhorizonten und im Sickerwasser unter güllegedüngten Böden (Rahe et al. 1978) oder die Verlagerung von Pestiziden und Herbiziden (McGechan und Lewis 2002; Strebe und Talbert 2001), zum Teil durch Kolloidtransport (Hesketh et al. 2001), angenommen.

Die Schlussfolgerung, dass die Einarbeitung von Gülle in den Boden das Auswaschen von Tetrazyklin tatsächlich begünstigt, kann daher zu diesem Zeitpunkt nicht gezogen werden. Dagegen war keine Dosisabhängigkeit für das Auftreten oder die Höhe der Auswaschung ableitbar (Dosis der „positiven“ Lysimeter: 22,5 bis 134,6 mg TC). 
Im Hinblick auf eine realistische Risikobewertung muss berücksichtigt werden, dass es sich im vorliegenden Vorhaben um anthropogen beeinflusste Modelle handelt. Auch in so großen Bodenkörpern wie Lysimetern können die Bodenverhältnisse im Feld nur unvollständig simuliert werden. Die Versuche zur Mobilität von Tetrazyklin und Sulfadiazin in verschiedenen Böden haben daher lediglich Modellcharakter. Bei Gefäßversuchen besteht die Problematik, dass gestörte Böden eingesetzt werden, die von der Struktur her nicht direkt mit gewachsenen Böden vergleichbar sind.

Weiterhin handelte es sich bei den detektierten TC-Gehalten im Sickerwasser um sehr geringe Konzentrationen. So machte beispielsweise bei Lysimeter 13 der Anteil des detektierten Tetrazyklins $\left(3,1 \mu \mathrm{g} \mathrm{l}^{-1}\right.$ bei 1,451 Sickerwasseranfall $)$ an der Aufbringungsmenge (67,2 mg TC) lediglich 0,06\%o aus. Darüber hinaus war unter gewachsenen Böden auch mit hochempfindlichen Nachweisverfahren bisher kein Nachweis von Tetrazyklinen in mittels Saugkerzen gewonnenem Bodenwasser oder in oberflächennahem Grundwasser möglich (Höper et al. 2002). Einzige Ausnahme stellt die Arbeit von Campagnolo et al. (2002) dar, die in unmittelbarer Nähe zu Güllelagunen antibiotische Wirkstoffe im Grundwasser nachwiesen. Das Auswaschungsrisiko unter Praxisbedingungen ist daher als sehr gering einzuschätzen.

Die wiederholte Untersuchung des Lysimeterbodens auf Tetrazyklin ergab über die beiden Untersuchungsjahre einen Anstieg des Wirkstoffgehaltes im Boden. Im Herbst 2002 lag die TC-Konzentration nach erneuter Güllegabe im Frühjahr 2002 deutlich höher (bis $\left.80 \mu \mathrm{g} \mathrm{kg}^{-1}\right)$ als noch im Herbst $2001\left(<40 \mu \mathrm{g} \mathrm{kg}^{-1}\right)$. Es erfolgte demnach nur ein unvollständiger Abbau; dieser Effekt war unabhängig von Boden- oder Fruchtart. Dieses Ergebnis steht im Einklang mit der Studie von Höper et al. (2002). Ein Erklärungsansatz könnte der Umstand sein, dass das Jahr 2002 im Beprobungszeitraum trockener war als das Vorjahr. Von der 13. bis zur 40. KW 2002 (Beprobungszeitraum Winterroggen) fiel ca. $12 \%$, von der 19. KW bis zur 40. KW (Beprobungszeitraum Mais) sogar ca. 20\% weniger Niederschlag als im Vorjahr. Möglicherweise sind in einem insgesamt feuchteren Boden die Bedingungen zum Abbau des Tetrazyklins besser gegeben als in einem etwas trockeneren Milieu.

Sulfadiazin erreichte nach Applikation über Gülle wesentlich geringere Konzentrationen im Boden als Tetrazyklin und wurde über den Probennahmezeitraum deutlich abgebaut. Dies bestätigt die Befunde aus dem Gefäßversuch, wo Sulfadiazin ebenfalls in deutlich 
geringeren Konzentrationen nachweisbar war als Tetrazyklin. Sulfadiazin hat demnach eine geringere Persistenz in der Umwelt als Tetrazyklin.

Sowohl bei den Boden- (TC, SD) als auch den Sickerwasseruntersuchungen (TC) wurden zum Teil deutlich die sog. Triggerwerte der EMEA-Guideline für die Umweltbewertung von Tierarzneimitteln für Boden $\left(100 \mu \mathrm{g} \mathrm{kg}^{-1}\right)$ bzw. Wasser $\left(1 \mu \mathrm{g} \mathrm{l}^{-1}\right)$ überschritten, die weiterführende ökotoxikologische Untersuchungen (Phase II) einleiten. Hierbei ist allerdings $\mathrm{zu}$ berücksichtigen, dass sich diese Triggerwerte auf vorhergesagte Umweltkonzentrationen (Predicted Environmental Concentrations, PEC) auf Basis von worst-case-Szenarien beziehen. Bei den vorliegenden Untersuchungen wurden jedoch aus wissenschaftlichen Gründen teilweise deutlich über den in der landwirtschaftlichen Praxis zu erwartenden Dosierungen eingesetzt. Daher ist keine direkte Übertragbarkeit der in diesem Forschungsvorhaben ermittelten Gehalte gegeben. Auf Grundlage von Praxisdaten und experimentell erarbeiteten Halbwertszeiten für TC in Schweinegülle hatten jedoch bereits Winckler und Grafe (2000) Triggerwertüberschreitungen für die PEC-Boden bei TC-Anwendung in der Schweinemast ermittelt.

Obwohl mit der vorliegenden Arbeit ein Beitrag zur Abschätzung der Umweltgefährdung einzelner Tetrazykline und Sulfonamide versucht wurde, ergibt sich auf Basis der Ergebnisse weiterer Forschungsbedarf.

Der mögliche Temperatureinfluss auf den Abbau von Tetrazyklin in Masthähnchenfestmist sollte in zukünftigen Arbeiten unter besonderer Beachtung der an der Kompostierung beteiligten Mikroorganismen und deren mögliche Hemmung durch Tetrazyklin untersucht werden. Auch darüber hinaus gehende Versuche zur Wirkung von Antibiotika auf Methanbakterien bei der anaeroben Vergärung könnten über das mögliche Schadstoffpotential von Tetrazyklinen und anderen Antibiotika aus der Landwirtschaft weiteren Aufschluss geben.

Belastbarere Aussagen über die Mobilität bzw. die Immobilität von Tetrazyklinen und Sulfonamiden in verschiedenen Böden erfordern weitere Versuche unter Feldbedingungen, denn seit mehreren Jahren befüllte Lysimeter stellen anthropogen beeinflusste Böden dar, die Versuche unter Feldbedingungen nicht ersetzen können Der potenzielle Einfluss der Einarbeitungsform der Gülle auf die Verlagerungsneigung von Tetrazyklin und auch die Fruchtart sollte zusätzlich Berücksichtigung finden. 


\section{Zusammenfassung}

Antibiotika, vor allem Tetrazykline und Sulfonamide, werden in nennenswertem Umfang für veterinärmedizinische Zwecke in der Nutztierhaltung eingesetzt. So betrug 1999 der Antibiotika-Verbrauch in der EU sowie in der Schweiz 13.200 t.

Nach Verabreichung werden Arzneimittel in nach Wirkstoff und Tierart unterschiedlicher Menge und Form durch den tierischen Organismus wieder ausgeschieden. Daher ist in dem Pfad Exkretion-Wirtschaftsdünger-Boden eine bedeutende Eintragsquelle potenziell umweltrelevanter Stoffe zu sehen. Während alle neu zugelassenen Wirkstoffe seit 1998 einer Umweltverträglichkeitsprüfung unterliegen (EMEA/CVMP-055/96), gilt dies nicht für sogenannte Altpräparate wie Tetrazykline und Sulfonamide. Daher besteht ein erheblicher Mangel an Informationen über das Verhalten von diesen Altpräparaten in der Umwelt.

Ziel dieser Arbeit war es daher, in drei Themenschwerpunkten die Wirkstoffgruppen Tetrazykline und Sulfonamide hinsichtlich ihres Umweltverhaltens in Masthähnchenfestmist (Persistenz von Tetrazyklin), Schweinegülle (Erhebung zu tatsächlich vorliegenden Wirkstoffgehalten in der Praxis) und Boden (Mobilität von Tetrazyklin und Sulfadiazin in verschiedenen Böden) zu untersuchen und damit einen Beitrag zur Risikobewertung dieser Antibiotika zu leisten. Die Quantifizierung der Substanzen erfolgte über HPLC-gestützte Nachweisverfahren.

In zwei Versuchsdurchgängen zur Persistenz von Tetrazyklin in Masthähnchenfestmist fand der stärkste Wirkstoffabbau in den ersten 14 Tagen nach Aufsetzen des Festmistes statt. In dieser Zeit erfolgte ein Temperaturanstieg auf etwa $55^{\circ} \mathrm{C}$ bzw. $48{ }^{\circ} \mathrm{C}$. Im weiteren Verlauf der Lagerung gingen die Tetrazyklin-Konzentrationen beider Lagerungsvarianten weiter zurück, ein vollständiger Abbau innerhalb der Lagerungsdauer von drei bzw. zwei Monaten wurde in beiden Experimenten nicht erzielt. Es ließ sich kein eindeutiger Unterschied hinsichtlich Temperaturverlauf oder Tetrazyklinabbau zwischen den Lagerungsarten Rotte- bzw. Stapelmist nachweisen. Die Temperaturen in den unbelasteten Kontrollen lagen um bis zu 3 Kelvin deutlich über jenen der mit Tetrazyklin versetzten Varianten, was auf eine Hemmung der an der Kompostierung beteiligten Mikroorganismen durch Tetrazyklin hindeutet. Die Halbwertszeiten für Tetrazyklin betrugen 2,9 Tage in Rotte- und 2,3 Tage in Stapelmist. Diese Angaben sind allerdings nur als 
Orientierungswerte zu betrachten, da aufgrund des sehr heterogenen Probenmaterials große Schwankungen in den Messwerten auftraten.

Für das Screening von Schweinegüllen standen zwei Probensätze aus den Jahren 1999/2000 ( $\mathrm{n}=168)$ und 2001/2002 ( $\mathrm{n}=176)$ aus der Region Weser-Ems zur Verfügung. 1999/2000 wurden 35,1\% der Proben negativ auf die untersuchten Antibiotika (Tetrazyklin, Oxytetrazyklin, Chlortetrazyklin, Sulfadiazin, Sulfamethazin) getestet. Die am häufigsten nachweisbaren Wirkstoffe waren Tetrazyklin und Sulfamethazin (38,7 bzw. 57,1 \% der Proben). 2001/2002 stellten Tetrazyklin und Sulfamethazin mit jeweils fast $50 \%$ die am stärksten vertretenen Antibiotika dar, 12,5 \% der Proben waren ohne Befund. Eine Änderung der tierärztlichen Verschreibungspraxis fand offensichtlich für Sulfadiazin statt (1999/2000: 8,3\%; 2001/2002: 48,9\%). Die ermittelten Sulfonamidkonzentrationen unterschritten trotz vergleichbarer empfohlener Dosierung deutlich die Werte für Tetrazykline; dies deutet auf eine geringere Persistenz der Sulfonamide in Gülle hin. Insgesamt belegen die vorliegenden Ergebnisse den verbreiteten Einsatz von Antibiotika in der Schweinehaltung; die longitudinale Betrachtung ließ keine Reduzierung des Antibiotikaeinsatzes erkennen.

Ein Teil der Untersuchungen zur Mobilität von Tetrazyklin und Sulfadiazin in verschiedenen Böden wurde in Kick-Brauckmann-Gefäßen mittels Starkregensimulation zu unterschiedlichen Zeitpunkten durchgeführt. Bei Beregnung $48 \mathrm{~h}$ nach Aufbringung tierarzneimittelhaltiger Gülle konnten im Sickerwasser aus humosem Sandboden sowie lehmigem Schluff weder Tetrazyklin noch Sulfadiazin oberhalb der Bestimmungsgrenze von 2 bzw. $6 \mu \mathrm{g} \mathrm{l}^{-1}$ nachgewiesen werden. In zwei von drei Gefäßen mit sogenannter Nullerde (Torfgemisch) lag dagegen in allen Sickerwasserfraktionen Tetrazyklin in Spuren vor (1,6 - 8,2 $\left.\mu \mathrm{g} \mathrm{l}^{-1}\right)$. Bei Beregnung nach zehn Wochen konnten dagegen unabhängig von der Bodenart weder Tetrazyklin noch Sulfadiazin im Sickerwasser nachgewiesen werden.

Im Oberboden der Kick-Brauckmann-Gefäße lagen nach dem frühen Starkregenereignis generell höhere Tetrazyklinmengen vor als im Unterboden. Die Gehalte schwankten jedoch sehr stark zwischen den Bodenarten und waren offensichtlich vom Tongehalt des Bodens abhängig (lehmiger Schluff $>$ humoser Sand $>$ Nullerde). Grundsätzlich lagen die Sulfadiazin-Konzentrationen $48 \mathrm{~h}$ nach Gülleaufbringung bei gleicher Applikationsmenge um ein Vielfaches unter denen des Tetrazyklins. Nach zehn Wochen waren für beide Wirkstoffe deutlich geringere Konzentrationen im Boden nachweisbar Insgesamt belegen die Ergebnisse, dass Sulfadiazin im Boden sehr viel weniger stabil ist als Tetrazyklin. 
Tetrazyklin zeigte zumindest in sandigen und lehmigen Böden eine ausgeprägte Stabilität sowie eine geringe Verlagerungsneigung. Eine höhere Verlagerungsneigung in strukturreichen Moorböden, eventuell gebunden an Güllebestandteile, kann nicht ausgeschlossen werden.

Längerfristige Untersuchungen wurden mittels mit Mais bzw. Roggen bestellten Krumenlysimetern durchgeführt, deren Düngung über antibiotikahaltige Gülle erfolgte. Im ersten Untersuchungsjahr wurde in vier Sickerwasserproben unter Mais Tetrazyklin in einer Konzentration von 0,9 bis $3,1 \mu \mathrm{g}^{-1}$ nachgewiesen (Bestimmungsgrenze $1 \mu \mathrm{g}^{-1}$ )) nachgewiesen. Die positiven Tetrazyklin-Befunde traten in den ersten vier Wochen nach Gülleapplikation bei relativ geringem Sickerwasseranfall auf. Dieser Befund ließ sich im zweiten Untersuchungsjahr jedoch nicht reproduzieren. Im zweiten Versuchsjahr zusätzlich eingesetztes Sulfadiazin war weder unter Mais noch unter Roggen nachweisbar.

Damit liegen zumindest für das (anthropogen beeinflusste) Lysimetermodell erstmals Hinweise auf eine Verlagerbarkeit von Tetrazyklin vor. Es war keine Dosisabhängigkeit für das Auftreten oder die Höhe der Auswaschung ableitbar. Dagegen könnte ein Zusammenhang mit der Aufbringung bzw. Einarbeitung der wirkstoffhaltigen Gülle bestehen (Mais: ca. 4 cm tiefe Einarbeitung; Roggen: oberflächliche Aufbringung).

Die wiederholte Untersuchung des Lysimeterbodens auf Tetrazyklin ergab über die beiden Untersuchungsjahre einen Anstieg des Wirkstoffgehaltes im Boden. Sulfadiazin erreichte nach Applikation über Gülle wesentlich geringere Konzentrationen im Boden als Tetrazyklin und wurde über den Probennahmezeitraum stark abgebaut.

Die Versuche zur Mobilität von Tetrazyklin und Sulfadiazin in verschiedenen Böden haben lediglich Modellcharakter. Vor diesem Hintergrund ist das Auswaschungsrisiko unter Praxisbedingungen als sehr gering einzuschätzen. 


\section{Summary}

Veterinary antibiotics, mainly tetracyclines and sulphonamides, are commonly used in intensive livestock production. In 1999, the use of antibiotics in the EU and Switzerland amounted to $13.200 \mathrm{t}$.

After administration to the animal, considerable amounts of the agents or their metabolites are excreted via urine and faeces. Through organic fertilizers biologically active ingredients may reach the soil and become environmentally relevant. According to a guideline which has been developed by the European Medicines Agency (EMEA/CVMP055/96) and came into effect in 1998, an environmental impact assessment is obligatory for all new veterinary drugs. However, this procedure is not required for so-called old compounds such as tetracyclines and sulphonamides. Therefore, a substantial lack of information exists for these old compounds with regard to potential environmental effects.

The aim of this thesis was to examine the substance groups tetracyclines and sulphonamides with regard to their behaviour in poultry manure (persistence of tetracycline), liquid manure (drug residues in samples from commercial farms) and soil (mobility of tetracycline and sulfadiazine in different soils) with the final aim to provide further information for the risk assessment of these antibiotics. The detection of the substances was carried out using HPLC.

In two experiments with stored poultry manure containing tetracycline the strongest degradation took place during the first 14 days after set-up of the containers. During this period, a considerable temperature rise to approximately $55^{\circ} \mathrm{C}$ in the first and $48{ }^{\circ} \mathrm{C}$ in the second experiment was observed thus indicating a temperature dependency in the degradation of tetracycline. In the further course of the storage period tetracycline concentrations of both storage types (aerobic and anaerobic conditions) were further reduced. However, in both experiments an entire degradation was not achieved within the storage period of three and two months, respectively. Temperature levels in the controls remained above the tetracycline treated containers ( 3 Kelvin) indicating a tetracyclineinduced inhibition of microorganisms involved in the composting process. Half-lifes for tetracycline $2.9 \mathrm{~d}$ for more aerobic and $2.3 \mathrm{~d}$ for more anaerobic storage conditions. However, a large variability in concentrations due to the heterogenous nature of the samples has to be taken into account. 
In two batches of liquid manure samples originating from the Weser-Ems region (1999/2000: $n=168 ; 2001 / 2002: n=176)$, the occurrence of five commonly used antibiotics was investigated. In $1999 / 2000,35.1 \%$ of the samples were negative for the examined antibiotics (tetracycline, oxytetracycline, chlortetracycline, sulfadiazine, sulfamethazine), whereas this was the case for $12.5 \%$ of the samples in 2001/2002. In both years, the most frequently detected substances were tetracycline and sulfamethazine. However, sulphonamides seem to be less persistent in liquid manure compared to tetracyclines. In general, the present results demonstrate the widespread use of antibiotics in pig production, and a longitudinal analysis provided no evidence for a substantial reduction in the use of the antibiotics investigated.

The investigations on the mobility of tetracycline and sulfadiazine in different soils were partly carried out using Kick-Brauckmann vessels and intense rain simulation at different time points. Neither tetracycline nor sulfadiazine were detected in the leachate from humous sandy soil as well as from loamy sand when irrigation took place $48 \mathrm{~h}$ after application of the antibiotics with liquid manure (quantification limit: $2-6 \mu \mathrm{g}^{-1}$ ). However, in two of three vessels with peat-based substrate tetracycline was detected in all four leachate samples in a range from 1.6 to $8.2 \mu \mathrm{g} \mathrm{l}^{-1}$. Irrigation after ten weeks did not reveal any detectable traces in the leachates irrespective of the soil type. Thus, a higher leaching potential of tetracycline in boggy soil, possibly bound to liquid manure particles, cannot be excluded.

In long-term lysimeter studies, tetracycline was detected in four leachate samples under corn (quantification limit $1 \mu \mathrm{g}^{-1}$ ) in the first year. However, these findings could not be reproduced in the second year. Sulfadiazine, which had only been used in the second year, was neither detected under corn nor under rye. These results report for the first time a leaching potential of tetracycline at least for the lysimeter model. Leaching did not depend on the amount of tetracycline applied with liquid manure. However, it could be connected with the type of application or incorporation into the soil of the liquid manure, respectively (rye: superficial application, corn: incorporation at a depth of $4 \mathrm{~cm}$ ).

In the top soil layer of the tested Kick-Brauckmann vessels a higher tetracycline concentration was generally found than in the lower soil layer. Concentrations were obviously dependent on the clay content of the soil (loamy sand $>$ humous sand $>$ peat mixture). Although applied in the same amount, soil sulfadiazine concentrations were always lower than those of tetracycline; this was also true for the lysimeter experiments. 
After ten weeks the concentration of both substances had clearly decreased. However, repeated analysis of the soil from the lysimeters showed an increase of tetracycline concentrations over the two years. This points to an accumulation risk for tetracycline in soil since the substance showed at least in sandy and loamy soil a distinct stability in the soil as well as a low leaching ability. 


\section{Literatur}

ABBAS, B.; LINKE, I.; KRATZ, W. (2001): Erhebung des Verbrauchs von Arzneimittelwirkstoffen im Land Brandenburg. Zeitung für Umweltchemie und Ökotoxikologie 13 (4), 197-203.

AL-AHMAD, A.; DASCHNER, F. D.; KÜMMERER, K. (1999): Biodegradability of cefotiam, ciprofloxacin, meropenem, penicillin $\mathrm{G}$, and sulfamethoxazole and inhibition of waste water bacteria. Arch. Environ. Contam. Toxic. 37, 158-163.

ANONYMOUS (2003): Leistungsförderer Avilamycin bleibt für Puten erhalten. Animal Health Online, elektronische Mitteilung.

ARBEITSGEMEINSCHAFT DEUTSCHER TIERZÜCHTER (2001): Antibiotikaeinsatz in der Tierhaltung minimieren. Animal Health Online, elektronische Mitteilung.

BAGUER, A. J.; JENSEN, J.; KROGH, P. H. (2000): Effects of the antibiotics oxytetracycline and tylosin on soil fauna. Chemosphere 40, 751-757.

BATCHELDER, A. R. (1981): Chlortetracycline and oxytetracycline effects on plant growth and development in liquid cultures. Journal of Environmental Quality 10, 515-518.

BATCHELDER, A. R. (1982): Chlortetracycline and oxytetracycline effects on plant growth and development in soil systems. Journal of Environmental Quality 11, 675678.

BAYERISCHES LANDESAMT FÜR UMWELTSCHUTZ (1999): Die Eigenkompostierung - Eine Betrachtung unter hygienischen Aspekten. München.

BERGER, K.; PETERSEN, B.; BÜNING-PFAUE, H. (1986): Persistenz von GülleArzneistoffen in der Nahrungskette. Archiv für Lebensmittelhygiene 37, 99-102.

BGVV (1995): Nachweismethode für Rückstände von Tierarzneimitteln: Bestimmung der Rückstände von Oxytetrazyklin, Tetrazyklin und Chlortetrazyklin in essbarem Gewebe von Rind, Schaf, Schwein, Pute, Forelle, Karpfen sowie Kuhmilch und Hühnerei (Routineverfahren). Bundesgesundheitsblatt 10/95.

BILITEWSKI, B.; HÄRDTLE, G.; MAREK, K. (1990): Abfallwirtschaft. Springer Verlag, Berlin.

BOXALL, A. B. A.; BLACKWELL, P.; CAVALLO, R.; KAY, P.; TOLLS, J. (2002): The sorption and transport of a sulphonamide antibiotic in soil systems. Toxicology Letters 131, 19-28.

BRÜGGEMANN, J.; LÖSCH, U.; MERKENSCHLAGER, M.; OFFTERDINGER, I. (1966): Ablagerung von Tetrazyklin im Knochengewebe von Tieren bei dem Zusatz von Tetrazyklin zum Futter. Zentralblatt für Veterinärmedizin 13, 59-74. 
CAMPAGNOLO, E. R.; JOHNSON, K. R.; KARPATI, A.; RUBIN, C. S.; KOLPIN, D. W.; MEYER, M. T.; ESTEBAN, J. E.; CURRIER, R. W.; SMITH, K.; THUG, K. M.; McGEEHIN, M. (2003): Antimicrobial residues in animal waste and water resources proximal to large-scale swine and poultry feeding operations. Science of the Total Environment 299, 89-95.

CHEVITA (2004): schriftliche Mitteilung.

CHRISTIAN, T.; SCHNEIDER, R. J.; FÄRBER, H. A.; SKUTLAREK, D.; MEYER, M. T.; GOLDBACH, H. E. (2003): Determination of antibiotic residues in manure, soil, and surface waters. Acta hydrochim. hydrobio. 31, 45-48.

DAUGHTON, C. G.; TERNES, T. A. (1999): Pharmaceuticals and personal care products in the environment: agents of subtle change? Environmental Health Perspectives 107, 907-938.

DE LIGUORO, M.; CIBIN, V.; CAPOLONGO, F.; HALLING-SØRENSEN; MONTESISSA, C. (2003): Use of oxytetracycline and tylosin in intensive calf farming: evaluation of transfer to manure and soil. Chemosphere 52, 203-212.

DÜNGE-VO (1996): BGBI I, 118.

ELMUND, G. K.; MORRISON, S. M.; GRANT, D. W.; NEVINS, M. P. (1971): Role of excreted chlortetracycline in modifying the decomposition process in feedlot waste. Bull. Environ. Contamin. Toxic. 6, 129-132.

EMEA/CVMP (2000): Guideline on environmental impact assessment for veterinary products CVMP/VICH/592/98. London, United Kingdom.

EU-KOMMISSION (KOM) (2002): Vorschlag für eine Verordnung des Europäischen Parlaments und des Rates über Zusatzstoffe zur Verwendung in der Tierernährung. 153 endg.; Ratsdokument 7505/02, Drucksache 340/02.

FEDESA (1999): Antibiotics for animals: A FEDESA perspective on antibiotics, animal health and the resistance debate. www.fedesa.be.

FEDESA (2001): Antibiotic use in farm animals does not threaten human health, a new study suggests.www.fedesa.be/Pressroom/PR-130601.htm.

FEDLER, C. B.; DAY, D. L. (1985): Anaerobic digestion of swine manure containing an antibiotic inhibitor. Agric. Waste Utiliz. Manag., 523-530.

GAVALCHIN, J.; KATZ, S. E. (1994): The persistence of fecal-borne antibiotics in soil. Journal of AOAC International 77 (2), 481-485.

GRAFE, A. (2001): Untersuchungen zum Einsatz pharmakologisch wirksamer Stoffe in der Veredelungswirtschaft unter besonderer Berücksichtigung der Tetrazykline. Göttingen: Cuvillier Verlag Göttingen. Dissertation Universität Göttingen. 1-157.

GRUBER, V. F.; HALleY, B. A.; HWANG, S.-C.; KU, C. C. (1990): Mobility of avermectin $\mathrm{B}_{1 \mathrm{a}}$ in soil. Journal of Agriculture and Food Chemistry 38, 886-890. 
GUHDE, I. (1998): Methodenentwicklung und Validierung in der HPLC nach Festphasenextraktion. Dissertation. Universität Würzburg.

HALlER, M. Y.; MÜlleR, S.; McARDELl, C. S.; ALDER, A. C.; SUTER, M. J.-F. (2002): Quantification of veterinary antibiotics (sulfonamides and trimethoprim) in animal manure by liquid chromatography-mass spectrometry. Journal of Chromatography A 952 (1-2), 111-120.

HALLING-SØRENSEN, B. (2000): Algal toxicity of antibacterial agents used in intensive farming. Chemosphere 40, 731-739.

HALLING-SØRENSEN, B.; SENGELØV, G.; TJØRNELUND，J. (2003): Reduced antimicrobial potencies of oxytetracycline, tylosin, sulfadiazin, streptomycin, ciprofloxacin, and olaquindox due to environmental processes. Archive of Environmental Contamination and Toxicology 44, 7-16.

HAMSCHER, G.; SCZESNY, S.; ABU-QUARE, A.; HÖPER, H.; NAU, H. (2000): Stoffe mit pharmakologischer Wirkung einschließlich hormonell aktiver Substanzen in der Umwelt: Nachweis von Tetrazyklinen in güllegedüngten Böden. Deutsche tierärztliche Wochenschrift 107 (8), 332-334.

HAMSCHER, G.; SCZESNY, S..; HÖPER, H; NAU, H. (2002): Determination of persistent tetracycline residues in soil fertilized with liquid manure by high performance liquid chromatography with electrospray ionization tandem mass spectrometry. Analytical Chemistry 74,1509-1518.

HAMSCHER, G.; PAWELZICK; H. T.; SCZESNY, S.; NAU, H.; HARTUNG, J. (2003): Antibiotics in dust originating from a pig-fattening farm: a new source of health hazard for farmers?. Environmental Health Perspectives 111 (13), 1590-1594.

HANSEN, L. H.; AARESTRUP, F.; SØRENSEN, S. J. (2002): Quantification of bioavailable chlortetracycline in pig feces using a bacterial whole-cell biosensor. Veterinary Microbiology 87, 51-57.

HARTIG, C.; STORM, T.; JEKEL, M. (1999): Detection and identification of sulphonamide drugs in municipal waste water by liquid chromatography coupled with electrospray ionisation tandem mass spectrometry. Journal of Chromatography A 854, 163-173.

HEBERER, T.; STAN, H.-J. (1998): Arzneimittelrückstände im aquatischen System. Wasser Boden 50, 20-25.

HESKETH, N.; BROOKES, P. C.; ADDISCOTT, T. M. (2001): Effect of suspended soil material and pig slurry on the facilitated transport of pesticides, phosphate and bromide in sandy soil. European Journal of Soil Science 52 (2), 287-296.

HILPERT, R.; WINTER, J.; KANDLER, O. (1984): Agricultural feed additives and disinfectants as inhibitory factors in anaerobic digestions. Agricultural Wastes 10, 103-116. 
HIRSCH, R.; TERNES, T.; HABERER, K.; MEHLICH, A.; BALLWANZ, A.; KRATZ, K. L. (1998): Determination of antibiotics in different water compartments via liquid chromatography-electrospray tandem mass spectrometry. Journal of Chromatography A 815, 213-223.

HOLTEN LÜTZHØFT, H. C.; VAES, W. H. J.; FREIDIG, A. P.; HALLINGSØRENSEN, B.; HERMENS, J. L. M. (2000): Influence of pH and other modifying factors on the distribution behaviour of 4quinolones to solid phases and humic acids studied by 'negligible-depletion' SPME-HPLC. Environmental Science and Technology 34, 4989-4994.

HÖPER, H.; KUES, J.; NAU, H.; HAMSCHER, G. (2002): Eintrag und Verbleib von Tierarzneimittelwirkstoffen in Böden. Bodenschutz 4, 141-148.

INGERSLEV, F.; HALLING-SØRENSEN, B. (2000): Biodegradability properties of sulfonamides in activated sludge. Environmental Toxicology and Chemistry 19, $2467-2473$

JAGNOW, G. (1977): Mikrobieller Abbau der Futtermittelantibiotika Zink-Bacitracin, Flavophospholipol, Spiramycin und von Tetrazyklin in feucht gelagertem und in mit Boden vermischtem Hühnerkot. Landwirtschaftliche Forschungsanstalt, Kongressband, Teil 1 (1), 227-234.

JJEMBA, P. K. (2002): The potential impact of veterinary and human therapeutic agents in manure and biosolids on plants grown on arable land: a review. Agriculture, Ecosystems and Environment 93, 267-278.

KERN, A.; SCHULTZE, B.; DURNER, W. (2003): Einfluss von Bodenbearbeitungsmaßnahmen auf die Ausprägung des präferentiellen Stofftransports in einem Boden unter Abwasserverregnung. Mitteilungen der Bodenkundlichen Gesellschaft 96 (2), 95-96.

KOLPIN, D. W.; FURLONG, E. T.; MEYER, M. T.; THURMAN, E. M.; ZAUGG, S. D.; BARBER, L. B.; BUXTON, H. T. (2002): Pharmaceuticals, hormones, and other organic wastewater contaminants in US streams, 1999-2000: A national reconnaissance. Environmental Science and Technology 36, 1202-1211.

KROKER, R. (1983): Aspekte zur Ausscheidung antimikrobiell wirksamer Substanzen nach der chemotherapeutischen Behandlung von Nutztieren. Wissenschaft und Umwelt 4, 305-308.

KROKER, R.; LÖSCHER, W.; ŠIMŮNEK, J.; TROLLDENIER, H.; UNGEMACH, F. R. (1996): Chemotherapie bakterieller Infektionen. In: Frey, H-H und Löscher, W. (Hrsg.): Lehrbuch der Pharmakologie und Toxikologie für die Veterinärmedizin. Enke, Stuttgart.

KROKER, R. (1997): Pharmaka zur Behandlung und Verhütung bakterieller Infektionen. In: Löscher, W. et al. (Hrsg.): Pharmakotherapie bei Haus- und Nutztieren. 3. Auflage, Parey, Berlin.

KROKER, R. (2002): Kompaktwissen Tierarzneimittelrückstände. B. Behr's, 1. Auflage, Hamburg. 
KÜHNE, M., IHNEN, D.; MÖLLER, G.; AGTHE, O. (2000): Stability of tetracycline in water and liquid manure. Journal of Veterinary Medicine A 47, 379-384.

KÜHNE, M.; HAMSCHER, G.; KÖRNER, U.; SCHEDL, D.; WENZEL, S. (2001): Formation of anhydrotetracycline during a high-temperature treatment of animalderived feed contaminated with tetracycline. Food Chemistry 75, 423-429.

LANGHAMMER, J.-P. (1989): Untersuchungen zum Verbleib antimikrobiell wirksamer Arzneistoffe als Rückstände in Gülle und im landwirtschaftlichen Umfeld Dissertation Universität Bonn, 1-138.

LINDSEY, M. E.; MEYER, M.; THURMAN, E. M. (2001): Analysis of trace levels of sulfonamide and tetracycline antimicrobials in groundwater and surface water using solid-phase extraction and liquid chromatography/mass spectrometry. Analytical Chemistry 73 (19), 4640-4646.

LOKE, M. L.; TJØRNELUND, J.; HALLING-SØRENSEN, B. (2002): Determination of the distribution coefficient $\left(\log \mathrm{K}_{\mathrm{d}}\right)$ of oxytetracycline, tylosin $\mathrm{A}$, olaquindox, and metronidazole in manure. Chemosphere 48, 351-361.

MASSÉ, D. I.; LU, D.; MASSE, L.; DROSTE, R. L. (2000) : Effect of antibiotics on psychrophilic anaerobic digestion of swine manure slurry in sequencing batch reactors. Bioresource Technology 75, 205-211.

McCOY, R. E. (1976): Uptake, translocation, and persistence of oxytetracycline in coconut palm. Phytopathology 66, 1038-1042.

McGECHAN, M. B.; LEWIS, D. R. (2002): Transport of particulate and colloid-sorbed contaminants through soil, part 1: General principles. Biosystems Engineering 83 (3), 255-273.

McGRATH, J. W.; HAMMERSCHMIDT, F.; QUINN, J. P. (1998): Biodegradation of phosphonomycin by Rhizobium huakuii PMY1. Applied Environmental Microbiology 64, 356-358.

McMANUS, P. S.; STOCKWELL, V. O.; SUNDIN, G. W.; JONES; A. L. (2002): Antibiotic use in plant agriculture. Annual Review Phytopathology 40, 443-465.

MIGLIORE, L.; BRAMBILLA, G.; COZZOLINO, S.; GAUDIO, L. (1995): Effect on plants of sulphadimethoxine used in intensive farming (Panicum miliaceum, Pisum sativum and Zea mays). Agriculture, Ecosystems and Environment 52, 103-110.

MONTFORTS, M. H. M. M.; KALF, D. F.; VLAARDINGEN VAN, P. L. A.; LINDERS, J. B. H. J. (1999): The exposure assessment for veterinary medicinal products. The Science of the Total Environment 225, 119-133.

PATTEN, D. K.; WOLF, D. C.; KUNKLE, W. E.; DOUGLASS, L. W. (1980): Effect of antibiotics in beef cattle feces on nitrogen and carbon mineralization in soil and on plant growth and composition. Journal of Environmental Quality 9, 167-172. 
PFEIFER, T.; TUERK, J.; BESTER, K.; SPITELLER, M. (2002): Determination of selected sulfonamide antibiotics and trimethoprim in manure by electrospray and atmospheric pressure chemical ionization tandem mass spectrometry. Rapid Communications in Mass Spectrometry 16, 663-669.

POELS, J.; VAN ASSCHE, P.; VERSTRAETE, W. (1984): Effects of disinfectants and antibiotics on the anaerobic digestion of piggery waste. Agricultural Wastes 9, 239247.

RABØLLE, M.; SPLIID, N. H. (2000): Sorption and mobility of metronidazole, olaquindox, oxytetracycline and tylosin in soil. Chemosphere 40, 715-722.

RAHE, T. M.; HGEDORN, C.; McCOY, E. L.; KLING, G. F. (1978): Transport of antibiotic Escherichia Coli through western Oregon hillslope soils under conditions of saturated flow. Journal of Environmental Quality 7, 487-494.

RASSOW, D.; SCHAPER, H. (1996): Zum Einsatz von Fütterungsarzneimitteln in Schweine- und Geflügelbeständen in der Region Weser-Ems. Deutsche tierärztliche Wochenschrift 103 (7), 244-249.

RÖMPP (1995): Römpp Chemie Lexikon (CD). Version 1.0. Georg Thieme Verlag, Stuttgart, New York.

ROTH, S. (1994): Mikrobiologisch-hygienische Untersuchungen zur Bioabfallkompostierung in Mieten und Kleinkompostern. Dissertation, Universität Hohenheim.

SAMUELSEN, O. B.; LUNESTAD, B. T.; ERVIK, A.; FJELDE, S. (1994): Stability of antibacterial agents in an artificial marine aquaculture sediment studied under laboratory conditions. Aquaculture 126, 283-290.

SANKVIST, A.; HAGELBERG, M.; MATHISEN, B. (1984): Effects of antibiotics and chemotherapeutics on biogas production from piggery waste. Bioenergy 84, 422-426.

SCHAPER, R.; LIEBISCH, A. (1991): Einfluss eines systemisch wirkenden Antiparasitikums (Ivermectin) auf die Dungfauna und den Dungabbau der Rinder in Weidehaltung. Tierärztliche Umschau 46, 12-18.

SCHEFFER, F.; SCHACHTSCHABEL, P. (1992): Lehrbuch der Bodenkunde. 13. Auflage, Enke, Stuttgart.

SCHÜLLER, S. (1998): Anwendung antibiotisch wirksamer Substanzen beim Tier und Beurteilung der Umweltsicherheit entsprechender Produkte. 3. Statuskolloquium ökotoxikologischer Forschungen in der Euregio Bodensee, 3.- 4. Dezember 1998.

SENGELØV, G.; AGERSO, Y.; HALLING-SØRENSEN, B.; BALODA, S. B.; ANDERSEN, J. S.; JENSEN, L. B. (2003): Bacterial antibiotic resistance levels in Danish farmland as a result of treatment with pig manure slurry. Environment International 28, 587-595.

SITHOLE, B. B.; GUY, R. D. (1987): Models for oxytetracycline in aquatic environments. Interactions with humic substances. Water, Air and Soil Pollution 32, 315-321. 
STAATLICHES LEBENSMITTELUNTERSUCHUNGSAMT OLDENBURG (1999): Bestimmung von Oxytetrazyklin, Tetrazyklin, Chlortetrazyklin und deren 4Epimere in Lebensmitteln tierischer Herkunft.

STAHEL, W. A. (2000): Statistische Datenanalyse - Eine Einführung für Naturwissenschaftler. 3. Auflage, Vieweg, Braunschweig, Wiesbaden.

STREBE, T. A.; TALBERT, R. E. (2001): Sorption and mobility of flumetsulam in several soils. Weed Science 49, 806-813.

STRONG, L.; WALL, R.; WOOLFORD, A.; DJEDDOUR, D. (1996): The effect of faecally excreted ivermectin and fenbendazole on the insect colonisation of cattle dung following the oral administration of sustained-release boluses. Veterinary Parasitology 62, 252-266.

TERNES, T. A. (2001): Analytical methods for the determination of pharmaceuticals in aqueous environmental samples. Trends in Analytical Chemistry 20 (8), 419-434.

THIEHLE-BRUHN, S. (2003a): Antibiotika. In: Handbuch der Bodenkunde, 15. Erg. Lfg. 5/03. ecomed-Verlag.

THIELE-BRUHN, S. (2003b): Pharmaceutical antibiotic compounds in soils - a review. Journal of Plant Nutrition and Soil Science 166, 145-167.

THIELE-BRUHN, S.; MOGK, A.; FREITAG, D. (2003): Einsatz von Tierarzneimitteln zur Anwendung bei landwirtschaftlichen Nutztieren. Berichte über Landwirtschaft 81 (3), 374-391.

THOMAS, G. K.; MILLAR, R. G.; ANSTIS, P. W. (1997): Stability of sulfonamide antibiotics in spiked pig liver tissue during frozen storage Journal of AOAC International 80 (5), 988-995.

TOLLS, J. (2001): Sorption of veterinary pharmaceuticals in soils: A review. Environmental Science and Technology 35, 17, 3397-3406.

WALL, R.; STRONG, L. (1987): Environmental consequences of treating cattle with the antiparasitic drug ivermectin. Nature 327, 418-421.

WARMAN, P. R.; THOMAS, R. L. (1980): Chlortetracycline in soil amended with poultry manure. Canadian Journal of Soil Science 61, 161-163.

WATTS, C. D.; CRATHORNE, B.; FIELDING, M.; KILLOPS, S. D. (1982): Non-volatile organic-compounds in treated waters. Environmental Health Perspectives 46, 87-99.

WINCKLER, C.; GRAFE, A. (2000): Charakterisierung und Verwertung von Abfällen aus der Massentierhaltung unter Berücksichtigung verschiedener Böden. UBA-Text 44/00. Umweltbundesamt. Umweltforschungsplan des Bundesministeriums für Umwelt, Naturschutz und Reaktorsicherheit Forschungsbericht 29733911, 1-145.

ZAIED, H. (1999): Untersuchungen zum Einfluss verschiedener Zusätze auf den Rotteverlauf, die Stickstoffdynamik und die Kompostqualität bei der Kompostierung von Reststoffen aus der Legehennenhaltung. Dissertation, Universität Göttingen. 
ZHU, J.; SNOW, D. D.; CASSADA, D. A.; MONSON, S. J.; SPALDING, R. F. (2001): Analysis of oxytetracycline, tetracycline and chlortetracycline in water using solid phase extraction and liquid chromatography-tandem mass spectrometry. Journal of Chromatography A 928, 177-186. 


\section{Anhang}

Tabelle 34: Tagesmittelwerte des Temperaturverlaufs im Masthähnchenfestmist der sechs Komposter und der Raumluft, Experiment 1

\begin{tabular}{|c|c|c|c|c|c|c|c|}
\hline \multirow[t]{2}{*}{ Tage } & \multicolumn{7}{|c|}{ Temperatur $\left({ }^{\circ} \mathrm{C}\right)$} \\
\hline & $\begin{array}{c}0,5 \text { g TC je } \\
\text { kg Futter } \\
\text { RM }\end{array}$ & $\begin{array}{l}\text { KT } \\
\text { RM }\end{array}$ & $\begin{array}{c}0,5 \text { g TC je } \\
\text { kg Futter } \\
\text { SM }\end{array}$ & $\begin{array}{c}1,0 \text { g TC je } \\
\text { kg Futter } \\
\text { RM }\end{array}$ & $\begin{array}{l}\text { KT } \\
\text { SM }\end{array}$ & $\begin{array}{c}1,0 \text { g TC je } \\
\text { kg Futter } \\
\text { SM }\end{array}$ & Raumluft \\
\hline 1 & 22,1 & 22,7 & 23,3 & 26,1 & 23,0 & 25,8 & 16,5 \\
\hline 2 & 45,4 & 44,9 & 44,2 & 46,6 & 44,8 & 44,7 & 16,3 \\
\hline 3 & 53,4 & 54,3 & 54,5 & 50,7 & 56,0 & 47,6 & 16,5 \\
\hline 4 & 53,9 & 55,5 & 55,3 & 48,0 & 57,7 & 45,8 & 16,3 \\
\hline 5 & 53,7 & 55,5 & 54,5 & 46,5 & 56,9 & 45,5 & 16,4 \\
\hline 6 & 54,7 & 55,9 & 53,6 & 49,5 & 56,3 & 48,6 & 16,3 \\
\hline 7 & 56,3 & 56,5 & 53,6 & 54,1 & 56,8 & 52,7 & 16,5 \\
\hline 8 & 56,6 & 56,3 & 54,2 & 55,1 & 57,4 & 53,5 & 16,5 \\
\hline 9 & 55,1 & 54,1 & 55,3 & 54,3 & 56,6 & 52,3 & 16,4 \\
\hline 10 & 54,7 & 52,9 & 52,1 & 54,4 & 55,5 & 51,7 & 16,6 \\
\hline 11 & 55,0 & 53,7 & 51,5 & 54,6 & 55,1 & 51,6 & 16,1 \\
\hline 12 & 53,9 & 53,7 & 50,5 & 53,0 & 54,5 & 50,9 & 15,6 \\
\hline 13 & 52,8 & 52,6 & 49,6 & 52,8 & 53,9 & 50,3 & 15,3 \\
\hline 14 & 52,3 & 51,0 & 49,0 & 53,2 & 53,5 & 49,6 & 15,5 \\
\hline 15 & 52,2 & 49,7 & 48,9 & 53,0 & 53,2 & 48,9 & 15,6 \\
\hline 16 & 52,2 & 49,1 & 49,2 & 52,3 & 52,9 & 48,4 & 15,6 \\
\hline 17 & 51,7 & 48,6 & 49,4 & 51,5 & 52,2 & 48,0 & 15,7 \\
\hline 18 & 50,9 & 48,1 & 49,1 & 50,9 & 51,1 & 47,3 & 16,0 \\
\hline 19 & 49,9 & 47,5 & 48,5 & 50,5 & 49,7 & 46,5 & 16,1 \\
\hline 20 & 49,1 & 46,8 & 47,7 & 50,1 & 48,5 & 45,6 & 16,5 \\
\hline 21 & 48,9 & 46,5 & 47,3 & 49,9 & 47,7 & 45,0 & 16,8 \\
\hline 22 & 48,8 & 46,2 & 47,1 & 49,4 & 46,9 & 44,3 & 17,0 \\
\hline 23 & 47,8 & 45,3 & 46,5 & 48,2 & 45,6 & 43,2 & 17,4 \\
\hline 24 & 47,4 & 45,1 & 47,0 & 48,1 & 45,1 & 42,7 & 17,1 \\
\hline 25 & 45,6 & 43,5 & 46,6 & 47,1 & 43,6 & 41,3 & 16,2 \\
\hline 26 & 42,4 & 40,5 & 45,2 & 45,8 & 41,3 & 38,9 & 15,3 \\
\hline 27 & 39,2 & 37,1 & 43,6 & 44,8 & 38,8 & 36,5 & 15,6 \\
\hline 28 & 39,0 & 35,0 & 42,4 & 44,9 & 37,2 & 34,9 & 15,5 \\
\hline 29 & 42,9 & 34,2 & 41,5 & 45,0 & 36,2 & 34,0 & 15,6 \\
\hline 30 & 45,6 & 34,0 & 40,7 & 44,1 & 35,5 & 33,6 & 15,6 \\
\hline 31 & 44,8 & 34,3 & 40,1 & 42,7 & 35,2 & 33,6 & 15,6 \\
\hline 32 & 42,2 & 34,7 & 39,6 & 40,8 & 35,1 & 33,6 & 15,7 \\
\hline 33 & 39,5 & 35,1 & 39,1 & 38,8 & 35,1 & 33,6 & 15,6 \\
\hline 34 & 37,3 & 35,4 & 38,5 & 36,7 & 34,9 & 33,3 & 15,3 \\
\hline 35 & 38,0 & 35,6 & 37,9 & 34,8 & 34,6 & 33,0 & 15,4 \\
\hline 36 & 41,6 & 35,9 & 37,3 & 33,4 & 34,3 & 32,5 & 15,4 \\
\hline 37 & 49,2 & 45,3 & 38,2 & 35,4 & 38,9 & 42,2 & 15,4 \\
\hline 38 & 47,0 & 47,0 & 43,1 & 40,0 & 43,2 & 45,3 & 15,8 \\
\hline 39 & 43,0 & 43,7 & 43,5 & 38,0 & 41,8 & 42,7 & 15,7 \\
\hline 40 & 40,0 & 40,8 & 41,9 & 36,0 & 39,8 & 39,6 & 15,8 \\
\hline 41 & 36,9 & 37,5 & 39,5 & 33,7 & 37,3 & 36,2 & 16,0 \\
\hline 42 & 35,0 & 34,9 & 37,3 & 31,8 & 35,2 & 33,4 & 16,5 \\
\hline 43 & 30,2 & 29,3 & 35,8 & 27,9 & 34,0 & 31,8 & 17,3 \\
\hline
\end{tabular}




\begin{tabular}{|c|c|c|c|c|c|c|c|}
\hline \multirow[t]{2}{*}{ Tage } & \multicolumn{7}{|c|}{ Temperatur $\left({ }^{\circ} \mathrm{C}\right)$} \\
\hline & $\begin{array}{c}0,5 \text { g TC je } \\
\text { kg Futter } \\
\text { RM }\end{array}$ & $\begin{array}{l}\text { KT } \\
\text { RM }\end{array}$ & $\begin{array}{c}\text { 0,5 g TC je } \\
\text { kg Futter } \\
\text { SM }\end{array}$ & $\begin{array}{c}1,0 \text { g TC je } \\
\text { kg Futter } \\
\text { RM }\end{array}$ & $\begin{array}{l}\text { KT } \\
\text { SM }\end{array}$ & $\begin{array}{c}1,0 \text { g TC je } \\
\text { kg Futter } \\
\text { SM }\end{array}$ & Raumluft \\
\hline 44 & 32,7 & 29,1 & 36,8 & 30,7 & 33,6 & 31,1 & 17,5 \\
\hline 45 & 34,8 & 30,5 & 38,0 & 33,4 & 33,4 & 30,7 & 17,6 \\
\hline 46 & 34,9 & 30,9 & 37,4 & 34,8 & 32,6 & 32,0 & 17,1 \\
\hline 47 & 37,0 & 32,6 & 38,1 & 39,9 & 33,2 & 36,5 & 16,2 \\
\hline 48 & 34,3 & 30,8 & 38,4 & 37,1 & 34,3 & 35,7 & 16,0 \\
\hline 49 & 33,3 & 30,1 & 36,3 & 35,5 & 33,8 & 34,6 & 16,4 \\
\hline 50 & 32,4 & 29,8 & 34,0 & 34,4 & 32,7 & 33,6 & 16,3 \\
\hline 51 & 32,4 & 30,3 & 32,1 & 34,2 & 31,5 & 32,9 & 16,9 \\
\hline 52 & 32,5 & 30,9 & 30,8 & 34,3 & 30,9 & 32,6 & 16,8 \\
\hline 53 & 32,0 & 31,1 & 29,8 & 33,9 & 30,3 & 32,1 & 16,5 \\
\hline 54 & 30,7 & 30,5 & 28,6 & 32,6 & 29,4 & 31,0 & 16,2 \\
\hline 55 & 29,6 & 29,9 & 27,3 & 31,3 & 28,4 & 29,8 & 16,3 \\
\hline 56 & 28,9 & 29,8 & 26,3 & 30,7 & 27,6 & 29,2 & 16,3 \\
\hline 57 & 28,3 & 29,9 & 25,6 & 30,2 & 27,1 & 28,6 & 16,8 \\
\hline 58 & 28,2 & 30,8 & 25,3 & 30,1 & 26,8 & 26,9 & 17,1 \\
\hline 59 & 29,3 & 35,5 & 25,0 & 32,1 & 26,8 & 24,8 & 17,6 \\
\hline 60 & 30,1 & 35,8 & 25,5 & 32,7 & 27,5 & 25,2 & 17,4 \\
\hline 61 & 30,3 & 33,8 & 27,1 & 32,2 & 28,8 & 26,3 & 16,8 \\
\hline 62 & 28,9 & 31,3 & 28,6 & 30,6 & 29,8 & 27,2 & 16,5 \\
\hline 63 & 26,1 & 28,4 & 28,2 & 27,9 & 28,9 & 26,6 & 16,4 \\
\hline 64 & 25,7 & 28,4 & 28,1 & 27,4 & 28,4 & 26,5 & 16,9 \\
\hline 65 & 25,0 & 27,8 & 27,8 & 26,6 & 27,9 & 26,2 & 16,9 \\
\hline 66 & 24,7 & 27,4 & 27,5 & 26,2 & 27,4 & 25,9 & 17,4 \\
\hline 67 & 24,7 & 27,4 & 27,1 & 26,2 & 27,0 & 25,8 & 17,5 \\
\hline 68 & 24,5 & 27,1 & 27,0 & 26,1 & 26,9 & 25,7 & 17,7 \\
\hline 69 & 24,5 & 27,0 & 26,8 & 26,0 & 26,7 & 25,7 & 17,9 \\
\hline 70 & 24,0 & 26,3 & 26,1 & 25,4 & 26,1 & 25,2 & 18,0 \\
\hline 71 & 23,0 & 25,1 & 24,9 & 24,3 & 24,9 & 24,2 & 17,4 \\
\hline 72 & 23,6 & 25,5 & 25,6 & 25,4 & 24,8 & 24,8 & 17,6 \\
\hline 73 & 25,4 & 26,9 & 27,7 & 26,3 & 27,3 & 26,8 & 18,6 \\
\hline 74 & 25,3 & 26,5 & 28,3 & 26,1 & 27,8 & 27,3 & 18,9 \\
\hline 75 & 24,3 & 25,5 & 28,1 & 25,3 & 27,5 & 27,0 & 18,7 \\
\hline 76 & 22,9 & 24,1 & 26,7 & 23,9 & 26,1 & 25,7 & 18,4 \\
\hline 77 & 22,5 & 23,6 & 25,8 & 23,3 & 25,3 & 25,0 & 18,5 \\
\hline 78 & 22,4 & 23,5 & 25,4 & 23,2 & 25,0 & 24,8 & 18,2 \\
\hline 79 & 21,6 & 22,6 & 24,5 & 22,4 & 24,1 & 23,9 & 17,7 \\
\hline 80 & 20,4 & 21,4 & 23,2 & 21,2 & 22,8 & 22,7 & 17,1 \\
\hline 81 & 19,9 & 20,8 & 22,4 & 20,6 & 22,1 & 22,1 & 16,8 \\
\hline 82 & 19,8 & 20,7 & 22,3 & 20,6 & 22,0 & 22,0 & 17,1 \\
\hline 83 & 19,8 & 20,6 & 22,0 & 20,5 & 21,7 & 21,8 & 17,4 \\
\hline 84 & 20,9 & 21,8 & 22,8 & 21,7 & 22,7 & 22,8 & 18,8 \\
\hline 85 & 21,3 & 22,1 & 22,8 & 21,9 & 22,7 & 22,8 & 18,8 \\
\hline 86 & 22,1 & 22,9 & 23,7 & 22,8 & 23,5 & 23,6 & 19,6 \\
\hline $\begin{array}{l}\text { KT } \\
\text { RM } \\
\text { SM } \\
\text { TC }\end{array}$ & $\begin{array}{l}\text { Kontrolle } \\
\text { Rottemist } \\
\text { Stapelmist } \\
\text { Tetrazyklin }\end{array}$ & & & & & & \\
\hline
\end{tabular}


Tabelle 35: Tagesmittelwerte des Temperaturverlaufs im Masthähnchenfestmist der acht Komposter und der Raumluft, Experiment 2

\begin{tabular}{|c|c|c|c|c|c|c|c|c|c|}
\hline \multirow[t]{2}{*}{ Tage } & \multicolumn{9}{|c|}{ Temperatur $\left({ }^{\circ} \mathrm{C}\right)$} \\
\hline & $\begin{array}{l}\text { KT } \\
\text { RM }\end{array}$ & $\begin{array}{c}1 \\
\mathbf{R M}\end{array}$ & $\begin{array}{c}2 \\
\mathbf{R M}\end{array}$ & $\begin{array}{c}3 \\
\text { RM }\end{array}$ & $\begin{array}{l}\text { KT } \\
\text { SM }\end{array}$ & $\begin{array}{c}1 \\
\text { SM }\end{array}$ & $\begin{array}{c}2 \\
\mathbf{S M}\end{array}$ & $\begin{array}{c}\mathbf{3} \\
\mathbf{S M}\end{array}$ & Raumluft \\
\hline 1 & 22,5 & 22,0 & 21,2 & 23,1 & 22,5 & 23,4 & 23,6 & 21,9 & 19,0 \\
\hline 2 & 34,4 & 35,2 & 31,4 & 38,6 & 32,6 & 32,0 & 34,5 & 30,9 & 19,0 \\
\hline 3 & 47,0 & 48,6 & 46,7 & 48,8 & 45,6 & 42,2 & 46,5 & 42,4 & 19,9 \\
\hline 4 & 46,6 & 46,4 & 46,6 & 45,5 & 49,1 & 47,1 & 46,6 & 48,5 & 20,6 \\
\hline 5 & 46,0 & 45,7 & 45,1 & 45,4 & 46,2 & 45,0 & 43,1 & 45,8 & 21,2 \\
\hline 6 & 49,1 & 49,6 & 48,4 & 49,5 & 45,5 & 44,0 & 43,5 & 44,3 & 21,6 \\
\hline 7 & 50,9 & 50,4 & 49,9 & 50,0 & 48,5 & 45,8 & 46,4 & 46,0 & 22,0 \\
\hline 8 & 50,3 & 49,8 & 49,2 & 49,4 & 49,5 & 46,8 & 46,9 & 47,1 & 22,4 \\
\hline 9 & 50,1 & 49,8 & 49,4 & 49,4 & 50,3 & 47,8 & 47,1 & 48,1 & 22,7 \\
\hline 10 & 49,7 & 50,0 & 49,6 & 49,5 & 50,1 & 48,1 & 47,0 & 48,4 & 23,5 \\
\hline 11 & 49,7 & 50,2 & 50,0 & 49,6 & 50,0 & 48,5 & 47,2 & 48,7 & 24,0 \\
\hline 12 & 49,3 & 49,7 & 49,7 & 49,3 & 49,8 & 48,6 & 47,2 & 48,9 & 23,9 \\
\hline 13 & 48,9 & 49,0 & 49,2 & 48,8 & 49,0 & 48,3 & 46,3 & 48,4 & 24,4 \\
\hline 14 & 49,2 & 48,7 & 48,3 & 48,7 & 48,9 & 47,7 & 45,8 & 47,9 & 24,7 \\
\hline 15 & 49,3 & 48,7 & 48,4 & 48,7 & 49,8 & 48,6 & 46,7 & 48,7 & 25,0 \\
\hline 16 & 47,6 & 46,3 & 45,7 & 45,9 & 48,5 & 47,7 & 46,0 & 47,7 & 24,8 \\
\hline 17 & 47,5 & 45,6 & 45,0 & 45,9 & 48,3 & 47,1 & 46,1 & 47,5 & 24,1 \\
\hline 18 & 47,9 & 45,8 & 45,4 & 47,1 & 48,5 & 46,6 & 46,5 & 47,4 & 24,5 \\
\hline 19 & 49,2 & 47,6 & 47,1 & 49,3 & 49,6 & 47,3 & 47,4 & 48,2 & 24,9 \\
\hline 20 & 49,5 & 48,6 & 48,2 & 50,1 & 50,3 & 48,0 & 47,9 & 49,0 & 24,3 \\
\hline 21 & 48,6 & 47,8 & 47,7 & 49,4 & 49,9 & 47,7 & 47,5 & 48,9 & 24,0 \\
\hline 22 & 48,4 & 47,9 & 48,1 & 49,1 & 49,4 & 47,7 & 47,3 & 48,8 & 24,5 \\
\hline 23 & 48,4 & 47,7 & 48,6 & 49,1 & 47,9 & 47,0 & 47,1 & 47,6 & 24,5 \\
\hline 24 & 50,4 & 49,3 & 49,8 & 52,6 & 49,7 & 47,2 & 49,6 & 48,6 & 24,3 \\
\hline 25 & 51,0 & 50,2 & 50,2 & 54,5 & 50,0 & 47,1 & 49,9 & 48,8 & 23,6 \\
\hline 26 & 51,2 & 50,6 & 50,3 & 54,8 & 50,2 & 46,8 & 50,0 & 48,5 & 23,5 \\
\hline 27 & 51,2 & 50,7 & 50,2 & 54,5 & 50,6 & 46,6 & 50,3 & 48,4 & 23,5 \\
\hline 28 & 50,9 & 50,7 & 50,2 & 53,9 & 50,8 & 46,5 & 50,5 & 48,3 & 23,4 \\
\hline 29 & 49,9 & 49,7 & 48,3 & 51,1 & 50,9 & 46,6 & 50,5 & 48,2 & 23,8 \\
\hline 30 & 57,4 & 62,7 & 55,6 & 59,0 & 50,9 & 46,4 & 50,3 & 47,7 & 23,9 \\
\hline 31 & 55,9 & 58,8 & 53,1 & 53,7 & 51,2 & 46,6 & 50,7 & 47,8 & 23,7 \\
\hline 32 & 52,9 & 53,5 & 48,5 & 47,7 & 51,0 & 46,3 & 50,5 & 47,7 & 23,0 \\
\hline 33 & 51,6 & 50,9 & 46,3 & 45,3 & 50,4 & 45,8 & 49,9 & 47,3 & 22,7 \\
\hline 34 & 51,7 & 49,6 & 45,1 & 44,6 & 50,0 & 45,5 & 49,6 & 47,2 & 23,4 \\
\hline 35 & 50,8 & 47,3 & 43,5 & 43,3 & 50,4 & 45,8 & 50,0 & 47,6 & 23,4 \\
\hline 36 & 48,0 & 43,7 & 40,7 & 40,6 & 50,5 & 45,9 & 50,0 & 47,7 & 23,0 \\
\hline 37 & 49,1 & 51,5 & 44,5 & 42,4 & 49,3 & 45,0 & 49,0 & 46,6 & 22,5 \\
\hline 38 & 48,0 & 47,4 & 41,8 & 40,1 & 49,9 & 44,7 & 50,2 & 46,8 & 22,8 \\
\hline 39 & 47,2 & 44,3 & 40,0 & 38,6 & 50,1 & 44,9 & 50,5 & 47,1 & 23,1 \\
\hline 40 & 45,8 & 42,3 & 39,3 & 37,9 & 50,1 & 45,1 & 50,3 & 47,3 & 23,2 \\
\hline 41 & 43,4 & 40,1 & 37,8 & 36,3 & 49,8 & 45,1 & 49,9 & 47,2 & 22,9 \\
\hline 42 & 42,2 & 39,9 & 37,6 & 35,7 & 49,4 & 44,9 & 49,2 & 46,9 & 23,1 \\
\hline 43 & 43,5 & 43,3 & 41,2 & 38,0 & 47,8 & 44,2 & 47,2 & 45,6 & 22,5 \\
\hline 44 & 59,4 & 57,3 & 52,6 & 56,1 & 47,6 & 42,6 & 45,1 & 43,7 & 22,2 \\
\hline 45 & 54,3 & 48,5 & 44,6 & 47,8 & 50,6 & 43,5 & 47,6 & 45,0 & 21,9 \\
\hline 46 & 49,2 & 43,5 & 40,3 & 41,9 & 50,3 & 43,6 & 47,5 & 44,7 & 21,6 \\
\hline 47 & 46,1 & 40,5 & 37,7 & 38,7 & 49,0 & 42,9 & 46,6 & 43,6 & 21,5 \\
\hline
\end{tabular}




\begin{tabular}{lccccccccc}
\hline Tage & \multicolumn{7}{c}{$\mathbf{7}$ Temperatur $\left({ }^{\circ} \mathbf{C}\right)$} \\
& \multicolumn{1}{c}{ KT } & $\mathbf{1}$ & $\mathbf{2}$ & $\mathbf{3}$ & $\mathbf{K T}$ & $\mathbf{1}$ & $\mathbf{2}$ & $\mathbf{3}$ & Raumluft \\
& $\mathbf{R M}$ & $\mathbf{R M}$ & $\mathbf{R M}$ & $\mathbf{R M}$ & $\mathbf{S M}$ & $\mathbf{S M}$ & $\mathbf{S M}$ & $\mathbf{S M}$ & \\
\hline 48 & 44,2 & 37,2 & 35,7 & 36,4 & 48,0 & 42,1 & 45,7 & 42,5 & 21,7 \\
49 & 43,3 & 34,9 & 34,2 & 34,5 & 47,3 & 41,5 & 45,3 & 41,7 & 22,0 \\
50 & 42,5 & 33,8 & 33,3 & 33,6 & 47,0 & 41,1 & 45,2 & 41,2 & 22,2 \\
51 & 40,4 & 32,7 & 32,0 & 32,5 & 46,9 & 40,8 & 45,1 & 40,9 & 22,0 \\
52 & 38,4 & 31,7 & 31,5 & 32,0 & 46,4 & 40,4 & 44,6 & 40,3 & 22,3 \\
53 & 38,6 & 32,7 & 33,6 & 35,8 & 46,0 & 39,7 & 44,3 & 39,5 & 22,1 \\
54 & 37,1 & 32,3 & 32,8 & 35,8 & 46,2 & 39,5 & 44,8 & 39,3 & 22,0 \\
55 & 36,2 & 32,1 & 32,1 & 34,7 & 46,1 & 39,4 & 44,7 & 39,3 & 22,3 \\
56 & 36,0 & 32,3 & 32,1 & 34,2 & 46,0 & 39,5 & 44,4 & 39,3 & 22,8 \\
57 & 34,5 & 31,5 & 31,1 & 33,1 & 43,4 & 37,7 & 41,7 & 37,5 & 22,4 \\
\hline KT & Kontrolle & & & & & & & & \\
RM & Rottemist & & & & & & & &
\end{tabular}

Tabelle 36: Tetrazyklin-Gehalte im Masthähnchenfestmist der sechs Komposter über den dreimonatigen Lagerungszeitraum, Experiment 1

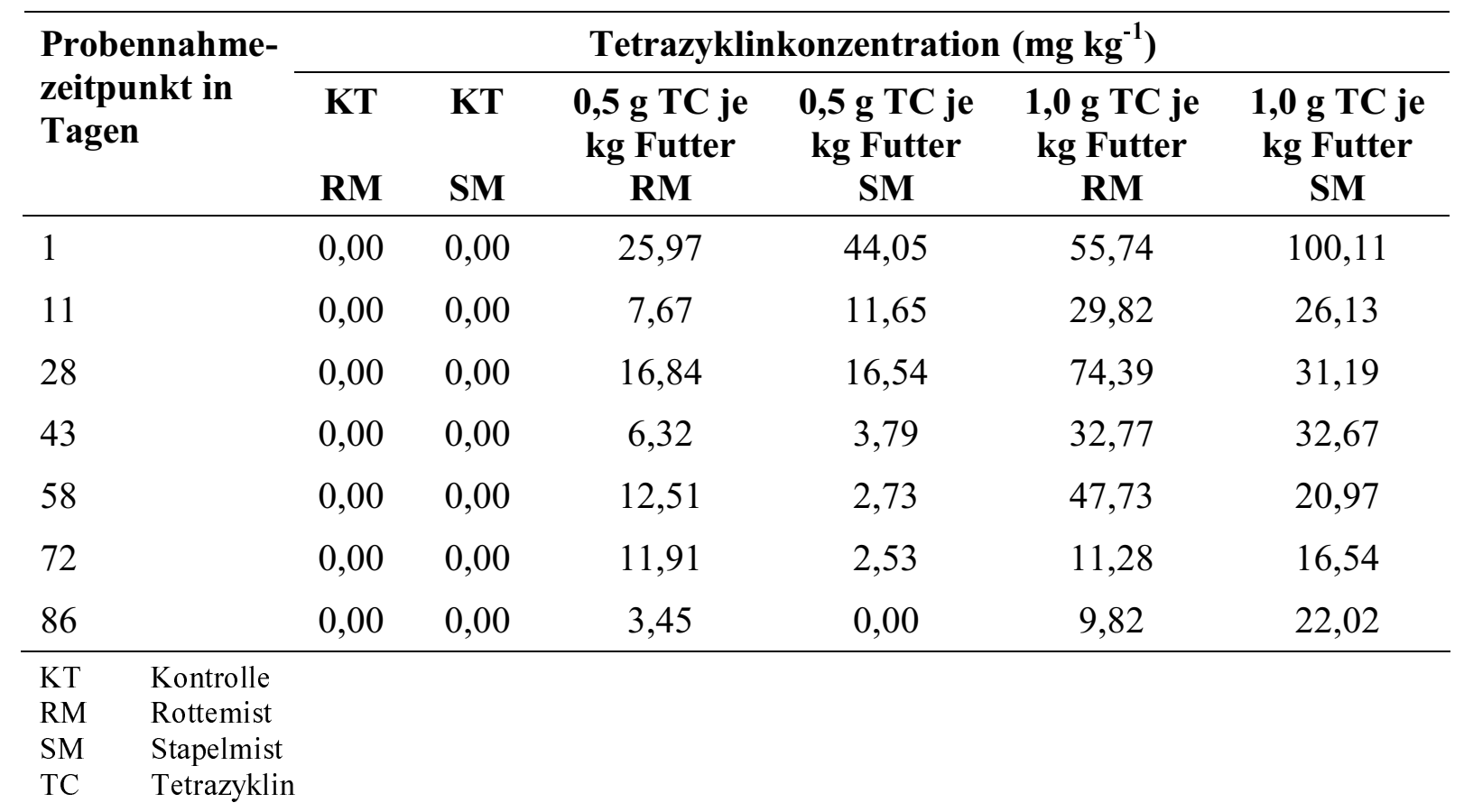


Tabelle 37: Tetrazyklin-Gehalte im Masthähnchenfestmist der acht Komposter über den zweimonatigen Lagerungszeitraum, Experiment 2

\begin{tabular}{lccccc}
\hline \multirow{2}{*}{ Komposter } & \multicolumn{5}{c}{ Tetrazyklinkonzentration $\left(\mathbf{m g ~ k g}^{\mathbf{- 1}}\right)$} \\
\cline { 2 - 6 } & Tag 1 & Tag $\mathbf{1 5}$ & Tag 29 & Tag 43 & Tag 57 \\
\hline Kontrolle RM & 5,65 & 0,00 & 0,00 & 0,00 & 0,00 \\
1 RM & 54,65 & 9,09 & 4,15 & 7,69 & 7,43 \\
2 RM & 47,34 & 11,56 & 2,27 & 7,71 & 1,66 \\
3 RM & 51,17 & 12,99 & 3,90 & 8,51 & 5,60 \\
Kontrolle SM & 5,65 & 0,00 & 0,00 & 0,00 & 0,00 \\
1 SM & 56,34 & 5,25 & 1,73 & 5,72 & 4,06 \\
2 SM & 39,29 & 4,05 & 3,74 & 3,19 & 2,20 \\
3 SM & 35,15 & 3,59 & 3,07 & 1,00 & 1,84 \\
\hline RM $\quad$ Rottemist & & & & & \\
SM Stapelmist & & &
\end{tabular}

Tabelle 38: Ermittelte Wirkstoffkonzentrationen von Tetrazyklin (TC), Oxytetrazyklin (OTC), Chlortetrazyklin (CTC), Sulfadiazin (SD) und Sulfamethazin (SM) des Screenings der Schweinegülleproben aus dem Jahr 1999/2000, n = 168 (TS = Trockensubstanz; $\mathrm{N}=$ Stickstoff)

\begin{tabular}{|c|c|c|c|c|c|c|c|}
\hline Proben-Nr. & $\begin{array}{c}\text { TS } \\
(\%)\end{array}$ & $\begin{array}{c}\mathrm{N} \\
(\%)\end{array}$ & $\begin{array}{c}\text { TC } \\
\left(\mathrm{mg} \mathrm{kg}^{-1}\right)\end{array}$ & $\begin{array}{c}\text { OTC } \\
\left(\mathrm{mg} \mathrm{kg}^{-1}\right)\end{array}$ & $\begin{array}{c}\text { CTC } \\
\left(\mathrm{mg} \mathrm{kg}^{-1}\right)\end{array}$ & $\begin{array}{c}\text { SD } \\
\left(\mathrm{mg} \mathrm{kg}^{-1}\right)\end{array}$ & $\begin{array}{c}\mathrm{SM} \\
\left(\mathrm{mg} \mathrm{kg}^{-1}\right)\end{array}$ \\
\hline 1 & 3,2 & 0,36 & $*$ & $*$ & $*$ & $*$ & 1,4 \\
\hline 2 & 8,0 & 0,41 & $*$ & $*$ & $*$ & $*$ & 2,9 \\
\hline 3 & 13,0 & 0,94 & 2,5 & * & * & 3,4 & 9,3 \\
\hline 4 & 7,7 & 0,60 & 13,4 & $*$ & 2,4 & $*$ & 3,4 \\
\hline 5 & 2,5 & 0,34 & $*$ & $*$ & $*$ & * & 2,2 \\
\hline 6 & 7,2 & 0,67 & $*$ & $*$ & 6,1 & $*$ & 1,1 \\
\hline 7 & 0,9 & 0,20 & $*$ & $*$ & $*$ & $*$ & 2,0 \\
\hline 8 & 13,3 & 0,46 & $*$ & $*$ & $*$ & $*$ & $*$ \\
\hline 9 & 12,3 & 0,76 & $*$ & $*$ & $*$ & $*$ & 9,4 \\
\hline 10 & 6,4 & 0,53 & 1,2 & $*$ & $*$ & $*$ & $*$ \\
\hline 11 & 5,2 & 0,39 & $*$ & $*$ & $*$ & $*$ & $*$ \\
\hline 12 & 2,3 & 0,24 & 18,5 & $*$ & 3,6 & $*$ & 6,5 \\
\hline 13 & 5,0 & 0,39 & $*$ & * & 8,1 & * & 4,0 \\
\hline 14 & 6,8 & 0,80 & 7,6 & $*$ & $*$ & $*$ & 20,8 \\
\hline 15 & 6,8 & 0,56 & 0,8 & $*$ & * & $*$ & $*$ \\
\hline 16 & 1,8 & 0,51 & 0,9 & $*$ & $*$ & $*$ & $*$ \\
\hline 17 & 4,2 & 0,47 & 7,6 & $*$ & $*$ & $*$ & 5,7 \\
\hline 18 & 12,2 & 0,91 & 3,3 & * & $*$ & * & 2,1 \\
\hline 19 & 7,4 & 0,61 & 3,2 & $*$ & 6,6 & $*$ & 7,7 \\
\hline 20 & 4,5 & 0,63 & * & $*$ & $*$ & $*$ & 2,9 \\
\hline 21 & 5,4 & 0,64 & $*$ & $*$ & $*$ & $*$ & $*$ \\
\hline 22 & 2,3 & 0,28 & 0,7 & $*$ & $*$ & $*$ & $*$ \\
\hline 23 & 3,0 & 0,36 & $*$ & $*$ & $*$ & $*$ & 6,2 \\
\hline
\end{tabular}




\begin{tabular}{|c|c|c|c|c|c|c|c|}
\hline Proben-Nr. & $\begin{array}{c}\text { TS } \\
(\%)\end{array}$ & $\begin{array}{c}N \\
(\%)\end{array}$ & $\begin{array}{c}\text { TC } \\
\left(\mathrm{mg} \mathrm{kg}^{-1}\right)\end{array}$ & $\begin{array}{c}\text { OTC } \\
\left(\mathrm{mg} \mathrm{kg}^{-1}\right)\end{array}$ & $\begin{array}{c}\mathrm{CTC} \\
\left(\mathrm{mg} \mathrm{kg}^{-1}\right)\end{array}$ & $\begin{array}{c}\text { SD } \\
\left(\mathrm{mg} \mathrm{kg}^{-1}\right)\end{array}$ & $\begin{array}{c}\mathrm{SM} \\
\left(\mathrm{mg} \mathrm{kg}^{-1}\right)\end{array}$ \\
\hline 24 & 8,7 & 0,75 & $*$ & $*$ & $*$ & $*$ & 3,6 \\
\hline 25 & 2,2 & 0,35 & $*$ & $*$ & $*$ & $*$ & 1,9 \\
\hline 26 & 1,8 & 0,25 & $*$ & $*$ & 3,6 & $*$ & 2,2 \\
\hline 27 & 3,8 & 0,37 & $*$ & $*$ & $*$ & $*$ & $*$ \\
\hline 28 & 9,7 & 0,65 & $*$ & $*$ & $*$ & $*$ & $*$ \\
\hline 29 & 3,5 & 0,29 & 10,5 & $*$ & $*$ & $*$ & 16,6 \\
\hline 30 & 3,3 & 0,30 & $*$ & $*$ & * & $*$ & $*$ \\
\hline 31 & 6,4 & 0,57 & 9,2 & $*$ & $*$ & $*$ & 7,0 \\
\hline 32 & 2,0 & 0,25 & $*$ & $*$ & $*$ & $*$ & $*$ \\
\hline 33 & 5,5 & 0,64 & $*$ & $*$ & $*$ & $*$ & 4,9 \\
\hline 34 & 5,0 & 0,67 & 2,8 & $*$ & 3,6 & $*$ & 4,4 \\
\hline 35 & 9,0 & 0,76 & 4,5 & $*$ & $*$ & $*$ & 11,2 \\
\hline 36 & 3,3 & 0,50 & $*$ & $*$ & * & * & $*$ \\
\hline 37 & 11,5 & 0,69 & 3,1 & $*$ & $*$ & $*$ & 9,3 \\
\hline 38 & 7,4 & 0,71 & $*$ & $*$ & $*$ & $*$ & 3,2 \\
\hline 39 & 12,6 & 0,98 & $*$ & $*$ & 3,2 & $*$ & $*$ \\
\hline 40 & 8,6 & 0,82 & 15,1 & $*$ & $*$ & $*$ & 18,0 \\
\hline 41 & 3,6 & 0,41 & $*$ & $*$ & $*$ & $*$ & $*$ \\
\hline 42 & 6,1 & 0,29 & $*$ & $*$ & $*$ & $*$ & 1,9 \\
\hline 43 & 14,7 & 0,98 & 29,2 & $*$ & $*$ & 2,1 & 12,2 \\
\hline 44 & 9,0 & 0,80 & $*$ & $*$ & * & $*$ & 4,4 \\
\hline 45 & 9,7 & 0,86 & $*$ & $*$ & $*$ & $*$ & 4,2 \\
\hline 46 & 8,4 & 0,81 & 3,7 & $*$ & $*$ & $*$ & 2,9 \\
\hline 47 & 6,0 & 0,46 & 1,0 & $*$ & $*$ & $*$ & 2,7 \\
\hline 48 & 4,9 & 0,38 & $*$ & $*$ & $*$ & $*$ & $*$ \\
\hline 49 & 7,4 & 1,01 & 2,9 & $*$ & $*$ & $*$ & 2,8 \\
\hline 50 & 5,8 & 0,50 & $*$ & $*$ & $*$ & $*$ & $*$ \\
\hline 51 & 5,7 & 0,62 & 22,5 & $*$ & $*$ & $*$ & 5,8 \\
\hline 52 & 3,0 & 0,30 & $*$ & $*$ & $*$ & $*$ & $*$ \\
\hline 53 & 1,9 & 0,25 & $*$ & $*$ & $*$ & $*$ & $*$ \\
\hline 54 & 6,7 & 0,43 & * & $*$ & $*$ & $*$ & $*$ \\
\hline 55 & 1,8 & 0,20 & $*$ & $*$ & * & * & $*$ \\
\hline 56 & 2,3 & 0,32 & $*$ & $*$ & $*$ & $*$ & 3,4 \\
\hline 57 & 1,8 & 0,24 & $*$ & $*$ & $*$ & $*$ & $*$ \\
\hline 58 & 2,7 & 0,48 & 1,1 & $*$ & 8,2 & $*$ & 6,8 \\
\hline 59 & 3,0 & 0,32 & $*$ & $*$ & $*$ & $*$ & 1,7 \\
\hline 60 & 7,5 & 0,55 & 2,4 & $*$ & $*$ & $*$ & 4,3 \\
\hline 61 & 1,8 & 0,41 & $*$ & $*$ & $*$ & $*$ & $*$ \\
\hline 62 & 1,7 & 0,36 & 7,6 & $*$ & $*$ & $*$ & 1,9 \\
\hline 63 & 1,1 & 0,24 & $*$ & $*$ & $*$ & $*$ & $*$ \\
\hline 64 & 4,5 & 0,65 & 17,6 & $*$ & $*$ & $*$ & 15,7 \\
\hline 65 & 1,9 & 0,42 & $*$ & $*$ & $*$ & $*$ & 4,7 \\
\hline 66 & 3,6 & 0,44 & $*$ & $*$ & $*$ & $*$ & $*$ \\
\hline 67 & 7,0 & 0,50 & $*$ & $*$ & $*$ & $*$ & 2,1 \\
\hline 68 & 5,2 & 0,46 & 4,2 & $*$ & 3,4 & $*$ & 5,4 \\
\hline 69 & 1,0 & 0,19 & $*$ & $*$ & $*$ & $*$ & $*$ \\
\hline 70 & 4,5 & 0,50 & $*$ & $*$ & $*$ & 29,3 & $*$ \\
\hline 71 & 6,9 & 0,35 & $*$ & $*$ & $*$ & $*$ & 3,4 \\
\hline 72 & 2,2 & 0,28 & $*$ & $*$ & $*$ & $*$ & 1,6 \\
\hline 73 & 4,8 & 0,58 & 9,6 & $*$ & $*$ & * & 17,1 \\
\hline
\end{tabular}




\begin{tabular}{|c|c|c|c|c|c|c|c|}
\hline Proben-Nr. & $\begin{array}{c}\text { TS } \\
(\%)\end{array}$ & $\begin{array}{c}N \\
(\%)\end{array}$ & $\begin{array}{c}\mathrm{TC} \\
\left(\mathrm{mg} \mathrm{kg}^{-1}\right)\end{array}$ & $\underset{\left(\mathrm{mg} \mathrm{kg}^{-1}\right)}{\text { OTC }}$ & $\underset{\left(\mathrm{mg} \mathrm{kg}^{-1}\right)}{\mathrm{CTC}}$ & $\begin{array}{c}\text { SD } \\
\left(\mathrm{mg} \mathrm{kg}^{-1}\right)\end{array}$ & $\begin{array}{c}\mathrm{SM} \\
\left(\mathrm{mg} \mathrm{kg}^{-1}\right)\end{array}$ \\
\hline 74 & 5,8 & 0,67 & 3,6 & $*$ & 4,8 & $*$ & 7,5 \\
\hline 75 & 2,4 & 0,29 & 3,9 & $*$ & $*$ & $*$ & 3,7 \\
\hline 76 & 9,3 & 0,84 & $*$ & $*$ & * & * & 3,0 \\
\hline 77 & 2,2 & 0,24 & * & * & * & * & $*$ \\
\hline 78 & 5,2 & 0,59 & 13,9 & $*$ & 8,8 & $*$ & 27,2 \\
\hline 79 & 10,9 & 0,87 & 7,1 & $*$ & 4,5 & 235,1 & 18,6 \\
\hline 80 & 2,7 & 0,24 & $*$ & * & * & * & $*$ \\
\hline 81 & 3,1 & 0,32 & $*$ & $*$ & * & * & 1,4 \\
\hline 82 & 1,6 & 0,25 & $*$ & $*$ & * & * & 6,9 \\
\hline 83 & 1,5 & 0,32 & $*$ & $*$ & $*$ & * & $*$ \\
\hline 84 & 2,2 & 0,34 & $*$ & $*$ & 5,3 & * & * \\
\hline 85 & 2,7 & 0,35 & 7,2 & * & 2,2 & * & 19,4 \\
\hline 86 & 1,6 & 0,20 & $*$ & $*$ & $*$ & * & $*$ \\
\hline 87 & 4,3 & 0,53 & $*$ & * & * & * & * \\
\hline 88 & 1,4 & 0,21 & $*$ & $*$ & * & * & * \\
\hline 89 & 10,8 & 0,48 & $*$ & $*$ & $*$ & 9,3 & $*$ \\
\hline 90 & k.A. & k.A. & * & * & * & * & * \\
\hline 91 & 2,1 & 0,50 & $*$ & $*$ & 3,5 & * & $*$ \\
\hline 92 & 10,7 & 0,72 & 2,8 & $*$ & $*$ & * & 6,7 \\
\hline 93 & k.A. & 0,65 & 12,6 & $*$ & * & * & 20,1 \\
\hline 94 & 8,8 & 0,64 & 13,5 & $*$ & $*$ & $*$ & 3,2 \\
\hline 95 & 5,4 & 0,67 & 0,7 & * & * & 2,1 & 4,8 \\
\hline 96 & 0,5 & 0,10 & $*$ & * & * & $*$ & $*$ \\
\hline 97 & 2,9 & 0,36 & 1,6 & $*$ & $*$ & $*$ & $*$ \\
\hline 98 & 1,6 & 0,29 & 1,1 & $*$ & $*$ & 2,1 & 9,6 \\
\hline 99 & 7,5 & 0,73 & 24,0 & $*$ & * & $*$ & 62,1 \\
\hline 100 & k.A. & k.A. & * & $*$ & $*$ & 2,1 & $*$ \\
\hline 101 & 1,8 & 0,27 & $*$ & $*$ & $*$ & 1,0 & $*$ \\
\hline 102 & 6,4 & 0,53 & $*$ & $*$ & $*$ & $*$ & $*$ \\
\hline 103 & 1,7 & 0,32 & $*$ & $*$ & * & * & * \\
\hline 104 & 4,0 & 0,42 & $*$ & $*$ & $*$ & $*$ & $*$ \\
\hline 105 & 3,8 & 0,40 & $*$ & $*$ & $*$ & $*$ & 2,0 \\
\hline 106 & 2,7 & 0,53 & $*$ & $*$ & $*$ & * & $*$ \\
\hline 107 & 1,2 & 0,17 & $*$ & $*$ & $*$ & $*$ & $*$ \\
\hline 108 & 3,8 & 0,34 & $*$ & * & * & * & * \\
\hline 109 & 4,1 & 0,41 & 2,6 & $*$ & $*$ & $*$ & 4,1 \\
\hline 110 & 5,2 & 0,53 & $*$ & * & $*$ & $*$ & $*$ \\
\hline 111 & 11,7 & 0,93 & 7,0 & $*$ & * & * & 5,1 \\
\hline 112 & 2,4 & 0,44 & $*$ & $*$ & $*$ & $*$ & $*$ \\
\hline 113 & 3,3 & 0,38 & 0,6 & $*$ & $*$ & * & 1,9 \\
\hline 114 & 3,8 & 0,37 & $*$ & $*$ & $*$ & $*$ & $*$ \\
\hline 115 & 1,5 & 0,31 & $*$ & $*$ & $*$ & $*$ & $*$ \\
\hline 116 & 3,3 & 0,36 & $*$ & * & * & * & $*$ \\
\hline 117 & 2,6 & 0,31 & 5,5 & $*$ & $*$ & $*$ & 6,2 \\
\hline 118 & 6,2 & 0,34 & $*$ & $*$ & * & * & $*$ \\
\hline 119 & 2,8 & 0,28 & $*$ & $*$ & $*$ & $*$ & $*$ \\
\hline 120 & 2,1 & 0,35 & $*$ & $*$ & $*$ & $*$ & $*$ \\
\hline 121 & 3,5 & 0,45 & 5,7 & * & * & * & 4,0 \\
\hline 122 & 10,2 & 0,85 & $*$ & $*$ & 7,5 & * & 2,0 \\
\hline 123 & 3,3 & 0,33 & $*$ & $*$ & $*$ & $*$ & 0,7 \\
\hline
\end{tabular}




\begin{tabular}{|c|c|c|c|c|c|c|c|}
\hline Proben-Nr. & $\begin{array}{c}\text { TS } \\
(\%)\end{array}$ & $\begin{array}{c}N \\
(\%)\end{array}$ & $\begin{array}{c}\text { TC } \\
\left(\mathrm{mg} \mathrm{kg}^{-1}\right)\end{array}$ & $\begin{array}{c}\text { OTC } \\
\left(\mathrm{mg} \mathrm{kg}^{-1}\right)\end{array}$ & $\begin{array}{c}\text { CTC } \\
\left(\mathrm{mg} \mathrm{kg}^{-1}\right)\end{array}$ & $\begin{array}{c}\text { SD } \\
\left(\mathrm{mg} \mathrm{kg}^{-1}\right)\end{array}$ & $\begin{array}{c}\mathrm{SM} \\
\left(\mathrm{mg} \mathrm{kg}^{-1}\right)\end{array}$ \\
\hline 124 & 3,0 & 0,34 & $*$ & $*$ & $*$ & $*$ & 3,1 \\
\hline 125 & 3,1 & 0,30 & $*$ & * & * & $*$ & * \\
\hline 126 & 1.8 & 0.25 & $*$ & $*$ & $*$ & $*$ & $*$ \\
\hline 127 & 8,4 & 0,44 & $*$ & $*$ & * & 4,0 & * \\
\hline 128 & 6,0 & 0,67 & 5,3 & * & 330,7 & $*$ & 8,8 \\
\hline 129 & 9,1 & 0,60 & $*$ & $*$ & $*$ & $*$ & 3,9 \\
\hline 130 & 5,1 & 0,54 & $*$ & * & $*$ & $*$ & 2,4 \\
\hline 131 & 3,0 & 0,41 & * & * & 3,8 & 2,1 & $*$ \\
\hline 132 & 4,7 & 0,38 & $*$ & $*$ & $*$ & $*$ & 1,3 \\
\hline 133 & 3,8 & 0,45 & * & * & * & * & 3,2 \\
\hline 134 & 4,3 & 0,49 & * & $*$ & * & * & 2,8 \\
\hline 135 & 1,0 & 0,25 & * & * & $*$ & 2,5 & $*$ \\
\hline 136 & 1,4 & 0,26 & * & * & * & * & * \\
\hline 137 & 3,1 & 0,28 & $*$ & * & * & * & 2,3 \\
\hline 138 & 1,6 & 0,27 & 4,4 & * & * & * & 14,0 \\
\hline 139 & 3,5 & 0,55 & $*$ & * & * & * & $*$ \\
\hline 140 & 4,2 & 0,39 & 13,5 & * & $*$ & $*$ & 3,6 \\
\hline 141 & 11,9 & 0,88 & $*$ & * & * & * & $*$ \\
\hline 142 & 0,9 & 0,15 & 4,1 & * & * & * & * \\
\hline 143 & 3,7 & 0,41 & 6,4 & * & * & * & * \\
\hline 144 & 3,1 & 0,36 & 0,7 & * & * & * & 2,2 \\
\hline 145 & 2,9 & 0,40 & $*$ & * & * & * & $*$ \\
\hline 146 & 3,4 & 0,49 & * & * & * & * & 4,3 \\
\hline 147 & 1,8 & 0,41 & * & * & 8,2 & * & 3,8 \\
\hline 148 & 4,7 & 0,41 & 38,3 & $*$ & 3,4 & $*$ & 13,1 \\
\hline 149 & 10,3 & 0,61 & 33,1 & * & * & 9,1 & 33,6 \\
\hline 150 & 7,4 & 0,82 & 9,8 & * & $*$ & * & 20,4 \\
\hline 151 & 4,7 & 0,59 & 25,7 & * & * & * & 11,1 \\
\hline 152 & 5,8 & 0,73 & 2,1 & * & * & 4,9 & 6,0 \\
\hline 153 & 6,4 & 0,52 & * & $*$ & * & $*$ & $*$ \\
\hline 154 & 0,8 & 0,18 & * & * & * & * & * \\
\hline 155 & 3,8 & 0,39 & $*$ & $*$ & $*$ & $*$ & $*$ \\
\hline 156 & 2,9 & 0,12 & $*$ & * & * & * & 6,0 \\
\hline 157 & 7,3 & 0,43 & 10,8 & * & 2,3 & * & 7,9 \\
\hline 158 & 6,4 & 0,74 & $*$ & * & $*$ & $*$ & $*$ \\
\hline 159 & 5,2 & 0,50 & 3,4 & $*$ & * & * & 6,3 \\
\hline 160 & 2,7 & 0,36 & 44,4 & 4,8 & 29,9 & $*$ & 17,8 \\
\hline 161 & 10,5 & 0,64 & 1,7 & $*$ & 11,3 & * & $*$ \\
\hline 162 & 3,1 & 0,46 & 0,8 & $*$ & 4,1 & * & 3,2 \\
\hline 163 & 5,1 & 0,57 & 6,5 & $*$ & $*$ & $*$ & 1,8 \\
\hline 164 & 3,5 & 0,31 & $*$ & * & * & * & $*$ \\
\hline 165 & 1,9 & 0,32 & $*$ & $*$ & $*$ & * & $*$ \\
\hline 166 & 2,5 & 0,29 & 16,3 & * & 4,3 & $*$ & 39,1 \\
\hline 167 & 6,5 & 0,74 & 11,2 & $*$ & $*$ & * & 6,8 \\
\hline 168 & 8,3 & 0,79 & 8,6 & $*$ & $*$ & * & 4,1 \\
\hline
\end{tabular}

* nicht nachweisbar; unterhalb der Bestimmungsgrenzen von $0,8 \mathrm{mg} \mathrm{kg}^{-1}$ für TC, CTC, SD, SM und unterhalb $1,0 \mathrm{mg} \mathrm{kg}^{-1}$ für OTC

k.A. $=$ keine Angabe 
Tabelle 39: Ermittelte Wirkstoffkonzentrationen von Tetrazyklin (TC), Oxytetrazyklin (OTC), Chlortetrazyklin (CTC), Sulfadiazin (SD) und Sulfamethazin (SM) des Screenings der Schweinegülleproben aus dem Jahr 2001/2002, n = 176 (TS = Trockensubstanz; $\mathrm{N}=$ Stickstoff)

\begin{tabular}{|c|c|c|c|c|c|c|c|}
\hline Proben-Nr. & $\begin{array}{c}\text { TS } \\
(\%) \\
\end{array}$ & $\begin{array}{c}\mathrm{N} \\
(\%)\end{array}$ & $\begin{array}{c}\mathrm{TC} \\
\left(\mathrm{mg} \mathrm{kg}^{-1}\right) \\
\end{array}$ & $\begin{array}{c}\text { OTC } \\
\left(\mathrm{mg} \mathrm{kg}^{-1}\right)\end{array}$ & $\begin{array}{c}\mathrm{CTC} \\
\left(\mathrm{mg} \mathrm{kg}^{-1}\right)\end{array}$ & $\begin{array}{c}\text { SD } \\
\left(\mathrm{mg} \mathrm{kg}^{-1}\right) \\
\end{array}$ & $\begin{array}{c}\mathrm{SM} \\
\left(\mathrm{mg} \mathrm{kg}^{-1}\right) \\
\end{array}$ \\
\hline 1 & k.A. & 0,19 & * & $*$ & $*$ & $*$ & 9,4 \\
\hline 2 & k.A. & 0,64 & $*$ & $*$ & $*$ & $*$ & 4,6 \\
\hline 3 & 2,7 & 0,37 & 1,2 & $*$ & $*$ & $*$ & $*$ \\
\hline 4 & 1,8 & 0,25 & $*$ & $*$ & * & * & * \\
\hline 5 & 2,6 & 0,27 & $*$ & $*$ & * & * & * \\
\hline 6 & 1,0 & 0,18 & * & $*$ & * & 1,5 & * \\
\hline 7 & 2,0 & 0,21 & $*$ & $*$ & $*$ & 4,5 & $*$ \\
\hline 8 & 2,7 & 0,28 & 8,6 & $*$ & $*$ & 17,0 & $*$ \\
\hline 9 & 2,0 & 0,22 & 0,9 & $*$ & $*$ & $*$ & $*$ \\
\hline 10 & 1,2 & 0,21 & $*$ & $*$ & $*$ & 2,0 & $*$ \\
\hline 11 & 2,0 & 0,46 & $*$ & * & * & 2,7 & 8,7 \\
\hline 12 & 2,4 & 0,37 & $*$ & $*$ & * & 3,5 & $*$ \\
\hline 13 & 1,3 & 0,36 & $*$ & $*$ & $*$ & 2,6 & $*$ \\
\hline 14 & 2,7 & 0,53 & $*$ & $*$ & * & $*$ & * \\
\hline 15 & 2,8 & 0,29 & $*$ & $*$ & * & $*$ & * \\
\hline 16 & 2,6 & 0,46 & $*$ & * & * & 2,8 & $*$ \\
\hline 17 & 2,6 & 0,25 & $*$ & $*$ & $*$ & 3,3 & $*$ \\
\hline 18 & 1,0 & 0,23 & $*$ & $*$ & $*$ & 2,5 & $*$ \\
\hline 19 & 1,9 & 0,41 & 2,2 & $*$ & * & 3,8 & 3,0 \\
\hline 20 & 2,4 & 0,35 & $*$ & $*$ & $*$ & $*$ & $*$ \\
\hline 21 & 2,7 & 0,31 & $*$ & $*$ & 1,6 & $*$ & $*$ \\
\hline 22 & 2,3 & 0,27 & $*$ & $*$ & $*$ & 2,4 & 13,0 \\
\hline 23 & 1,5 & 0,35 & $*$ & $*$ & $*$ & $*$ & $*$ \\
\hline 24 & 1,7 & 0,21 & $*$ & $*$ & * & 3,3 & * \\
\hline 25 & 0,9 & 0,23 & $*$ & $*$ & $*$ & $*$ & $*$ \\
\hline 26 & 2,1 & 0,24 & $*$ & $*$ & $*$ & 2,2 & 1,7 \\
\hline 27 & 2,8 & 0,42 & 4,0 & $*$ & $*$ & $*$ & $*$ \\
\hline 28 & 2,3 & 0,31 & 5,8 & $*$ & $*$ & $*$ & 5,8 \\
\hline 29 & 2,4 & 0,26 & 3,6 & $*$ & $*$ & $*$ & $*$ \\
\hline 30 & 2,1 & 0,24 & $*$ & $*$ & $*$ & 1,7 & 2,3 \\
\hline 31 & 1,7 & 0,33 & 4,1 & $*$ & $*$ & 7,2 & 3,3 \\
\hline 32 & 2,3 & 0,35 & $*$ & $*$ & $*$ & 1,4 & 7,2 \\
\hline 33 & 2,5 & 0,29 & $*$ & $*$ & $*$ & 3,6 & $*$ \\
\hline 34 & 2,5 & 0,40 & $*$ & $*$ & * & $*$ & 3,0 \\
\hline 35 & 2,6 & 0,23 & $*$ & $*$ & $*$ & $*$ & 1,8 \\
\hline 36 & 2,8 & 0,54 & 3,9 & $*$ & * & $*$ & 17,8 \\
\hline 37 & 2,8 & 0,38 & $*$ & $*$ & * & * & $*$ \\
\hline 38 & 1,0 & 0,17 & $*$ & $*$ & * & * & $*$ \\
\hline 39 & 0,8 & 0,29 & $*$ & $*$ & $*$ & $*$ & 2,0 \\
\hline 40 & 0,9 & 0,15 & $*$ & $*$ & $*$ & 2,4 & $*$ \\
\hline 41 & 2,5 & 0,29 & $*$ & $*$ & $*$ & $*$ & 3,4 \\
\hline 42 & 0,7 & 0,18 & $*$ & $*$ & $*$ & $*$ & 2,5 \\
\hline 43 & 2,0 & 0,32 & $*$ & $*$ & $*$ & * & $*$ \\
\hline 44 & 1,7 & 0,29 & $*$ & $*$ & 2,0 & $*$ & 2,4 \\
\hline 45 & 2,2 & 0,28 & 1,2 & $*$ & $*$ & $*$ & $*$ \\
\hline 46 & 2,8 & 0,32 & $*$ & $*$ & $*$ & $*$ & $*$ \\
\hline
\end{tabular}




\begin{tabular}{|c|c|c|c|c|c|c|c|}
\hline Proben-Nr. & $\begin{array}{c}\text { TS } \\
(\%)\end{array}$ & $\begin{array}{c} \\
(\%)\end{array}$ & $\begin{array}{c}\text { TC } \\
\left(\mathrm{mg} \mathrm{kg}^{-1}\right)\end{array}$ & $\begin{array}{c}\text { OTC } \\
\left(\mathrm{mg} \mathrm{kg}^{-1}\right)\end{array}$ & $\begin{array}{c}\text { CTC } \\
\left(\mathrm{mg} \mathrm{kg}^{-1}\right)\end{array}$ & $\begin{array}{c}\text { SD } \\
\left(\mathrm{mg} \mathrm{kg}^{-1}\right)\end{array}$ & $\begin{array}{c}\mathrm{SM} \\
\left(\mathrm{mg} \mathrm{kg}^{-1}\right)\end{array}$ \\
\hline 47 & 1,3 & 0,21 & $*$ & $*$ & $*$ & 4,2 & $*$ \\
\hline 48 & 2,4 & 0,32 & $*$ & $*$ & * & $*$ & * \\
\hline 49 & 1,7 & 0,22 & 8,1 & * & * & * & * \\
\hline 50 & 2,3 & 0,31 & 3,8 & $*$ & $*$ & * & * \\
\hline 51 & 2,7 & 0,35 & $*$ & $*$ & 1,1 & * & * \\
\hline 52 & 1,3 & 0,17 & 3,6 & $*$ & $*$ & * & * \\
\hline 53 & 5,7 & 0,63 & $*$ & $*$ & $*$ & 6,9 & $*$ \\
\hline 54 & 3,6 & 0,33 & $*$ & * & * & 3,3 & * \\
\hline 55 & 4,7 & 0,31 & $*$ & $*$ & * & 3,7 & * \\
\hline 56 & 3,7 & 0,35 & $*$ & $*$ & * & 3,3 & * \\
\hline 57 & 3,8 & 0,33 & $*$ & $*$ & * & 0,7 & * \\
\hline 58 & 3,9 & 0,51 & $*$ & 3,9 & * & $*$ & * \\
\hline 59 & 3,0 & 0,19 & $*$ & $*$ & * & * & * \\
\hline 60 & 4,7 & 0,52 & 3,3 & $*$ & * & 6,8 & 5,7 \\
\hline 61 & 3,4 & 0,48 & 8,1 & * & * & $*$ & $*$ \\
\hline 62 & 4,2 & 0,88 & $*$ & $*$ & $*$ & * & $*$ \\
\hline 63 & 3,5 & 0,39 & 2,5 & $*$ & $*$ & $*$ & $*$ \\
\hline 64 & 3,7 & 0,38 & 2,6 & * & * & * & $*$ \\
\hline 65 & 4,9 & 0,42 & 4,8 & $*$ & * & * & 3,2 \\
\hline 66 & 3,5 & 0,40 & 21,7 & $*$ & * & * & $*$ \\
\hline 67 & 5,3 & 0,39 & $*$ & $*$ & $*$ & 3,5 & 2,0 \\
\hline 68 & 5,4 & 0,47 & $*$ & $*$ & $*$ & 3,3 & $*$ \\
\hline 69 & 5,3 & 0,47 & * & * & * & $*$ & $*$ \\
\hline 70 & 3,9 & 0,51 & 12,6 & $*$ & $*$ & 4,1 & 16,0 \\
\hline 71 & 5,2 & 0,34 & $*$ & $*$ & $*$ & 3,1 & $*$ \\
\hline 72 & 6,3 & 0,54 & 6,8 & * & * & $*$ & 2,3 \\
\hline 73 & 4,0 & 0,64 & 1,1 & $*$ & * & $*$ & $*$ \\
\hline 74 & 4,1 & 0,47 & $*$ & $*$ & * & 1,9 & * \\
\hline 75 & 5,4 & 0,50 & $*$ & * & * & $*$ & * \\
\hline 76 & 5,5 & 0,48 & $*$ & $*$ & $*$ & $*$ & $*$ \\
\hline 77 & 4,3 & 0,60 & 16,2 & $*$ & $*$ & 2,2 & 12,2 \\
\hline 78 & 3,8 & 0,58 & 22,6 & $*$ & $*$ & 2,9 & $*$ \\
\hline 79 & 5,3 & 0,42 & 7,2 & * & * & $*$ & $*$ \\
\hline 80 & 4,0 & 0,43 & 9,4 & $*$ & * & 3,1 & 2,1 \\
\hline 81 & 4,9 & 0,50 & 15,8 & $*$ & $*$ & $*$ & $*$ \\
\hline 82 & 5,0 & 0,49 & 4,6 & * & 5,1 & 3,7 & $*$ \\
\hline 83 & 5,7 & 0,75 & $*$ & $*$ & $*$ & 10,1 & 6,7 \\
\hline 84 & 3,6 & 0,31 & $*$ & $*$ & $*$ & $*$ & 32,7 \\
\hline 85 & 3,7 & 0,43 & * & $*$ & * & * & 2,5 \\
\hline 86 & 3,3 & 0,49 & $*$ & $*$ & $*$ & 2,6 & 2,6 \\
\hline 87 & 4,5 & 0,64 & $*$ & * & $*$ & 3,3 & 5,6 \\
\hline 88 & 3,1 & 0,39 & $*$ & $*$ & $*$ & $*$ & $*$ \\
\hline 89 & 5,0 & 0,77 & 3,3 & $*$ & $*$ & 4,9 & 4,5 \\
\hline 90 & 3,9 & 0,53 & 6,6 & $*$ & * & $*$ & 7,1 \\
\hline 91 & 4,5 & 0,56 & 11,5 & $*$ & $*$ & 4,6 & 2,2 \\
\hline 92 & 5,3 & 0,54 & 6,7 & * & * & 3,8 & 7,2 \\
\hline 93 & 3,8 & 0,46 & 7,3 & $*$ & $*$ & 3,1 & 4,0 \\
\hline 94 & 5,1 & 0,57 & 5,5 & $*$ & $*$ & $*$ & 3,7 \\
\hline 95 & 4,1 & 0,60 & 3,5 & $*$ & * & $*$ & 7,4 \\
\hline 96 & 5,8 & 0,42 & 12,7 & * & $*$ & $*$ & 2,7 \\
\hline
\end{tabular}




\begin{tabular}{|c|c|c|c|c|c|c|c|}
\hline Proben-Nr. & $\begin{array}{c}\text { TS } \\
(\%) \\
\end{array}$ & $\begin{array}{c}N \\
(\%) \\
\end{array}$ & $\begin{array}{c}\mathrm{TC} \\
\left(\mathrm{mg} \mathrm{kg}^{-1}\right) \\
\end{array}$ & $\begin{array}{c}\text { OTC } \\
\left(\mathrm{mg} \mathrm{kg}^{-1}\right)\end{array}$ & $\begin{array}{c}\mathrm{CTC} \\
\left(\mathrm{mg} \mathrm{kg}^{-1}\right)\end{array}$ & $\begin{array}{c}\text { SD } \\
\left(\mathrm{mg} \mathrm{kg}^{-1}\right) \\
\end{array}$ & $\begin{array}{c}\mathrm{SM} \\
\left(\mathrm{mg} \mathrm{kg}^{-1}\right) \\
\end{array}$ \\
\hline 97 & 4,3 & 0,43 & 4,1 & $*$ & * & * & * \\
\hline 98 & 5,2 & 0,54 & $*$ & $*$ & $*$ & $*$ & 2,6 \\
\hline 99 & 4,0 & 0,62 & 5,8 & $*$ & $*$ & 2,8 & 1,6 \\
\hline 100 & 3,8 & 0,39 & $*$ & 25,7 & $*$ & $*$ & * \\
\hline 101 & 3,3 & 0,32 & $*$ & $*$ & 3,8 & $*$ & $*$ \\
\hline 102 & 5,2 & 0,50 & 16,9 & $*$ & * & 25,3 & 2,3 \\
\hline 103 & 3,3 & 0,35 & 6,4 & $*$ & $*$ & 10,4 & $*$ \\
\hline 104 & 3,2 & 0,39 & 22,1 & $*$ & 5,7 & $*$ & 5,6 \\
\hline 105 & 3,0 & 0,23 & $*$ & $*$ & $*$ & 2,4 & 2,0 \\
\hline 106 & 5,9 & 0,58 & 19,7 & $*$ & 6,9 & 25,0 & 4,5 \\
\hline 107 & 3,7 & 0,33 & 7,2 & * & * & 1,9 & * \\
\hline 108 & 3,7 & 0,39 & $*$ & $*$ & $*$ & 11,4 & 1,8 \\
\hline 109 & 4,6 & 0,33 & $*$ & $*$ & 2,3 & $*$ & $*$ \\
\hline 110 & 5,5 & 0,76 & $*$ & $*$ & * & 4,2 & 3,0 \\
\hline 111 & 4,7 & 0,59 & 37,6 & $*$ & * & $*$ & 10,9 \\
\hline 112 & 3,2 & 0,31 & 4,2 & $*$ & * & * & * \\
\hline 113 & 4,8 & 0,52 & 14,5 & $*$ & $*$ & $*$ & $*$ \\
\hline 114 & 4,2 & 0,52 & 14,7 & $*$ & $*$ & $*$ & 13,0 \\
\hline 115 & 4,2 & 0,46 & $*$ & * & * & 8,5 & 2,8 \\
\hline 116 & 4,1 & 0,42 & 6,9 & $*$ & $*$ & $*$ & $*$ \\
\hline 117 & 3,2 & 0,69 & $*$ & $*$ & * & * & 2,0 \\
\hline 118 & 4,3 & 0,42 & $*$ & $*$ & $*$ & * & $*$ \\
\hline 119 & 4,0 & 0,54 & 2,7 & $*$ & 5,8 & $*$ & 4,9 \\
\hline 120 & 5,9 & 0,59 & 2,0 & $*$ & * & $*$ & * \\
\hline 121 & 7,1 & 0,46 & $*$ & $*$ & $*$ & 2,2 & * \\
\hline 122 & 6,9 & 0,68 & 21,3 & $*$ & $*$ & 5,8 & 2,9 \\
\hline 123 & 7,1 & 0,59 & $*$ & $*$ & $*$ & 1,9 & $*$ \\
\hline 124 & 7,8 & 0,45 & $*$ & $*$ & $*$ & 6,4 & 3,9 \\
\hline 125 & 8,4 & 0,76 & 40,7 & $*$ & * & 9,8 & * \\
\hline 126 & 7,4 & 0,57 & 17,9 & $*$ & $*$ & 41,4 & 13,4 \\
\hline 127 & 6,8 & 0,59 & 13,5 & $*$ & $*$ & $*$ & $*$ \\
\hline 128 & 6,6 & 0,45 & 13,1 & $*$ & 27,6 & 20,8 & $*$ \\
\hline 129 & 7,1 & 0,59 & $*$ & $*$ & $*$ & 7,8 & 3,3 \\
\hline 130 & 8,0 & 0,59 & 14,0 & $*$ & 7,5 & 3,2 & 3,9 \\
\hline 131 & 6,5 & 0,55 & 26,0 & $*$ & $*$ & 2,2 & 4,7 \\
\hline 132 & 6,4 & 0,69 & $*$ & $*$ & $*$ & 13,2 & 4,2 \\
\hline 133 & 8,4 & 0,59 & $*$ & $*$ & $*$ & 7,1 & 2,8 \\
\hline 134 & 7,6 & 0,69 & 4,0 & $*$ & $*$ & 3,5 & 2,2 \\
\hline 135 & 6,0 & 0,53 & 6,1 & $*$ & $*$ & $*$ & $*$ \\
\hline 136 & 8,3 & 0,33 & $*$ & $*$ & $*$ & 1,6 & 3,6 \\
\hline 137 & 6,3 & 0,69 & 3,9 & $*$ & $*$ & 2,6 & \\
\hline 138 & 6,2 & 0,34 & $*$ & $*$ & $*$ & $*$ & 4,8 \\
\hline 139 & 6,9 & 0,59 & 6,1 & $*$ & 4,5 & $*$ & $*$ \\
\hline 140 & 6,6 & 0,60 & 1,7 & 1,6 & $*$ & $*$ & 1,0 \\
\hline 141 & 7,5 & 0,53 & 6,5 & $*$ & $*$ & $*$ & $*$ \\
\hline 142 & 8,4 & 0,63 & 45,7 & $*$ & 5,6 & 3,3 & 5,0 \\
\hline 143 & 7,9 & 0,61 & 11,5 & $*$ & * & $*$ & $*$ \\
\hline 144 & 6,7 & 0,48 & 16,7 & $*$ & $*$ & $*$ & 6,3 \\
\hline 145 & 6,0 & 0,51 & 3,6 & 2,6 & $*$ & 13,4 & 4,8 \\
\hline 146 & 7,9 & 0,73 & 9,3 & $*$ & 6,9 & 3,6 & 2,3 \\
\hline
\end{tabular}




\begin{tabular}{|c|c|c|c|c|c|c|c|}
\hline Proben-Nr. & $\begin{array}{c}\text { TS } \\
(\%)\end{array}$ & $\begin{array}{c}\mathrm{N} \\
(\%)\end{array}$ & $\begin{array}{c}\text { TC } \\
\left(\mathrm{mg} \mathrm{kg}^{-1}\right)\end{array}$ & $\begin{array}{c}\text { OTC } \\
\left(\mathrm{mg} \mathrm{kg}^{-1}\right)\end{array}$ & $\begin{array}{c}\mathrm{CTC} \\
\left(\mathrm{mg} \mathrm{kg}^{-1}\right)\end{array}$ & $\begin{array}{c}\text { SD } \\
\left(\mathrm{mg} \mathrm{kg}^{-1}\right)\end{array}$ & $\begin{array}{c}\mathrm{SM} \\
\left(\mathrm{mg} \mathrm{kg}^{-1}\right)\end{array}$ \\
\hline 147 & 7,4 & 0,72 & 9,0 & $*$ & $*$ & 4,4 & 3,4 \\
\hline 148 & 6,3 & 0,44 & 13,3 & * & * & * & 8,5 \\
\hline 149 & 6.7 & 0.58 & 7.6 & $*$ & $*$ & $*$ & $*$ \\
\hline 150 & 6,9 & 0,47 & 5,5 & * & * & 2,2 & * \\
\hline 151 & 8,3 & 0,67 & $*$ & * & 5,6 & 2,9 & 4,6 \\
\hline 152 & 7,6 & 0,86 & 6,7 & * & * & * & $*$ \\
\hline 153 & 6,6 & 0,35 & 14,4 & * & * & * & 5,0 \\
\hline 154 & 6,4 & 0,53 & 4,0 & * & * & 1,3 & 3,7 \\
\hline 155 & 6,5 & 0,70 & 2,4 & 136,2 & * & $*$ & 167,0 \\
\hline 156 & 6,6 & 0,80 & $*$ & $*$ & * & 2,8 & 3,8 \\
\hline 157 & 10,7 & 0,75 & $*$ & * & * & 3,8 & $*$ \\
\hline 158 & 9,1 & 1,01 & 11,8 & * & $*$ & 6,4 & $*$ \\
\hline 159 & 10,9 & 0,63 & 22,9 & * & * & 4,0 & 3,1 \\
\hline 160 & 12,4 & 0,90 & 32,8 & 5,0 & 15,4 & 6,2 & 3,1 \\
\hline 161 & 12,5 & 0,99 & 8,0 & 9,1 & 10,4 & 6,6 & 0,7 \\
\hline 162 & 10,1 & 0,78 & 5,5 & $*$ & $*$ & 6,2 & 18,5 \\
\hline 163 & 9,4 & 0,80 & $*$ & * & * & $*$ & 5,2 \\
\hline 164 & 9,3 & 0,83 & 12,1 & * & * & 5,6 & 3,7 \\
\hline 165 & 11,5 & 0,58 & $*$ & * & * & 2,9 & $*$ \\
\hline 166 & 11,0 & 0,58 & 37,4 & * & * & $*$ & 3,8 \\
\hline 167 & 10,0 & 0,82 & 7,7 & * & * & 8,2 & * \\
\hline 168 & 12,8 & 0,72 & $*$ & * & $*$ & $*$ & $*$ \\
\hline 169 & 14,6 & 1,01 & * & 8,1 & * & 20,0 & 2,5 \\
\hline 170 & 9,5 & 0,61 & $*$ & 1,8 & 2,0 & $*$ & 9,2 \\
\hline 171 & 10,1 & 0,79 & * & $*$ & $*$ & * & 5,6 \\
\hline 172 & 16,8 & 0,56 & $*$ & $*$ & $*$ & $*$ & $*$ \\
\hline 173 & 9,1 & 0,71 & 8,6 & * & $*$ & $*$ & $*$ \\
\hline 174 & 10,1 & 1,05 & $*$ & * & * & $*$ & $*$ \\
\hline 175 & 9,7 & 0,74 & 8,4 & $*$ & * & 3,2 & 2,1 \\
\hline 176 & 9,0 & 0,70 & 4,7 & * & * & 4,3 & 4,3 \\
\hline
\end{tabular}

* nicht nachweisbar; unterhalb der Bestimmungsgrenzen von $0,8 \mathrm{mg} \mathrm{kg}^{-1}$ für TC, CTC, SD, SM und unterhalb $1,0 \mathrm{mg} \mathrm{kg}^{-1}$ für OTC

k.A. $=$ keine Angabe 
Tabelle 40: Vergleich der mittels HPLC gemessenen Tetrazyklin-Konzentrationen im Sickerwasser unter Nullerde/Torf des Gefäßversuchs (Starkregenereignis 48 Stunden nach Gülleausbringung) mit den Ergebnissen der Referenzmessung (siehe Kapitel 3.4.1)

\begin{tabular}{lcc}
\hline Probe & \multicolumn{2}{c}{ Tetrazyklinkonzentration $\left(\boldsymbol{\mu g ~ \mathbf { l } ^ { - \mathbf { 1 } } )}\right.$} \\
\cline { 2 - 3 } & HPLC-Messung & Referenzmessung \\
\hline Gefäß 3, Fraktion I & 3,3 & 6,4 \\
Gefäß 3, Fraktion II & 8,2 & 5,7 \\
Gefäß 3, Fraktion III & 1,6 & 3,7 \\
Gefäß 3, Fraktion IV & 2,5 & 2,6 \\
Gefäß 4, Fraktion I & 4,9 & 1,0 \\
Gefäß 4, Fraktion II & 3,3 & 3,4 \\
Gefäß 4, Fraktion III & 4,9 & 3,0 \\
Gefäß 4, Fraktion IV & 4,1 & 2,9 \\
\hline HPLC High performanc liquid chromatogaphy &
\end{tabular}

HPLC High performance liquid chromatography 
Tabelle 41: Tetrazyklin-Gehalte im Boden der Kick-Brauckmann-Gefäße, Starkregenereignis 48 Stunden bzw. zehn Wochen nach Gülleausbringung

\begin{tabular}{|c|c|c|}
\hline \multirow[t]{2}{*}{ Probe } & \multicolumn{2}{|c|}{ Tetrazyklinkonzentration $\left(\mu \mathrm{g} \mathrm{kg}^{-1}\right)$} \\
\hline & 0- $12 \mathrm{~cm}$ Tiefe & $12-24 \mathrm{~cm}$ Tiefe \\
\hline \multicolumn{3}{|l|}{ Kontrollen } \\
\hline hS, Gefäß 5 & 9,3 & - \\
\hline 1U, Gefäß 9 & - & - \\
\hline T, Gefäß 5 & - & - \\
\hline \multicolumn{3}{|c|}{ Probennahme nach Beregnung 48 Stunden nach Gülleausbringung } \\
\hline hS, Gefäß 2 & 2190,5 & 1338,4 \\
\hline hS, Gefäß 3 & 4745,4 & 238,4 \\
\hline hS, Gefäß 4 & 1757,2 & 286,4 \\
\hline 1U, Gefäß 2 & 213,6 & 45,6 \\
\hline $1 \mathrm{U}$, Gefäß 3 & 8375,4 & 2676,9 \\
\hline 1U, Gefäß 4 & 9171,7 & 332,4 \\
\hline T, Gefäß 2 & 319,8 & 209,6 \\
\hline T, Gefäß 3 & 203,1 & 193,4 \\
\hline T, Gefäß 4 & 339,1 & 77,5 \\
\hline \multicolumn{3}{|c|}{ Probennahme nach Beregnung 10 Wochen nach Gülleausbringung } \\
\hline hS, Gefäß 10 & 723,8 & $<1$ \\
\hline hS, Gefäß 11 & 419,4 & 26,2 \\
\hline hS, Gefäß 12 & 515,0 & 49,9 \\
\hline 1U, Gefäß 10 & 17,0 & 7,9 \\
\hline 1U, Gefäß 11 & 27,7 & 2,9 \\
\hline 1U, Gefäß 12 & 339,7 & 209,6 \\
\hline T, Gefäß 10 & 207,8 & 54,8 \\
\hline T, Gefäß 11 & 217,0 & 80,9 \\
\hline T, Gefäß 12 & 95,7 & $<1$ \\
\hline $\begin{array}{ll}\text { hS } & \text { lehmiger Sand } \\
\text { IU } & \text { lehmiger Schluff } \\
\text { T } & \text { Nullerde/ Torf }\end{array}$ & & \\
\hline
\end{tabular}


Tabelle 42: Sulfadiazin-Gehalte im Boden der Kick-Brauckmann-Gefäße, Starkregenereignis 48 Stunden bzw. 10 Wochen nach Gülleausbringung

\begin{tabular}{lr}
\hline Probe & Sulfadiazinkonzentration $\left(\mu \mathbf{~ k g ~}^{-1}\right)$ \\
\cline { 2 - 2 } & 0- 12 cm Tiefe \\
\hline Kontrollen & $<1$ \\
\hline hS, Gefäß 5 & $<1$ \\
1U, Gefäß 9 & $<1$ \\
T, Gefäß 5 & $<$ \\
\hline
\end{tabular}

Probennahme nach Beregnung 48 Stunden nach Gülleausbringung

\begin{tabular}{lr}
\hline hS, Gefäß 6 & 141,6 \\
hS, Gefäß 7 & 153,3 \\
hS, Gefäß 8 & 331,4 \\
IU, Gefäß 6 & 77,5 \\
IU, Gefäß 7 & 60,1 \\
lU, Gefäß 8 & 61,2 \\
T, Gefäß 6 & 17,6 \\
T, Gefäß 7 & 40,7 \\
T, Gefäß 8 & 26,2 \\
\hline Probennahme nach Beregnung 10 Wochen nach Gülleausbringung \\
\hline hS, Gefäß 14 & 44,3 \\
hS, Gefäß 15 & 19,8 \\
hS, Gefäß 16 & 32,4 \\
IU, Gefäß 14 & 13,1 \\
lU, Gefäß 15 & 4,5 \\
lU, Gefäß 16 & 20,9 \\
T, Gefäß 14 & 32,9 \\
T, Gefäß 15 & 15,6 \\
T, Gefäß 16 & 16,3 \\
\hline hS lehmiger Sand & \\
IU lehmiger Schluff & \\
T Nullerde/ Torf & \\
\hline
\end{tabular}


Tabelle 43: Vergleich der mittels HPLC gemessenen Tetrazyklin-Konzentrationen im Sickerwasser des Lysimeterversuches mit den Werten der Referenzmessung (siehe Kapitel 3.4.2)

\begin{tabular}{lcc}
\hline Probe & \multicolumn{2}{c}{ Tetrazyklinkonzentration $\left(\boldsymbol{\mu g} \mathbf{~ l}^{\mathbf{- 1}}\right)$} \\
\cline { 2 - 3 } & HPLC-Messung & Referenzmessung \\
\hline Lys. 2, 19. KW & 1,3 & 0,1 \\
Lys. 13, 22. KW & 3,1 & 1,8 \\
Lys. 58, 21. KW & 0,9 & 1,8 \\
Lys. 65, 21. KW & 1,2 & 0,5 \\
\hline Lys. & Lysimeter & \\
KW & Kalenderwoche & \\
HPLC & High performance liquid chromatography &
\end{tabular}

Tabelle 44: Verlauf der Tetrazyklin-Gehalte (TC) im Boden ausgewählter Lysimeter während der Versuchsdauer (TC-Gabe über Gülle jeweils im Mai 2001 (Roggen früher, Mais später) und Mai 2002; Dosierung je Lysimeter: Lys. 4 und $28=45,0 \mathrm{mg}$ bzw. 44,3 mg TC; Lys. 13, 15, 33, $35=67,2$ bzw. 66,5 mg TC)

\begin{tabular}{lrrrrr}
\hline \multirow{2}{*}{ Probe } & \multicolumn{5}{c}{ Tetrazyklinkonzentration $\left(\boldsymbol{\mu g} \mathbf{~ k g}^{-\mathbf{1}}\right)$} \\
\cline { 2 - 6 } & Mai 2001 & Okt. 2001 & März 2002 & Mai 2002 & Okt. 2002 \\
\hline Lys 1 (KT) & $<1$ & 2,1 & 11,8 & $<1$ & $<1$ \\
Lys 4 & $<1^{*}$ & 20,5 & 22,5 & 54,0 & 67,6 \\
Lys 13 & $<1^{*}$ & 10,6 & $<1$ & 122,5 & 46,2 \\
Lys 15 & $67,1^{* *}$ & 25,4 & 17,4 & 63,9 & 85,5 \\
Lys 25 (KT) & $<1$ & 1,8 & 14,1 & $<1$ & $<1$ \\
Lys 28 & $<1^{*}$ & 5,1 & 16,5 & 71,6 & 31,3 \\
Lys 33 & $59,4^{* *}$ & 28,1 & 18,8 & 96,0 & 73,0 \\
Lys 35 & $<1^{*}$ & 12,8 & 12,4 & 60,4 & 72,7 \\
\hline$*$ & Gülle- bzw. TC-Gabe noch nicht erfolgt (Mais) & & & \\
$* *$ & Gülle- bzw. TC-Gabe erfolgt (Roggen) & & & \\
KT & Kontrolle &
\end{tabular}


Tabelle 45: Verlauf der Sulfadiazin-Gehalte (SD) im Boden ausgewählter Lysimeter während der Versuchsdauer (SD-Gabe über Gülle im Mai 2002; Dosierung je Lysimeter: Lys. 4 und $28=44,3 \mathrm{mg} \mathrm{SD}$; Lys. 13, 15, 33, $35=66,5 \mathrm{mg} \mathrm{SD}$ )

\begin{tabular}{lrrr}
\hline Probe & \multicolumn{3}{c}{ Sulfadiazinkonzentration $\left(\boldsymbol{\mu g} \mathbf{~ k g}^{-\mathbf{1}}\right)$} \\
\cline { 2 - 4 } & Mai $\mathbf{2 0 0 1}$ & Mai 2002 & Oktober 2002 \\
\hline Lys 1 (KT) & $<1$ & $<1$ & $<1$ \\
Lys 4 & $<1$ & 7,8 & 2,9 \\
Lys 13 & $<1$ & 15,5 & 1,7 \\
Lys 15 & $<1$ & 10,9 & 2,1 \\
Lys 25 (K) & $<1$ & $<1$ & $<1$ \\
Lys 28 & $<1$ & 9,6 & 1,5 \\
Lys 33 & $<1$ & 8,6 & 4,0 \\
Lys 35 & $<1$ & 9,1 & 3,0 \\
\hline KT Kontrolle & & &
\end{tabular}

Tabelle 46: Verlauf der Chlortetrazyklin-Gehalte im Boden ausgewählter Lysimeter während der Versuchsdauer (Nebenbefund)

\begin{tabular}{lrrrrr}
\hline Probe & \multicolumn{5}{c}{ Chlortetrazyklinkonzentration $\left(\boldsymbol{\mu g} \mathbf{~ k g}^{-\mathbf{1}}\right)$} \\
\cline { 2 - 5 } & Mai 2001 & Okt. 2001 & März 2002 & Mai 2002 & Okt. 2002 \\
\hline Lys 1 (KT) & $<2$ & $<2$ & $<2$ & $<2$ & $<2$ \\
Lys 4 & $2,6^{*}$ & 6,4 & 4,6 & 12,4 & 22,5 \\
Lys 13 & $12,6^{*}$ & 5,0 & $<2$ & 25,9 & 18,4 \\
Lys 15 & $7,2^{* *}$ & 4,3 & 3,7 & 9,6 & 22,6 \\
Lys 25 (KT) & $<2$ & $<2$ & 3,9 & $<2$ & $<2$ \\
Lys 28 & $10,0^{*}$ & 5,7 & 6,4 & 15,3 & 17,7 \\
Lys 33 & $10,6^{* *}$ & 4,9 & 4,6 & 21,8 & 21,3 \\
Lys 35 & $8,6^{*}$ & 4,0 & 4,0 & 8,0 & 24,8 \\
\hline$*$ & Gülle- bzw. TC-Gabe noch nicht erfolgt (Mais) & & & \\
$* *$ & Gülle- bzw. TC-Gabe erfolgt (Roggen) & & & \\
KT & Kontrolle & &
\end{tabular}




\section{Danksagung}

Für die exzellente Betreuung in Form von vielen hilfreichen Diskussionen und Ratschlägen und für die Übernahme des Korreferates möchte ich ganz herzlich Prof. Dr. C. Winckler von der Universität für Bodenkultur in Wien, Österreich, danken. Ebenfalls gilt mein herzlicher Dank für das in mich gesetzte Vertrauen dem ersten Referenten dieser Arbeit, Prof. Dr. Ir. H. Van den Weghe.

Dem Laborteam des FOSVWE in Vechta, namentlich Uwe Vehlow, Andre Peukert, Ingeborg Zumbrägel, Ruth Wigger und vor allem auch Martin Körner danke ich für die wertvolle Unterstützung in analytischen Fragen.

Für die Kreativität und Hilfsbereitschaft in der Entwicklung und Umsetzung von Versuchsanstellungen möchte ich dem Werkstattteam des FOSVWE, namentlich Thomas Kruthoff, Hartmut Liebenow und Heinz Siebenand, und den Versuchstechnikern Barbara Thomann, Manfred Kayser und Hans-Jürgen Technow danken. Letzterem vor allem explizit für die aufopferungsvolle Tierbetreuung.

Viktoria List hat sich als studentische Hilfskraft bei der Durchführung vieler Arbeiten, vor allem im Labor, unentbehrlich gemacht- vielen Dank, Viktoria.

Nicht zuletzt gilt mein Dank für die gute Zusammenarbeit auch der Landwirtschaftskammer Weser-Ems, namentlich Dr. G. Steffens, Herr van Loo und Iris Höft und der Zentrumsabteilung für Lebensmitteltechnologie und -toxikologie der Tierärztlichen Hochschule Hannover, namentlich Dr. G. Hamscher.

An Heike Nordhoff-Felis und Uwe Vehlow ein herzlicher Dank für das Korrekturlesen der Arbeit.

Und schließlich gilt mein ganz besonderer Dank meinem Mann Fritjof, meiner Familie und meinen Freunden für all die Unterstützung und das Verständnis, das sie mir in der nicht immer leichten Zeit des Entstehens dieser Arbeit entgegengebracht haben. 


\section{Lebenslauf}

Name

geboren am:

Schule:

Studium:

Tätigkeiten:
Heike Engels

03.12.1973 in Bremen

1980-1984 Grundschule Uphusen

1984-1986 Orientierungsstufe Achim

1986-1993 Gymnasium Achim, Abschluss: Abitur

1993-1998 Agrarökologie an Universität Rostock,

Abschluss: Diplom

1999-2000 Akademisches Auslandsamt der Universität Rostock, wissenschaftliche Mitarbeit

2001-2003 Forschungs- und Studienzentrum für Veredelungswirtschaft Weser-Ems der Georg-August-Universität Göttingen, wissenschaftliche Mitarbeit 